\title{
A STUDY OF SINGULARITIES ON RATIONAL CURVES VIA SYZYGIES
}

\author{
David Cox, Andrew R. Kustin ${ }^{1}$, Claudia Polini ${ }^{2}$, And Bernd Ulrich ${ }^{3}$
}

\begin{abstract}
Consider a rational projective curve $\mathcal{C}$ of degree $d$ over an algebraically closed field $\boldsymbol{k}$. There are $n$ homogeneous forms $g_{1}, \ldots, g_{n}$ of degree $d$ in $B=k[x, y]$ which parameterize $\mathcal{C}$ in a birational, base point free, manner. We study the singularities of $\mathcal{C}$ by studying a Hilbert-Burch matrix $\varphi$ for the row vector $\left[g_{1}, \ldots, g_{n}\right]$. In the "General Lemma" we use the generalized row ideals of $\varphi$ to identify the singular points on $\mathcal{C}$, their multiplicities, the number of branches at each singular point, and the multiplicity of each branch.

Let $p$ be a singular point on the parameterized planar curve $\mathcal{C}$ which corresponds to a generalized zero of $\varphi$. In the "Triple Lemma" we give a matrix $\varphi^{\prime}$ whose maximal minors parameterize the closure, in $\mathbb{P}^{2}$, of the blow-up at $p$ of $\mathcal{C}$ in a neighborhood of $p$. We apply the General Lemma to $\varphi^{\prime}$ in order to learn about the singularities of $\mathcal{C}$ in the first neighborhood of $p$. If $\mathcal{C}$ has even degree $d=2 c$ and the multiplicity of $\mathcal{C}$ at $p$ is equal to $c$, then we apply the Triple Lemma again to learn about the singularities of $\mathcal{C}$ in the second neighborhood of $p$.

Consider rational plane curves $\mathcal{C}$ of even degree $d=2 c$. We classify curves according to the configuration of multiplicity $c$ singularities on or infinitely near $\mathcal{C}$. There are 7 possible configurations of such singularities. We classify the Hilbert-Burch matrix which corresponds to each configuration. The study of multiplicity $c$ singularities on, or infinitely near, a fixed rational plane curve $\mathcal{C}$ of degree $2 c$ is equivalent to the study of the scheme of generalized zeros of the fixed balanced Hilbert-Burch matrix $\varphi$ for a parameterization of $\mathcal{C}$. Let

$$
\mathrm{BalH}_{d}=\left\{\begin{array}{l|l}
\varphi & \begin{array}{l}
\varphi \text { is a } 3 \times 2 \text { matrix; each entry in } \varphi \text { is a homogeneous } \\
\text { form of degree } c \text { from } B ; \text { and } \operatorname{ht} I_{2}(\varphi)=2
\end{array}
\end{array}\right\}
$$

2010 Mathematics Subject Classification. 14H20, 13H15, 13H10, 13A30, 14H50, 14H10, 14Q05, $65 \mathrm{D} 17$.

Key words and phrases. Axial singularities, Balanced Hilbert-Burch matrix, Base point free locus, Birational locus, Birational parameterizations, Branches of a rational plane curve, Conductor, Configuration of singularities, Generalized row ideal, Generalized zero of a matrix, Generic Hilbert-Burch matrix, Hilbert-Burch matrix, Infinitely near singularities, Jacobian matrix, Module of Kähler differentials, Multiplicity, Parameterization, Parameterization of a blow-up, Ramification locus, Rational plane curve, Rational plane quartics, Rational plane sextics, Scheme of generalized zeros, Singularities of multiplicity equal to degree divided by two, Strata of rational plane curves, Taylor resultant, Universal projective resolution, Veronese subring.

${ }^{1}$ Supported in part by the National Security Agency.

${ }^{2}$ Supported in part by the National Science Foundation and the National Security Agency.

${ }^{3}$ Supported in part by the National Science Foundation.
\end{abstract}


The group $G=\mathrm{GL}_{3}(\boldsymbol{k}) \times \mathrm{GL}_{2}(\boldsymbol{k})$ acts on $\mathrm{BalH}_{d}$ by way of $(\chi, \xi) \cdot \varphi=\chi \varphi \xi^{-1}$. We decompose $\mathrm{BalH}_{d}$ into a disjoint union of 11 orbits. Each orbit has the form $G \cdot M$, where $M$ is the closed irreducible subspace of affine space defined by the maximal order minors of a generic matrix.

We introduce the parameter space $\mathbb{A}_{d}$. Each element $\boldsymbol{g}$ of $\mathbb{A}_{d}$ is an ordered triple of $d$-forms from $B$, and each $\boldsymbol{g} \in \mathbb{A}_{d}$ induces a rational map $\Psi_{\boldsymbol{g}}: \mathbb{P}^{1}--\rightarrow \mathbb{P}^{2}$. We define

$$
\begin{aligned}
& \mathbb{T}_{d}=\left\{\boldsymbol{g} \in \mathbb{A}_{d} \mid \Psi_{\boldsymbol{g}} \text { is birational onto its image without base points }\right\} \text { and } \\
& \mathbb{B}_{d}=\left\{\begin{array}{l|l}
\boldsymbol{g} \in \mathbb{T}_{d} & \begin{array}{l}
\text { every entry in the corresponding homogeneous Hilbert-Burch } \\
\text { matrix has degree } d / 2
\end{array}
\end{array}\right\} .
\end{aligned}
$$

In practice we are only interested in the subset $\mathbb{T}_{d}$ of $\mathbb{A}_{d}$. Every element of $\mathbb{A}_{d}$ which is not in $\mathbb{T}_{d}$ corresponds to an unsuitable parameterization of a curve. We prove that if there is a multiplicity $c$ singularity on or infinitely near a point $p$ on a curve $\mathcal{C}$ of degree $d=2 c$, then the parameterization of $\mathcal{C}$ is an element of $\mathbb{B}_{d}$. We prove that $\mathbb{B}_{d} \subseteq \mathbb{T}_{d}$ are open subsets of $\mathbb{A}_{d}$. We identify an open cover $\cup \mathbb{B}_{d}^{(i)}$ of $\mathbb{B}_{d}$ and for each $\mathbb{B}_{d}^{(i)}$ in this open cover, we identify a generic Hilbert-Burch matrix which specializes to give a Hilbert-Burch matrix for $\boldsymbol{g}$ for each $\boldsymbol{g} \in \mathbb{B}_{d}^{(i)}$. As an application of this result, we identify a universal projective resolution for the graded Betti numbers

$$
0 \rightarrow B(-3 c)^{2} \rightarrow B(-2 c)^{3} \rightarrow B
$$

We decompose the space $\mathbb{B}_{d}$ of balanced triples into strata. Each stratum consists of those triples $\boldsymbol{g}$ in $\mathbb{B}_{d}$ for which the corresponding curve $\mathcal{C}_{\boldsymbol{g}}$ exhibits one particular configuration of multiplicity $c$ singularities.

We use the Jacobian matrix associated to the parameterization to identify the non-smooth branches of the curve as well as the multiplicity of each branch. The starting point for this line of reasoning is the result that if $D$ is an algebra which is essentially of finite type over the ring $C$, then the ramification locus of $D$ over $C$ is equal to the support of the module of Kähler differentials $\Omega_{D / C}$. The General Lemma is a local result. Once one knows the singularities $\left\{p_{i}\right\}$ on a parameterized curve $\mathcal{C}$, then the General Lemma shows how to read the multiplicity and number of branches at each point $p_{i}$ from the Hilbert-Burch matrix of the parameterization. The result about the Jacobian matrix is a global result. It describes, in terms of the parameterization, all of the points $p$ on $\mathcal{C}$ and all of the branches of $\mathcal{C}$ at $p$ for which the multiplicity is at least two. In contrast to General Lemma one may apply the Jacobian matrix technique before one knows the singularities on $\mathcal{C}$.

We use conductor techniques to study the singularity degree $\delta$. Let $\boldsymbol{g}=\left(g_{1}, g_{2}, g_{3}\right)$ be an element of $\mathbb{T}_{d}$ and $\mathcal{C}_{\boldsymbol{g}}$ be the corresponding parameterized plane curve. We produce a polynomial $c_{\boldsymbol{g}}$ whose factorization into linear factors gives the value of the invariant $\delta$ at each singular point of $\mathcal{C}_{\boldsymbol{g}}$. The polynomial $c_{\boldsymbol{g}}$ is obtained in a polynomial manner from the coefficients of the entries of a Hilbert-Burch matrix for $\boldsymbol{g}$. We use these ideas to produce closed sets in $\mathbb{B}_{d}$ which separate various configurations of singularities. To create $c_{\boldsymbol{g}}$, we start with the coordinate $\operatorname{ring} A_{\boldsymbol{g}}=\boldsymbol{k}\left[g_{1}, g_{2}, g_{3}\right] \subseteq$ $B$. If $\boldsymbol{V}$ is the $d^{\text {th }}$ Veronese subring of $B$ and $\mathfrak{c}_{\boldsymbol{g}}$ is the conductor $A_{\boldsymbol{g}}: \boldsymbol{V}$, then $c_{\boldsymbol{g}}$ generates the saturation of the extension of $\mathfrak{c}_{\boldsymbol{g}}$ to $B$.

In the final section of the paper, we apply our results to rational plane quartics. We exhibit a stratification of $\mathbb{B}_{4}$ in which every curve associated to a given stratum has the same configuration of singularities and we compute the dimension of each stratum.

\section{TABle of Contents}

0. Introduction, terminology, and preliminary results. 
1. The General Lemma.

2. The Triple Lemma.

3. The BiProj Lemma.

4. Singularities of multiplicity equal to degree divided by two.

5. The space of true triples of forms of degree $d$ : the base point free locus, the birational locus, and the generic Hilbert-Burch matrix.

6. Decomposition of the space of true triples.

7. The Jacobian matrix and the ramification locus.

8. The conductor and the branches of a rational plane curve.

9. Rational plane quartics: a stratification and the correspondence between the Hilbert-Burch matrices and the configuration of singularities.

Section 0. Introduction, terminology, AND PRELIMINARY RESUlts.

\section{Subsection 0.A Introduction.}

Consider a rational projective curve $\mathcal{C}$ of degree $d$ over an algebraically closed field $k$. This curve, by definition, is the closure of the image of a rational map $\Psi: \mathbb{P}^{1}--\rightarrow \mathbb{P}^{n-1}$. One can arrange the parameterization so that $\Psi$ has no base points and is birational onto its image. Thus, there are $n$ homogeneous forms $g_{1}, \ldots, g_{n}$ of degree $d$ in $B=\boldsymbol{k}[x, y]$ so that $\Psi(q)=\left[g_{1}(q): \cdots: g_{n}(q)\right]$ for all $q \in \mathbb{P}^{1}$ and

$$
\mathcal{C}=\left\{\Psi(q) \mid q \in \mathbb{P}^{1}\right\}
$$

One traditional approach to the study of $\mathcal{C}$ involves finding the polynomial relations $F \in \boldsymbol{k}\left[T_{1}, \ldots, T_{n}\right]$ on $g_{1}, \ldots, g_{n}$, with $F\left(g_{1}, \ldots, g_{n}\right)=0$. If one follows this line of investigation, then the defining equations of $\mathcal{C}$ form the ideal

$$
I(\mathcal{C})=\left\{F \in \boldsymbol{k}\left[T_{1}, \ldots, T_{n}\right] \mid F\left(g_{1}, \ldots, g_{n}\right)=0\right\}
$$

Our approach instead is to look at the linear relations on $g_{1}, \ldots, g_{n}$ with coefficients in $B$. We study the syzygy module

$$
\left\{\left[\begin{array}{c}
c_{1} \\
\vdots \\
c_{n}
\end{array}\right] \in B^{n} \mid \sum_{i=1}^{c} g_{i} c_{i}=0\right\}
$$

In particular, we focus on a Hilbert-Burch matrix $\varphi$ so that

$$
0 \rightarrow \bigoplus_{i=1}^{n-1} B\left(-d-d_{i}\right) \stackrel{\varphi}{\longrightarrow} B(-d)^{n} \stackrel{\left[g_{1}, \ldots, g_{n}\right]}{\longrightarrow} B
$$


is an exact sequence. It is plausible to learn about $\mathcal{C}$ from $\varphi$ because, for example, when $\mathcal{C}$ is a plane curve (that is, $n=3$ ), then the ideal $I(\mathcal{C})$ is generated by the resultant of the two polynomials $\sum_{i=1}^{3} T_{i} \varphi_{i, j}$, where $\varphi=\left(\varphi_{i, j}\right)$ and the two listed polynomials $(1 \leq j \leq 2)$ are viewed as homogeneous polynomials of degree $d_{1}$ and $d_{2}$ in the variables $x$ and $y$ with coefficients being linear forms in $k\left[T_{1}, T_{2}, T_{3}\right]$.

Section 1 is concerned with the General Lemma. We use the generalized row ideals of $\varphi$ to identify the singular points on $\mathcal{C}$, their multiplicities, and the number of branches at each singular point. Indeed, the parameterization $\Psi$ automatically parameterizes the branches of $\mathcal{C}$; see Observation 1.25. The General Lemma also uses the generalized row ideals of $\varphi$ to identify the multiplicity of each branch of $\mathcal{C}$.

Let $\mathcal{C}$ be a parameterized planar curve and let $d_{1} \leq d_{2}$, as shown in (0.1), with $n=3$, be the shifts for a homogeneous Hilbert-Burch matrix for a parameterization of $\mathcal{C}$. One consequence of the General Lemma is the observation that if $p$ is a singularity on $\mathcal{C}$, then the multiplicity of $p$ is either equal to $d_{2}$ or less than or equal to $d_{1}$; furthermore, there is a one-to-one correspondence between the generalized zeros of $\varphi$ and the singularities on $\mathcal{C}$ of multiplicity $d_{1}$ and $d_{2}$. (This observation was already known by the Geometric Modeling Community [30, 10], where these singularities are called axial singularities. Our contribution in this context is a complete description of all singularities on $\mathcal{C}$ (not just axial singularities) in terms of information which may be read from $\varphi$. We study space curves as well as planar curves and we calculate the information about the branches and the multiplicity.)

Let $p$ be a singular point on the parameterized planar curve $\mathcal{C}$ which corresponds to a generalized zero of $\varphi$. The Triple Lemma in Section 2 concerns the blow-up $\mathcal{C}^{\prime}$ of $\mathcal{C}$ centered at $p$. Theorem 2.1 gives a matrix $\varphi^{\prime}$ whose maximal minors parameterize the closure, in $\mathbb{P}^{2}$, of the blow-up at $p$ of $\mathcal{C}$ in a neighborhood of $p$. We are able to apply the General Lemma to $\varphi^{\prime}$ in order to learn about the singularities of $\mathcal{C}$ in the first neighborhood of $p$. If $\mathcal{C}$ has even degree $d=2 c$ and the multiplicity of $\mathcal{C}$ at $p$ is equal to $c$, then we are able to apply Theorem 2.1 again and learn about the singularities of $\mathcal{C}$ in the second neighborhood (that is, after two blow-ups) of $p$. In section 2 we also prove that if $p$ is a point on $\mathcal{C}$ and $q$ is a singular point of multiplicity $c$ infinitely near $p$, then the multiplicity of $p$ is also equal to $c$. It follows that if there is a multiplicity $c$ singularity on or infinitely near $\mathcal{C}$, then every entry in a homogeneous Hilbert-Burch matrix for a parameterization of $\mathcal{C}$ is a homogeneous form of degree $c$.

The rest of the paper, with the exception of Section 7, is about rational plane curves of even degree $d=2 c$. We classify curves according to the configuration of multiplicity $c$ singularities on or infinitely near $\mathcal{C}$. There are 7 possible configurations of such singularities. In Section 4, we classify the Hilbert-Burch matrix which corresponds to each configuration. For example, one assertion of Theorem 4.8 is as follows. If $\mathcal{C}$ is a rational plane curve of degree $d=2 c$ and there is a singularity on 
$\mathcal{C}$ of multiplicity $c$ such that after one blow-up the singularity still has multiplicity $c$ and after a second blow-up the singularity still has multiplicity $c$, then there exists a linear automorphism $\Lambda$ of $\mathbb{P}^{2}$ and linearly independent forms $Q_{1}, Q_{2}, Q_{3}$ in $B_{c}$ such that $\Lambda \mathcal{C}$ is parameterized by the maximal order minors of

$$
\left[\begin{array}{cc}
Q_{1} & Q_{2} \\
Q_{3} & Q_{1} \\
0 & Q_{3}
\end{array}\right]
$$

The multiplicity $c$ singularity on $\Lambda \mathcal{C}$ occurs at the point $[0: 0: 1]$. The repeated entry $Q_{3}$ ensures that there is a multiplicity $c$ singularity in the first neighborhood of $p$. The repeated entry $Q_{1}$ ensures that there is a multiplicity $c$ entry in the second neighborhood of $c$.

Section three addresses the following question. If one has an arbitrary HilbertBurch matrix (or, equivalently, an arbitrary parameterization of a curve), how can one determine what the Hilbert-Burch matrix will be once enough row and column operations have been applied to transform it into the pretty form which is promised by Theorem 4.8? The other motivation for Section 3 is our desire to separate the set of parameterizations into strata where each parameterization in a given stratum gives rise to a curve with a predetermined configuration of singularities, and the closure of any given stratum is the union of all strata less than or equal to the given stratum. This stratification is carried out in Section 6. The results of Section 3 are used to determine the appropriate stratum for each parameterization.

At any rate, the study of multiplicity $c$ singularities on, or infinitely near, a fixed rational plane curve $\mathcal{C}$ of degree $2 c$ is equivalent to the study of the scheme of generalized zeros of the fixed balanced Hilbert-Burch matrix $\varphi$ for a parameterization of $\mathcal{C}$. We prove that the projection maps

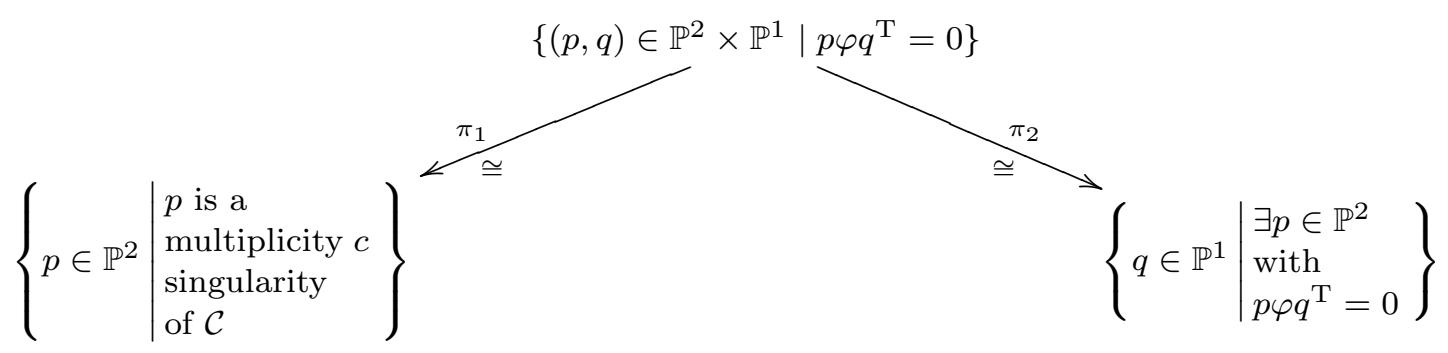

are isomorphisms and we identify the defining equations for each of the three schemes. The scheme on the lower left is the scheme of interest. The scheme on the lower right does not appear to have any intrinsic significance; however, it is a subscheme of $\mathbb{P}^{1}$, so it is easy to make computations concerning this scheme. In particular, our ultimate criteria in Theorem 3.22 for determining the configuration 
of multiplicity $c$ singularities on or infinitely near $\mathcal{C}$ amount to looking at the factorization of the greatest common divisor of the $3 \times 3$ minors of a matrix $A$ whose entries are linear forms in $k\left[u_{1}, u_{2}\right]$. The matrix $A$ is created quickly from $\varphi$ by way of extracting the variables $x$ and $y$ from the critical equation $\left[T_{1}, T_{2}, T_{3}\right] \varphi\left[\begin{array}{l}u_{1} \\ u_{2}\end{array}\right]=0$. We write $\left[T_{1}, T_{2}, T_{3}\right] \varphi=\left[y^{c}, \ldots, x^{c}\right] C$, where $C$ is a matrix of linear forms in $k\left[T_{1}, T_{2}, T_{3}\right]$ and we write $C\left[\begin{array}{l}u_{1} \\ u_{2}\end{array}\right]=A\left[\begin{array}{l}T_{1} \\ T_{2} \\ T_{3}\end{array}\right]$. The ideal generated by the entries of the product matrix on either side of the most recent equation defines the subscheme of $\mathbb{P}^{2} \times \mathbb{P}^{1}$ on the top of the above picture. Of course, Theorem 3.22 is phrased in terms of multiplicity and therefore we are able to apply the Generic Freeness Lemma, by way of Theorem 5.13, to families of parameterizations.

As previously mentioned, Section 4 contains a classification of Hilbert-Burch matrices and how these matrices correspond to a particular configuration of multiplicity $c$ singularities. This classification is the starting point for our study of families of curves. We use it to prove that our strata are irreducible and to calculate their dimensions. This classification is also an appropriate place to begin a study of the other singularities on, or infinitely near, the curves $\mathcal{C}$ those with multiplicity not equal to $c$. We carry out this study in the classical situation of quartics in Section 9. We have begun a study of sextics beginning from exactly this point.

It is possible that Theorem 4.9 could be of independent interest. There is no explicit mention of curves in the statement of this result. Let $d=2 c$ be an even integer and

$\mathrm{BalH}_{d}=\left\{\varphi \mid \varphi\right.$ is a $3 \times 2$ matrix with entries in $B_{c}$ such that ht $\left.I_{2}(\varphi)=2\right\}$, where, as always, $B=\boldsymbol{k}[x, y]$. The group $G=\mathrm{GL}_{3}(\boldsymbol{k}) \times \mathrm{GL}_{2}(\boldsymbol{k})$ acts on $\mathrm{BalH}_{d}$ by way of $(\chi, \xi) \cdot \varphi=\chi \varphi \xi^{-1}$. In Theorem 4.9 we decompose $\mathrm{BalH}_{d}$ into a disjoint union of 11 orbits. Each orbit has the form $G \cdot M$, where $M$ is the closed irreducible subspace of affine space defined by the maximal order minors of a generic matrix.

In Section 5 we introduce the parameter space $\mathbb{A}_{d}=B_{d} \times B_{d} \times B_{d}$. Each element $\boldsymbol{g}$ of $\mathbb{A}_{d}$ is an ordered triple of $d$-forms from $B=\boldsymbol{k}[x, y]$ (so, $\mathbb{A}_{d}$ is an affine space of dimension $3 d+3$ ), and each $\boldsymbol{g} \in \mathbb{A}_{d}$ induces a rational map $\Psi_{\boldsymbol{g}}: \mathbb{P}^{1}--\rightarrow \mathbb{P}^{2}$. We define

$$
\left.\begin{array}{l}
\mathbb{T}_{d}=\left\{\boldsymbol{g} \in \mathbb{A}_{d} \mid \Psi_{\boldsymbol{g}} \text { is birational onto its image without base points }\right\} \text { and } \\
\mathbb{B}_{d}=\left\{\boldsymbol{g} \in \mathbb{T}_{d} \mid \begin{array}{l}
\text { every entry in a homogeneous Hilbert-Burch } \\
\text { matrix for } \boldsymbol{d}_{1}(\boldsymbol{g}) \text { has degree } d / 2
\end{array}\right.
\end{array}\right\} .
$$

(If $\boldsymbol{g}$ is the ordered triple $\left(g_{1}, g_{2}, g_{3}\right)$ of $\mathbb{A}^{d}$, then $\boldsymbol{d}_{1}(\boldsymbol{g})$ is the row vector $\left[g_{1}, g_{2}, g_{3}\right]$.) We call $\mathbb{T}_{d}$ the space of true triples of forms of degree $d$ and $\mathbb{B}_{d}$ the space of balanced true triples of forms of degree $d$. 
In practice we are only interested in the subset $\mathbb{T}_{d}$ of $\mathbb{A}_{d}$. Every element of $\mathbb{A}_{d}$ which is not in $\mathbb{T}_{d}$ corresponds to an unsuitable parameterization of a curve. As we have already seen, the results of Section 2 show that if there is a multiplicity $c$ singularity on or infinitely near a point $p$ on a curve $\mathcal{C}$ of degree $d=2 c$, then the parameterization of $\mathcal{C}$ is an element of $\mathbb{B}_{d}$. In Section 5 we prove that $\mathbb{B}_{d} \subseteq \mathbb{T}_{d}$ are open subsets of $\mathbb{A}_{d}$. We also identify an open cover $\cup \mathbb{B}_{d}^{(i)}$ of $\mathbb{B}_{d}$ and for each $\mathbb{B}_{d}^{(i)}$ in this open cover, we identify a generic Hilbert-Burch matrix which specializes to give a Hilbert-Burch matrix for $\boldsymbol{g}$ for each $\boldsymbol{g} \in \mathbb{B}_{d}^{(i)}$.

Corollary 5.46 is another result which could be of independent interest. We identify a universal projective resolution $\mathbb{U} \mathbb{R}_{\mathbb{Z}}$ for the graded Betti numbers

$$
0 \rightarrow B(-3 c)^{2} \rightarrow B(-2 c)^{3} \rightarrow B .
$$

The resolution $\mathbb{U P R} \mathbb{R}_{\mathbb{Z}}$ is built over the $\operatorname{ring}\left(\boldsymbol{R}_{\mathbb{Z}}\right)_{w_{\mathbb{Z}}}[x, y]$, where $\boldsymbol{R}_{\mathbb{Z}}$ is a polynomial ring over $\mathbb{Z}, w_{\mathbb{Z}}$ is a non-zero homogeneous element in $\boldsymbol{R}_{\mathbb{Z}}$, and $\left(\boldsymbol{R}_{\mathbb{Z}}\right)_{w_{\mathbb{Z}}}$ is the localization of $\boldsymbol{R}_{\mathbb{Z}}$ at the multiplicatively closed set $\left\{1, w_{\mathbb{Z}}, w_{\mathbb{Z}}^{2}, \ldots\right\}$. If $(0.2)$ is a minimal homogeneous resolution of $B / I$ over $B=\boldsymbol{k}[x, y]$, then there exists a homomorphism $\left(\boldsymbol{R}_{\mathbb{Z}}\right)_{w_{\mathbb{Z}}} \rightarrow \boldsymbol{k}$ so that $\mathbb{U} \mathbb{R}_{\mathbb{Z}} \otimes_{\left(\boldsymbol{R}_{\mathbb{Z}}\right)_{w_{\mathbb{Z}}}} \boldsymbol{k}$ is a minimal homogeneous resolution of $B / I$ over $B$.

In Section 6 we decompose the space $\mathbb{B}_{d}$ of balanced triples into strata. Each stratum consists of those triples $\boldsymbol{g}$ in $\mathbb{B}_{d}$ for which the corresponding curve $\mathcal{C}_{\boldsymbol{g}}$ exhibits one particular configuration of multiplicity $c$ singularities. If $\mathcal{C}$ is a parameterized plane curve of degree $d=2 c$, then there are 7 possible configurations of multiplicity $c$ singularities on or infinitely near $\mathcal{C}$ :

$$
\mathrm{CP}=\{\emptyset,\{c\},\{c, c\},\{c, c, c\},\{c: c\},\{c: c, c\},\{c: c: c\}\},
$$

where a colon indicates an infinitely near singularity, a comma indicates a different singularity on the curve, and $\emptyset$ indicates that there are no singularities of multiplicity $c$ on, or infinitely near, $\mathcal{C}$. For example, if $\boldsymbol{g} \in S_{c: c: c}$, then there is exactly one multiplicity $c$ singularity $p$ on the corresponding curve $\mathcal{C}_{\boldsymbol{g}}$ and there are two multiplicity $c$ singularities infinitely near to $p$ : one in the first neighborhood of $p$ and one in the second neighborhood of $p$; and if $\boldsymbol{g} \in S_{c: c, c}$, then there are exactly two multiplicity $c$ singularities on $\mathcal{C}_{\boldsymbol{g}}$ and exactly one of these has a multiplicity $c$ singularity infinitely near it. We decompose $\mathbb{B}_{d}$ as

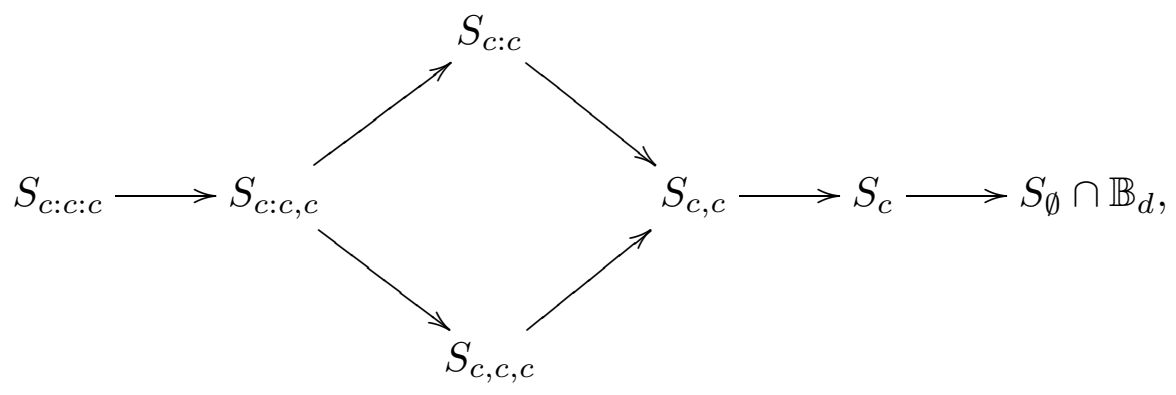


where $S_{\#^{\prime}} \rightarrow S_{\#}$ means that $S_{\#^{\prime}}$ is contained in the closure of $S_{\#}$. Think of CP as a poset with $\#^{\prime} \leq \#$ whenever we have drawn $S_{\#^{\prime}} \rightarrow S_{\#}$. We prove that each set $\mathbb{T}_{\#}=\cup_{\#^{\prime} \leq \#} S_{\#^{\prime}}$ is a closed irreducible subset of $\mathbb{B}_{d}$ and we compute the dimension of each stratum $S_{\#}$. The proof that $T_{\#}$ is closed uses Corollary 3.23 to associate numerical data to each $S_{\#}$, Theorem 5.13 to convert this numerical data into closed conditions on families of curves as calculated by way of the Hilbert-Burch matrix, and Theorem 5.30 to create a generic Hilbert-Burch matrix from the coefficients of the parameterization. The calculation of the dimension of $T_{\#}$ is based on the decomposition of balanced Hilbert-Burch matrices which takes place in Section 4.

In Section 7 we return to the hypotheses of Section 1 ; that is, $\mathcal{C}$ is a parameterized space curve of arbitrary degree and we learn about infinitely near singularities of arbitrary multiplicity. Remark 1.31 provides a method of parameterizing the branches of a parameterized curve. Theorem 7.2 shows that the Jacobian matrix associated to the parameterization identifies the non-smooth branches of the curve as well as the multiplicity of each branch. The starting point for this line of reasoning is the result that if $D$ is an algebra which is essentially of finite type over the ring $C$, then the ramification locus of $D$ over $C$ is equal to the support of the module of Kähler differentials $\Omega_{D / C}$. It is important to compare Theorems 1.8 and 7.4. Theorem 1.8 is a local result. Once one knows the singularities $\left\{p_{i}\right\}$ on a parameterized curve $\mathcal{C}$, then this result shows how to read $m_{\mathcal{C}, p_{i}}$ and $s_{\mathcal{C}, p_{i}}$, for each $p_{i}$, from the Hilbert-Burch matrix of the parameterization. Theorem 7.4 is a global result. It describes, in terms of the parameterization, all of the points $p$ on $\mathcal{C}$ and all of the branches of $\mathcal{C}$ at $p$ for which the multiplicity is at least two. In contrast to Theorem 1.8, one may apply Theorem 7.4 before one knows the singularities on $\mathcal{C}$.

Sections 1, 2, 3, and 7 are largely concerned with the multiplicity and the number of branches at each singularity on a curve. Section 8 deals with the singularity degree $\delta$. Let $\boldsymbol{g}=\left(g_{1}, g_{2}, g_{3}\right)$ be an element of $\mathbb{T}_{d}$ and $\mathcal{C}_{\boldsymbol{g}}$ be the corresponding parameterized plane curve. We produce a polynomial $c_{\boldsymbol{g}}$ whose factorization into linear factors gives the value of the invariant $\delta$ at each singular point of $\mathcal{C}_{\boldsymbol{g}}$. The polynomial $c_{\boldsymbol{g}}$ is obtained in a polynomial manner from the coefficients of the entries of a Hilbert-Burch matrix for $\boldsymbol{g}$. We use these ideas to produce closed sets in $\mathbb{B}_{d}$ which separate various configurations of singularities. To create $c_{\boldsymbol{g}}$ we start with the coordinate ring $A_{\boldsymbol{g}}=\boldsymbol{k}\left[g_{1}, g_{2}, g_{3}\right] \subseteq B=\boldsymbol{k}[x, y]$. If $\boldsymbol{V}$ is the $d^{\text {th }}$ Veronese subring of $B$ and $\mathfrak{c}_{\boldsymbol{g}}$ is the conductor $A_{\boldsymbol{g}}: \boldsymbol{V}$, then $c_{\boldsymbol{g}}$ generates the saturation of the extension of $\mathfrak{c}_{\boldsymbol{g}}$ to $B$.

In Section 9 we apply our results to rational plane quartics. It has been known for well over one hundred years (see, for example, Basset [2], Hilton [17], Namba [29], or Wall [33]) that there are 13 possible configurations of singularities on a rational plane quartic: 


$$
\begin{array}{llll}
(3: 1,1,1) & 2: 2: 2: 1,1 & (2: 2: 1,1),(2: 1,1) & (2: 1,1)^{3} \\
(3: 1,1) & 2: 2: 2: 1 & (2: 2: 1,1),(2: 1) & (2: 1,1)^{2},(2: 1) \\
3: 1 & & (2: 2: 1),(2: 1,1) & (2: 1,1),(2: 1)^{2} \\
& & (2: 1)^{3} & (2: 2: 1),(2: 1) .
\end{array}
$$

(The notation is fully defined around (0.5) and the full set of names is recorded in Section 9.) These 13 singularity configurations have been named and stratified many times already; see, for example, [3] and [34]. In Section 9, we show what our approach produces in this context. The techniques from Section 6 separate

$$
3: * \quad 2: 2: 2: * \quad(2: 2: *),(2: *), \quad \text { and } \quad(2: *)^{3} .
$$

The finer techniques of Section 7 separate the parameterizations according to the value of the invariant

$$
\sum_{p \in \operatorname{Sing} \mathcal{C}} m_{p}-s_{p},
$$

where $p$ roams over all of the singular points on $\mathcal{C}, m_{p}$ is the multiplicity of $\mathcal{C}$ at $p$ and $s_{p}$ is the number of branches of $\mathcal{C}$ at $p$. The conductor technique of Section 8 distinguishes the configuration of singularities $(2: 2: 1),(2: 1,1)$ from $(2: 2: 1,1),(2: 1)$. In Corollary 9.11 we exhibit a stratification of $\mathbb{B}_{4}$ in which every curve associated to a given stratum has the same configuration of singularities. We compute the dimension of each stratum.

We obtain a rational plane curve $d$ from each true triple of $d$-forms $g$ in $\mathbb{T}_{d}$. The space $\mathbb{T}_{d}$ is large and provides us with plenty of room for maneuvering. Nonetheless, it is important that we exhibit the precise relationship between the space $\mathbb{T}_{d}$ and the space $\mathbb{R} \mathbb{P} \mathbb{C}_{d}^{r}$ of right equivalence classes of Rational Plane Curves of degree $d$. Recall that the plane curves $\mathcal{C}$ and $\mathcal{C}^{\prime}$ are right equivalent if there exists a linear automorphism $\Lambda: \mathbb{P}^{2} \rightarrow \mathbb{P}^{2}$ with $\Lambda \mathcal{C}=\mathcal{C}^{\prime}$. We have surjective morphisms

$$
\mathbb{T}_{d} \stackrel{\alpha_{1}}{\longrightarrow} \mathbb{P T}_{d} \stackrel{\alpha_{2}}{\longrightarrow} \mathbb{R} \mathbb{P} \mathbb{C}_{d} \stackrel{\alpha_{3}}{\longrightarrow} \mathbb{R} \mathbb{P} \mathbb{C}_{d}^{r},
$$

where $\mathbb{P} \mathbb{T}_{d}$ is the space of degree $d$ base point free morphisms from $\mathbb{P}^{1}$ to $\mathbb{P}^{2}$ which are birational onto their image and $\mathbb{R} \mathbb{P} \mathbb{C}_{d}$ is the space of Rational Plane Curves of degree $d$. If $\Psi: \mathbb{P}^{1} \rightarrow \mathbb{P}^{2}$ is an element of $\mathbb{P T}_{d}$, then the fiber of $\alpha_{1}$ over $\Psi$ is isomorphic to $\mathrm{GL}_{1}(\boldsymbol{k})$. (Indeed, if $\boldsymbol{g}=\left(g_{1}, g_{2}, g_{3}\right)$ is in $\mathbb{T}_{d}$ and $u$ is a non-zero element of $\boldsymbol{k}$, then $\boldsymbol{g}$ and $u \boldsymbol{g}=\left(u g_{1}, u g_{2}, u g_{3}\right)$ give rise to the same morphism $\mathbb{P}^{1} \rightarrow \mathbb{P}^{2}$.) If $\mathcal{C}$ is an element of $\mathbb{R P} \mathbb{C}_{d}$, then the fiber of $\alpha_{2}$ over $\mathcal{C}$ is isomorphic to $\mathrm{SL}_{2}(\boldsymbol{k})$. Indeed, if $\Psi: \mathbb{P}^{1} \rightarrow \mathbb{P}^{2}$ is a morphism and $\xi: \mathbb{P}^{1} \rightarrow \mathbb{P}^{1}$ is a linear automorphism, then $\Psi$ 
and $\Psi \circ \xi$ give rise to same curve. The definition of right equivalence yields that the fiber of $\alpha_{3}$ over each equivalence class of $\mathbb{R P} \mathbb{C}_{d}^{r}$ is isomorphic to $\mathrm{SL}_{3}(\boldsymbol{k})$. All together the fiber of $\alpha_{3} \circ \alpha_{2} \circ \alpha_{1}$ over any equivalence class of $\mathbb{R P} \mathbb{C}_{d}^{r}$ is isomorphic to $\mathrm{GL}_{1}(\boldsymbol{k}) \times \mathrm{SL}_{2}(\boldsymbol{k}) \times \mathrm{SL}_{3}(\boldsymbol{k})$ and has dimension 12 .

\section{Subsection 0.B Terminology.}

All of our work takes place over a field $\boldsymbol{k}$. We write $\boldsymbol{k}^{*}$ for the multiplicative group $\boldsymbol{k} \backslash\{0\}$. Often (but not always) the field is algebraically closed. Sometimes the field $\boldsymbol{k}$ has characteristic zero. Projective space $\mathbb{P}^{n}$ means projective space $\mathbb{P}_{\boldsymbol{k}}^{n}$ over $\boldsymbol{k}$. Let $\mathcal{O}_{\mathcal{C}, p}$ represent the local ring of the curve $\mathcal{C}$ at the rational point $p$. The multiplicity of $\mathcal{C}$ at $p$, denoted $m_{p}$ (or $m_{\mathcal{C}, p}$ if there is any ambiguity about what $\mathcal{C}$ is), is the multiplicity of the local ring $\mathcal{O}_{\mathcal{C}, p}$. (Recall that the multiplicity of the $d$-dimensional Notherian local ring $(A, \mathfrak{m})$ is $e(A)=\lim _{n \rightarrow \infty} \frac{d ! \lambda_{A}\left(A / \mathfrak{m}^{n}\right)}{n^{d}}$, where $\lambda_{A}(M)$ is the length of the $A$-module $M$.) Let $\widehat{\mathcal{O}_{\mathcal{C}, p}}$ be the completion of $\mathcal{O}_{\mathcal{C}, p}$ with respect to its maximal ideal $\mathfrak{m}_{\mathcal{C}, p}$. Each minimal prime ideal of $\widehat{\mathcal{O}_{\mathcal{C}, p}}$ is called a branch of $\mathcal{C}$ at $p$. If $\mathcal{J}$ is a minimal prime of $\widehat{\mathcal{O}_{\mathcal{C}}, p}$, then the multiplicity of the local ring $\widehat{\mathcal{O}_{\mathcal{C}, p}} / \mathcal{J}$ is called the multiplicity of the branch $\mathcal{J}$. The number of branches of $\mathcal{C}$ at $p$ is denoted $s_{p}$ (or $\left.s_{\mathcal{C}, p}\right)$. The singularity degree of $\mathcal{C}$ at the point $p$, denoted $\delta_{p}$ (or $\delta_{\mathcal{C}, p}$ ), is $\lambda\left(\overline{\mathcal{O}_{\mathcal{C}, p}} / \mathcal{O}_{\mathcal{C}, p}\right)$, where $\overline{\mathcal{O}_{\mathcal{C}, p}}$ is the integral closure of $\mathcal{O}_{\mathcal{C}, p}$ and $\lambda$ means $\lambda_{\mathcal{O}_{\mathcal{C}, p}}$. The invariant $\delta_{p}$ may also be realized as $\delta_{p}=\sum_{q}\left(\begin{array}{c}m_{q} \\ 2\end{array}\right)$, where $q$ varies over $p$ together with "all singularities infinitely near to $p$ ". This notion is described below.

Let $p$ be a point on the curve $\mathcal{C}$ and let $\mathcal{C}^{\prime} \stackrel{\sigma}{\rightarrow} \mathcal{C}$ be the blow-up of $\mathcal{C}$ at $p$. The points in the first neighborhood of $p$ are the points on $\mathcal{C}^{\prime}$ which map to $p$. There are finitely many such points. The local rings of the points in the first neighborhood of $p$ are exactly the local rings of $\operatorname{Proj}(\mathcal{O}[\mathfrak{m} t])$, where $(\mathcal{O}, \mathfrak{m})=\left(\mathcal{O}_{\mathcal{C}, p}, \mathfrak{m}_{p}\right)$. As $\operatorname{dim} \mathcal{O}=1$, and $k$ is infinite, there exists $f \in \mathfrak{m}$ so that $\mathfrak{m}^{r+1}=f \mathfrak{m}^{r}$ for some $r \geq 0$; hence, $\operatorname{Proj}(\mathcal{O}[\mathfrak{m} t])=\operatorname{Spec}\left(\mathcal{O}\left[\frac{\mathfrak{m}}{f}\right]\right)$ and therefore the local rings of the points in the first neighborhood of $p$ are exactly the localizations of $\mathcal{O}\left[\frac{\mathfrak{m}}{f}\right]$ at one of its finitely many maximal ideals. Apply this process to each point in the first neighborhood of $p$ to obtain the points in the second neighborhood of $p$. Repeat the process until all of the points in the $r^{\text {th }}$ neighborhood of $p$ are smooth points, for some $r$. The union of the singular points in the $i^{\text {th }}$ neighborhood of $p$, for $1 \leq i \leq r$, is called the set of singularities of $\mathcal{C}$ infinitely near to $p$. All of the local rings for the singularities of $\mathcal{C}$ infinitely near to $p$ occur between $\mathcal{O}$ and its integral closure $\overline{\mathcal{O}}$, which is finitely generated as a $\mathcal{O}$-module.

Notice that we reserve the phrase "singularity of $\mathcal{C}$ " to refer to points on $\mathcal{C}$ which are singular points and we reserve the phrase "infinitely near singularity of $\mathcal{C}$ " to 
refer to singular points which are not on $\mathcal{C}$, but which appear after a finite sequence of blow-ups has occurred.

When we write that the multiplicity sequence for the oscnode $q_{0}$ on the curve $\mathcal{C}_{0}$ is $2: 2: 2: 1,1$, we mean that there is a sequence of blow-ups:

$$
\mathcal{C}_{0} \stackrel{\sigma_{1}}{\longleftarrow} \mathcal{C}_{1} \stackrel{\sigma_{2}}{\longleftarrow} \mathcal{C}_{2} \stackrel{\sigma_{3}}{\longleftarrow} \mathcal{C}_{3}
$$

and a sequence of points $q_{0}$ on $\mathcal{C}_{0}, q_{1}$ on $\mathcal{C}_{1}, q_{2}$ on $\mathcal{C}_{2}$, and $q_{3} \neq q_{3}^{\prime}$ on $\mathcal{C}_{3}$, such that $\sigma_{i}$ is the blowup of $\mathcal{C}_{i-1}$ centered at $q_{i-1}$ for $1 \leq i \leq 3$,

$$
\begin{gathered}
\sigma_{i}^{-1}\left(q_{i-1}\right)=\left\{\begin{array}{cl}
q_{i} & \text { if } 1 \leq i \leq 2 \\
\left\{q_{3}, q_{3}^{\prime}\right\} & \text { if } i=3,
\end{array}\right. \\
m_{\mathcal{C}_{0}, q_{0}}=m_{\mathcal{C}_{1}, q_{1}}=m_{\mathcal{C}_{2}, q_{2}}=2 \text { and } \quad m_{\mathcal{C}_{3}, q_{3}}=m_{\mathcal{C}_{3}, q_{3}^{\prime}}=1 .
\end{gathered}
$$

So, in particular,

$$
\delta_{q_{0}}=\sum_{i=0}^{2}\left(\begin{array}{c}
m_{q_{i}} \\
2
\end{array}\right)=3 \quad \text { and } \quad s_{q_{0}}=2
$$

because there are two smooth points on $\mathcal{C}_{3}$ which lie over $q_{0}$. When we write that the configuration of singularities of the curve $\mathcal{C}$ is described by $(2: 2: 1,1),(2: 1)$, we mean that there are exactly 2 singularities on $\mathcal{C}$ : one is a tacnode and the other is an ordinary cusp. There is exactly one singular point in the first neighborhood of the tacnode. This point has multiplicity 2 . There are exactly two points in the second neighborhood of the tacnode; both of these points are smooth points. There is exactly one point in the first neighborhood of the cusp and this point is a smooth point.

All rings in this paper are commutative and Noetherian. The grade of a proper ideal $I$ in a ring $R$ is the length of the longest regular sequence on $R$ in $I$. The expression "let $(A, \mathfrak{m}, \boldsymbol{k})$ be a local ring" means that $A$ is a local ring with unique maximal ideal $\mathfrak{m}$ and $k$ is the residue class field $A / \mathfrak{m}$. If $(A, \mathfrak{m})$ is a local ring and $z$ is an indeterminate over $A$, then

$$
A(z) \text { means the ring } A[z]_{\mathfrak{m} A[z]} \text {. }
$$

In other words, $A(z)$ is the localization of the polynomial ring $A[z]$ at the prime ideal $\mathfrak{m} A[z]$.

If $M$ is a matrix, then $I_{r}(M)$ is the ideal generated by the $r \times r$ minors of $M$ and $M^{\mathrm{T}}$ is the transpose of $M$. If $L$ is a graded module over a graded ring $B=B_{0} \oplus B_{1} \oplus \ldots$, then the $B_{0}$-module $L_{d}$ is the homogeneous component of $L$ of degree $d$. We write $\mu(L)$ to denote the minimal number of generators of the 
module $L$. This concept makes sense whenever $L$ is a finitely generated module over a local ring or $L$ is a finitely generated graded module over a graded ring with a unique maximal homogeneous ideal. If $\boldsymbol{T}=\left(T_{i, j}\right)$ is a matrix of indeterminates and $R$ is a ring, then $R[\boldsymbol{T}]$ is the polynomial $\operatorname{ring} R\left[\left\{T_{i, j}\right\}\right]$. If $I$ is an ideal of a ring $R$, then we write $\operatorname{dim} I$ to represent the Krull dimension of the quotient ring $R / I$. In particular, if $I$ is a homogeneous ideal in a graded ring $R$ with unique maximal homogeneous ideal $\mathfrak{m}$, then $I$ is a zero-dimensional ideal if and only if $I$ is an $\mathfrak{m}$-primary ideal.

If $v_{1}, \ldots, v_{r}$ are elements in a vector space $V$, then we write $\left\langle v_{1}, \ldots, v_{r}\right\rangle$ to mean the subspace of $V$ spanned by $v_{1}, \ldots, v_{r}$.

If $R$ is a ring, then we write $\operatorname{Quot}(R)$ for the total quotient ring of $R$; that is, $\operatorname{Quot}(R)=U^{-1} R$, where $U$ is the set of non zerodivisors on $R$. If $R$ is a domain, then the total quotient ring of $R$ is usually called the quotient field of $R$. The integral closure $\bar{R}$ of a ring $R$ is the integral closure of $R$ in the $\operatorname{Quot}(R)$.

If $g_{1}, \ldots, g_{n}$ are elements of $B=\boldsymbol{k}[x, y]$ which generate an ideal of height two, then the Hilbert-Burch Theorem asserts that the relations on the row vector $\left[g_{1}, \ldots, g_{n}\right]$ fit into an exact sequence

$$
0 \rightarrow B^{n-1} \stackrel{\varphi}{\rightarrow} B^{n} \stackrel{\left[g_{1}, \ldots, g_{n}\right]}{\longrightarrow} B
$$

and that there is a unit $u$ in $B$ so that $g_{i}$ is equal to $(-1)^{i+1} u$ times the determinant of $\varphi$ with row $i$ removed. We call $\varphi$ a Hilbert-Burch matrix for $\left[g_{1}, \ldots, g_{n}\right]$. If $I$ and $J$ are ideals of a ring $R$, then the saturation of $I$ with respect to $J$ is $I: J^{\infty}=\bigcup_{i=1}^{\infty}\left(I: J^{i}\right)$. We write gcd to mean greatest common divisor. If $I$ is a homogeneous ideal in $k[x, y]$, then we denote the gcd of a generating set of $I$ by $\operatorname{gcd} I$; notice that this polynomial generates the saturation $I:(x, y)^{\infty}$.

Remark 0.7. The concept of "generalized row ideals" (as well as other related concepts) appears widely in the literature; see, for example, $[15,13,14]$. Let $M$ be a matrix with entries in a $\boldsymbol{k}$-algebra, where $\boldsymbol{k}$ is a field. A generalized row of $M$ is the product $p M$, a generalized column of $M$ is the product $M q^{\mathrm{T}}$, and a generalized entry of $M$ is the product $p M q^{\mathrm{T}}$, where $p$ and $q$ are non-zero row vectors with entries from $\boldsymbol{k}$. A generalized zero of $M$ is a generalized entry of $M$ which is zero. If $p M$ is a generalized row of $M$, then the ideal $I_{1}(p M)$ is called a generalized row ideal of $M$. If $M q^{\mathrm{T}}$ is a generalized column of $M$, then the ideal $I_{1}\left(M q^{\mathrm{T}}\right)$ is called a generalized column ideal of $M$.

Remark 0.8. Often, we think of a point $p=\left[a_{1}: \cdots: a_{n}\right] \in \mathbb{P}^{n-1}$ as a row vector $\left[a_{1}, \ldots, a_{n}\right]$, where $\left[a_{1}, \ldots, a_{n}\right]$ is any one of the row vectors that represent the point $p$ in $\mathbb{P}^{n-1}$. For example, if $M$ is a matrix with $n$ rows, then the point $p$ in $\mathbb{P}^{n-1}$ 
gives rise to a unique generalized row ideal $I_{1}\left(\left[a_{1}, \ldots, a_{n}\right] M\right)$ of $M$ since the ideals $I_{1}\left(\left[a_{1}, \ldots, a_{n}\right] M\right)$ and $I_{1}\left(\left[\lambda a_{1}, \ldots, \lambda a_{n}\right] M\right)$ are equal for all non-zero constants $\lambda$ of $k$. It is convenient, and unambiguous, to write $I_{1}(p M)$, with $p \in \mathbb{P}^{n-1}$, instead of $I_{1}\left(\left[a_{1}, \ldots, a_{n}\right] M\right)$.

Remark 0.9. If $g_{1}, \ldots, g_{n}$ are linearly independent homogeneous polynomials of the same degree in $k[x, y]$, then we consider the rational map $\Psi: \mathbb{P}^{1}--\rightarrow \mathbb{P}^{n-1}$ which sends the point $q$ of $\mathbb{P}^{1} \backslash V\left(g_{1}, \ldots, g_{n}\right)$ to $\left[g_{1}(q): \cdots: g_{n}(q)\right] \in \mathbb{P}^{n-1}$. The image of $\Psi$ is a curve $\mathcal{C}$ in $\mathbb{P}^{n-1}$. Let $\varphi$ be a matrix of relations on the row vector $g=\left[g_{1}, \ldots, g_{n}\right]$. Row operations on $\varphi$ correspond to replacing $\varphi$ by $\varphi^{\prime}=\chi \varphi$, where $\chi$ is an invertible $n \times n$ matrix with entries in $k$. Now $\varphi^{\prime}$ is a matrix of relations on the row vector $g^{\prime}=g \chi^{-1}$. Let $\Lambda: \mathbb{P}^{n-1} \rightarrow \mathbb{P}^{n-1}$ be the linear automorphism sending $p$ to $p \chi^{-1}$. When we replace $g$ by $g^{\prime}$, the rational map $\Psi$ and the curve $\mathcal{C}$ become $\Lambda \circ \Psi$ and $\Lambda(\mathcal{C})$, respectively. Column operations on $\varphi$ have no effect on the row vector $g$, the rational map $\Psi$, or the curve $\mathcal{C}$.

\section{Subsection 0.C Preliminary Results.}

The following result is established in [25]; it interprets the birationality of a parameterization of a curve in terms of the generalized Hilbert-Burch matrix which corresponds to this parameterization. Recall that $R$ is called a standard graded algebra over the ring $R_{0}$ if $R$ is a graded ring $\bigoplus_{0<i} R_{i}$, with $R_{1}$ finitely generated as a module over $R_{0}$, and $R$ generated as an algebra over $R_{0}$ by $R_{1}$. If $R$ is a standard graded algebra over a field, then the multiplicity of $R$ is given by $e(R)=\lim _{n \rightarrow \infty} \frac{d ! \lambda_{R}\left(R / \mathfrak{m}^{n}\right)}{n^{d}}$, where $d$ is the Krull dimension of $R$ and $\mathfrak{m}$ is the maximal homogeneous ideal $\bigoplus_{1 \leq i} R_{i}$.

Theorem 0.10. Let $B$ be the standard graded polynomial ring $\boldsymbol{k}[x, y]$, with $\boldsymbol{k}$ an infinite field, $I$ be a height two ideal of $B$ generated by forms $g_{1}, \ldots, g_{n}$ of degree $d$, and $\varphi$ be a homogeneous Hilbert-Burch matrix for the row vector $\left[g_{1}, \ldots, g_{n}\right]$. If $C$ and $D$ are the standard graded $\boldsymbol{k}$-algebras $C=\boldsymbol{k}\left[I_{d}\right]$ and $D=\boldsymbol{k}\left[B_{d}\right], \boldsymbol{r}$ is the degree of the field extension $[\operatorname{Quot}(D): \operatorname{Quot}(C)]$, and $e$ is the multiplicity of $C$, then the following statements hold.

(1) $r e=d$.

(2) The morphism $\mathbb{P}^{1} \rightarrow \mathbb{P}^{n-1}$, which is given by $q \mapsto\left[g_{1}(q): \cdots: g_{n}(q)\right]$, is birational onto its image if and only if $r=1$.

(3) There exist forms $f_{1}$ and $f_{2}$ of degree $r$ in $B$ such that $C \subseteq k\left[f_{1}, f_{2}\right]$. In particular, $I$ is extended from an ideal in $\boldsymbol{k}\left[f_{1}, f_{2}\right]$ in the sense that $I=\left(I \cap \boldsymbol{k}\left[f_{1}, f_{2}\right]\right) B$. Furthermore, $f_{i}=\operatorname{gcd}\left(I_{1}\left(p_{i} \varphi\right)\right)$ for general points $p_{1}$ and $p_{2}$ in $\mathbb{P}^{n}$. 
In the context of Theorem 0.10 it is clear that some homogeneous Hilbert-Burch matrix for $\left[g_{1}, \ldots, g_{n}\right]$ has entries in $k\left[f_{1}, f_{2}\right]$. We identify a sufficient condition which guarantees that every Hilbert-Burch matrix for $\left[g_{1}, \ldots, g_{n}\right]$ has entries in $\boldsymbol{k}\left[f_{1}, f_{2}\right]$.

Observation 0.11. Retain the notation and hypotheses of Theorem 0.10. Assume that every entry of $\varphi$ has the same degree $c$. The following statements hold.

(1) Every entry of $\varphi$ is in the ring $\boldsymbol{k}\left[f_{1}, f_{2}\right]$.

(2) If $c$ is prime, then the morphism of Theorem 0.10 is birational if and only if $\mu\left(I_{1}(\varphi)\right) \geq 3$.

Proof. (1) Let $\boldsymbol{k}\left[z_{1}, z_{2}\right]$ be a new polynomial ring and $\theta: \boldsymbol{k}\left[z_{1}, z_{2}\right] \rightarrow \boldsymbol{k}\left[f_{1}, f_{2}\right]$ be the $\boldsymbol{k}$-algebra homomorphism which is defined by $\theta\left(z_{i}\right)=f_{i}$. Identify $g_{i}^{\prime}$ in $\boldsymbol{k}\left[z_{1}, z_{2}\right]$ with $\theta\left(g_{i}^{\prime}\right)=g_{i}$ for all $i$. It is clear that the ideal $\left(g_{1}^{\prime}, \ldots, g_{n}^{\prime}\right)$ of $\boldsymbol{k}\left[z_{1}, z_{2}\right]$ has height two. Let $\varphi^{\prime}$, with entries in $\boldsymbol{k}\left[z_{1}, z_{2}\right]$, be any homogeneous Hilbert-Burch matrix for $\left[g_{1}^{\prime}, \ldots, g_{n}^{\prime}\right]$. It follows that $\theta\left(\varphi^{\prime}\right)$ is a Hilbert-Burch matrix for $\left[g_{1}, \ldots, g_{n}\right]=$ $\theta\left(\left[g_{1}^{\prime}, \ldots, g_{n}^{\prime}\right]\right)$. Thus, there exist homogeneous invertible matrices $\chi$ and $\xi$ with entries in $B$ so that $\chi \theta\left(\varphi^{\prime}\right) \xi=\varphi$. Degree considerations show that the entries of $\chi$ and $\xi$ are actually in $\boldsymbol{k}$; and therefore, $\varphi=\chi \theta\left(\varphi^{\prime}\right) \xi=\theta\left(\chi \varphi^{\prime} \xi\right)$.

(2) The direction $(\Rightarrow)$ is obvious. We prove $(\Leftarrow)$. Assume that $\mu\left(I_{1}(\varphi)\right) \geq 3$. Every entry of $\varphi$ is a homogeneous form of degree, say $s$, in the ring $\boldsymbol{k}\left[f_{1}, f_{2}\right]$ and the homogeneous forms $f_{i}$ have degree $r$. So, the prime $c$ is equal to $r s$. The hypothesis $\mu\left(I_{1}(\varphi)\right) \geq 3$ shows that $r \neq c$. Thus, $r$ must equal 1 .

\section{Section 1. The General Lemma.}

In this section we prove Lemma 1.7, which we call the General Lemma. Let $\mathcal{C}$ be the curve which is parameterized by the homogeneous polynomials $g_{1}, \ldots, g_{n}$ of the same degree and let $p$ be the point $\left[a_{1}: \cdots: a_{n}\right]$ in $\mathbb{P}^{n-1}$. If $\varphi$ is a Hilbert-Burch matrix for $\left[g_{1}, \ldots, g_{n}\right]$, then the generalized row ideal $I_{1}\left(\left[a_{1}, \ldots, a_{n}\right] \varphi\right)$ captures a significant amount of geometric information about the behavior of $\mathcal{C}$ at $p$. Indeed, from this ideal, one may determine whether or not $p$ is on $\mathcal{C}$, the multiplicity $m_{\mathcal{C}, p}$ of $\mathcal{C}$ at $p$, the number of branches $s_{\mathcal{C}, p}$ of $\mathcal{C}$ at $p$, and the multiplicity of each branch.

The basic set-up is given in Data 1.1 and explained in Remark 1.2. Observation 1.4 is a small calculation, given originally in [14], which shows how the generic row ideal $I_{1}(p \varphi)$ can be used to determine whether or not $p$ is on $\mathcal{C}$. After stating Lemma 1.7, we immediately describe the first round of consequences; further consequences are found throughout the paper. Theorem 1.8 covers essentially the same ground as Lemma 1.7; but it might be easier to apply because the statement of Theorem 1.8 is less ambitious. Corollary 1.9, which is a result due to Song, Chen, and Goldman [30], may be deduced from Theorem 1.8. 
After giving the proof of Corollary 1.9, we lay the foundations for the proof of Lemma 1.7. Lemma 1.10 concerns the relationship between a one-dimensional local domain and its integral closure; in particular, this result is about the structure of the extension of the maximal ideal of the smaller ring. Proposition 1.12 is the statement of the well-known correspondence between the minimal prime ideals of the completion $\widehat{\mathcal{O}_{\mathcal{C}, p}}$ of the local ring $\mathcal{O}_{\mathcal{C}, p}$ and the maximal ideals of the integral closure $\overline{\mathcal{O}_{\mathcal{C}, p}}$ of $\mathcal{O}_{\mathcal{C}, p}$. Various comments about the statement of Lemma 1.7 are collected in Remarks 1.17. The proof of Lemma 1.7 is given in (1.18).

We close this section with a second application of the General Lemma: every parameterization of a curve leads to a parameterization of the branches of the curve. In particular, in Observation 1.25 and Remark 1.31, we show that if Data 1.1 is in effect, with $\boldsymbol{k}$ an algebraically closed field, then there is a one-to-one correspondence between the height one linear ideals of $B$ and the branches of $\mathcal{C}$. Indeed, it makes sense to talk about the branch " $\mathcal{C}(\ell)$ " of $\mathcal{C}$ that corresponds to the ideal $(\ell)$ of $B$, where $\ell$ is a non-zero homogeneous linear form of $B$; furthermore, it makes sense to talk about the multiplicity of the branch $\mathcal{C}(\ell)$. We return to this idea in Section 7 , where we calculate the multiplicity of the branch $\mathcal{C}(\ell)$ in terms of data found in the Jacobian ideal of the parameterization of $\mathcal{C}$.

Data 1.1. Let $k$ be a field, $g_{1}, \ldots, g_{n}$ be homogeneous forms of degree $d$ in the polynomial ring $B=k[x, y], \Psi: \mathbb{P}^{1} \rightarrow \mathbb{P}^{n-1}$ be the morphism which is given by $\left[g_{1}: \cdots: g_{n}\right], \mathcal{C}$ be the image of $\Psi$, and $I$ be the ideal $\left(g_{1}, \ldots, g_{n}\right) B$ of $B$. Assume that

(1) $I$ is minimally generated by $g_{1}, \ldots, g_{n}$,

(2) I has height two, and

(3) $\Psi: \mathbb{P}^{1} \rightarrow \mathcal{C}$ is a birational morphism.

Let $\varphi$ be a homogeneous Hilbert-Burch matrix for $\left[g_{1}, \ldots, g_{n}\right]$ and define the integers $d_{1} \leq \cdots \leq d_{n-1}$ by requiring that

$$
0 \rightarrow B\left(-d_{1}-d\right) \oplus \cdots \oplus B\left(-d_{n-1}-d\right) \stackrel{\varphi}{\longrightarrow} B(-d)^{n} \stackrel{\left[g_{1}, \ldots, g_{n}\right]}{\longrightarrow} I \rightarrow 0
$$

is a homogeneous resolution of $I$.

Remark 1.2. The hypotheses imposed on the parameterization $\Psi$ in Data 1.1 are fairly mild. Furthermore, if a given parameterization of a rational curve $\mathcal{C}$ fails to satisfy these hypotheses, one can reparameterize and obtain a parameterization of $\mathcal{C}$ which does satisfy the hypotheses. Hypothesis (1) is equivalent to the statement that $\mathcal{C} \subseteq \mathbb{P}^{n-1}$ is "non-degenerate"; that is, $\mathcal{C}$ is not contained in any hyperplane in $\mathbb{P}^{n-1}$. Hypothesis (2) is equivalent to the statement "the rational map $\Psi$ has no base points". The homogeneous coordinate ring of $\mathcal{C}$ is $\boldsymbol{k}\left[g_{1}, \ldots, g_{n}\right]=\boldsymbol{k}\left[I_{d}\right]$ and 
the homomorphism $\boldsymbol{k}\left[T_{1}, \ldots, T_{n}\right] \rightarrow \boldsymbol{k}\left[I_{d}\right]$, which sends $T_{i}$ to $g_{i}$ for each $i$, induces the isomorphism

$$
\frac{k\left[T_{1}, \ldots, T_{n}\right]}{I(\mathcal{C})} \cong \boldsymbol{k}\left[I_{d}\right]
$$

where $I(\mathcal{C})$ is the ideal generated by the homogeneous polynomials which vanish on $\mathcal{C}$. The homogeneous coordinate ring for the Veronese curve of degree $d$ is $\boldsymbol{k}\left[x^{d}, x^{d-1} y, \ldots, y^{d}\right]=\boldsymbol{k}\left[B_{d}\right]$. Hypothesis (3) is equivalent to the statement "the domains $\boldsymbol{k}\left[I_{d}\right] \subseteq \boldsymbol{k}\left[B_{d}\right]$ have the same quotient field"; see Theorem 0.10 . Theorem 0.10 also provides a procedure for producing a birational reparameterization of $\mathcal{C}$; an alternative procedure may be found in Section 6.1 of [31]. The integers $\left\{d_{i}\right\}$ could also be defined by insisting that each element of column $i$ of $\varphi$ be a homogeneous form of degree $d_{i}$. The hypotheses already in place guarantee that $1 \leq d_{1}$ and $\sum_{i=1}^{n-1} d_{i}=d$

Observation 1.4 is a small calculation, given originally in [14], which shows how the generalized row ideal $I_{1}(p \varphi)$ can be used to determine whether or not $p$ is on $\mathcal{C}$. It serves as a good introduction to the Eisenbud-Ulrich interpretation of the fibers of the morphism $\Psi$ in terms of generalized rows of the syzygy matrix $\varphi$. This calculation is expanded and fine-tuned in the proof of the General Lemma.

Observation 1.4. Adopt Data 1.1 with $\boldsymbol{k}$ algebraically closed. If $p$ is a point of $\mathbb{P}^{n-1}$, then $p$ is on $\mathcal{C}$ if and only if $\mathrm{ht}\left(I_{1}(p \varphi)\right)=1$.

Remark. Notice that we use the symbol " $p$ " to represent both a point in $\mathbb{P}^{n-1}$ and a row vector with $n$-entries from $k$; see Remark 0.8 for more explanation.

Proof. Assume first that $p$ is on $\mathcal{C}$. In this case, $p=\left[g_{1}(q): \cdots: g_{n}(q)\right]$ for some point $q \in \mathbb{P}^{1}$. The equation $\left[g_{1}, \ldots, g_{n}\right] \varphi=0$ holds by hypothesis. Therefore, the ideal

$$
I_{1}(p \varphi)=I_{1}\left(\left[g_{1}(q), \cdots, g_{n}(q)\right] \varphi\right)
$$

vanishes at $q$, and $I_{1}(p \varphi)$ is contained in the height one ideal $I(q)$ in $B$. This completes the proof of the direction $(\Rightarrow)$.

Write $p$ as $\left[a_{1}: \cdots: a_{n}\right]$ in $\mathbb{P}^{n-1}$. Consider the ideal

$$
\mathfrak{I}=I_{2}\left(\left[\begin{array}{lll}
a_{1} & \cdots & a_{n} \\
g_{1} & \cdots & g_{n}
\end{array}\right]\right)
$$

of $B$. Observe that the zero locus of $\mathfrak{I}$ is

$$
V(\mathfrak{I})=\left\{q \in \mathbb{P}^{1} \mid \Psi(q)=p\right\}
$$


which is the fiber $\Psi^{-1}(p)$ of the morphism $\Psi$ over $p$.

Let $\chi$ be an invertible matrix with entries in $\boldsymbol{k}$ and $(0, \ldots, 0,1) \chi=\left(a_{1}, \ldots, a_{n}\right)$. Define $\left[g_{1}^{\prime}, \ldots, g_{n}^{\prime}\right]=g^{\prime}$ by

$$
g^{\prime}=\left[g_{1}, \ldots, g_{n}\right] \chi^{-1}
$$

The entries of $g^{\prime}$ generate $I$ and the Hilbert-Burch matrix for $g^{\prime}$ is $\varphi^{\prime}=\chi \varphi$. One consequence of this last statement is the fact that the bottom row of $\varphi^{\prime}$ generates the ideal $\left(g_{1}^{\prime}, \ldots, g_{n-1}^{\prime}\right): g_{n}^{\prime}$. Observe that

$$
\left[\begin{array}{lll}
a_{1} & \ldots & a_{n} \\
g_{1} & \ldots & g_{n}
\end{array}\right] \chi^{-1}=\left[\begin{array}{cccc}
0 & \ldots & 0 & 1 \\
g_{1}^{\prime} & \ldots & g_{n-1}^{\prime} & g_{n}^{\prime}
\end{array}\right]
$$

and therefore, $\mathfrak{I}$ is generated by $\left(g_{1}^{\prime}, \ldots, g_{n-1}^{\prime}\right)$. We now see that

$$
I_{1}(p \varphi)=I_{1}\left([0, \ldots, 1] \varphi^{\prime}\right)=\left(g_{1}^{\prime}, \ldots, g_{n-1}^{\prime}\right): g_{n}^{\prime}=\mathfrak{I}: I .
$$

Assume $p \notin \mathcal{C}$. In this case, the zero locus $V(\mathfrak{I})$ of $\mathfrak{I}$ is empty. The field $k$ is algebraically closed; so Hilbert's Nullstellensatz ensures that $\mathfrak{I}$ is an $(x, y)$-primary ideal of $B$. We saw in (1.6) that $\mathfrak{I} \subseteq I_{1}(p \varphi)$; so, $I_{1}(p \varphi)$ is also an $(x, y)$-primary ideal. We conclude that ht $\left(I_{1}(p \varphi)\right)=2$.

Lemma 1.7. (The General Lemma). Adopt Data 1.1. Fix the point $p$ on $\mathcal{C}$. Assume that the fiber $\Psi^{-1}(p)$ is equal to the fiber $\Psi_{\overline{\boldsymbol{k}}}^{-1}(p)$, where $\Psi_{\overline{\boldsymbol{k}}}: \mathbb{P}_{\overline{\boldsymbol{k}}}^{1} \rightarrow \mathbb{P}_{\overline{\boldsymbol{k}}}^{n-1}$ is the extension of $\Psi$ to a morphism over the algebraic closure $\overline{\boldsymbol{k}}$ of $\boldsymbol{k}$. Let $q_{1}, \ldots, q_{s}$ be the $s$ distinct points in $\mathbb{P}^{1}$ which comprise the fiber $\Psi^{-1}(p) ; \mathfrak{p}$ be the prime ideal in $\boldsymbol{k}\left[I_{d}\right]$ which corresponds to the point $p$ on $\mathcal{C} ; \mathfrak{q}_{1}, \ldots, \mathfrak{q}_{s}$ be the prime ideals in $B$ which correspond to the points $q_{1}, \ldots, q_{s}$ in $\mathbb{P}^{1}$, and $\Delta$ be the greatest common divisor of the entries of the row vector $p \varphi$. Write $\Delta=\ell_{1}^{c_{1}} \cdots \ell_{t}^{c_{t}}$, where $\ell_{1}, \ldots, \ell_{t}$ are pairwise non-associate irreducible homogeneous forms in $B$. Let $R \subseteq S$ be the rings $\boldsymbol{k}\left[I_{d}\right]_{\mathfrak{p}} \subseteq B_{\mathfrak{p}}, \hat{R} \subseteq \hat{S}$ be the completions of $R \subseteq S$ in the $\mathfrak{m}_{R}$-adic topology, $J_{1}, \ldots, J_{u}$ be the minimal prime ideals of $\hat{R}$, and $\mathfrak{M}_{1}, \ldots \mathfrak{M}_{v}$ be the maximal ideals of $\hat{S}$. Then the parameters $s, t, u, v$, are all equal to $s_{\mathcal{C}, p}$ (which is the number of branches of $\mathcal{C}$ at $p$ ), and, after re-numbering,

$$
q_{i}=V\left(\ell_{i}\right), \quad \mathfrak{q}_{i}=\ell_{i} B, \quad \mathfrak{M}_{i}=\mathfrak{q}_{i} \hat{S}, \quad J_{i}=\operatorname{ker}\left(\hat{R} \rightarrow \hat{S}_{\mathfrak{M}_{i}}\right), \quad \text { and } \quad c_{i}=e\left(\hat{R} / J_{i}\right)
$$

for $1 \leq i \leq s$. In particular, $\operatorname{deg} \Delta=e(R)=e\left(\mathcal{O}_{\mathcal{C}, p}\right)=m_{\mathcal{C}, p}$, and $c_{1}, \ldots, c_{s}$ are the multiplicities of the branches of $\mathcal{C}$ at $p$.

Some remarks pertaining to the statement of Lemma 1.7 may be found in Remarks 1.17. The proof of Lemma 1.7 is given in (1.18). We proceed immediately to the first round of applications. 
Theorem 1.8. Adopt Data 1.1 with $\boldsymbol{k}$ an algebraically closed field. Let $p_{1}, \ldots, p_{z}$ be the singularities of $\mathcal{C}$. For each singular point $p_{j}$, let $m_{j}$ be the multiplicity of $\mathcal{C}$ at $p_{j}$ and $s_{j}$ be the number of branches of $\mathcal{C}$ at $p_{j}$. Then the following statements hold:

(1) The polynomial $\operatorname{gcd} I_{1}\left(p_{j} \varphi\right)$ in $\boldsymbol{k}[x, y]$ has degree equal to $m_{j}$ and has $s_{j}$ pairwise non-associated linear factors.

(2) The polynomials $\operatorname{gcd} I_{1}\left(p_{i} \varphi\right)$ and $\operatorname{gcd} I_{1}\left(p_{j} \varphi\right)$ are relatively prime for $i \neq j$.

Proof. Assertion (1) is explicitly stated as part of Lemma 1.7. We prove (2). Lemma 1.7 shows that $\operatorname{gcd} I_{1}\left(p_{j} \varphi\right)=\prod_{u=1}^{s_{j}} \ell_{u, j}^{e_{u, j}}$, where the linear factors $\ell_{u, j}$ correspond to the points in the fiber $\Psi^{-1}\left(p_{j}\right)$. If $i \neq j$, then the fibers $\Psi^{-1}\left(p_{i}\right)$ and $\Psi^{-1}\left(p_{j}\right)$ are disjoint, so; the polynomials $\operatorname{gcd} I_{1}\left(p_{i} \varphi\right)$ and $\operatorname{gcd} I_{1}\left(p_{j} \varphi\right)$ are relatively prime.

As our first application of Theorem 1.8 we recover results of Song, Chen, and Goldman. The language and techniques of [30] are much different from ours; nonetheless, [30, Theorem 3] is essentially the same as the next result. Indeed, the General Lemma is our first attempt to interpret the results of [30] in terms of generalized row ideals.

Corollary 1.9. Adopt Data 1.1 with $\boldsymbol{k}$ an algebraically closed field and $n=3$, and let $p$ be a point on $\mathcal{C}$. The following statements hold.

(1) The multiplicity of $\mathcal{C}$ at $p$ satisfies $m_{p} \leq d_{2}$.

(2) If $m_{p}<d_{2}$, then $m_{p} \leq d_{1}$.

(3) The curve $\mathcal{C}$ has a singularity of multiplicity $d-1$ if and only if $d_{1}=1$.

(4) The multiplicity of $\mathcal{C}$ at $p$ is equal to $d_{j}$, for $j$ equal to 1 or 2 , if and only if there exist homogeneous invertible matrices $\chi$ and $\xi$ (the entries of $\chi$ are in $\boldsymbol{k}$, the entries of $\xi$ are in $B)$ such that $p=[0: 0: 1] \chi$ and $\chi \varphi \xi$ is equal to

$$
\varphi^{\prime}=\left[\begin{array}{cc}
P_{1} & Q_{1} \\
P_{2} & Q_{2} \\
0 & Q_{3}
\end{array}\right]
$$

where the $P_{i}$ and the $Q_{i}$ are homogeneous forms from $B$ with $\operatorname{deg} P_{i}=d-d_{j}$, and $\operatorname{deg} Q_{i}=d_{j}$. Furthermore, in this situation, the matrices $\chi$ and $\xi$ may be chosen so that $\operatorname{gcd}\left(P_{1}, Q_{1}\right)=1$ and $\operatorname{gcd}\left(P_{2}, Q_{2}\right)=1$.

Remark. The invertible matrix $\chi$ from (4) gives rise to a linear automorphism of $\mathbb{P}^{2}$ which sends $p$ to $[0: 0: 1]$ as described in Remark 0.9 .

Proof. Let $p \varphi$ be the row vector $\left[a_{1}, a_{2}\right]$. The hypotheses tell us that

$$
\operatorname{deg} a_{1}=d_{1} \leq d_{2}=\operatorname{deg} a_{2} .
$$


Theorem 1.8 now gives

$$
m_{p}=\operatorname{deg} \operatorname{gcd}\left(a_{1}, a_{2}\right) \leq d_{2}
$$

and (1) is established. If $m_{p}<d_{2}$, then $a_{1} \neq 0$, and

$$
m_{p}=\operatorname{deg} \operatorname{gcd}\left(a_{1}, a_{2}\right) \leq \operatorname{deg} a_{1}=d_{1},
$$

and (2) is established. We now prove (3). If $p^{\prime}$ is a point on $\mathcal{C}$ with $m_{p^{\prime}}=d-1$, then (1) shows that

$$
d-1=m_{p^{\prime}} \leq d_{2}=d-d_{1} \leq d-1 \text {. }
$$

Thus, $m_{p^{\prime}}=d-1 \Longrightarrow d_{1}=1$. Conversely, if $d_{1}=1$, then the entries of column 1 of $\varphi$ span the two-dimensional vector space $B_{1}$; hence, there exists a point $p^{\prime}$ in $\mathbb{P}^{2}$ so that the left entry of $p^{\prime} \varphi$ is zero. The ideal $\left(g_{1}, g_{2}, g_{3}\right)$ is 3 -generated; so the ideal $I_{1}\left(p^{\prime} \varphi\right)$ is non-zero and is generated by a homogeneous form of degree $d-1$. Lemma 1.7 (together with Observation 1.4) show that $p^{\prime}$ is a singularity on $\mathcal{C}$ of multiplicity $d-1$.

Finally, we prove (4). Assume that $m_{p}=d_{j}$. It follows that

$$
\operatorname{deg} a_{j}=d_{j}=m_{p}=\operatorname{deg} \operatorname{gcd}\left(a_{1}, a_{2}\right)
$$

and the ideal $\left(a_{1}, a_{2}\right)$ of $B$ must be principal. Thus, there are homogeneous invertible matrices $\chi$ and $\xi$ so that $(0,0,1) \chi=p$ and $\chi \varphi \xi$ has the form

$$
\varphi^{\prime}=\left[\begin{array}{cc}
P_{1} & Q_{1} \\
P_{2} & Q_{2} \\
0 & Q_{3}
\end{array}\right]
$$

The maximal minors of $\varphi^{\prime}$ generate an ideal of height two; hence, $P_{1}, Q_{1}, Q_{3}$ have no factor in common. One may add a scalar multiple of row three to row one in order to ensure that the entries of row one are relatively prime. The analogous argument works for row two. The later rounds of row operations do not change the bottom row of $\chi$.

Remark. The method of [30] (see also [10, Theorem 1]) shows that a rational plane curve of degree $d$ can have at most one singularity of multiplicity greater than $d / 2$. This is clear by Bézout's Theorem and is also implied by our method since one can not create two zeros in a column of a Hilbert-Burch matrix without violating the height condition.

We next collect the preliminary results (Lemmas 1.10 and 1.16, and Proposition 1.12) which are used in the proof of Lemma 1.7. 
Lemma 1.10. Let $\left(A, \mathfrak{m}_{A}, \boldsymbol{k}_{A}\right) \subseteq\left(B, \mathfrak{m}_{B}, \boldsymbol{k}_{B}\right)$ be one-dimensional local domains. Assume that $\boldsymbol{k}_{A}$ is infinite. If $B$ is the integral closure of $A$ and $B$ is finitely generated as an $A$-module, then $\mathfrak{m}_{A} B=\mathfrak{m}_{B}^{e / r}$, where $e=e(A)$ is the multiplicity of the local ring $A$ and $r=\left[\boldsymbol{k}_{B}: \boldsymbol{k}_{A}\right]$ is the degree of the field extension $\boldsymbol{k}_{A} \subseteq \boldsymbol{k}_{B}$.

Proof. The hypothesis about $\boldsymbol{k}_{A}$ ensures that there exists a minimal reduction of $\mathfrak{m}_{A}$ generated by one element $z$ and that $e=\lambda_{A}(A / z A)$; see, for example, $[32$, Prop. 11.2.2]. The domain $B$ is normal, local, and one dimensional; so, $B$ is a Principal Ideal Domain and the equation $z \mathfrak{m}_{A}^{u} B=\mathfrak{m}_{A}^{u+1} B$, for some $u$, tells us that $z B=\mathfrak{m}_{A} B$. We compute

$$
e=\lambda_{A}(A / z A)=\lambda_{A}(B / z B)=\lambda_{B}(B / z B) r
$$

The middle equality is obtained from the picture

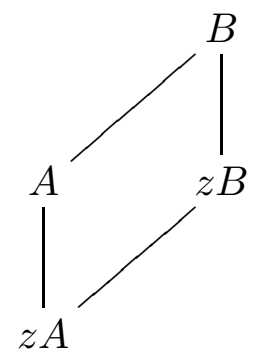

All lengths are finite; in particular, $\lambda_{A}(B / A)$ is finite because $A$ is a one-dimensional domain and $B$ is a module-finite extension of $A$ with $B \subseteq \operatorname{Quot}(A)$. Multiplication by $z$ gives an isomorphism of $A$-modules $B / A \cong z B / z A$; therefore the $A$-modules $A / z A$ and $B / z B$ have the same length. The equality on the right is due to the fact that every factor in a composition series for the $B$-module $B / z B$ is $\boldsymbol{k}_{B}$. The formulas of (1.11) have been established and $\lambda_{B}(B / z B)=e / r$. The only quotient of $B$ with length $e / r$, as a $B$-module, is $B / \mathfrak{m}_{B}^{e / r}$. We conclude that $\mathfrak{m}_{A} B=z B=$ $\mathfrak{m}_{B}^{e / r}$.

Proposition 1.12 is the well-known correspondence between the minimal prime ideals of the completion $\widehat{\mathcal{O}_{\mathcal{C}, p}}$ of the local ring $\mathcal{O}_{\mathcal{C}, p}$ and the maximal ideals of the integral closure $\overline{\mathcal{O}_{\mathcal{C}, p}}$ of $\mathcal{O}_{\mathcal{C}, p}$. This result is stated as Exercise 1 on page 122 in Nagata [28]; see [21, Corollary 5] and [23, Thm. 16.14] for a proof.

Proposition 1.12. Let $T$ be the integral closure of the one-dimensional local domain $(R, \mathfrak{m})$. Suppose $T$ is finitely generated as a module over $R$. Let $\hat{R}$ and $\hat{T}$ represent the completions of $R$ and $T$ in the $\mathfrak{m}$-adic topology. Then there is a one-to-one correspondence between the minimal prime ideals of $\hat{R}$ and the maximal 
ideals of $T$. If $\mathcal{M}$ is a maximal ideal of $T$, then the corresponding minimal prime ideal of $\hat{R}$ is $\operatorname{ker}\left(\hat{R} \rightarrow \hat{T}_{\mathcal{M} \hat{T}}\right)$.

Remark 1.13. When the hypotheses of Proposition 1.12 are in effect then $\hat{T}$ is the integral closure of $\hat{R}$. Indeed, $\hat{T}$ is a normal ring which is finitely generated as a module over $\hat{R}$. Lemma 1.14 shows that $\hat{T} \subseteq$ Quot $\hat{R}$.

Lemma 1.14. Let $R \subseteq T \subseteq \operatorname{Quot}(R)$ be rings with $(R, \mathfrak{m})$ a local domain and $T$ a finitely generated $R$-module. Let $\hat{R}$ and $\hat{T}$ be the completions of $R$ and $T$ in the $\mathfrak{m}$-adic topology. Then $\hat{R}$ and $\hat{T}$ have the same total quotient ring.

Proof. Let $U=R \backslash\{0\}$. Notice first that $\operatorname{Quot}(R)=U^{-1} R=U^{-1} T$. Every element of $U$ is regular on both rings $\hat{R} \subseteq \hat{T}$; so, $U^{-1}(\hat{R}) \subseteq U^{-1}(\hat{T}) \subseteq \operatorname{Quot}(\hat{T})$. The rings $U^{-1}(\hat{R}) \subseteq U^{-1}(\hat{T})$ are equal because

$$
\begin{aligned}
U^{-1}(\hat{T}) & =U^{-1}\left(T \otimes_{R} \hat{R}\right)=U^{-1}(T) \otimes_{U^{-1}(R)} U^{-1}(\hat{R})=U^{-1}(R) \otimes_{U^{-1}(R)} U^{-1}(\hat{R}) \\
& =U^{-1}(\hat{R})
\end{aligned}
$$

We have $U^{-1} \hat{T}=U^{-1} \hat{R} \subseteq$ Quot $\hat{T}$. A typical element of Quot $\hat{T}$ is $z / w$, where $z$ and $w$ are in $\hat{T}$ with $w$ regular on $\hat{T}$. There exists $u \in U$ with $u z, u w$ in $\hat{R}$. Of course $u w$ is regular on $\hat{R} \subseteq \hat{T}$. So, $z / w=u z / u w \in$ Quot $\hat{R}$.

In Lemma 1.16 we show that if $A$ is a complete local domain and $z$ is an indeterminate over $A$, then the local ring $A(z)$, see (0.6), is analytically irreducible. Our proof uses the fact that the completion of an excellent normal ring is a normal ring. We will apply Lemma 1.16 in the proof of Lemma 1.7 to calculate the multiplicity of a branch of a curve.

Observation 1.15. If $A \subseteq C$ is a module finite extension of local rings and $z$ is an indeterminate over $C$, then $A(z) \subseteq C(z)$ is module finite extension of local rings.

Proof. Let $\mathfrak{m}_{A}$ and $\mathfrak{m}_{C}$ be the maximal ideals of $A$ and $C$, respectively. The CohenSeidenberg Theorems ensure that $\mathfrak{m}_{C}$ is the only prime ideal of $C$ which contracts to $\mathfrak{m}_{A}$; so, in particular, the radical of the ideal $\mathfrak{m}_{A} C$ of $C$ is $\operatorname{rad} \mathfrak{m}_{A} C=\mathfrak{m}_{C}$, and the ideal $\mathfrak{m}_{C} C[z]$ contracts to $\mathfrak{m}_{A} A[z]$.

Consider the multiplicatively closed subsets

$$
S=A[z] \backslash \mathfrak{m}_{A} A[z] \subseteq T=C[z] \backslash \mathfrak{m}_{C} C[z]
$$

of $C[z]$. We know that $S^{-1}(A[z]) \subseteq S^{-1}(C[z])$ is a module finite extension, with $A(z)=S^{-1}(A[z])$. Moreover, $C(z)=T^{-1}(C[z])$ is a further localization of $S^{-1}(C[z])$ at a maximal ideal of $S^{-1}(C[z])$. To prove the result, it suffices to 
show that $C(z)$ is already equal to $S^{-1}(C[z])$; and therefore, it suffices to show that $S^{-1}(C[z])$ is already a local ring.

The inclusion $A(z) \subseteq S^{-1}(C[z])$ is a module finite extension and $A(z)$ is a local ring with maximal ideal $\mathfrak{m}_{A} A(z)$. According to the Cohen-Seidenberg Theorems, the maximal spectrum of $S^{-1}(C[z])$ is contained in

$$
\left\{P S^{-1}(C[z]) \mid P \text { is a prime ideal of } C[z] \text {, minimal over } \mathfrak{m}_{A} C[z]\right\} \text {. }
$$

The above set consists of exactly one ideal because $\operatorname{rad}\left(\mathfrak{m}_{A} C\right) C[z]$ is a prime ideal of $C[z]$ since

$$
\frac{C[z]}{\operatorname{rad}\left(\mathfrak{m}_{A} C[z]\right)}=\frac{C}{\operatorname{rad}\left(\mathfrak{m}_{A} C\right)}[z]=\frac{C}{\mathfrak{m}_{C}}[z]
$$

which is a domain. Thus, $S^{-1}(C[z])$ is a local ring and the proof is complete.

Lemma 1.16. If $A$ is a complete local domain and $z$ is an indeterminate over $A$, then the completion $A(z)^{\wedge}$ of the local ring $A(z)$ is also a domain.

Proof. Let $V$ be the integral closure of $A$. Our proof amounts to showing that each vertical map in the commutative diagram of rings:

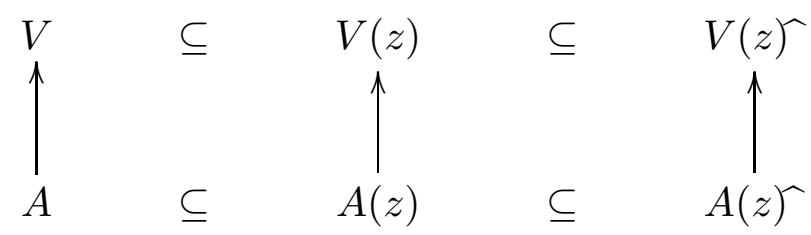

is a module-finite inclusion. The $\operatorname{ring}\left(A, \mathfrak{m}_{A}\right)$ is local, Noetherian, and complete; hence excellent; see, for example, [27, pg. 260]. It follows that the integral closure $V$ of $A$ is finitely generated as an $A$-module. The ring $V$ is necessarily local. Indeed, $V$ is semi-local and complete in the $\mathfrak{m}_{A}$-adic topology; hence, $V$ is a direct product of local rings. On the other hand, $V$ is a domain since it is contained in the quotient field of $A$ and so only one local ring appears in the previously mentioned direct product decomposition of $V$. Apply Observation 1.15 to see that $V(z)$ is finitely generated as an $A(z)$-module. Let $V(z)^{\wedge}$ be the completion of the local ring $V(z)$. We now see that the natural map $A(z)^{\uparrow} \rightarrow V(z)^{\Upsilon}$ is an injection; since $V(z)^{\wedge}=V(z) \otimes_{A(z)} A(z)^{\Upsilon}$ and the completion map $A(z) \rightarrow A(z)^{\Upsilon}$ is flat. The rings $V$ and $V(z)$ are excellent normal local rings and therefore the completion $V(z)^{\Upsilon}$ is also an excellent normal local ring; see, for example, [27, Thm 79]. The local normal ring $V(z)^{\Upsilon}$ is necessarily a normal domain and the subring $A(z)^{\Upsilon}$ of $V(z)^{\Upsilon}$ is also a domain.

The following remarks pertain to the statement of Lemma 1.7. 
Remarks 1.17. (a) We use the symbol $p$ to represent a point $\left[a_{1}: \cdots: a_{n}\right]$ in $\mathbb{P}^{n-1}$ as well as a row vector $\left[a_{1}, \ldots, a_{n}\right]$. The meaning will be clear from context; see Remark 0.8.

(b) The important conclusions are $s=t, \mathfrak{q}_{i}=\ell_{i} B$, and $c_{1}, \ldots, c_{s}$ are the multiplicities of the branches of $\mathcal{C}$ at $p$. The other statements are well-known, or follow easily from these, as we explain below.

(c) The prime ideal in $k\left[T_{1}, \ldots, T_{n}\right]$ which corresponds to the point $p$ in $\mathbb{P}^{n-1}$ is $I_{2}(M)$ for

$$
M=\left(\begin{array}{lll}
a_{1} & \ldots & a_{n} \\
T_{1} & \ldots & T_{n}
\end{array}\right) .
$$

The prime ideal $\mathfrak{p}$ in $\boldsymbol{k}\left[I_{d}\right]$ which corresponds to $p$ on $\mathcal{C}$ is $I_{2}(M) \boldsymbol{k}\left[I_{d}\right]$, see (1.3); thus,

$$
\mathfrak{p}=I_{2}\left(\begin{array}{ccc}
a_{1} & \ldots & a_{n} \\
g_{1} & \ldots & g_{n}
\end{array}\right) \boldsymbol{k}\left[I_{d}\right]
$$

(d) Recall that $\mathfrak{p}$ is a prime ideal of the ring $\boldsymbol{k}\left[I_{d}\right], R=\boldsymbol{k}\left[I_{d}\right]_{\mathfrak{p}}$, and $S=B_{\mathfrak{p}}$. Our notation means that $S$ is the localization $U^{-1} B$ of $B$ at the multiplicatively closed set $U=\boldsymbol{k}\left[I_{d}\right] \backslash \mathfrak{p}$. Let $T=\boldsymbol{k}\left[B_{d}\right]_{\mathfrak{p}}$. (In other words, $T$ is equal to $U^{-1}\left(\boldsymbol{k}\left[B_{d}\right]\right)$.) The ring inclusions $\boldsymbol{k}\left[I_{d}\right] \subseteq \boldsymbol{k}\left[B_{d}\right] \subseteq B$ are integral extensions; so the ring inclusions $R \subseteq T \subseteq S$ are integral extensions. The Veronese ring $k\left[B_{d}\right]$ is a normal domain and the domains $k\left[I_{d}\right] \subseteq \boldsymbol{k}\left[B_{d}\right]$ have the same quotient field by hypothesis (3) of Data 1.1; hence, $\boldsymbol{k}\left[B_{d}\right]$ is the integral closure of $\boldsymbol{k}\left[I_{d}\right]$ and $T$ is the integral closure of $R$.

(e) The ring inclusions $R \subseteq T \subseteq S$ are module finite extensions and $R$ is a local ring with maximal ideal $\mathfrak{m}_{R}=\mathfrak{p} k\left[I_{d}\right]_{\mathfrak{p}}$. It follows from the Cohen-Seidenberg Theorems that $T$ and $S$ are semi-local rings. Moreover, Proj $\boldsymbol{k}\left[B_{d}\right]=\operatorname{Proj} B$; so the function $M \mapsto M \cap T$ gives a one-to-one correspondence between the maximal ideals of $S$ and the maximal ideals of $T$.

(f) The $\mathfrak{m}_{R}$-adic topology on $S$ is equivalent to the $J$-adic topology on $S$, where $J$ is the Jacobson radical of $S$. It is well known (see, for example, [26, Thm. 8.15] or $[23$, Thm. K.11]) that the natural map

$$
\hat{S} \rightarrow \hat{S}_{\mathfrak{M}_{1}} \times \cdots \times \hat{S}_{\mathfrak{M}_{v}}
$$

is an isomorphism; furthermore, the local ring $\hat{S}_{\mathfrak{M}_{i}}$ is complete for each $i$ and $\hat{S}_{\mathfrak{M}_{i}}$ is equal to the completion of the local ring $S_{\mathfrak{M}_{i} \cap S}$ in the $\left(\mathfrak{M}_{i} \cap S\right)$-adic topology. Each ring $S_{\mathfrak{M}_{i} \cap S}$ is a one-dimensional regular ring; hence, each $\hat{S}_{\mathfrak{M}_{i}}$ is a complete DVR. Furthermore, the maximal ideals of $S$ are $\left\{\mathfrak{M}_{i} \cap S \mid 1 \leq i \leq v\right\}$. 
(g) The statements of Remark (f) also apply to $T$. So there is a commutative diagram

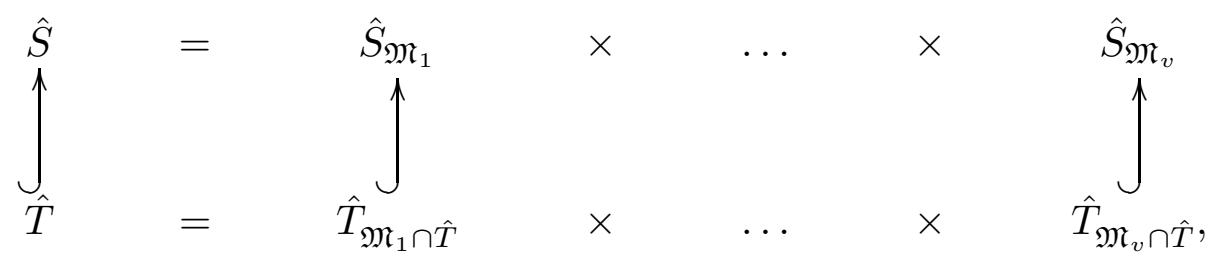

where $\hat{T}_{\mathfrak{M}_{i} \cap \hat{T}}$ is the localization of the ring $\hat{T}$ at the maximal ideal $\mathfrak{M}_{i} \cap \hat{T}$ and also is the completion of the local ring $T_{\mathfrak{M}_{i} \cap T}$ at the maximal ideal $\mathfrak{M}_{i} \cap T$. Each map $\hat{T}_{\mathfrak{M}_{i} \cap \hat{T}} \hookrightarrow \hat{S}_{\mathfrak{M}_{i}}$ is an integral extension.

(h) Proposition 1.12 may be applied to the rings $R \subseteq T$ in order to see that $u=v$, and, after renumbering, $J_{i}=\operatorname{ker}\left(\hat{R} \rightarrow \hat{T}_{\mathfrak{M}_{i} \cap \hat{T}}\right)$. Also, $R$ is a reduced local ring which is the localization of a finitely generated algebra over a field; so $\hat{R}$ is reduced; see, [35, Vol. 2, Chapt. 8, Sect. 13] or [32, page 177, item (1)]. We enlarge the commutative diagram of $(\mathrm{g})$ to obtain the commutative diagram:

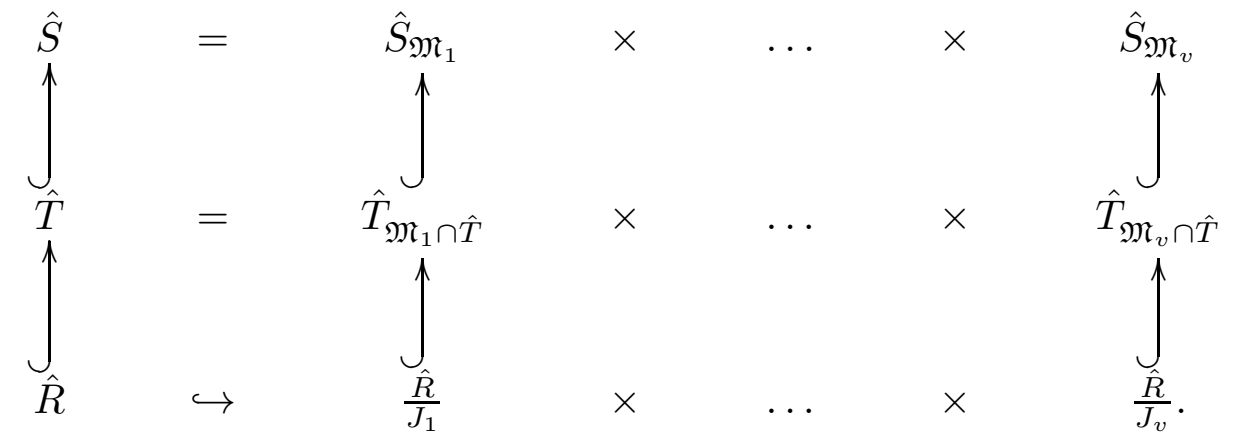

The ring $\hat{T}$ is the integral closure of $\hat{R}$ by Remark 1.13; and therefore, for each $i$, the ring $\hat{T}_{\mathfrak{M}_{i} \cap \hat{T}}$ is the integral closure of $\frac{\hat{R}}{J_{i}}$.

(1.18) Proof of Lemma 1.7. The ring extension $k\left[I_{d}\right] \subseteq B$ is integral; so every maximal ideal of $S$ has the form $\mathfrak{q} S$, where $\mathfrak{q}$ is a height one homogeneous prime ideal of $B$ which is minimal over $\mathfrak{p} B$ and which satisfies $\mathfrak{q} \cap \boldsymbol{k}\left[I_{d}\right]=\mathfrak{p}$. Let $\mathfrak{q}$ be a homogeneous prime ideal in $B$ for which $\mathfrak{q} S$ is a maximal ideal of $S$. The ideal $\mathfrak{q}$ is principal and is generated by some homogeneous form $f \in B$. Let $q \in \mathbb{P}_{\overline{\boldsymbol{k}}}^{1}$ be a root of $f$. The generators of $\mathfrak{p}$, which may be found in Remark 1.17 (c), are in the ideal $\mathfrak{q}=(f)$; and therefore, the generators of $\mathfrak{p}$ all vanish at $q$. It follows that $\Psi_{\overline{\boldsymbol{k}}}(q)=p$. The hypothesis $\Psi_{\bar{k}}^{-1}(p)=\Psi^{-1}(p)$ ensures that $q$ is already in $\mathbb{P}^{1}$; and therefore, $q \in\left\{q_{1}, \ldots, q_{s}\right\}, f$ is a linear polynomial, and $\mathfrak{q} \in\left\{\mathfrak{q}_{1}, \ldots, \mathfrak{q}_{s}\right\}$. We conclude that

$$
\text { the maximal ideals of } S \text { are }\left\{\mathfrak{q}_{1} S, \ldots, \mathfrak{q}_{s} S\right\} \text {. }
$$


It follows from Remark 1.17 (f) that $v=s$ and the maximal ideals of $\hat{S}$ are $\mathfrak{M}_{i}=$ $\mathfrak{q}_{i} \hat{S}$, for $1 \leq i \leq s$.

We saw in (1.6) that

$$
\mathfrak{p} B: I=I_{1}(p \varphi)
$$

Fix $g^{\prime}=g \chi$, as described in (1.5), with $\mathfrak{p}=\left(g_{1}^{\prime}, \ldots, g_{n-1}^{\prime}\right)$. We compute the saturation $\mathfrak{p} B:(x, y)^{\infty}$ two different ways. On the one hand, $\mathfrak{p} B:(x, y)^{\infty}$ is equal to the intersection of the $\mathfrak{q}$-primary components of $\mathfrak{p} B$ as $\mathfrak{q}$ roams over all of the height one prime ideals of $B$ in Ass $B / \mathfrak{p} B$. For each such $\mathfrak{q}$, the $\mathfrak{q}$-primary component of $\mathfrak{p} B$ is $\mathfrak{p} B_{\mathfrak{q}} \cap B$ and the ring $B_{\mathfrak{q}}$ is a DVR; so, $\mathfrak{p} B_{\mathfrak{q}}=\mathfrak{q}^{w} B_{\mathfrak{q}}$ for some exponent $w$. Thus,

$$
\mathfrak{p} B:(x, y)^{\infty}=\mathfrak{q}_{1}^{\left(w_{1}\right)} \cap \cdots \cap \mathfrak{q}_{s}^{\left(w_{s}\right)}=\mathfrak{q}_{1}^{w_{1}} \cap \cdots \cap \mathfrak{q}_{s}^{w_{s}}=\mathfrak{q}_{1}^{w_{1}} \cdots \mathfrak{q}_{s}^{w_{s}}
$$

We have taken advantage of the fact that each $\mathfrak{q}_{i}$ is principal in the Unique Factorization Domain $B$. On the other hand, the ideal $I$ is $(x, y)$-primary and ": is associative, so we use (1.20) to see that

$$
\begin{aligned}
\mathfrak{p} B:(x, y)^{\infty} & =\mathfrak{p} B: I^{\infty}=(\mathfrak{p} B: I): I^{\infty}=I_{1}(p \varphi): I^{\infty}=I_{1}(p \varphi):(x, y)^{\infty}=\Delta B \\
& =\ell_{1}^{c_{1}} \cdots \ell_{t}^{c_{t}} B .
\end{aligned}
$$

We have two factorizations of $\Delta$ into non-associate irreducible factors. We conclude that $s=t$, and after renumbering, $\ell_{i} B=\mathfrak{q}_{i}$ and $w_{i}=c_{i}$. It follows that each $\ell_{i}$ is a linear form and $q_{i}=V\left(\ell_{i}\right)$.

We next calculate the multiplicity $e\left(\hat{R} / J_{i}\right)$ for each $i$. Recall that the maximal ideals of $T$ are

$$
\left(\mathfrak{M}_{i} \cap \hat{T}\right) \cap T=\left(\mathfrak{q}_{i} \cap \boldsymbol{k}\left[B_{d}\right]\right) T \quad \text { for } 1 \leq i \leq s
$$

and that the completion $\left(T_{\left(\mathfrak{q}_{i} \cap k\left[B_{d}\right]\right) T}\right)^{\varsigma}$ of the local ring $T_{\left(\mathfrak{q}_{i} \cap \boldsymbol{k}\left[B_{d}\right]\right) T}$ at the maximal ideal $\left(\mathfrak{q}_{i} \cap \boldsymbol{k}\left[B_{d}\right]\right) T_{\left(\mathfrak{q}_{i} \cap \boldsymbol{k}\left[B_{d}\right]\right) T}$ is equal to the localization $\hat{T}_{\mathfrak{M}_{i} \cap \hat{T}}$ of the complete ring $\hat{T}$ at the maximal ideal $\mathfrak{M}_{i} \cap \hat{T}$. We simplify the notation by letting

$R_{i}=\hat{R} / J_{i}, T_{i}=\left(T_{\left(\mathfrak{q}_{i} \cap \boldsymbol{k}\left[B_{d}\right]\right) T}\right)^{\wedge}=\hat{T}_{\mathfrak{M}_{i} \cap \hat{T}}$, and $\mathfrak{m}_{T_{i}}=\left(\mathfrak{q}_{i} \cap \boldsymbol{k}\left[B_{d}\right]\right) T_{i}=\left(\mathfrak{M}_{i} \cap \hat{T}\right) T_{i}$

Recall from Remark $1.17(\mathrm{~h})$ that $T_{i}$ is the integral closure $\bar{R}_{i}$ of $R_{i}$. The maximal ideal of $R_{i}$ is

$$
\mathfrak{m}_{R_{i}}=\mathfrak{m}_{\hat{R} / J_{i}}=\mathfrak{m}_{\hat{R}} / J_{i}=\left(\mathfrak{m}_{R} \hat{R}\right) / J_{i}=((\mathfrak{p} R) \hat{R}) / J_{i}=(\mathfrak{p} \hat{R}) / J_{i}
$$


We know (see Remark $1.17(\mathrm{~h}))$ that $J_{i}=\operatorname{ker}\left(\hat{R} \rightarrow T_{i}\right)$; so,

$$
\mathfrak{m}_{R_{i}} T_{i}=\mathfrak{p} T_{i}
$$

The ideal $\mathfrak{p} T_{i}$ is completely determined by the $\left(\mathfrak{q}_{i} \cap \boldsymbol{k}\left[B_{d}\right]\right)$-primary component of $\mathfrak{p} k\left[B_{d}\right]$. We have already computed the primary components of $\mathfrak{p} B$ corresponding to the prime ideals minimal in the support of $B / \mathfrak{p} B$ :

$$
\mathfrak{p} B:(x, y)^{\infty}=\mathfrak{q}_{1}^{c_{1}} \cap \cdots \cap \mathfrak{q}_{s}^{c_{s}}
$$

The rings $\boldsymbol{k}\left[B_{d}\right]_{\mathfrak{q}_{i} \cap \boldsymbol{k}\left[B_{d}\right]} \subseteq B_{\mathfrak{q}_{i}}$ are DVRs. One can choose the same generator for the maximal ideal of these two rings. There is no difficulty in seeing that if $f$ is an element of $\boldsymbol{k}\left[B_{d}\right]$ and $r$ is arbitrary, then

$$
f \in\left(\mathfrak{q}_{i} \cap \boldsymbol{k}\left[B_{d}\right]\right)^{r} \boldsymbol{k}\left[B_{d}\right]_{\mathfrak{q}_{i} \cap \boldsymbol{k}\left[B_{d}\right]} \Longleftrightarrow f \in \mathfrak{q}_{i}^{r} B_{\mathfrak{q}_{i}}
$$

and therefore, there is no difficulty in seeing that the $\left(\mathfrak{q}_{i} \cap \boldsymbol{k}\left[B_{d}\right]\right)$-primary component of $\mathfrak{p} \boldsymbol{k}\left[B_{d}\right]$ is $\left(\mathfrak{q}_{i} \cap \boldsymbol{k}\left[B_{d}\right]\right)^{c_{i}}$. It follows from (1.21) that

$$
\mathfrak{m}_{R_{i}} T_{i}=\mathfrak{p} T_{i}=\mathfrak{m}_{T_{i}}^{c_{i}} T_{i}
$$

On the other hand, Lemma 1.10 shows that $\mathfrak{m}_{R_{i}} T_{i}=\mathfrak{m}_{T_{i}}^{e\left(R_{i}\right)} T_{i}$ because $R_{i} \subseteq T_{i}$ are local one-dimensional domains with common residue class field $k\left(g_{n}^{\prime}\right) \subseteq R_{i}$, for $g_{n}^{\prime}$ as found in (1.5); furthermore, $T_{i}$ is the integral closure of $R_{i}$ and $T_{i}$ is finitely generated as an $R_{i}$-module. Thus,

$$
\mathfrak{m}_{T_{i}}^{c_{i}} T_{i}=\mathfrak{m}_{R_{i}} T_{i}=\mathfrak{m}_{T_{i}}^{e\left(R_{i}\right)} T_{i}
$$

and $c_{i}=e\left(R_{i}\right)$

Next, we relate the degree of the polynomial $\Delta$ to the multiplicity of the local ring $R$. The modules $R / \mathfrak{m}_{R}^{r}$ and $\hat{R} / \mathfrak{m}_{R}^{r} \hat{R}$ are equal for all $r$; so $e(R)=e(\hat{R})$. The associativity formula for multiplicities yields

$$
e(\hat{R})=\sum_{i=1}^{s} e\left(\hat{R} / J_{i}\right)
$$

Thus,

$$
e(R)=e(\hat{R})=\sum_{i=1}^{s} e\left(\hat{R} / J_{i}\right)=\sum_{i=1}^{s} c_{i}=\operatorname{deg}\left(\ell_{1}^{c_{1}} \cdots \ell_{s}^{c_{s}}\right)=\operatorname{deg} \Delta
$$


We translate the information we have collected about the rings $R \subseteq T$ and $\hat{R} \subseteq \hat{T}$ to information about the rings $\mathcal{O}_{\mathcal{C}, p} \subseteq \overline{\mathcal{O}_{\mathcal{C}, p}}$ and $\widehat{\mathcal{O}_{\mathcal{C}, p}} \subseteq\left(\overline{\mathcal{O}_{\mathcal{C}, p}}\right)^{\wedge}$. Recall first that all four rings are subrings of $\operatorname{Quot}\left(\boldsymbol{k}\left[I_{d}\right]\right)=\operatorname{Quot}\left(\boldsymbol{k}\left[B_{d}\right]\right)$ :

$$
\begin{aligned}
\mathcal{O}_{\mathcal{C}, p} & =\left\{\frac{f}{g} \mid f \in \boldsymbol{k}\left[I_{d}\right] \text { and } g \in \boldsymbol{k}\left[I_{d}\right] \backslash \mathfrak{p} \text { are homogeneous of the same degree }\right\} \\
\overline{\mathcal{O}_{\mathcal{C}, p}} & =\left\{\frac{f}{g} \mid f \in \boldsymbol{k}\left[B_{d}\right] \text { and } g \in \boldsymbol{k}\left[I_{d}\right] \backslash \mathfrak{p} \text { are homogeneous of the same degree }\right\} \\
R & =\boldsymbol{k}\left[I_{d}\right]_{\mathfrak{p}}=\left\{\frac{f}{g} \mid f \in \boldsymbol{k}\left[I_{d}\right] \text { and } g \in \boldsymbol{k}\left[I_{d}\right] \backslash \mathfrak{p}\right\}, \text { and } \\
T & =\boldsymbol{k}\left[B_{d}\right]_{\mathfrak{p}}=\left\{\frac{f}{g} \mid f \in \boldsymbol{k}\left[B_{d}\right] \text { and } g \in \boldsymbol{k}\left[I_{d}\right] \backslash \mathfrak{p}\right\}
\end{aligned}
$$

Observe that $g_{n}^{\prime}$ is a unit of $R$ which is transcendental over $\mathcal{O}_{\mathcal{C}, p}$. It is not difficult to check that the two subrings $\mathcal{O}_{\mathcal{C}, p}\left(g_{n}^{\prime}\right)$, see (0.6), and $R$ of $\operatorname{Quot}\left(\boldsymbol{k}\left[I_{d}\right]\right)$ are equal and therefore, $R$ and $\mathcal{O}_{\mathcal{C}, p}$ have the same multiplicity. The ring $\overline{\mathcal{O}_{\mathcal{C}, p}}$ is a subring of $T$. Dehomogenization and homogenization

$$
\mathfrak{a} \cap \overline{\mathfrak{A}} \overline{\mathcal{O}_{\mathcal{C}, p}} \leftarrow \mathfrak{a} T
$$

provide a one-to-one correspondence between the maximal ideals $\mathfrak{a}$ of $\overline{\mathcal{O}_{\mathcal{C}, p}}$ and the maximal ideals of $\mathfrak{A} \in\left\{\mathfrak{M}_{i} \cap T \mid 1 \leq i \leq v\right\}$ of $T$. Recall that the invariant $s_{\mathcal{C}, p}$ is equal to the number of branches of $\mathcal{C}$ at $p$. Thus,

$$
\begin{aligned}
s_{\mathcal{C}, p} & =\text { the number of minimal prime ideals of } \widehat{\mathcal{O}_{\mathcal{C}, p}} \\
& =\text { the number of maximal prime ideals of } \overline{\mathcal{O}_{\mathcal{C}, p}} \\
& =\text { the number of maximal prime ideals of } T=v
\end{aligned}
$$

Finally, we compare the multiplicities $e\left(\hat{R} / J_{i}\right)$ and $e\left(\widehat{\mathcal{O}_{\mathcal{C}, p}} / \mathcal{J}_{i}\right)$, where $\mathcal{J}_{i}$ is the minimal prime of $\widehat{\mathcal{O}_{\mathcal{C}, p}}$ which corresponds to the minimal prime ideal $J_{i}$ of $\hat{R}$. We have already shown that $e\left(\hat{R} / J_{i}\right)=c_{i}$. We complete the proof of the Lemma by showing that

$$
e\left(\widehat{\mathcal{O}_{\mathcal{C}, p}} / \mathcal{J}_{i}\right)=e\left(\hat{R} / J_{i}\right)
$$

As before, we have $R=\mathcal{O}_{\mathcal{C}, p}\left(g_{n}^{\prime}\right)$. It is well known, and easy to show, that if $z$ is an indeterminate over a local ring $A$, then

$$
\widehat{A(z)}=\widehat{\hat{A}(z)}
$$


where the meaning of $A(z)$ is given in (0.6) and each completion is the completion of a local ring in its maximal ideal adic topology. It follows that

$$
\hat{R}=\left(\widehat{\mathcal{O}_{\mathcal{C}, p}}\left(g_{n}^{\prime}\right)\right)^{\widehat{\imath}} .
$$

The ideal $\mathcal{J}_{i}$ of $\widehat{\mathcal{O}_{\mathcal{C}, p}}$ is $J_{i} \cap \widehat{\mathcal{O}_{\mathcal{C}, p}}$. We first prove

$$
\mathcal{J}_{i} \hat{R}=J_{i}
$$

The ring $\widehat{\mathcal{O}_{\mathcal{C}, p}} / \mathcal{J}_{i}$ is a complete local domain and $g_{n}^{\prime}$ is an indeterminate over this ring; so, Lemma 1.16 yields that $\left(\left(\frac{\widehat{\mathcal{O}_{\mathcal{C}, p}}}{\mathcal{J}_{i}}\right)\left(g_{n}^{\prime}\right)\right)^{\widehat{ }}$ is a domain. On the other hand,

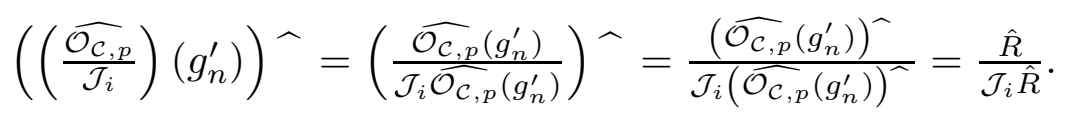

Thus, $\mathcal{J}_{i} \hat{R}$ is a prime ideal of $\hat{R}$ with $\mathcal{J}_{i} \hat{R} \subseteq J_{i}$. The ideal $J_{i}$ is a minimal prime ideal of $\hat{R}$; so (1.24) is established and we have

$$
e\left(\frac{\hat{R}}{J_{i}}\right)=e\left(\frac{\hat{R}}{\mathcal{J}_{i} \hat{R}}\right)=e\left(\left(\left(\frac{\widehat{\mathcal{O}_{\mathcal{C}, p}}}{\mathcal{J}_{i}}\right)\left(g_{n}^{\prime}\right)\right) \hat{)}\right)=e\left(\left(\frac{\widehat{\mathcal{O}_{\mathcal{C}, p}}}{\mathcal{J}_{i}}\right)\left(g_{n}^{\prime}\right)\right)=e\left(\frac{\widehat{\mathcal{O}_{\mathcal{C}}, p}}{\mathcal{J}_{i}}\right)
$$

which is $(1.23)$.

We close this section with the observation that every parameterization of a curve leads to a parameterization of the branches of the curve.

Observation 1.25. Adopt the Data of 1.1, with $\boldsymbol{k}$ algebraically closed. Then there is a one-to-one correspondence between the points of $\mathbb{P}^{1}$ and the branches of $\mathcal{C}$.

Proof. Fix a point $q$ in $\mathbb{P}^{1}$. Let $p$ be the point $\Psi(q)$ on $\mathcal{C}$. Form the ideal $\mathfrak{p}$ of $\boldsymbol{k}\left[I_{d}\right]$ as described in Remark 1.17 (c) and form the rings

$$
R=k\left[I_{d}\right]_{\mathfrak{p}} \subseteq T=k\left[B_{d}\right]_{\mathfrak{p}} \subseteq S=B_{\mathfrak{p}}
$$

as described in Remark 1.17 (d). There are explicit, well-defined, one-to-one correspondences between each of the following sets:

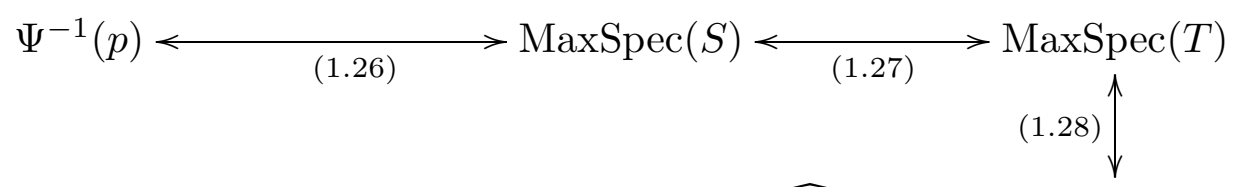

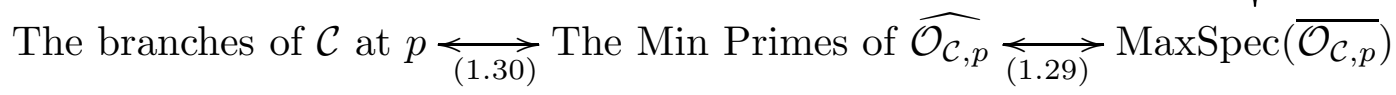


If $q_{1}$ is a point in $\Psi^{-1}(p)$ and $\mathfrak{q}_{1}$ is the homogeneous prime ideal of $B$ which corresponds to $q_{1}$, then the correspondence (1.26) sends $q_{1}$ to $\mathfrak{q}_{1} S$ as shown in (1.19). The correspondence (1.27) is described in Remark 1.17 (e). The correspondence (1.28) is explained in (1.22). Proposition 1.12 accounts for (1.29), and (1.30) is the definition of branch.

Remark 1.31. We say that an ideal of $B=\boldsymbol{k}[x, y]$ is a height one linear ideal if it is generated by one non-zero linear form. There is a one-to-one correspondence between the height one linear ideals of $B$ and the points of $\mathbb{P}^{1}$. Thus, Observation 1.25 gives a one-to-one correspondence between the height one linear ideals of $B$ and the branches of $\mathcal{C}$. If $(\ell)$ is a height one linear ideal of $B$, then let $\mathcal{C}(\ell)$ be the corresponding branch of $\mathcal{C}$. It makes sense to speak about the multiplicity of the branch $\mathcal{C}(\ell)$ because $\ell$ corresponds to a point $q$ in $\mathbb{P}^{1}, \Psi(q)=p$ is a point on $\mathcal{C}, \mathcal{C}(\ell)$

is a minimal prime ideal of $\widehat{\mathcal{O}_{\mathcal{C}}, p}$, and the multiplicity of the local ring $\widehat{\mathcal{O}_{\mathcal{C}, p}} / \mathcal{C}(\ell)$ is called the multiplicity of the branch $\mathcal{C}(\ell)$.

We return to the ideas of Remark 1.31 in Section 7, where we calculate the multiplicity of the branch $\mathcal{C}(\ell)$ in terms of data found in the Jacobian ideal of the parameterization of $\mathcal{C}$.

\section{Section 2. The Triple Lemma.}

The General Lemma in Section 1 shows how the generalized row ideals of the Hilbert-Burch matrix of a parameterization of a rational curve $\mathcal{C}$ encode information about the singularities on $\mathcal{C}$. In the present section we describe how one can read information about the infinitely near singularities of $\mathcal{C}$ from the Hilbert-Burch matrix. Theorem 2.1 deals with infinitely near singularities in the first neighborhood of $\mathcal{C}$. Corollary 2.5 is mainly concerned with infinitely near singularities in the second neighborhood of $\mathcal{C}$. We used our first version of Corollary 2.5 to classify singularities of multiplicity three (triple points) on rational plane curves of degree six and for that reason we call this result the Triple Lemma. We now are able to classify singularities of degree $d / 2$ on rational plane curves of even degree $d$; see Theorem 4.8 .

Adopt Data 1.1 with $n=3$, and $\boldsymbol{k}$ an algebraically closed field. Assume that $p$ is a singular point on the curve $\mathcal{C}$ of multiplicity $d_{j}$, for $j$ equal to 1 or 2 . In Theorem 2.1 we describe how to read the infinitely near singularities in the first neighborhood of $p$ from the Hilbert-Burch matrix $\varphi$ for the parameterization of $\mathcal{C}$. Corollary 1.9 shows how to re-arrange the data so that $p$ becomes the point $[0: 0: 1]$ and $\varphi$ has the form described in Theorem 2.1.

Theorem 2.1. Adopt Data 1.1 with $n=3$, and $\boldsymbol{k}$ an algebraically closed field. Assume that $p=[0: 0: 1]$ is a singular point on the curve $\mathcal{C}$ of multiplicity $d_{j}$, for 
j equal to 1 or 2 . Assume further that

$$
\varphi=\left[\begin{array}{cc}
P_{1} & Q_{1} \\
P_{2} & Q_{2} \\
0 & Q_{3}
\end{array}\right]
$$

where the $P_{i}$ and the $Q_{i}$ are homogeneous forms from $B$ with $\operatorname{deg} P_{i}=d-d_{j}$ and $\operatorname{deg} Q_{i}=d_{j}$. Then, a point $P=(p,[a: b]) \in \mathbb{P}^{2} \times \mathbb{P}^{1}$ lies on the blowup of $\mathcal{C}$ at $p$ if and only if $\operatorname{gcd}\left(Q_{3}, a P_{1}+b P_{2}\right)$ is not a constant. In this case, the multiplicity of $P$ on the blowup is the degree of $\operatorname{gcd}\left(Q_{3}, a P_{1}+b P_{2}\right)$.

Proof. Write $\Delta=P_{1} Q_{2}-P_{2} Q_{1}$. Notice that $\operatorname{gcd}\left(P_{1}, P_{2}\right)=1$ and $\operatorname{gcd}\left(Q_{3}, \Delta\right)=1$ since $I_{2}(\varphi)$ has height 2 . Corollary 1.9 shows how to modify $Q_{1}$ and $Q_{2}$ in order to have $\operatorname{gcd}\left(P_{1}, Q_{1}\right)=1$ and $\operatorname{gcd}\left(P_{2}, Q_{2}\right)=1$. Passing to an affine chart we may assume that $p$ is the origin on the affine curve parametrized by $\left(\frac{g_{1}}{g_{3}}, \frac{g_{2}}{g_{3}}\right)$. The blowup of this curve at the origin has two charts, parametrized by $\left(\frac{g_{1}}{g_{3}}, \frac{g_{2}}{g_{1}}\right)$ and $\left(\frac{g_{2}}{g_{3}}, \frac{g_{1}}{g_{2}}\right)$, respectively. Homogenizing we obtain two curves $\mathcal{C}^{\prime}$ and $\mathcal{C}^{\prime \prime}$ in $\mathbb{P}^{2}$ parametrized by $\left[g_{1}^{2}: g_{2} g_{3}: g_{1} g_{3}\right]$ and $\left[g_{2}^{2}: g_{1} g_{3}: g_{2} g_{3}\right]$, respectively. After dividing by a common factor these parameterizations become

$$
\left[P_{2}^{2} Q_{3}:-P_{1} \Delta: P_{2} \Delta\right] \text { and }\left[P_{1}^{2} Q_{3}: P_{2} \Delta:-P_{1} \Delta\right]
$$

respectively. One easily sees that the entries of either vector generate an ideal of height 2 and the corresponding Hilbert Burch matrices are

$$
\left[\begin{array}{cc}
0 & -\Delta \\
P_{2} & 0 \\
P_{1} & P_{2} Q_{3}
\end{array}\right] \text { and }\left[\begin{array}{cc}
0 & \Delta \\
P_{1} & 0 \\
P_{2} & P_{1} Q_{3}
\end{array}\right] \text {. }
$$

Notice that the exceptional fiber of the blowup consists of the points on $\mathcal{C}^{\prime}$ or $\mathcal{C}^{\prime \prime}$ that are of the form $[0: c: 1]$. Observation 1.4 shows that a point $[0: c: 1]$ lies on $\mathcal{C}^{\prime}$ if and only if $\operatorname{gcd}\left(Q_{3}, P_{1}+c P_{2}\right)$ is not a constant, and that it is on $\mathcal{C}^{\prime \prime}$ if and only if $\operatorname{gcd}\left(Q_{3}, c P_{1}+P_{2}\right)$ is not a constant. Furthermore, Theorem 1.8 shows that the degree of this gcd gives the multiplicity of the point on the blowup.

Corollary 2.3. Adopt Data 1.1 with $n=3$ and $\boldsymbol{k}$ an algebraically closed field. Then the infinitely near singularities of $\mathcal{C}$ have multiplicity at most $d_{1}$.

Proof. It suffices to see this for the multiplicity of the singular points in the first neighborhood of a point $p$ on $\mathcal{C}$. If $m_{p} \leq d_{1}$ then the assertion is clear. Otherwise, Corollary 1.9 shows that $m_{p}=d_{2}$. Notice that in the setting of Theorem 2.1 the form $a P_{1}+b P_{2}$ cannot be zero and hence $\operatorname{deg}\left(\operatorname{gcd}\left(Q_{3}, a P_{1}+P_{2}\right)\right) \leq d_{1}$. Now the present assertion follows from the theorem. 
Remark 2.4. Let $q$ be a root of $Q_{3}$. Notice that the point

$$
P=(p,[a: b])=\left(\Psi(q),\left[-P_{2}(q): P_{1}(q)\right]\right)
$$

is a point in the blowup of $\mathcal{C}$ at $p$ and conversely each point in the blowup of $\mathcal{C}$ at $p$ is obtain in this manner.

Corollary 2.5 is the "Triple Lemma"; it concerns singularities of multiplicity $c=d / 2$, on, or infinitely near, $\mathcal{C}$, where $d$ is the degree of $\mathcal{C}$. We first show that if $q$ is a singularity of multiplicity $c$ infinitely near to $\mathcal{C}$, then $q$ is infinitely near to a singularity $p$ on $\mathcal{C}$ of multiplicity $c$. In particular, it is not possible for $c=m_{q}<m_{p}$. The most significant part of the result is assertion (4), where we study singular points of multiplicity $c$ which are in the second neighborhood of $\mathcal{C}$. A complete description of all multiplicity $c$ singularities which appear on or infinitely near a rational plane curve $\mathcal{C}$ of degree $d=2 c$ appears in Theorem 4.8.

Remark. Assertion (1) in the next result is a purely geometric statement. We wonder if there is a geometric argument for it.

Corollary 2.5. Adopt Data 1.1 with $n=3, k$ an algebraically closed field, and $d$ equal to the even number $2 c$.

(1) If $p$ is a point on $\mathcal{C}$ and $q$ is a singularity of multiplicity $c$ infinitely near to $p$, then the multiplicity of $p$ is also equal to $c$.

(2) If $p$ is a singularity on $\mathcal{C}$ of multiplicity $c$, then the parameters $d_{1}$ and $d_{2}$ are both equal to $c$.

(3) Suppose that $p$ is a singularity on $\mathcal{C}$ of multiplicity $c$. Then there is a singularity of $\mathcal{C}$, infinitely near to $p$, of multiplicity $c$ if and only if there exist invertible matrices $\chi$ and $\xi$, with entries in $\boldsymbol{k}$, such that $(0,0,1)=p \chi$ and

$$
\chi \varphi \xi=\left[\begin{array}{cc}
P_{1} & Q_{1} \\
Q_{3} & Q_{2} \\
0 & Q_{3}
\end{array}\right],
$$

where the homogeneous forms $P_{1}$ and $Q_{i}$ all have degree $c$.

(4) Suppose that $p$ is a singularity on $\mathcal{C}$ of multiplicity $c$. Then there are two singularities of $\mathcal{C}$, infinitely near to $p$, of multiplicity $c$ if and only if there exist invertible matrices $\chi$ and $\xi$, with entries in $\boldsymbol{k}$, such that $(0,0,1)=p \chi$ and

$$
\chi \varphi \xi=\left[\begin{array}{cc}
Q_{2} & Q_{1} \\
Q_{3} & Q_{2} \\
0 & Q_{3}
\end{array}\right],
$$

where the homogeneous forms $Q_{i}$ all have degree $c$. 
Proof. First apply Corollary 2.3. If some infinitely near singularity of $\mathcal{C}$ has multiplicity $c$ or higher, then $c \leq d_{1} \leq(1 / 2) d=c$. Assertions (1) and (2) have been established. Henceforth, we assume that $p$ is a singularity of multiplicity $m_{p}$ on $\mathcal{C}$. Assertion (3) is an immediate consequence of Theorem 2.1. We prove (4). In light of $(3)$, we start with the singularity $p=[0: 0: 1]$ on the curve $\mathcal{C}$ whose Hilbert-Burch matrix is equal to

$$
\varphi^{\prime}=\left[\begin{array}{cc}
P_{1} & Q_{1} \\
Q_{3} & Q_{2} \\
0 & Q_{3}
\end{array}\right],
$$

where the homogeneous forms $P_{1}$ and $Q_{i}$ all have degree $c$ and

$$
\operatorname{gcd}\left(Q_{2}, Q_{3}\right)=\operatorname{gcd}\left(P_{1}, Q_{3}\right)=\operatorname{gcd}\left(P_{1}, Q_{1}\right)=1 .
$$

Let $p^{\prime}$ be the singular point of multiplicity $c$ in the first neighborhood of $p$. Remark 2.4 shows that $p^{\prime}$ is the point $(p,[0: 1])$ on the blowup of $\mathcal{C}$. Thus, in the language of the proof of Theorem 2.1, $p^{\prime}$ is on $\mathcal{C}^{\prime \prime}$. Keep in mind that the polynomial $P_{2}$ of the original Hilbert-Burch matrix $\varphi$ has been replaced by $Q_{3}$ in the Hilbert-Burch matrix $\varphi^{\prime}$ of (2.6) and apply column operations to the matrix on the right side of (2.2), to see that one Hilbert-Burch matrix for the parameterization of $\mathcal{C}^{\prime \prime}$ is

$$
\left[\begin{array}{cc}
\Delta & 0 \\
-P_{1}^{2} & P_{1} \\
0 & Q_{3}
\end{array}\right] .
$$

Let $p^{\prime \prime}=\left(p^{\prime},[a: b]\right) \in \mathbb{P}^{2} \times \mathbb{P}^{1}$ be a point on the blowup of $\mathcal{C}^{\prime \prime}$ at $p^{\prime}$. Theorem 2.1 now shows that $p^{\prime \prime}$ is a singular point of multiplicity $c$ in the first neighborhood of $p^{\prime}$ if and only if

$$
\operatorname{deg} \operatorname{gcd}\left(Q_{3}, a \Delta-b P_{1}^{2}\right)=c .
$$

Recall that $\Delta=P_{1} Q_{2}-Q_{1} Q_{3}, \operatorname{gcd}\left(P_{1}, Q_{3}\right)=1$, and $Q_{2}, Q_{3}$, and $P_{1}$ all are homogeneous forms of degree $c$. It follows that $p^{\prime \prime}$ is a singular point of multiplicity $c$ in the first neighborhood of $p^{\prime}$ if and only if

$$
\gamma Q_{3}=a Q_{2}-b P_{1}
$$

for some constant $\gamma \in \boldsymbol{k}$. The equation (2.7) cannot hold if $b=0$, since $[a: b]$ is a point in $\mathbb{P}^{1}$ and $Q_{2}$ and $Q_{3}$ are relatively prime. Therefore,

there exists a singular point of multiplicity $c$ in the first neighborhood of $p^{\prime}$

$\Longleftrightarrow P_{1}$ is in the vector space generated by $Q_{2}$ and $Q_{3}$

$\Longleftrightarrow$ there exist invertible matrices $\chi^{\prime}$ and $\xi^{\prime}$ so that $\chi \varphi^{\prime} \xi$ has the form of the matrix of (4). 


\section{Section 3. The BiProj Lemma.}

Let $\varphi$ be a Hilbert-Burch matrix which corresponds to a parameterized plane curve $\mathcal{C}$ of degree $d$. Points of $\mathbb{P}^{2}$ give rise to generalized row ideals of the matrix $\varphi$. Thus, features of the generalized row ideals reflect properties of the corresponding points. For example, a generalized row ideal of $\varphi$ encodes information that can be used to determine if the corresponding point $p$ is on $\mathcal{C}$ and, if so, what type of singularity occurs at $p$.

In this section, we focus on the situation where the degree of $\mathcal{C}$ is even ( $\operatorname{so} d=2 c)$ and we describe the singular points on, or infinitely near, $\mathcal{C}$ of multiplicity $c$. We know from Corollary 2.5 that such a point exists if and only if all entries of $\varphi$ have the same degree $c$ and the corresponding generalized row has a generalized zero. This leads us to consider column operations on $\varphi$, which we identify with points in $\mathbb{P}^{1}$. Thus, inside $\mathbb{P}^{2} \times \mathbb{P}^{1}$ we consider the closed subset consisting of pairs

$$
\text { (row operation, column operation) }
$$

that lead to a generalized zero of $\varphi$. Projection onto the first factor gives the singular points of multiplicity $c$. On the other hand, projection onto the second factor yields a finite set of points in $\mathbb{P}^{1}$ that is easier to study yet reflects properties of the set of singular points of multiplicity $c$ on the plane curve $\mathcal{C}$.

Fix a Hilbert-Burch matrix $\varphi$ in which every entry is a homogeneous form of degree $c$. To find a singularity of multiplicity $\mathrm{c}$ on $\mathcal{C}$ we need to describe a generalized zero of $\varphi$. In other words, we look for $(p, q)$ in $\mathbb{P}^{2} \times \mathbb{P}^{1}$ such that $p \varphi q^{\mathrm{T}}=0$. Consider the polynomial $\boldsymbol{T} \varphi \boldsymbol{u}^{\mathrm{T}} \in \boldsymbol{k}[\boldsymbol{T}, \boldsymbol{u}, x, y]$, where $\boldsymbol{T}=\left[T_{1}, T_{2}, T_{3}\right]$ and $\boldsymbol{u}=\left[u_{1}, u_{2}\right]$ are matrices of indeterminates. We extract the variables $x$ and $y$ from the critical polynomial $\boldsymbol{T} \varphi \boldsymbol{u}^{\mathrm{T}}$. For each positive integer $i$, let $\rho^{(i)}$ be the following $1 \times(i+1)$ row vector of all monomials of degree $i$ in $k[x, y]$ :

$$
\rho^{(i)}=\left[y^{i}, x y^{i-1}, x^{2} y^{i-2}, \ldots, x^{i-1} y, x^{i}\right] .
$$

Define $C$ and $A$ to be the matrices with

$$
\boldsymbol{T} \varphi=\rho^{(c)} C \text { and } \quad C \boldsymbol{u}^{\mathrm{T}}=A \boldsymbol{T}^{\mathrm{T}},
$$

so that the entries of $C$ are linear forms in $k[T]$ and the entries of $A$ are linear forms in $\boldsymbol{k}[\boldsymbol{u}]$. One now has

$$
\boldsymbol{T} \varphi \boldsymbol{u}^{\mathrm{T}}=\rho^{(c)} C \boldsymbol{u}^{\mathrm{T}}=\rho^{(c)} A \boldsymbol{T}^{\mathrm{T}}
$$

Thus,

the set of $(p, q)$ in $\mathbb{P}^{2} \times \mathbb{P}^{1}$ such that $p \varphi q^{\mathrm{T}}=0$ is the zero set, in $\mathbb{P}^{2} \times \mathbb{P}^{1}$, of the bihomogeneous ideal $I_{1}\left(C \boldsymbol{u}^{\mathrm{T}}\right)=I_{1}\left(A T^{\mathrm{T}}\right)$. 
In Theorem 3.14, we obtain two isomorphisms of schemes

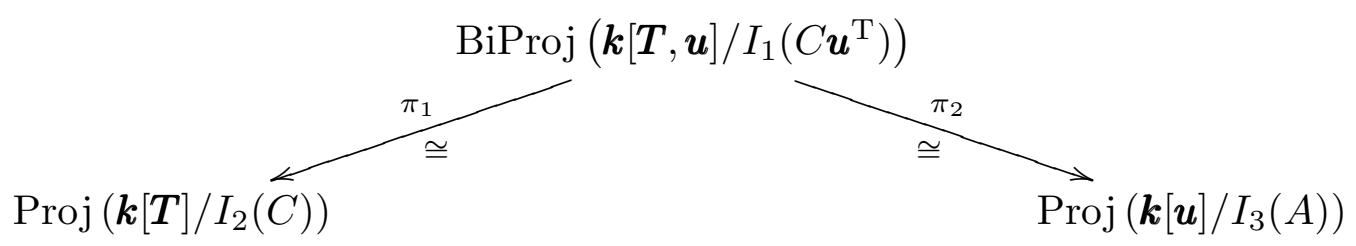

and we exploit these isomorphisms in Theorem 3.22 to describe the singularities on $\mathcal{C}$ of multiplicity $c$. The equation

$$
C \boldsymbol{u}^{\mathrm{T}}=A \boldsymbol{T}^{\mathrm{T}}
$$

provides symmetry. Theorem 3.5 is used twice to produce the isomorphisms of (3.3)

Theorem 3.5. Let $S=\boldsymbol{k}\left[x_{1}, \ldots, x_{m}, y_{1}, \ldots, y_{n}\right]$ be a bi-graded polynomial ring with $\operatorname{deg} x_{i}=(1,0)$ and $\operatorname{deg} y_{i}=(0,1)$, and $R$ be the sub-algebra $\boldsymbol{k}\left[x_{1}, \ldots, x_{m}\right]$ of $S$. Let $J$ be an $S$-ideal generated by bi-homogeneous forms which are linear in the $y$ 's. Write $J=I_{1}(\phi \boldsymbol{y})$ where $\boldsymbol{y}=\left[y_{1}, \ldots, y_{n}\right]^{\mathrm{T}}$ and $\phi$ is a matrix with entries in $R$. The entries in each row of $\phi$ are homogeneous of the same degree. Consider the natural projection map $\pi: \operatorname{BiProj}(S / J) \rightarrow \operatorname{Proj}(R)$. If the ideal $I_{n-1}(\phi)$ is zero-dimensional in $R$, then $\pi$ is an isomorphism onto its image and this image is defined scheme-theoretically by the $R$-ideal $I_{n}(\phi)$.

Notice that $\operatorname{im} \pi=\operatorname{Proj}\left(R /\left(J:\left(I_{1}(\boldsymbol{x}) I_{1}(\boldsymbol{y})\right)^{\infty}\right) \cap R\right) \subseteq \operatorname{Proj}(R)$. The theorem means that $\pi$ gives a bijection $\operatorname{BiProj}(S / J) \rightarrow \operatorname{Proj}\left(R / I_{n}(\phi)\right)$ which induces isomorphisms at the level of local rings.

Proof. We prove the result after localizing at any fixed one-dimensional homogeneous prime ideal $\mathfrak{m}$ of $R$. The ideal $I_{n-1}(\phi) R_{\mathfrak{m}}$ is the unit ideal by hypothesis; therefore, there are invertible matrices $U$ and $V$ with

$$
U \phi V=\left[\begin{array}{cc}
I_{n-1} & 0 \\
0 & \phi^{\prime}
\end{array}\right]
$$

for some column vector $\phi^{\prime}$ with entries in $R_{\mathfrak{m}}$. Write $S_{\mathfrak{m}}$ to mean $(R \backslash \mathfrak{m})^{-1} S$. We have $S_{\mathfrak{m}}=R_{\mathfrak{m}}\left[y_{1}^{\prime}, \ldots, y_{n}^{\prime}\right]$, where $\boldsymbol{y}^{\prime}=V^{-1} \boldsymbol{y}$. The ideal $J S_{\mathfrak{m}}$ is equal to $I_{1}(\phi \boldsymbol{y}) S_{\mathfrak{m}}=\left(y_{1}^{\prime}, \ldots, y_{n-1}^{\prime}, I_{1}\left(\phi^{\prime}\right) y_{n}^{\prime}\right)$.

The ring map $S / J \rightarrow S_{\mathfrak{m}} / J S_{\mathfrak{m}}$ induces the inclusion

$$
\operatorname{Proj}\left(S_{\mathfrak{m}} / J S_{\mathfrak{m}}\right) \subseteq \operatorname{BiProj}(S / J)
$$


and the natural projection map $\pi: \operatorname{BiProj}(S / J) \rightarrow \operatorname{Proj}(R)$ restricts to become

$$
\operatorname{Proj}\left(S_{\mathfrak{m}} / J S_{\mathfrak{m}}\right) \stackrel{\pi \mid}{\longrightarrow} \operatorname{Spec}\left(R_{\mathfrak{m}}\right) .
$$

The image of $\pi \mid$ is the subscheme $\operatorname{Spec}\left(\frac{R_{\mathfrak{m}}}{\left(J S_{\mathfrak{m}}: I_{1}(\boldsymbol{y})^{\infty}\right) \cap R_{\mathfrak{m}}}\right)$ of $\operatorname{Proj}(R)$. We must show that

(1) $\pi \mid$ is an isomorphism onto its image, and

(2) the image of $\pi \mid$ is defined scheme-theoretically by the ideal $I_{n}(\phi) R_{\mathfrak{m}}$.

Notice that

$$
\begin{aligned}
& J S_{\mathfrak{m}}: I_{1}(\boldsymbol{y})^{\infty}=\left(y_{1}^{\prime}, \ldots, y_{n-1}^{\prime}, I_{1}\left(\phi^{\prime}\right) y_{n}^{\prime}\right) S_{\mathfrak{m}}:\left(y_{1}^{\prime}, \ldots, y_{n-1}^{\prime}, y_{n}^{\prime}\right)^{\infty} \\
& =\left(y_{1}^{\prime}, \ldots, y_{n-1}^{\prime}, I_{1}\left(\phi^{\prime}\right) y_{n}^{\prime}\right) S_{\mathfrak{m}}:\left(y_{n}^{\prime}\right)^{\infty}=\left(y_{1}^{\prime}, \ldots, y_{n-1}^{\prime}, I_{1}\left(\phi^{\prime}\right)\right) S_{\mathfrak{m}}
\end{aligned}
$$

Therefore, the image of $\pi \mid$ is defined scheme-theoretically by

$$
\left(J S_{\mathfrak{m}}: I_{1}(\boldsymbol{y})^{\infty}\right) \cap R_{\mathfrak{m}}=I_{1}\left(\phi^{\prime}\right) R_{\mathfrak{m}}=I_{n}(\phi) R_{\mathfrak{m}}
$$

which proves the second assertion. To show the first claim, notice that the natural map

$$
R_{\mathfrak{m}} \rightarrow \frac{R_{\mathfrak{m}}\left[y_{n}^{\prime}\right]}{I_{1}\left(\phi^{\prime}\right) y_{n}^{\prime} R_{\mathfrak{m}}\left[y_{n}^{\prime}\right]}=\frac{S_{\mathfrak{m}}}{J S_{\mathfrak{m}}}
$$

induces $\pi \mid: \operatorname{Proj}\left(S_{\mathfrak{m}} / J S_{\mathfrak{m}}\right) \rightarrow \operatorname{Spec}\left(R_{\mathfrak{m}}\right) ;$ furthermore,

$$
\begin{aligned}
& \operatorname{Proj}\left(\frac{S_{\mathfrak{m}}}{J S_{\mathfrak{m}}}\right)=\operatorname{Proj}\left(\frac{R_{\mathfrak{m}}\left[y_{n}^{\prime}\right]}{I_{1}\left(\phi^{\prime}\right) y_{n}^{\prime} R_{\mathfrak{m}}\left[y_{n}^{\prime}\right]}\right)=\operatorname{Proj}\left(\frac{R_{\mathfrak{m}}\left[y_{n}^{\prime}\right]}{I_{1}\left(\phi^{\prime}\right) y_{n}^{\prime} R_{\mathfrak{m}}\left[y_{n}^{\prime}\right]:\left(y_{n}^{\prime}\right)^{\infty}}\right) \\
& =\operatorname{Proj}\left(\frac{R_{\mathfrak{m}}\left[y_{n}^{\prime}\right]}{I_{1}\left(\phi^{\prime}\right) R_{\mathfrak{m}}\left[y_{n}^{\prime}\right]}\right)=\operatorname{Proj}\left(\frac{R_{\mathfrak{m}}}{I_{1}\left(\phi^{\prime}\right) R_{\mathfrak{m}}}\left[y_{n}^{\prime}\right]\right)=\operatorname{Spec}\left(\frac{R_{\mathfrak{m}}}{I_{1}\left(\phi^{\prime}\right) R_{\mathfrak{m}}}\right) \\
& =\operatorname{Spec}\left(\frac{R_{\mathfrak{m}}}{I_{n}(\phi) R_{\mathfrak{m}}}\right) \cdot
\end{aligned}
$$

The next Lemma analyzes a general version of (3.4). This Lemma is a step toward verifying that the hypothesis of Theorem 3.5 are satisfied twice in (3.3). It is fortuitous that the information about the minors of $A$ translates into information about the columns of $C$ and vice versa. In this result $C$ is a matrix with $s$ columns and $A$ is a matrix with entries from $k\left[u_{1}, \ldots, u_{s}\right]$.

Remark 3.6. If $\lambda \in \mathbb{A}^{s}$, then we abbreviate the generalized column $C \lambda^{\mathrm{T}}$ of $C$ as $C_{\lambda}$; also, $A(\lambda)$ is the matrix of constants which is obtained by evaluating each entry of $A$ at the point $\lambda$. 
Lemma 3.7. Let $\boldsymbol{u}=\left[u_{1}, \ldots, u_{s}\right]$ and $\boldsymbol{T}=\left[T_{1}, \ldots, T_{t}\right]$ be matrices of indeterminates over an algebraically closed field $\boldsymbol{k}, C$ be an $n \times s$ matrix of linear forms from $\boldsymbol{k}[\boldsymbol{T}]$, and $A$ be an $n \times t$ matrix of linear forms from $\boldsymbol{k}[\boldsymbol{u}]$. If $C \boldsymbol{u}^{\mathrm{T}}=A \boldsymbol{T}^{\mathrm{T}}$, then the following statements hold:

$$
\begin{aligned}
I_{i}(A) \neq 0 & \Longleftrightarrow \text { ht } I_{1}\left(C_{\lambda}\right) \geq i \text { for general } \lambda \in \mathbb{A}^{s} \text {, and } \\
\text { ht } I_{i}(A) \geq s & \Longleftrightarrow \text { ht } I_{1}\left(C_{\lambda}\right) \geq i \text { for all non-zero } \lambda \in \mathbb{A}^{s} \text {. }
\end{aligned}
$$

Proof. We have $C_{\lambda}=C \lambda^{\mathrm{T}}=A(\lambda) \boldsymbol{T}^{\mathrm{T}}$. The right hand side of (3.8) holds if and only if

$\operatorname{rank} A(\lambda) \geq i$ for general $\lambda \in \mathbb{A}^{s}$

$$
\Longleftrightarrow I_{i}(A(\lambda)) \neq 0 \text { for general } \lambda \in \mathbb{A}^{s} \Longleftrightarrow I_{i}(A) \neq 0 \text {. }
$$

The right side of (3.9) holds if and only if

$$
\begin{aligned}
& \operatorname{rank} A(\lambda) \geq i \text { for all non-zero } \lambda \in \mathbb{A}^{s} \\
\Longleftrightarrow & I_{i}(A(\lambda)) \neq 0 \text { for all non-zero } \lambda \in \mathbb{A}^{s} \Longleftrightarrow \text { ht } I_{i}(A) \geq s .
\end{aligned}
$$

The last equivalence is due to Hilbert's Nullstellensatz which applies since $k$ is algebraically closed.

Data 3.10. Adopt Data 1.1 with $n=3, d=2 c, d_{1}=d_{2}=c$, and $\boldsymbol{k}$ an algebraically closed field. Let $\boldsymbol{T}$ and $\boldsymbol{u}$ be the row vectors $\boldsymbol{T}=\left[T_{1}, T_{2}, T_{3}\right]$ and $\boldsymbol{u}=\left[u_{1}, u_{2}\right]$ of indeterminates. Recall the row vector $\rho^{(c)}$ from (3.1). Define the matrices $C$ and $A$ by

$$
\begin{aligned}
\boldsymbol{T} \varphi & =\rho^{(c)} C \text { and } \\
C \boldsymbol{u}^{\mathrm{T}} & =A \boldsymbol{T}^{\mathrm{T}},
\end{aligned}
$$

so that the entries of $C$ are linear forms in $\boldsymbol{k}[\boldsymbol{T}]$ and the entries entries of $A$ are linear forms in $\boldsymbol{k}[\boldsymbol{u}]$.

Lemma 3.13. Adopt Data 3.10. The following statements hold:

(1) ht $I_{1}(C)=3$,

(2) ht $I_{1}\left(C_{\lambda}\right) \geq 2$ for all non-zero $\lambda \in \mathbb{A}^{2}$, and

(3) ht $I_{2}(A)=2$. 
Proof. (1) The height of $I_{1}(C)$ is three otherwise, since the entries of $C$ are linear, $I_{1}(C)$ is contained in an ideal generated by two linear forms. Equation (3.11) shows that, after row operations, $\varphi$ has a row of zeros yielding $h t(I)=1$. Now we prove (2). Equation (3.11) gives $\rho^{(c)} C_{\lambda}=\boldsymbol{T} \varphi_{\lambda}$. If ht $\left(I_{1}\left(C_{\lambda}\right)\right) \leq 1$, then $\mu\left(I_{1}\left(C_{\lambda}\right)\right) \leq 1$ because the entries of $C$ are linear. Thus, $\left.\mu\left(\boldsymbol{T} \varphi_{\lambda}\right)\right) \leq 1$, which shows that after row operations $\varphi_{\lambda}$ has at most one non-zero entry. This would imply that ht $(I) \leq 1$. Assertion (3) follows from (2) and Lemma 3.7.

Theorem 3.14. Adopt Data 3.10. The following statements hold.

(1) The projections

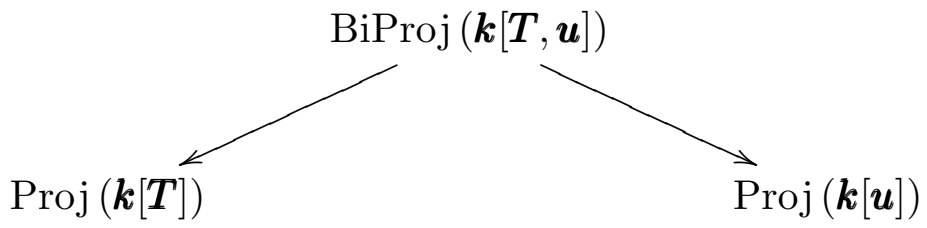

induce isomorphisms

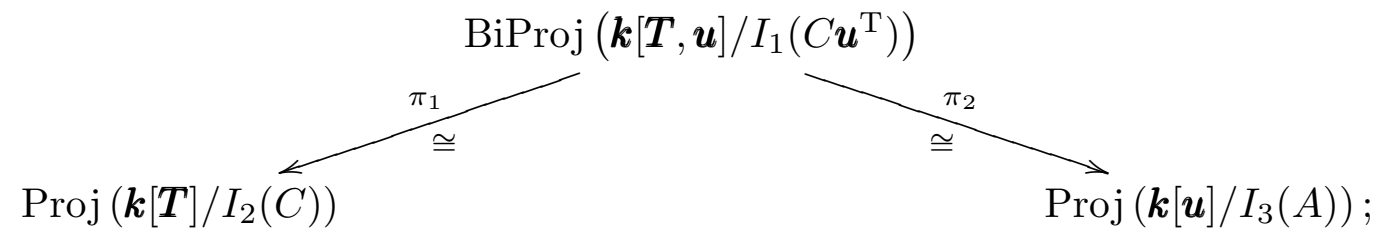

in particular, the schemes $\operatorname{Proj}\left(\frac{\boldsymbol{k}[\boldsymbol{T}]}{I_{2}(C)}\right)$ and $\operatorname{Proj}\left(\frac{\boldsymbol{k}[\boldsymbol{u}]}{I_{3}(A)}\right)$ are isomorphic.

(2) As a subset of $\mathbb{P}^{2}, \operatorname{Proj}\left(\frac{\boldsymbol{k}[\boldsymbol{T}]}{I_{2}(C)}\right)$ is equal to $\left\{p \in \mathcal{C} \mid m_{p}=c\right\}$.

Proof. We apply Theorem 3.5 twice. Each time $S=\boldsymbol{k}[\boldsymbol{T}, \boldsymbol{u}]$ and $J=I_{1}\left(C \boldsymbol{u}^{\mathrm{T}}\right)=$ $I_{1}\left(A \boldsymbol{T}^{\mathrm{T}}\right)$. In the first application $R=\boldsymbol{k}[\boldsymbol{T}]$. Lemma 3.13 ensures that $I_{1}(C)$ is zero-dimensional in $\boldsymbol{k}[\boldsymbol{T}]$. In the second application $R=\boldsymbol{k}[\boldsymbol{u}]$. Again Lemma 3.13 ensures that $I_{2}(A)$ is zero-dimensional in $\boldsymbol{k}[\boldsymbol{u}]$. Assertion (1) is established.

Return to the first setting. Theorem 3.5 also yields that the image of the map

$$
\mathbb{P}^{2} \times \mathbb{P}^{1} \supseteq \operatorname{BiProj}(S / J) \stackrel{\pi}{\rightarrow} \operatorname{Proj}(\boldsymbol{k}[\boldsymbol{T}])
$$

is $\operatorname{Proj}\left(\boldsymbol{k}[\boldsymbol{T}] / I_{2}(C)\right) \subseteq \operatorname{Proj}(\boldsymbol{k}[\boldsymbol{T}])$. On the other hand, as a set

$$
\begin{aligned}
\operatorname{im} \pi & =\left\{p \in \mathbb{P}^{2} \mid \exists q \in \mathbb{P}^{1} \text { with }(p, q) \in V(J)\right\} \\
& =\left\{p \in \mathbb{P}^{2} \mid \exists q \in \mathbb{P}^{1} \text { with } p \varphi q^{\mathrm{T}}=0\right\} \\
& =\left\{p \in \mathcal{C} \mid m_{p}=c\right\} .
\end{aligned}
$$


The equality (3.15) is explained in (3.2) and the equality (3.16) is established in Corollary 1.9.

Theorem 3.14 produces a morphism (even an isomorphism)

$$
\pi_{2} \circ \pi_{1}^{-1}: \operatorname{Proj}\left(\boldsymbol{k}[\boldsymbol{T}] / I_{2}(C)\right) \rightarrow \operatorname{Proj}\left(\boldsymbol{k}[\boldsymbol{u}] / I_{3}(A)\right)
$$

the graph of this morphism is $\operatorname{BiProj}\left(\boldsymbol{k}[\boldsymbol{u}, \boldsymbol{T}] / I_{1}\left(C \boldsymbol{u}^{\mathrm{T}}\right)\right)$, as can be seen from diagram (3.3).

Remark 3.17. The theorem also says, in particular, that if $p$ and $q$ are non-zero row vectors in $\boldsymbol{k}^{3}$ and $\boldsymbol{k}^{2}$, respectively, with $p \varphi q^{\mathrm{T}}=0$, then, up to multiplication by non-zero scalars, $q$ is determined by $p$ and vice versa.

We next isolate one quick result about how the Data of 3.10 can be used to determine the configuration of multiplicity $c$ singularities on a curve $\mathcal{C}$. The complete story is contained in Theorem 3.22.

Corollary 3.18. Adopt Data 3.10. The curve $\mathcal{C}$ has only singularities of multiplicity at most $c-1$ if and only if every generalized column ideal of $C$ has height three.

Proof. All singularities of $\mathcal{C}$ have multiplicity at most $c-1$ if and only if $\mathcal{C}$ has no singularity of multiplicity $c$ by Corollary 1.9. This in turn means ht $I_{3}(A)=2$ according to Theorem 3.14. Finally, by Lemma 3.7, ht $I_{3}(A)=2$ if and only if every generalized column ideal of $C$ has height three.

Corollary 3.19. Adopt Data 3.10 with $c \geq 2$. The following statements hold:

(1) ht $I_{2}(C) \geq 2$,

(2) ht $I_{3}(A) \geq 1$, and

(3) ht $I_{1}\left(C_{\lambda}\right)=3$ for general $\lambda \in \mathbb{A}^{2}$.

Proof. Theorem 3.14 shows that $\operatorname{Proj}\left(\boldsymbol{k}[\boldsymbol{T}] / I_{2}(C)\right)$ is either empty or is a finite set; therefore, (1) and (2) hold. Assertion (3) follows from (2) and Lemma 3.7.

Lemma 3.20. Let $C$ be matrix of linear forms from a polynomial ring $R$ in three variables over a field $\boldsymbol{k}$. If $C$ has 2 columns, some generalized column ideal of $C$ is a zero-dimensional ideal of $R$, and ht $\left(I_{2}(C)\right)=2$, then

$$
e\left(R / I_{2}(C)\right)+\mu\left(I_{2}(C)\right)=6
$$

Proof. Let $R=k\left[T_{1}, T_{2}, T_{3}\right]$. Since $I_{2}(C)$ is an ideal generated by quadrics in a polynomial in three variables, $\mu\left(I_{2}(C)\right) \leq 5$; for otherwise, $I_{2}(C)=\left(T_{1}, T_{2}, T_{3}\right)^{2}$, which would contradict the hypothesis that ht $I_{2}(C)=2$. 
We are allowed to apply row and column operations, to perform linear changes of variables, and to suppress zero rows. Using these operations, we transform $C$ into one of the following four forms:

$$
M_{1}=\left[\begin{array}{cc}
T_{1} & * \\
T_{2} & * \\
T_{3} & *
\end{array}\right], \quad M_{2}=\left[\begin{array}{cc}
T_{1} & f_{1} \\
T_{2} & f_{2} \\
T_{3} & f_{3} \\
0 & T_{1}
\end{array}\right], \quad M_{3}=\left[\begin{array}{cc}
T_{1} & g_{1} \\
T_{2} & g_{2} \\
T_{3} & g_{3} \\
0 & T_{1} \\
0 & T_{2}
\end{array}\right], \quad M_{4}=\left[\begin{array}{cc}
T_{1} & 0 \\
T_{2} & 0 \\
T_{3} & 0 \\
0 & T_{1} \\
0 & T_{2} \\
0 & T_{3}
\end{array}\right]
$$

where the $f_{i}$ are linear forms in $\boldsymbol{k}\left[T_{2}, T_{3}\right]$ and the $g_{i}$ are linear forms in $R=\boldsymbol{k}\left[T_{3}\right]$.

In the case $C=M_{1}$, the ideal $I_{2}(C)$ is a perfect ideal of height 2 with a 2-linear resolution; hence $e\left(R / I_{2}(C)\right)=3=\mu\left(I_{2}(C)\right)$.

In the case $C=M_{2}$, we have $I_{2}(C)=T_{1}\left(T_{1}, T_{2}, T_{3}\right)+J R$, where $J$ is a non-zero ideal of $\boldsymbol{k}\left[T_{2}, T_{3}\right]$ generated by quadrics. Write $\Delta=\operatorname{gcd}(J) \in \boldsymbol{k}\left[T_{2}, T_{3}\right]$. Notice that $T_{1}$ is in $I_{2}(C)^{\mathrm{unm}}$, the unmixed part of $I_{2}(C)$. Therefore, $I_{2}(C)^{\mathrm{unm}}=\left(T_{1}, J\right)^{\mathrm{unm}}=$ $\left(T_{1}, \Delta\right)$, and $e\left(R / I_{2}(C)\right)=e\left(R / I_{2}(C)^{\mathrm{unm}}\right)=\operatorname{deg} \Delta \leq 2$. Observe that $\operatorname{deg} \Delta=2$ if and only if $\mu(J)=1$; thus, $e\left(R / I_{2}(C)\right)=2$ if and only if $\mu\left(I_{2}(C)\right)=4$. It follows that $e\left(R / I_{2}(C)\right)=1$ if and only if $\mu\left(I_{2}(C)\right) \geq 5$, which means that $\mu\left(I_{2}(C)\right)=5$.

In the case $C=M_{3}$, we have $\left(T_{1}, T_{2}\right)\left(T_{1}, T_{2}, T_{3}\right) \subseteq I_{2}(C)$ and these two ideals are equal because $I_{2}(C)$ is generated by at most 5 quadrics. Therefore, $\mu\left(I_{2}(C)\right)=5$ and $e\left(R / I_{2}(C)\right)=e\left(R /\left(T_{1}, T_{2}\right)\right)=1$.

Finally, if $C=M_{4}$, then $\left(T_{1}, T_{2}, T_{3}\right)^{2}=I_{2}(C)$, which is impossible since $I_{2}(C)$ has height two.

Before proceeding, we describe the effect on Data 3.10 of applying row and column operations to $\varphi$.

Remark 3.21. If we replace $\varphi$ by $\varphi^{\prime}=\chi \varphi$ for some invertible matrix $\chi$ with entries in $k$, then the matrices $C$ and $A$ of Data 3.10 become $C^{\prime}=C(\boldsymbol{T} \chi)$ and $A^{\prime}=$ $A \chi^{\mathrm{T}}$. Recall that changing $\varphi$ by $\varphi^{\prime}$ amounts to applying to the curve $\mathcal{C}$ the linear automorphism of $\mathbb{P}^{2}$ defined by the matrix $\chi^{-1}$; see Remark 0.9 . If we replace $\varphi$ by $\varphi^{\prime}=\varphi \xi$ for some invertible matrix $\xi$ with entries in $k$, then the matrices $C$ and $A$ become $C^{\prime}=C \xi$ and $A^{\prime}=A\left(\boldsymbol{u} \xi^{\mathrm{T}}\right)$, for $A(\lambda)$ as defined in Remark 3.6. Notice that column operations on $\varphi$ have no effect on the $g$ 's or on the curve $\mathcal{C}$.

Ultimately, in Corollary 3.23, we use the Jacobian ideal of $\boldsymbol{k}[\boldsymbol{u}] / I_{3}(A)$ over $\boldsymbol{k}$ to count the number of infinitely near singularities of multiplicity $c$. Section 7 contains much information about this object. In the mean time, we recall that if $D$ is an algebra essentially of finite type over a Noetherian ring $L$, then the module of Kähler differentials of $D$ over $L$ is denoted by $\Omega_{D / L}$. The zeroth Fitting ideal of the $D$ module $\Omega_{D / L}$ is called the Jacobian ideal of $D$ over $L$ and is denoted by $\operatorname{Jac}(D / L)$. 
The Jacobian ideal $\operatorname{Jac}(D / L)$ is an ideal of $D$. These objects may be computed using any presentation of the $L$-algebra $D$ and their formation is compatible with localization. Notice that the subsets $\operatorname{Supp}\left(\Omega_{D / L}\right)$ and $V(\operatorname{Jac}(D / L))$ of Spec $D$ are equal. In Section 7 we will use the fact that this common subset of $\operatorname{Spec}(D)$ is the ramification locus of $D$ over $L$.

In [24] we studied Rees algebras of ideals generated by 3 forms of degree 6 in $\boldsymbol{k}[x, y]$. We found that there are exactly seven families of Rees algebras for such ideals. Each member of a given family has the same graded Betti numbers. Four of these family arise when every entry in the syzygy matrix has degree 3 . The distinguishing invariant turned out to be $\mu\left(I_{2}(C)\right.$ ). Ad hoc methods led to this classification. We are very pleased to now see in Theorem 3.22 that this invariant arises naturally and reflects the geometry of the curve.

Theorem 3.22. Adopt Data 3.10.

(1) The ideal $I_{2}(C)$ is zero-dimensional if and only if $I_{3}(A)$ is zero-dimensional if and only if $\operatorname{gcd} I_{3}(A)$ is a unit; otherwise

$$
e\left(\boldsymbol{k}[\boldsymbol{T}] / I_{2}(C)\right)=e\left(\boldsymbol{k}[\boldsymbol{u}] / I_{3}(A)\right)=\operatorname{deg} \operatorname{gcd} I_{3}(A)=6-\mu\left(I_{2}(C)\right) .
$$

(2) The non-associate linear factors of gcd $I_{3}(A)$ correspond to the distinct singular points on $\mathcal{C}$ of multiplicity $c$.

(3) Write $\operatorname{gcd}\left(I_{3}(A)\right)=\prod \ell_{i}^{e_{i}}$, where the $\ell_{i}$ are non-associate linear factors and $e_{i} \geq 1$. Then $e_{i}-1$ is the number of singular points of multiplicity $c$ infinitely near to the point on $\mathcal{C}$ corresponding to $\ell_{i}$.

(4) The degree of $\operatorname{gcd} I_{3}(A)$ is equal to the number of distinct singular points of multiplicity $c$ that are either on $\mathcal{C}$ or infinitely near to $\mathcal{C}$.

Before proving Theorem 3.22, we reformulate part of it in the exact form we will use in Section 6 where we decompose the space of parameterizations of rational plane curves of even degree $d=2 c$ into strata which reflect the configuration of multiplicity $c$ singularities on or infinitely near the corresponding curve.

Corollary 3.23. Retain the notation of Theorem 3.22 and let $D=\boldsymbol{k}[\boldsymbol{u}] / I_{3}(A)$. For assertions (3b) and (3c) assume that char $\boldsymbol{k}$ is not equal to 2 or 3 . The following assertions hold.

(1) The dimension of $D$ is either zero or one.

(2) There are no singularities of multiplicity $c$ on or infinitely near $\mathcal{C}$ if and only if $\operatorname{dim} D=0$.

(3) If $D$ has dimension one, then

(a) $e(D)$ is the number of distinct singular points of multiplicity c that are either on $\mathcal{C}$ or infinitely near to $\mathcal{C}$, 
(b) if $D / \operatorname{Jac}(D / k)$ is a ring of dimension zero then all of the multiplicity $c$ singularities on or infinitely near $\mathcal{C}$ are actually on $\mathcal{C}$, and

(c) if $D / \operatorname{Jac}(D / \boldsymbol{k})$ is a ring of dimension one, $e(D)-e(D / \operatorname{Jac}(D / \boldsymbol{k}))$ is equal to the number of distinct singular points of multiplicity $c$ that are on $\mathcal{C}$.

Proof of Corollary 3.23. Assertion (2) follows from items (2) of Theorem 3.22 and (1) of Corollary 2.5. Now we prove (3b) and (3c). Observe that $\operatorname{deg} \operatorname{gcd} I_{3}(A) \leq 3$; hence each $e_{i} \leq 3$ and $e_{i} \neq \operatorname{char} k$. If $\operatorname{dim} D=1$, then the minimal primes of $D$ are $\ell_{i} D$. Notice that $D_{\ell_{i} D} \cong \boldsymbol{k}[\boldsymbol{u}]_{\ell_{i} \boldsymbol{k}[\boldsymbol{u}]} /\left(\ell_{i}^{e_{i}}\right)$. Hence,

$$
\operatorname{Jac}(D / \boldsymbol{k})_{\ell_{i} D}=\operatorname{Jac}\left(D_{\ell_{i}} / \boldsymbol{k}\right)=\ell_{i}^{e_{i}-1} D_{\ell_{i} D} .
$$

It follows that

$$
\begin{aligned}
\operatorname{dim} D / \operatorname{Jac}(D / \boldsymbol{k})=0 & \Longleftrightarrow \operatorname{Jac}(D / \boldsymbol{k})=D \\
& \Longleftrightarrow \text { the roots of } \operatorname{gcd} I_{3}(A) \text { are distinct }
\end{aligned}
$$

and

$$
e(D / \operatorname{Jac}(D / \boldsymbol{k}))=\sum\left(e_{i}-1\right)
$$

and this is equal to the degree of $\operatorname{deg} \operatorname{gcd} I_{3}(A)$ minus the number of distinct linear factors of $\operatorname{gcd} I_{3}(A)$.

Proof of Theorem 3.22. The schemes $\operatorname{Proj}\left(\boldsymbol{k}[\boldsymbol{T}] / I_{2}(C)\right)$ and $\operatorname{Proj}\left(\boldsymbol{k}[\boldsymbol{u}] / I_{3}(A)\right)$ are isomorphic and are either empty or zero-dimensional by Theorem 3.14 and Corollary 3.19. It follows that the rings $\boldsymbol{k}[\boldsymbol{T}] / I_{2}(C)$ and $\boldsymbol{k}[\boldsymbol{u}] / I_{3}(A)$ have the same dimension and this common dimension is either 0 or 1 ; furthermore, these two rings have the same multiplicity. The height of $I_{3}(A)$ is 2 if and only if the gcd of $I_{3}(A)$ is a unit. Otherwise, $I_{3}(A)$ has height 1 and $\left(I_{3}(A)\right)^{\mathrm{unm}}=\left(\operatorname{gcd} I_{3}(A)\right)$. In this case,

$$
e\left(\boldsymbol{k}[\boldsymbol{u}] / I_{3}(A)\right)=e\left(\boldsymbol{k}[\boldsymbol{u}] /\left(\operatorname{gcd} I_{3}(A)\right)\right)=\operatorname{deg} \operatorname{gcd} I_{3}(A) .
$$

The equality $e\left(\boldsymbol{k}[\boldsymbol{T}] / I_{2}(C)\right)=6-\mu\left(I_{2}(C)\right)$, when ht $I_{2}(C)=2$, is proven in Lemma 3.20, because the hypothesis that some generalized column ideal of $C$ is zero-dimensional is established in part (3) of Corollary 3.19. This completes the proof of (1).

Assertion (2) follows from Theorem 3.14: if $\ell$ is a linear factor of $\operatorname{gcd} I_{3}(A)$, then $V(\ell)$ is a point in $\operatorname{Proj}\left(\boldsymbol{k}[\boldsymbol{u}] / I_{3}(A)\right)$ and the corresponding singular point on $\mathcal{C}$ of multiplicity $c$ is $\pi_{1} \circ \pi_{2}^{-1}(V(\ell))$ as described in Remark 3.17. 
Assertion (4) is an immediate consequence of (2) and (3). We now prove (3). Consider the factor $\ell_{1}$ of $\operatorname{gcd} I_{3}(A)$. Let $p$ be the point on $\mathcal{C}$ which corresponds to $\ell_{1}$ as described in the proof of (2). Now apply a linear automorphism of $\mathbb{P}^{2}$ to move the point $p$ to $(0,0,1)$. According to the General Lemma, the entries of the bottom row of the new $\varphi$ are linearly dependent. Now apply a column operation to make the bottom row become [0,*]. Under the correspondence of Remark 3.17 the point $(0,0,1)$ is paired with $(1,0)$ and henceforth $\ell_{1}$ has moved to $u_{2}$. Furthermore, $\varphi$ has become

$$
\varphi=\left[\begin{array}{cc}
Q_{1} & Q_{3} \\
Q_{2} & Q_{4} \\
0 & Q_{5}
\end{array}\right]
$$

where the $Q_{i}$ are forms of degree $c$. The fact that ht $(I)=2$ forces $Q_{1}$ and $Q_{2}$ to be linearly independent over $\boldsymbol{k}$. Write $Q_{i}=\rho^{(c)} \gamma_{i}$, where $\gamma_{i}$ is a column vector with entries in $\boldsymbol{k}$. A straightforward calculation yields

$$
A=\left[\begin{array}{lll}
u_{1} \gamma_{1}+u_{2} \gamma_{3} & u_{1} \gamma_{2}+u_{2} \gamma_{4} & u_{2} \gamma_{5}
\end{array}\right]
$$

It follows that

$$
I_{3}(A)=u_{2} I_{3}\left(A^{\prime}\right)
$$

for

$$
A^{\prime}=\left[\begin{array}{lll}
u_{1} \gamma_{1}+u_{2} \gamma_{3} & u_{1} \gamma_{2}+u_{2} \gamma_{4} & \gamma_{5}
\end{array}\right]
$$

Notice that

$$
I_{3}\left(A^{\prime}\right) \cong u_{1}^{2} I_{3}\left(\left[\begin{array}{lll}
\gamma_{1} & \gamma_{2} & \gamma_{5}
\end{array}\right]\right) \quad \bmod u_{2}
$$

hence, $u_{2}$ divides every element of $I_{3}\left(A^{\prime}\right)$ if and only if $\gamma_{1}, \gamma_{2}, \gamma_{5}$ are linearly dependent.

The fact that $\gamma_{1}$ and $\gamma_{2}$ are linearly independent guarantees that

$\gamma_{1}, \gamma_{2}, \gamma_{5}$ are linearly dependent

$\Longleftrightarrow \gamma_{5}$ is in the vector space spanned by $\gamma_{1}$ and $\gamma_{2}$

$\Longleftrightarrow Q_{5}$ is in the vector space spanned by $Q_{1}$ and $Q_{2}$.

By the Triple Lemma 2.1 this is equivalent to the existence of at least one singular point of multiplicity $c$ infinitely near to $(0,0,1)$. Thus such a point exists if and only if $e_{1} \geq 2$.

Assume now that there does exist a singular point of multiplicity $c$ infinitely near to $(0,0,1)$; in particular $Q_{5}$ is in the span of $Q_{1}$ and $Q_{2}$ and therefore applying row operations on $\varphi$ only involving the first two rows we may assume that $\gamma_{5}=\gamma_{2}$. Notice that $I_{3}(A)$ is unchanged. We have $I_{3}(A)=u_{2}^{2} I_{3}\left(A^{\prime \prime}\right)$ for 
$A^{\prime \prime}=\left[\begin{array}{lll}u_{1} \gamma_{1}+u_{2} \gamma_{3} & \gamma_{4} & \gamma_{2}\end{array}\right]$. Now one sees as before that $u_{2}$ divides every element of $I_{3}\left(A^{\prime \prime}\right)$ if and only if $\gamma_{1}, \gamma_{4}, \gamma_{2}$ are linearly dependent. Therefore, $u_{2}^{3}$ divides every element of $I_{3}(A)$ if and only if $\gamma_{1}, \gamma_{4}, \gamma_{2}$ are linearly dependent. The fact that $\gamma_{1}$ and $\gamma_{2}$ are linearly independent guarantees that

$\gamma_{1}, \gamma_{2}, \gamma_{4}$ are linearly dependent

$\Longleftrightarrow \gamma_{4}$ is in the vector space spanned by $\gamma_{1}$ and $\gamma_{2}$

$\Longleftrightarrow Q_{4}$ is in the vector space spanned by $Q_{1}$ and $Q_{2}$.

By the Triple Lemma 2.1 this is equivalent to the existence of exactly two singular points of multiplicity $c$ infinitely near to $(0,0,1)$. Thus such points exist if and only if $e_{1}=3$.

\section{Section 4. Singularities of Multiplicity \\ EQUAL TO DEGREE DIVIDED BY TWO.}

Throughout this section $B$ is the polynomial ring $\boldsymbol{k}[x, y]$, where $\boldsymbol{k}$ is a field, and $d=2 c$ is an even integer.

When $\boldsymbol{k}$ is algebraically closed, Theorem 4.8 completely classifies the parameterizations of rational plane curves $\mathcal{C}$ of degree $d$ as a function of the configuration of multiplicity $c$ singularities which appear on, or infinitely near, $\mathcal{C}$. Theorem 4.8 is typical of classification theorems in general in the sense that a classification theorem is always the culmination of one project and is often the starting point of new projects. Indeed, Theorem 4.8 is the starting point of the decomposition of $\mathbb{T}_{d}$ into strata which is carried out in Section 6. Also, we anticipate that Theorem 4.8 will eventually lead to a better understanding of the other singularities on and infinitely near the curve $\mathcal{C}$. An analysis of this sort for quartics is carried out in Section 8 . A future paper will contain our analysis of all singularities on sextics.

Let $\mathcal{C}$ be a rational plane curve of degree $d=2 c$. Recall, from Corollary 2.5, that if there is a multiplicity $c$ singularity on, or infinitely near, $\mathcal{C}$, then every entry in a homogeneous Hilbert-Burch matrix for a parameterization of $\mathcal{C}$ is a form of degree c. In Theorem 4.9 we decompose the space of all such Hilbert-Burch matrices (called $\mathrm{BalH}_{d}$, see Definition 4.3) into 11 disjoint orbits under the action of the group $G=\mathrm{GL}_{3}(\boldsymbol{k}) \times \mathrm{GL}_{2}(\boldsymbol{k})$. The orbits are parameterized by the poset ECP of Definition 4.6. If $\natural$ is in ECP, then the corresponding orbit is called $\mathrm{DO}_{\natural}^{\mathrm{Bal}}$. Each orbit $\mathrm{DO}_{\natural}^{\mathrm{Bal}}$ has the form $G \cdot M_{\natural}^{\mathrm{Bal}}$, where $M_{\natural}^{\mathrm{Bal}}$ is the intersection of $\mathrm{BalH}_{d}$ and an open subset of some affine space. Let $\varphi$ be a Hilbert-Burch matrix in $M_{\natural}^{\text {Bal }}$, for some $\downarrow$, and let $\mathcal{C}$ be the corresponding curve. In Lemma 4.10 we apply Theorem 3.22 and Corollary 2.5 to explicitly identify the multiplicity $c$ singularities on $\mathcal{C}$, together with all infinitely near multiplicity $c$ singularities. 
We notice that Theorem 3.22 guarantees that the number of distinct singular points of multiplicity $c$ that are either on $\mathcal{C}$ or infinitely near to $\mathcal{C}$ is at most 3 since this number is deg gcd $I_{3}(A)$ and $I_{3}(A)$ is generated by cubic forms. Of course, this bound is also implied by Max Noether's formula

$$
g=\left(\begin{array}{c}
d-1 \\
2
\end{array}\right)-\sum_{q}\left(\begin{array}{c}
m_{q} \\
2
\end{array}\right)
$$

which gives the genus $g$ of the irreducible plane curve $\mathcal{C}$ of degree $d$, where $q$ varies over all singularities and infinitely near singularities of $\mathcal{C}$, and $m_{q}$ is the multiplicity at $q$. (See, for example, chapter 4 exercise 1.8 and chapter 5 examples 3.9 .2 and 3.9.3 in [16].) At any rate, there are seven possible configurations of multiplicity $c$ singularities. The curve itself might have $0,1,2$, or 3 singularities of multiplicity $c$ and any one of these singularities has 0,1 , or 2 infinitely near singularities of multiplicity $c$, provided that the total number is at most 3 . The seven possibilities are: $\emptyset,\{c\},\{c, c\},\{c, c, c\},\{c: c\},\{c: c, c\}$, and $\{c: c: c\}$, where a colon indicates an infinitely near singularity, a comma indicates a different singularity on the curve, and $\emptyset$ indicates that there are no singularities of multiplicity $c$ on, or infinitely near, $\mathcal{C}$. Henceforth, we refer to the set of seven possible configurations of multiplicity $c$ singularities as CP. The order that we impose on this set is dictated by the decomposition of $\mathbb{T}_{d}$ into strata which takes place in Section 6 .

Definition 4.2. Let $(\mathrm{CP}, \leq)$ be the Configuration Poset. The elements of $\mathrm{CP}$ are the seven possible configurations for multiplicity $c$ singularities on or infinitely near a rational plane curve of degree $d=2 c$. We read "\#" as the sharp symbol and we write $\#^{\prime} \rightarrow \#$, for $\#^{\prime}$ and $\#$ in $\mathrm{CP}$, to mean $\#^{\prime} \leq \#$. The poset $\mathrm{CP}$ is:

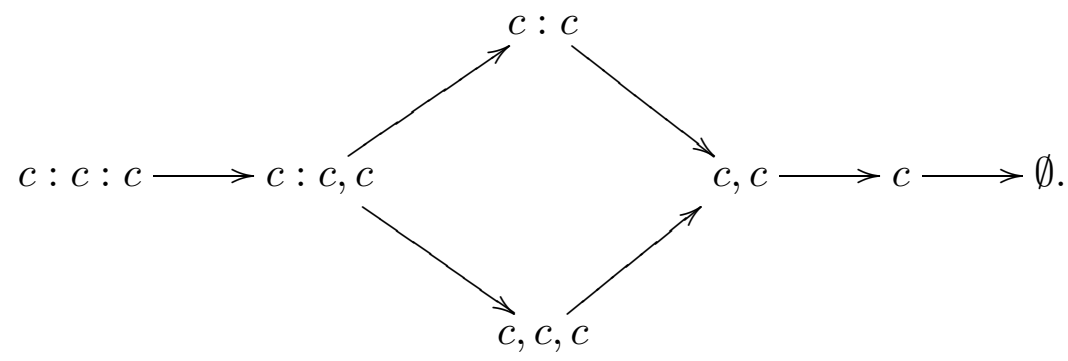

Definition 4.3. Let $B$ be the polynomial ring $\boldsymbol{k}[x, y]$, over the field $\boldsymbol{k}$, and $d$ be the even integer $2 c$.

(1) Define $\mathbb{H}_{d}$ to be the space of $3 \times 2$ matrices with entries from $B_{c}$.

(2) Define $\mathrm{BalH}_{d}=\left\{\varphi \in \mathbb{H}_{d} \mid\right.$ ht $\left.I_{2}(\varphi)=2\right\}$. 
(3) Define

$$
\mathbb{B H}_{d}=\left\{\begin{array}{l|l}
\varphi \in \mathrm{BalH}_{d} & \begin{array}{l}
\text { the morphism } \Psi: \mathbb{P}^{1} \rightarrow \mathcal{C} \text { determined by the signed } \\
\text { maximal order minors of } \varphi \text { is birational }
\end{array}
\end{array}\right\} .
$$

(4) The group $G=\mathrm{GL}_{3}(\boldsymbol{k}) \times \mathrm{GL}_{2}(\boldsymbol{k})$ acts on $\mathbb{H}_{d}$. If $g=(\chi, \xi) \in G$ and $\varphi \in \mathbb{H}_{d}$, then $g \varphi=\chi \varphi \xi^{-1}$.

Remarks 4.4. (1) The variety $\mathbb{H}_{d}$ is isomorphic to affine space $\mathbb{A}^{6 c+6}$. The subsets $\mathbb{B H}_{d}$ and $\mathrm{BalH}_{d}$ of $\mathbb{H}_{d}$ are both open, see Observation 5.29.

(2) If

$$
\varphi=\left[\begin{array}{ll}
Q_{1,1} & Q_{1,2} \\
Q_{2,1} & Q_{2,2} \\
Q_{3,1} & Q_{3,2}
\end{array}\right]
$$

is a $3 \times 2$ matrix $\varphi$ with entries in $B$, then the ordered triple of "signed maximal order minors" of $\varphi$ is the following ordered triple $\Phi(\varphi)$ of polynomials from $B$ :

$$
\Phi(\varphi)=\left(\left|\begin{array}{ll}
Q_{2,1} & Q_{2,2} \\
Q_{3,1} & Q_{3,2}
\end{array}\right|,-\left|\begin{array}{ll}
Q_{1,1} & Q_{1,2} \\
Q_{3,1} & Q_{3,2}
\end{array}\right|,\left|\begin{array}{ll}
Q_{1,1} & Q_{1,2} \\
Q_{2,1} & Q_{2,2}
\end{array}\right|\right)
$$

(3) If $\varphi$ is in $\mathrm{BalH}_{d}$, then $\varphi$ is a Hilbert-Burch matrix for the row vector determined by the signed maximal order minors of $\varphi$; furthermore, $\varphi$ is balanced in the sense that every element of $\varphi$ is a homogeneous element of the same degree. We refer to $\mathrm{BalH}_{d}$ as the space of "Balanced Hilbert-Burch matrices for triples of homogeneous $d$-forms".

(4) If $\varphi$ is in $\mathbb{H}_{d}$, then

$$
\begin{aligned}
& \varphi \text { is a Balanced Hilbert-Burch matrix and the morphism } \\
& \varphi \in \mathbb{B H}_{d} \Longleftrightarrow \text { determined by the signed maximal order minors of } \varphi \text { is } \\
& \text { Birational and Base point free. }
\end{aligned}
$$

We refer to $\mathbb{B H}_{d}$ as the space of "Balanced Hilbert-Burch matrices for true triples of homogeneous $d$-forms". The notion of "true triples" is introduced in Remark 5.5. In practice we are interested in the geometry which corresponds to $\mathbb{B H}_{d}$. On the other hand, Theorem 4.9 and two thirds of Lemma 4.10 do not require the birationality hypothesis. These results make sense in $\mathrm{BalH}_{d}$.

(5) The action of $G$ on $\mathbb{H}_{d}$ restricts to given actions of $G$ on $\mathrm{BalH}_{d}$ and also on $\mathbb{B H}_{d}$. Indeed, $I_{2}(\varphi)$ and $I_{2}(g \varphi)$ are equal for all $g \in G$ and $\varphi \in \mathbb{H}_{d}$ and the curve parameterized by the signed maximal order minors of $g \varphi$ is the image, under a 
linear automorphism of $\mathbb{P}^{2}$ of the curve parameterized by the signed maximal order minors of $\varphi$; see Remark 0.9 .

(6) The well known formula $\xi^{-1}=(\operatorname{det} \xi)^{-1} \operatorname{Adj} \xi$, where $\operatorname{Adj} \xi$ is the classical adjoint of $\xi$, expresses the inverse of the matrix as a rational function in the entries of $\xi$. Thus, the function $\Upsilon: G \times \mathbb{H}_{d} \rightarrow \mathbb{H}_{d}$, which is defined by $\Upsilon(g, \varphi)=g \varphi$, is a morphism of varieties.

There are 7 configurations of multiplicity $c$ singularities in $\mathrm{CP}$, but 11 disjoint orbits in our decomposition of $\mathrm{BalH}_{d}$. We form the Total Configuration Poset (TCP) by adjoining 6 new elements to $\mathrm{CP}$ and the Extended Configuration Poset (ECP) by removing 2 elements of CP from TCP. The order in TCP is used when we combine some of the disjoint orbits $\left\{\mathrm{DO}_{\natural} \mid \downarrow \in \mathrm{ECP}\right\}$ to form $\left\{\mathrm{CO}_{\#} \mid \# \in \mathrm{CP}\right\}$ in Definition 4.17.

Definition 4.6. As a set, TCP consists of CP together with 6 new elements:

$$
\mu_{2}, \quad\left(c, \mu_{4}\right), \quad\left(c, \mu_{5}\right), \quad\left(\emptyset, \mu_{4}\right), \quad\left(\emptyset, \mu_{5}\right), \quad \text { and } \quad\left(\emptyset, \mu_{6}\right) .
$$

The order in $\mathrm{CP}$ is extended to give the order in TCP:

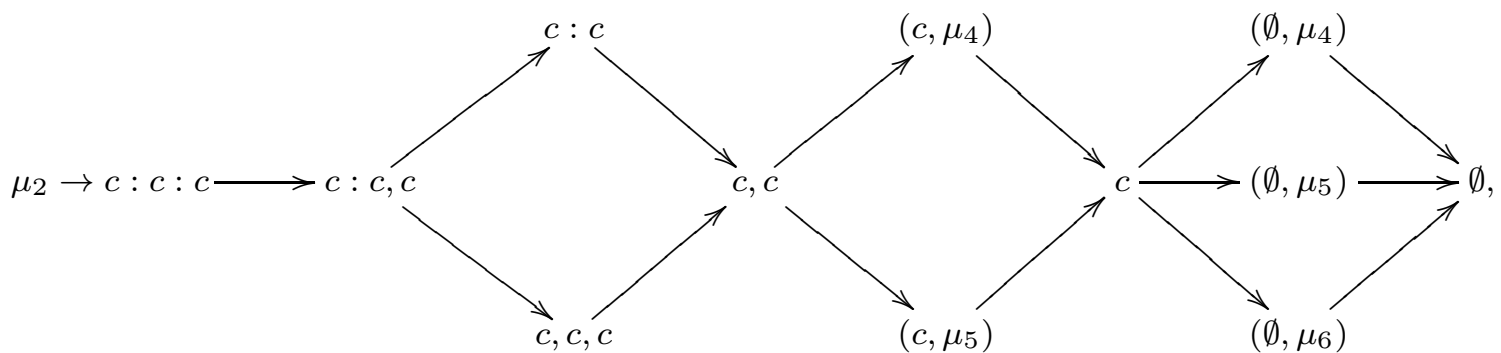

where we write $\natural^{\prime} \rightarrow \natural$, for $\natural^{\prime}$ and $\downarrow$ in TCP to mean $\natural^{\prime} \leq \downarrow$. The symbol " $\natural^{\prime}$ " is read as "natural". The poset ECP is TCP with $c$ and $\emptyset$ removed. So ECP is

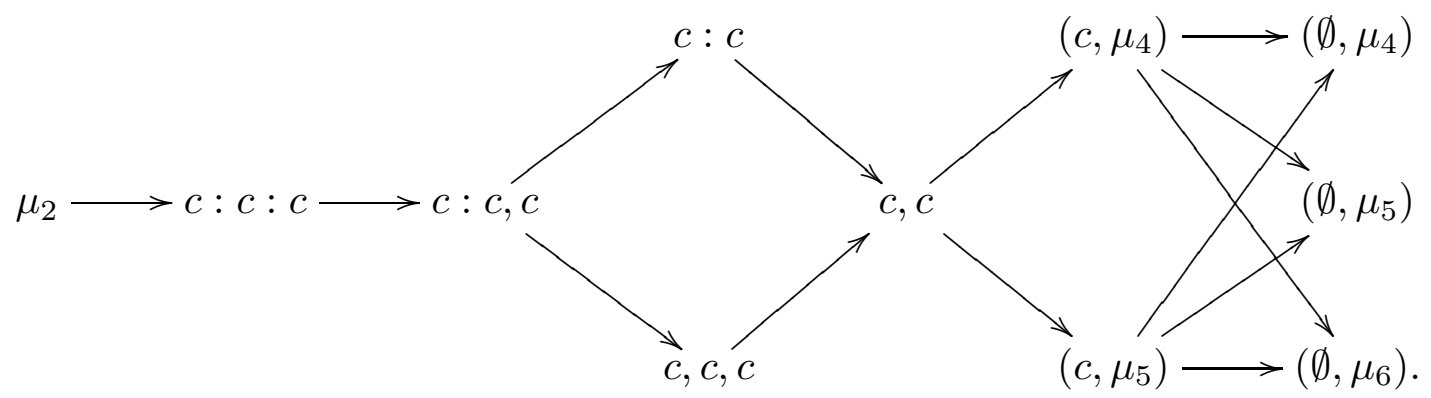


Definition 4.7. (1) For each $\downarrow \in \mathrm{ECP}$, let $M_{\natural}^{\mathrm{Bal}}$ be the following subset of $\mathrm{BalH}_{d}$ :

$$
\begin{aligned}
& M_{\mu_{2}}^{\mathrm{Bal}}=\left\{\left[\begin{array}{cc}
Q_{1} & 0 \\
Q_{2} & Q_{1} \\
0 & Q_{2}
\end{array}\right] \in \mathrm{BalH}_{d} \mid \operatorname{dim}_{\boldsymbol{k}}<Q_{1}, Q_{2}>=2\right\}, \\
& M_{c: c: c}^{\mathrm{Bal}}=\left\{\left[\begin{array}{cc}
Q_{1} & Q_{2} \\
Q_{3} & Q_{1} \\
0 & Q_{3}
\end{array}\right] \in \mathrm{BalH}_{d} \mid \operatorname{dim}_{\boldsymbol{k}}<Q_{1}, Q_{2}, Q_{3}>=3\right\} \text {, } \\
& M_{c: c, c}^{\mathrm{Bal}}=\left\{\left[\begin{array}{cc}
Q_{1} & 0 \\
Q_{2} & Q_{3} \\
0 & Q_{2}
\end{array}\right] \in \mathrm{BalH}_{d} \mid \operatorname{dim}_{\boldsymbol{k}}<Q_{1}, Q_{2}, Q_{3}>=3\right\} \text {, } \\
& M_{c, c, c}^{\mathrm{Bal}}=\left\{\left[\begin{array}{cc}
Q_{1} & Q_{1} \\
Q_{2} & 0 \\
0 & Q_{3}
\end{array}\right] \in \mathrm{BalH}_{d} \mid \operatorname{dim}_{\boldsymbol{k}}<Q_{1}, Q_{2}, Q_{3}>=3\right\} \text {, } \\
& M_{c: c}^{\mathrm{Bal}}=\left\{\left[\begin{array}{cc}
Q_{1} & Q_{2} \\
Q_{3} & Q_{4} \\
0 & Q_{3}
\end{array}\right] \in \mathrm{BalH}_{d} \mid \operatorname{dim}_{\boldsymbol{k}}<Q_{1}, Q_{2}, Q_{3}, Q_{4}>=4\right\}, \\
& M_{c, c}^{\mathrm{Bal}}=\left\{\left[\begin{array}{cc}
Q_{1} & Q_{2} \\
Q_{3} & Q_{3} \\
0 & Q_{4}
\end{array}\right] \in \mathrm{BalH}_{d} \mid \operatorname{dim}_{\boldsymbol{k}}<Q_{1}, Q_{2}, Q_{3}, Q_{4}>=4\right\}, \\
& M_{\left(c, \mu_{4}\right)}^{\mathrm{Bal}}=\left\{\left[\begin{array}{cc}
Q_{1} & Q_{2} \\
Q_{3} & Q_{1} \\
0 & Q_{4}
\end{array}\right] \in \mathrm{BalH}_{d} \mid \operatorname{dim}_{\boldsymbol{k}}<Q_{1}, Q_{2}, Q_{3}, Q_{4}>=4\right\}, \\
& M_{\left(c, \mu_{5}\right)}^{\mathrm{Bal}}=\left\{\left[\begin{array}{cc}
Q_{1} & Q_{2} \\
Q_{3} & Q_{4} \\
0 & Q_{5}
\end{array}\right] \in \mathrm{BalH}_{d} \mid \operatorname{dim}_{\boldsymbol{k}}<Q_{1}, Q_{2}, Q_{3}, Q_{4}, Q_{5}>=5\right\} \text {, } \\
& M_{\left(\emptyset, \mu_{4}\right)}^{\mathrm{Bal}}=\left\{\left[\begin{array}{ll}
Q_{1} & Q_{2} \\
Q_{2} & Q_{3} \\
Q_{3} & Q_{4}
\end{array}\right] \in \mathrm{BalH}_{d} \mid \operatorname{dim}_{\boldsymbol{k}}<Q_{1}, Q_{2}, Q_{3}, Q_{4}>=4\right\} \text {, } \\
& M_{\left(\emptyset, \mu_{5}\right)}^{\mathrm{Bal}}=\left\{\left[\begin{array}{ll}
Q_{1} & Q_{2} \\
Q_{3} & Q_{4} \\
Q_{5} & Q_{1}
\end{array}\right] \in \mathrm{BalH}_{d} \mid \operatorname{dim}_{\boldsymbol{k}}<Q_{1}, Q_{2}, Q_{3}, Q_{4}, Q_{5}>=5\right\} \text {, and } \\
& M_{\left(\emptyset, \mu_{6}\right)}^{\mathrm{Bal}}=\left\{\left[\begin{array}{ll}
Q_{1} & Q_{2} \\
Q_{3} & Q_{4} \\
Q_{5} & Q_{6}
\end{array}\right] \in \mathrm{BalH}_{d} \mid \operatorname{dim}_{\boldsymbol{k}}<Q_{1}, Q_{2}, Q_{3}, Q_{4}, Q_{5}, Q_{6}>=6\right\} \text {. }
\end{aligned}
$$

(2) For each $\natural \in \mathrm{ECP}$, let $\mathrm{DO}_{\natural}^{\mathrm{Bal}}$ be the subset $G \cdot M_{\natural}^{\mathrm{Bal}}=\left\{g \varphi \mid g \in G, \varphi \in M_{\natural}^{\mathrm{Bal}}\right\}$ 
of $\mathrm{BalH}_{d}$.

(3) For each $\natural \in \mathrm{ECP}$, let $M_{\natural}=M_{\natural}^{\mathrm{Bal}} \cap \mathbb{B H}_{d}$ and $\mathrm{DO}_{\natural}=\mathrm{DO}_{\natural}^{\mathrm{Bal}} \cap \mathbb{B H}_{d}$.

(4) Define $M_{c}=M_{\left(c, \mu_{4}\right)} \cup M_{\left(c, \mu_{5}\right)}$.

Remarks. (1) Notice that each set $M_{\natural}^{\mathrm{Bal}}$ and $M_{\natural}$ has the form $\mathrm{BalH}_{d} \cap U$ or $\mathbb{B H}_{d} \cap U$, where $U$ is an open subset of some affine space. Indeed, for example the condition " $\operatorname{dim}_{k}<Q_{1}, Q_{2}, Q_{3}>=3$ " is equivalent to the statement that the coefficients of $Q_{1}, Q_{2}, Q_{3}$ are not solutions of the of the maximal order minors of a generic $3 \times(c+1)$ matrix.

(2) We think of (4) from the above definition as an abbreviation. We don't use this abbreviation until (6.5) where its use does simplify the exposition.

Theorem 4.8. Let $\mathcal{C}$ be a rational plane curve of even degree $d=2 c$ over an algebraically closed field $\boldsymbol{k}$. Assume that there exists at least one singularity of multiplicity $c$ on or infinitely near $\mathcal{C}$. Then there exists a linear automorphism $\Lambda$ of $\mathbb{P}^{2}$ and a matrix $\varphi$ in $M_{\natural}$, for some $\downarrow \in \operatorname{ECP} \backslash\left\{\mu_{2},\left(\emptyset, \mu_{4}\right),\left(\emptyset, \mu_{5}\right),\left(\emptyset, \mu_{6}\right)\right\}$, such that $\Lambda \mathcal{C}$ is parameterized by the signed maximal order minors $\varphi$. Furthermore, the following statements hold.

(1) If the configuration of multiplicity $c$ singularities on or infinitely near $\mathcal{C}$ is described by $\{c\}$, then $\Lambda \mathcal{C}$ is parameterized by the signed maximal order minors of $\varphi$ in $M_{\left(c, \mu_{4}\right)}$ or $M_{\left(c, \mu_{5}\right)}$. In this case, $[0: 0: 1]$ is the singularity of multiplicity $c$.

(2) If the configuration of multiplicity $c$ singularities on or infinitely near $\mathcal{C}$ is described by $\{c, c\}$, then $\Lambda \mathcal{C}$ is parameterized by the signed maximal order minors of $\varphi \in M_{c, c}$. In this case, $p_{1}=[0: 0: 1]$ and $p_{2}=[0: 1: 0]$ are the singularities of multiplicity $c$.

(3) If the configuration of multiplicity $c$ singularities on or infinitely near $\mathcal{C}$ is described by $\{c, c, c\}$, then $\Lambda \mathcal{C}$ is parameterized by the signed maximal order minors of $\varphi \in M_{c, c, c}$. In this case, $p_{1}=[0: 0: 1], p_{2}=[0: 1: 0]$, and $p_{3}=[1: 0: 0]$, are the singularities on $\mathcal{C}$ of multiplicity $c$.

(4) If the configuration of multiplicity $c$ singularities on or infinitely near $\mathcal{C}$ is described by $\{c: c\}$, then $\Lambda \mathcal{C}$ is parameterized by the signed maximal order minors of $\varphi \in M_{c: c}$. In this case, $p=[0: 0: 1]$ is the singularity on $\mathcal{C}$ of multiplicity $c$ and there is one singularity of multiplicity $c$ infinitely near to $p$.

(5) If the configuration of multiplicity $c$ singularities on or infinitely near $\mathcal{C}$ is described by $\{c: c, c\}$, then $\Lambda \mathcal{C}$ is parameterized by the signed maximal order minors of $\varphi \in M_{c: c, c}$. In this case, $p_{1}=[0: 0: 1]$ and $p_{2}=[1: 0: 0]$ are the singularities on $\mathcal{C}$ of multiplicity $c$ and there is one singularity of multiplicity $c$ infinitely near to $p_{1}$. 
(6) If the configuration of multiplicity $c$ singularities on or infinitely near $\mathcal{C}$ is described by $\{c: c: c\}$, then $\Lambda \mathcal{C}$ is parameterized by the signed maximal order minors of $\varphi \in M_{c: c: c .}$. In this case, $p_{1}=[0: 0: 1]$ is the singularity on $\mathcal{C}$ of multiplicity $c$ and there are two singularities of multiplicity $c$ infinitely near to $p_{1}$.

Proof. Recall from Corollary 2.5, that if there is a singularity of multiplicity $c$ on or infinitely near $\mathcal{C}$, then there is a singularity of multiplicity $c$ on $\mathcal{C}$ and every entry in a homogeneous Hilbert-Burch matrix for $\mathcal{C}$ is a form of degree $c$. Thus, $\mathcal{C}$ is birationally parameterized by the signed maximal order minors of some matrix $\varphi$ in $\mathbb{B H}_{d}$. Theorem 4.9 shows that there exists $g \in G$ and $\varphi_{\natural} \in M_{\natural}$ for some $\downarrow \in$ ECP with $\varphi=g \varphi_{\natural}$. Thus, there is a linear automorphism of $\mathbb{P}^{2}$ which carries $\mathcal{C}$ to the curve $\mathcal{C}_{\natural}$ which is parameterized by the signed maximal order minors of $\varphi_{\natural}$, see Remark 0.9. Part (3) of Lemma 4.10 shows that $\downarrow \notin\left\{\mu_{2},\left(\emptyset, \mu_{6}\right),\left(\emptyset, \mu_{5}\right),\left(\emptyset, \mu_{4}\right)\right\}$. Part (3) of Lemma 4.10 also records the multiplicity $c$ singularities on or infinitely near $\mathcal{C}_{\natural}$.

Theorem 4.9. If every polynomial in $\boldsymbol{k}[x]$ of degree 2 or 3 has a root in the field $k$, then the space of balanced Hilbert-Burch matrices $\mathrm{BalH}_{d}$ is the disjoint union of the orbits $\mathrm{DO}_{\natural}^{\mathrm{Bal}}$ as $\emptyset$ varies over $\mathrm{ECP}$.

Proof. Fix $\varphi \in \mathrm{BalH}_{d}$. We first prove that there exists $g \in G$ with $g \varphi \in M_{\natural}^{\text {Bal }}$ for some $\downarrow \in \mathrm{ECP}$. Consider the parameter $\mu=\mu\left(I_{1}(\varphi)\right)$. The matrix $\varphi$ has six homogeneous entries of degree $c$, so $\mu \leq 6$. On the other hand, the hypothesis that ht $I_{2}(\varphi)=2$ guarantees that $2 \leq \mu$. Thus, $2 \leq \mu \leq 6$. We treat each possible value for $\mu$ separately. If $\mu=6$, then the entries of $\varphi$ are linearly independent and $\varphi \in M_{\left(\emptyset, \mu_{6}\right)}^{\mathrm{Bal}}$. Suppose now that $\mu=5$. If $\varphi$ has a generalized zero (see Remark $0.7)$, then, after row and column operations, $\varphi$ is transformed into $g \varphi \in M_{\left(c, \mu_{5}\right)}^{\mathrm{Bal}}$. If $\varphi$ does not have a generalized zero, one may apply row and column operations to put $\varphi$ in the form

$$
\left[\begin{array}{cc}
Q_{1} & Q_{4} \\
Q_{2} & Q_{5} \\
Q_{3} & \sum_{i=1}^{5} \alpha_{i} Q_{i}
\end{array}\right]
$$

where the $\alpha_{i} \in k$ are constants. Further row and column operations (and re-naming the entries of $\varphi$ ) put $\varphi$ in the form

$$
\left[\begin{array}{cc}
Q_{1} & Q_{4} \\
Q_{2} & Q_{5} \\
Q_{3} & \sum_{i=1}^{2} \alpha_{i} Q_{i}
\end{array}\right]
$$


and ultimately one finds $g \in G$ with $g \varphi \in M_{\left(\emptyset, \mu_{5}\right)}^{\mathrm{Bal}}$. Lemmas 4.13 and 4.14 show that if $\mu$ is equal to 4 or 3 , then there exists $g \in G$ with $g \varphi \in M_{\natural}^{\text {Bal }}$ for some $\natural \in$ ECP. Finally, if $\mu=2$, then one quickly puts $\varphi$ in the form

$$
\left[\begin{array}{cc}
* & * \\
* & * \\
0 & Q_{2}
\end{array}\right]
$$

Further elementary row and column operations transform $\varphi$ into

$$
\left[\begin{array}{cc}
Q_{1} & \alpha Q_{1} \\
Q_{2} & \beta Q_{1} \\
0 & Q_{2}
\end{array}\right] \text {, then }\left[\begin{array}{cc}
Q_{1} & 0 \\
Q_{2} & \beta Q_{1} \\
0 & Q_{2}
\end{array}\right]
$$

for some constants $\alpha$ and $\beta$. The constant $\beta$ is non-zero since ht $I_{2}(\varphi)=2$ and there exists $g \in G$ with $g \varphi \in M_{\mu_{2}}^{\mathrm{Bal}}$. We have shown that the $\mathrm{BalH}_{d}=\cup_{\natural \in \mathrm{ECP}} \mathrm{DO}_{\natural}^{\mathrm{Bal}}$. The chart of invariants in (2) of Lemma 4.10 shows that the orbits $\mathrm{DO}_{\natural}^{\mathrm{Bal}}$, with $\natural \in \mathrm{ECP}$, are disjoint.

Lemma 4.10. Let $\varphi_{\natural}, C_{\natural}$, and $A_{\natural}$ be matrices which satisfy (3.11) and (3.12) with $\varphi_{\natural} \in \mathrm{DO}_{\natural}^{\mathrm{Bal}}$.

(1) One may transform the matrices $\left(C_{\natural}, A_{\natural}\right)$, using elementary operations and the suppression of zero rows, into the matrices $\left(C_{\natural}^{\prime}, A_{\natural}^{\prime}\right)$, which are given by:

$$
\begin{aligned}
& C_{\left(\emptyset, \mu_{6}\right)}^{\prime}=\left[\begin{array}{cc}
T_{1} & 0 \\
T_{2} & 0 \\
T_{3} & 0 \\
0 & T_{1} \\
0 & T_{2} \\
0 & T_{3}
\end{array}\right], A_{\left(\emptyset, \mu_{6}\right)}^{\prime}=\left[\begin{array}{ccc}
u_{1} & 0 & 0 \\
0 & u_{1} & 0 \\
0 & 0 & u_{1} \\
u_{2} & 0 & 0 \\
0 & u_{2} & 0 \\
0 & 0 & u_{2}
\end{array}\right], C_{\left(\emptyset, \mu_{5}\right)}^{\prime}=\left[\begin{array}{cc}
T_{1} & T_{3} \\
T_{2} & 0 \\
T_{3} & 0 \\
0 & T_{1} \\
0 & T_{2}
\end{array}\right], A_{\left(\emptyset, \mu_{5}\right)}^{\prime}=\left[\begin{array}{ccc}
u_{1} & 0 & u_{2} \\
0 & u_{1} & 0 \\
0 & 0 & u_{1} \\
u_{2} & 0 & 0 \\
0 & u_{2} & 0
\end{array}\right], \\
& C_{\left(c, \mu_{5}\right)}^{\prime}=\left[\begin{array}{cc}
T_{1} & 0 \\
T_{2} & 0 \\
0 & T_{1} \\
0 & T_{2} \\
0 & T_{3}
\end{array}\right], A_{\left(c, \mu_{5}\right)}^{\prime}=\left[\begin{array}{ccc}
u_{1} & 0 & 0 \\
0 & u_{1} & 0 \\
u_{2} & 0 & 0 \\
0 & u_{2} & 0 \\
0 & 0 & u_{2}
\end{array}\right], C_{\left(\emptyset, \mu_{4}\right)}^{\prime}=\left[\begin{array}{cc}
T_{1} & 0 \\
T_{2} & T_{1} \\
T_{3} & T_{2} \\
0 & T_{3}
\end{array}\right], A_{\left(\emptyset, \mu_{4}\right)}^{\prime}=\left[\begin{array}{ccc}
u_{1} & 0 & 0 \\
u_{2} & u_{1} & 0 \\
0 & u_{2} & u_{1} \\
0 & 0 & u_{2}
\end{array}\right], \\
& C_{\left(c, \mu_{4}\right)}^{\prime}=\left[\begin{array}{cc}
T_{1} & T_{2} \\
T_{2} & 0 \\
0 & T_{1} \\
0 & T_{3}
\end{array}\right], A_{\left(c, \mu_{4}\right)}^{\prime}=\left[\begin{array}{ccc}
u_{1} & u_{2} & 0 \\
0 & u_{1} & 0 \\
u_{2} & 0 & 0 \\
0 & 0 & u_{2}
\end{array}\right], C_{c, c}^{\prime}=\left[\begin{array}{cc}
T_{1} & 0 \\
0 & T_{1} \\
T_{2} & T_{2} \\
0 & T_{3}
\end{array}\right], A_{c, c}^{\prime}=\left[\begin{array}{ccc}
u_{1} & 0 & 0 \\
u_{2} & 0 & 0 \\
0 & \left(u_{1}+u_{2}\right) & 0 \\
0 & 0 & u_{2}
\end{array}\right], \\
& C_{c: c}^{\prime}=\left[\begin{array}{cc}
T_{1} & 0 \\
T_{2} & T_{3} \\
0 & T_{1} \\
0 & T_{2}
\end{array}\right], A_{c: c}^{\prime}=\left[\begin{array}{ccc}
u_{1} & 0 & 0 \\
0 & u_{1} & u_{2} \\
u_{2} & 0 & 0 \\
0 & u_{2} & 0
\end{array}\right], C_{c: c: c}^{\prime}=\left[\begin{array}{cc}
T_{1} & T_{2} \\
T_{2} & T_{3} \\
0 & T_{1}
\end{array}\right], A_{c: c: c}^{\prime}=\left[\begin{array}{ccc}
u_{1} & u_{2} & 0 \\
0 & u_{1} & u_{2} \\
u_{2} & 0 & 0
\end{array}\right], \\
& C_{c: c, c}^{\prime}=\left[\begin{array}{cc}
T_{1} & 0 \\
T_{2} & T_{3} \\
0 & T_{2}
\end{array}\right], A_{c: c, c}^{\prime}=\left[\begin{array}{ccc}
u_{1} & 0 & 0 \\
0 & u_{1} & u_{2} \\
0 & u_{2} & 0
\end{array}\right], C_{c, c, c}^{\prime}=\left[\begin{array}{cc}
T_{1} & T_{1} \\
T_{2} & 0 \\
0 & T_{3}
\end{array}\right], A_{c, c, c}^{\prime}=\left[\begin{array}{ccc}
u_{1}+u_{2} & 0 & 0 \\
0 & u_{1} & 0 \\
0 & 0 & u_{2}
\end{array}\right], \\
& C_{\mu_{2}}^{\prime}=\left[\begin{array}{ll}
T_{1} & T_{2} \\
T_{2} & T_{3}
\end{array}\right], A_{\mu_{2}}^{\prime}=\left[\begin{array}{ccc}
u_{1} & u_{2} & 0 \\
0 & u_{1} & u_{2}
\end{array}\right] \text {. }
\end{aligned}
$$


(2) The matrices $\left(\varphi_{\natural}, C_{\natural}, A_{\natural}\right)$ satisfy

$\begin{array}{cccc}\natural & \mu\left(I_{1}\left(\varphi_{\natural}\right)\right) & \mu\left(I_{2}\left(C_{\natural}\right)\right) & \operatorname{gcd}\left(I_{3}\left(A_{\natural}\right)\right) \\ \left(\emptyset, \mu_{6}\right) & 6 & 6 & 1 \\ \left(\emptyset, \mu_{5}\right) & 5 & 6 & 1 \\ \left(c, \mu_{5}\right) & 5 & 5 & \ell_{1} \\ \left(\emptyset, \mu_{4}\right) & 4 & 6 & 1 \\ \left(c, \mu_{4}\right) & 4 & 5 & \ell_{1} \\ c, c & 4 & 4 & \ell_{1} \ell_{2} \\ c: c & 4 & 4 & \ell_{1}^{2} \\ c, c, c & 3 & 3 & \ell_{1} \ell_{2} \ell_{3} \\ c: c, c & 3 & 3 & \ell_{1}^{2} \ell_{2} \\ c: c: c & 3 & 3 & \ell_{1}^{3} \\ \mu_{2} & 2 & 1 & 0,\end{array}$

for some non-associate linear forms $\ell_{1}, \ell_{2}, \ell_{3}$ in $\boldsymbol{k}[\boldsymbol{u}]$.

(3) If $\varphi_{\natural}$ is in $M_{\natural}$ and $\mathcal{C}_{\natural}$ is the curve parameterized by the signed maximal ordered minors of $\varphi_{\natural}$, then the multiplicity c singularities on or infinitely near $\mathcal{C}_{\natural}$ are given in the following chart.

\begin{tabular}{|c|c|c|}
\hline$\square$ & $\begin{array}{l}\text { the multiplicity } c \\
\text { singularities } p \text { on } \mathcal{C}_{i}\end{array}$ & $\begin{array}{l}\text { the number of multiplicity } c \\
\text { singularities infinitely near to } p\end{array}$ \\
\hline$\left(\emptyset, \mu_{6}\right)$ & none & \\
\hline$\left(\emptyset, \mu_{5}\right)$ & none & \\
\hline$\left(c, \mu_{5}\right)$ & {$[0: 0: 1]$} & 0 \\
\hline$\left(\emptyset, \mu_{4}\right)$ & none & \\
\hline$\left(c, \mu_{4}\right)$ & {$[0: 0: 1]$} & 0 \\
\hline \multicolumn{3}{|l|}{$c, c$} \\
\hline & {$[0: 0: 1]$} & 0 \\
\hline & {$[0: 1: 0]$} & 0 \\
\hline$c: c$ & {$[0: 0: 1]$} & 1 \\
\hline$c: c: c$ & {$[0: 0: 0: 1]$} & 2 \\
\hline \multicolumn{3}{|l|}{$c: c, c$} \\
\hline & {$[0: 0: 1]$} & 1 \\
\hline & {$[1: 0: 0]$} & 0 \\
\hline \multicolumn{3}{|l|}{$c, c, c$} \\
\hline & {$[0: 0: 1]$} & 0 \\
\hline & {$[0: 1: 0]$} & 0 \\
\hline & {$[1: 0: 0]$} & 0 \\
\hline
\end{tabular}




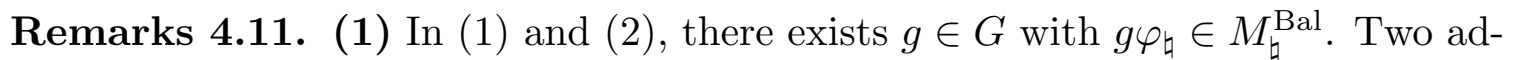
ditional hypotheses have been imposed on the matrix $\varphi_{\natural}$ in part (3). First of all, $\varphi_{\natural}$ is already in $M_{\natural}^{\text {Bal }}$; that is, $g$ may be taken to be 1 . Secondly, the parameterization determined by $\varphi_{\natural}$ is birational; that is, $\varphi_{\natural} \in \mathbb{B H}_{d}$; thus, $\varphi_{\natural} \in M_{\natural}^{\text {Bal }} \cap \mathbb{B H}_{d}=M_{\natural}$.

(2) To read the chart of (3), notice that the chart says for example, that there are 2 multiplicity $c$ singularities on the curve $\mathcal{C}_{c: c, c}$; one of these singularities $([0: 0: 1])$ has an infinitely near singularity of multiplicity $c$ and the other singularity $([1: 0: 0])$ does not have any infinitely near singularities of multiplicity $c$.

(3) We did not include $\mu_{2}$ in the chart of (3) because the intersection $\mathrm{DO}_{\mu_{2}}^{\mathrm{Bal}} \cap \mathbb{B H}_{d}$, which is also called $\mathrm{DO}_{\mu_{2}}$, is empty.

(4) Fix $\downarrow$ in ECP with $\downarrow \neq \mu_{2}$. We note that $\mathrm{DO}_{\natural}$ is a non-empty open subset of $\mathrm{DO}_{\natural}^{\mathrm{Bal}}$; see Observation 5.29 and Proposition 4.21. We also note that, according to Observation 0.11, if $c$ is a prime integer, then $\mathrm{DO}_{\natural}=\mathrm{DO}_{\natural}^{\mathrm{Bal}}$.

Proof. By the definition of $\mathrm{DO}_{d}^{\mathrm{Bal}}$, there is an element $g \in G$ with $g \varphi_{\natural} \in M_{\natural}^{\mathrm{Bal}}$. Remark 3.21 shows how the matrices $C_{g \natural}$ and $A_{g \natural}$ are obtained from $C_{\natural}$ and $A_{\natural}$. It suffices to prove the result when $\varphi_{\natural} \in M_{\natural}^{\mathrm{Bal}}$.

We have recorded the matrices $\left(C_{\natural}^{\prime}, A_{\natural}^{\prime}\right)$, whose entries are linear forms from $\boldsymbol{k}[\boldsymbol{T}]$ and $\boldsymbol{k}[\boldsymbol{u}]$, and which satisfy $\boldsymbol{T} \varphi_{\natural}=\left[\begin{array}{lll}Q_{1} & \cdots & Q_{\mu}\end{array}\right] C_{\natural}^{\prime}$ and $C_{\natural}^{\prime} \boldsymbol{u}^{\mathrm{T}}=A_{\natural}^{\prime} \boldsymbol{T}^{\mathrm{T}}$, for $\mu=\mu\left(I_{1}\left(\varphi_{\natural}\right)\right)$. The set of linearly independent forms $Q_{1}, \ldots, Q_{\mu}$ from $B_{c}$ may be extended to a basis $Q_{1}, \ldots, Q_{c+1}$ for $B_{c}$. The entries of $\rho^{(c)}$ also form a basis for $B_{c}$; so there is an invertible matrix $v$, with entries in $\boldsymbol{k}$, so that $\rho^{(c)}=\left[Q_{1}, \ldots, Q_{c+1}\right] v$. The matrix $C_{\natural}$ has been defined to satisfy $\boldsymbol{T} \varphi_{\natural}=\rho^{(c)} C_{\natural}$. Thus,

$$
\begin{aligned}
{\left[\begin{array}{lll}
Q_{1} & \cdots & Q_{c+1}
\end{array}\right] v C_{\natural} } & =\rho^{(c)} C_{\natural}=\boldsymbol{T} \varphi_{\natural}=\left[\begin{array}{lll}
Q_{1} & \cdots & Q_{\mu}
\end{array}\right] C_{\natural}^{\prime} \\
& =\left[\begin{array}{lll}
Q_{1} & \cdots & Q_{c+1}
\end{array}\right]\left[\begin{array}{c}
I_{\mu} \\
0
\end{array}\right] C_{\natural}^{\prime} ;
\end{aligned}
$$

hence, the matrices $v C_{\natural}$ and $\left[\begin{array}{c}I_{\mu} \\ 0\end{array}\right] C_{\natural}^{\prime}$, of linear forms from $k[T]$, are equal, and $C_{\natural}^{\prime}$ is obtained from $C_{\natural}$ by applying invertible row operations and suppressing zero rows. It quickly follows that $v A_{\natural}=\left[\begin{array}{c}I_{\mu} \\ 0\end{array}\right] A_{\natural}^{\prime}$, and $A_{\natural}^{\prime}$ is obtained from $A_{\natural}$ by applying invertible row operations and suppressing zero rows. This completes the proof of (1). To prove (2), one quickly calculates $\mu\left(I_{2}\left(C_{\natural}^{\prime}\right)\right)$ for each matrix $C_{\natural}^{\prime}$ of assertion 
(1). It is clear that $I_{2}\left(C_{\natural}^{\prime}\right)=I_{2}\left(C_{\natural}\right)$. One also calculates $\operatorname{gcd} I_{3}\left(A_{\natural}^{\prime}\right)=\operatorname{gcd} I_{3}\left(A_{\natural}\right)$ :

$\begin{array}{cc}\natural & \operatorname{gcd} I_{3}\left(A_{\natural}^{\prime}\right) \\ \left(\emptyset, \mu_{6}\right) & 1 \\ \left(\emptyset, \mu_{5}\right) & 1 \\ \left(c, \mu_{5}\right) & u_{2} \\ \left(\emptyset, \mu_{4}\right) & 1 \\ \left(c, \mu_{4}\right) & u_{2} \\ c, c & u_{2}\left(u_{1}+u_{2}\right) .\end{array}$

$\begin{array}{cc}\natural & \operatorname{gcd} I_{3}\left(A_{\natural}^{\prime}\right) \\ c: c & u_{2}^{2} \\ c: c: c & u_{2}^{3} \\ c: c, c & u_{1} u_{2}^{2} \\ c, c, c & \left(u_{1}+u_{2}\right) u_{1} u_{2} \\ \mu_{2} & 0\end{array}$

Remark 3.21 shows that the transformation $A_{\natural}$ to $A_{g^{-1}}$, for $g \in G$, replaces $u_{1}$ and $u_{2}$ with linearly independent linear forms $\ell_{1}$ and $\ell_{2}$ from $\boldsymbol{k}[\boldsymbol{u}]$. For (3), Theorem 3.22 guarantees that there are exactly $6-\mu\left(I_{2}\left(C_{\natural}\right)\right)$ distinct singularities of multiplicity $c$ on or infinitely near $\mathcal{C}_{\mathfrak{t}}$. We use Corollary 2.5 to identify these singularities.

The following small calculation provides a sufficient condition for the existence of a generalized zero in a matrix $\varphi$. We use this calculation three times as we complete our classification of the matrices $\varphi$ of Lemma 4.10. The most important application of this calculation occurs when the parameters " $b$ " and " $N$ " are both taken to be zero. In this case, the matrix " $\varphi$ " looks like $\left[\begin{array}{ll}A_{1} & A_{2}\end{array}\right]$.

Observation 4.12. Let $R$ be an algebra over the field $\boldsymbol{k}$ and

$$
\varphi=\left[\begin{array}{lll}
A_{1} & A_{2} & A_{3} \\
A_{4} & A_{5} & A_{6}
\end{array}\right]
$$

be a matrix with entries in $R$, where $A_{1}$ and $A_{2}$ are $a \times 1$ matrices, $A_{3}$ is an $a \times N$ matrix, $A_{4}$ and $A_{5}$ are $b \times 1$ matrices, and $A_{6}$ is an $b \times N$ matrix for some nonnegative integers $a, b$, and $N$. Suppose that every polynomial of degree a in $\boldsymbol{k}[x]$ has a root in $k$. Suppose further that each entry of $A_{2}$ is in the vector space spanned by the entries of $A_{1}$. Then there exist invertible matrices $\chi^{\prime}, \chi$, and $\xi$, with entries in $k$, such that

$$
\chi \varphi \xi=\left[\begin{array}{ccc}
A_{1}^{\prime} & A_{2}^{\prime \prime} & A_{3}^{\prime} \\
A_{4} & A_{5}^{\prime \prime} & A_{6}
\end{array}\right],
$$

where at least one entry of $A_{2}^{\prime \prime}$ is zero, $A_{i}^{\prime}=\chi^{\prime} A_{i}$ for $i$ equal to 1 or $3, A_{2}^{\prime \prime}=$ $\chi^{\prime}\left(A_{2}-\lambda A_{1}\right)$ and $A_{5}^{\prime \prime}=A_{5}-\lambda A_{4}$ for some $\lambda$ in $k$, and the submatrices $A_{4}$ and $A_{6}$ remain unchanged in the transformation from $\varphi$ to $\chi \varphi \xi$.

Proof. The hypothesis about the entries of $A_{1}$ and $A_{2}$ guarantees the existence of a matrix $M$, with entries in $k$, such that $A_{2}=M A_{1}$. The hypothesis about roots of polynomials ensures that $M$ has an eigenvalue $\lambda$ in $\boldsymbol{k}$. Let $\xi$ be the elementary 
matrix which subtracts $\lambda$ times column one from column two. The second column of $\varphi \xi$ is

$$
\left[\begin{array}{c}
(M-\lambda I) A_{1} \\
A_{5}-\lambda A_{4}
\end{array}\right]
$$

the other columns of $\varphi$ are unperturbed under the transformation $\varphi \mapsto \varphi \xi$. The matrix $M-\lambda I$ is singular, so there exists a non-zero row vector $v$, with entries in $k$, so that $v(M-\lambda I)=0$. Insert $v$ as a row in an invertible $a \times a$ matrix $\chi^{\prime}$ and let

$$
\chi=\left[\begin{array}{cc}
\chi^{\prime} & 0_{a \times b} \\
0_{b \times a} & I_{b \times b}
\end{array}\right]
$$

where 0 is a zero matrix and $I$ is an identity matrix. The triple $\left(\chi^{\prime}, \chi, \xi\right)$ satisfies the required properties.

Lemma 4.13. Let $\boldsymbol{k}$ be a field. Assume that every quadratic polynomial in $\boldsymbol{k}[x]$ has a root in $\boldsymbol{k}$. Let $R$ be a $\boldsymbol{k}$-algebra, and $\varphi$ be a $3 \times 2$ matrix with entries from $R$. Assume that the entries of $\varphi$ span a vector space of dimension 4 and ht $I_{2}(\varphi)=2$. Then there exist invertible matrices $\chi$ and $\xi$ over $k$ so that $\chi \varphi \xi$ has one of the following forms:

$$
\varphi_{\left(\emptyset, \mu_{4}\right)}=\left[\begin{array}{ll}
Q_{1} & Q_{2} \\
Q_{2} & Q_{3} \\
Q_{3} & Q_{4}
\end{array}\right], \quad \varphi_{\left(c, \mu_{4}\right)}=\left[\begin{array}{cc}
Q_{1} & Q_{2} \\
Q_{3} & Q_{1} \\
0 & Q_{4}
\end{array}\right], \quad \varphi_{c, c}=\left[\begin{array}{cc}
Q_{1} & Q_{2} \\
Q_{3} & Q_{3} \\
0 & Q_{4}
\end{array}\right], \quad \text { or } \quad \varphi_{c: c}=\left[\begin{array}{cc}
Q_{1} & Q_{3} \\
Q_{2} & Q_{4} \\
0 & Q_{2}
\end{array}\right]
$$

with $Q_{1}, Q_{2}, Q_{3}, Q_{4}$ linearly independent.

Proof. There are two possibilities for the original matrix $\varphi$. In Case 1, the entries in each column of $\varphi$ span a vector space of dimension 2. In Case 2, the entries of at least one of the columns of $\varphi$ span a vector space of dimension 3. In Case 1, $\varphi$ can be put in the form

$$
\left[\begin{array}{cc}
Q_{1} & *_{1} \\
Q_{2} & *_{2} \\
0 & *_{3}
\end{array}\right]
$$

where $Q_{1}, Q_{2}$ are linearly independent and $*_{1}, *_{2}$, and $*_{3}$ span a two dimensional subspace of $R$ which meets the vector space $\left\langle Q_{1}, Q_{2}>\right.$ only at 0 . The grade of $I_{2}(\varphi)$ is two; so no row of $\varphi$ can be zero. In particular, $*_{3}$ is not zero. We call $*_{3}$ by the name $Q_{3}$ and we have $Q_{1}, Q_{2}, Q_{3}$ linearly independent. At least one of the entries $*_{1}$ or $*_{2}$ is not in $<Q_{1}, Q_{2}, Q_{3}>$. Apply a row exchange and rename $Q_{1}$ and $Q_{2}$, if necessary. We have transformed $\varphi$ into the form

$$
\left[\begin{array}{cc}
Q_{1} & \alpha_{3} Q_{3}+\alpha_{4} Q_{4} \\
Q_{2} & Q_{4} \\
0 & Q_{3}
\end{array}\right]
$$


with $Q_{1}, Q_{2}, Q_{3}, Q_{4}$ linearly independent and $\alpha_{3}, \alpha_{4}$ in $k$. Let $\operatorname{Ro}_{i}$ and $\mathrm{Co}_{i}$ represent row $i$ and column $i$, respectively. Subtract $\alpha_{4} \mathrm{Ro}_{2}+\alpha_{3} \mathrm{Ro}_{3}$ from $\mathrm{Ro}_{1}$ and rename $Q_{1}-\alpha_{4} Q_{2}$ as $Q_{1}$. We have transformed $\varphi$ into

$$
\varphi^{\prime}=\left[\begin{array}{cc}
Q_{1} & 0 \\
Q_{2} & Q_{4} \\
0 & Q_{3}
\end{array}\right]
$$

which can be transformed into the form of $\varphi_{c, c}$.

In Case 2, the matrix $\varphi$ may be put in the form

$$
\left[\begin{array}{cc}
Q_{1} & \alpha_{1} Q_{1}+\alpha_{2} Q_{2}+\alpha_{3} Q_{3}+\alpha_{4} Q_{4} \\
Q_{2} & \beta_{1} Q_{1}+\beta_{2} Q_{2}+\beta_{3} Q_{3}+\beta_{4} Q_{4} \\
Q_{3} & Q_{4}
\end{array}\right]
$$

for some constants $\alpha_{i}, \beta_{i}$ with $Q_{1}, Q_{2}, Q_{3}, Q_{4}$ linearly independent. Subtract $\alpha_{4} \mathrm{Ro}_{4}$ from $\mathrm{Ro}_{1}$ and $\beta_{4} \mathrm{Ro}_{3}$ from $\operatorname{Ro}_{2}$. Rename $Q_{1}$ and $Q_{2}$ : the old $Q_{1}-\alpha_{4} Q_{3}$ becomes the new $Q_{1}$ and the old $Q_{2}-\beta_{4} Q_{3}$ becomes the new $Q_{2}$. Rename the constants $\alpha_{i}$ and $\beta_{i}$. We have transformed $\varphi$ into

$$
\left[\begin{array}{cc}
Q_{1} & \alpha_{1} Q_{1}+\alpha_{2} Q_{2}+\alpha_{3} Q_{3} \\
Q_{2} & \beta_{1} Q_{1}+\beta_{2} Q_{2}+\beta_{3} Q_{3} \\
Q_{3} & Q_{4}
\end{array}\right]
$$

Subtract $\alpha_{1} \mathrm{Co}_{1}$ from $\mathrm{Co}_{2}$ and rename $Q_{4}$ and $\beta_{2}$. The matrix $\varphi$ has become

$$
\varphi^{\prime}=\left[\begin{array}{cc}
Q_{1} & \alpha_{2} Q_{2}+\alpha_{3} Q_{3} \\
Q_{2} & \beta_{1} Q_{1}+\beta_{2} Q_{2}+\beta_{3} Q_{3} \\
Q_{3} & Q_{4}
\end{array}\right]
$$

At this point there are three cases. Either $\alpha_{3}=\beta_{3}=0$ (Case $2 \mathrm{~A}$ ), or $\alpha_{3} \neq 0$ (Case $2 \mathrm{~B}$ ), or $\alpha_{3}=0$ and $\beta_{3} \neq 0$ (case $2 \mathrm{C}$ ).

In case $2 \mathrm{~A}$, apply Observation 4.12 with

$$
\begin{array}{ll}
A_{1}=\left[\begin{array}{l}
Q_{1} \\
Q_{2}
\end{array}\right] & A_{2}=\left[\begin{array}{c}
\alpha_{2} Q_{2} \\
\beta_{1} Q_{1}+\beta_{2} Q_{2}
\end{array}\right] \\
A_{4}=\left[Q_{3}\right] & A_{5}=\left[Q_{4}\right]
\end{array}
$$

and transform $\varphi^{\prime}$ into

$$
\left[\begin{array}{cc}
Q_{1}^{\prime} & 0 \\
Q_{2}^{\prime} & * \\
Q_{3} & Q_{4}^{\prime}
\end{array}\right]
$$


where $Q_{1}^{\prime}, Q_{2}^{\prime}, Q_{3}, Q_{4}^{\prime}$ are linearly independent and $*$ is a non-zero element of the vector space $\left\langle Q_{1}^{\prime}, Q_{2}^{\prime}\right\rangle$. If $\left.* \in<Q_{1}^{\prime}\right\rangle$, then $\varphi$ may be transformed into $\varphi_{c: c}$; otherwise, $\varphi$ may be transformed into $\varphi_{c, c}$.

In Case 2B, one may quickly transform $\alpha_{3}$ into 1 . (Indeed, one may multiply $\mathrm{Co}_{2}$ by $\alpha_{3}^{-1}$ and rename $Q_{4}$ and the constants $\alpha_{i}$ and $\beta_{i}$.) At this point, $\varphi^{\prime}$ is

$$
\left[\begin{array}{cc}
Q_{1} & \alpha_{2} Q_{2}+Q_{3} \\
Q_{2} & \beta_{1} Q_{1}+\beta_{2} Q_{2}+\beta_{3} Q_{3} \\
Q_{3} & Q_{4}
\end{array}\right] .
$$

Add $\alpha_{2} \operatorname{Ro}_{2}$ to $\operatorname{Ro}_{3}$ and rename $Q_{3}, Q_{4}$, and the $\beta$ 's to obtain

$$
\left[\begin{array}{cc}
Q_{1} & Q_{3} \\
Q_{2} & \beta_{1} Q_{1}+\beta_{2} Q_{2}+\beta_{3} Q_{3} \\
Q_{3} & Q_{4}
\end{array}\right] .
$$

Subtract $\beta_{3} \mathrm{Ro}_{1}$ from $\mathrm{Ro}_{2}$ and rename $Q_{2}$ to obtain

$$
\left[\begin{array}{cc}
Q_{1} & Q_{3} \\
Q_{2} & \beta_{1} Q_{1}+\beta_{2} Q_{2} \\
Q_{3} & Q_{4}
\end{array}\right] .
$$

Subtract $\beta_{2} \mathrm{Co}_{1}$ from $\mathrm{Co}_{2}, \beta_{2} \mathrm{Ro}_{1}$ from $\mathrm{Ro}_{3}$, and rename $Q_{3}$ and $Q_{4}$ to obtain

$$
\left[\begin{array}{cc}
Q_{1} & Q_{3} \\
Q_{2} & \beta_{1} Q_{1} \\
Q_{3} & Q_{4}
\end{array}\right]
$$

If $\beta_{1}$ is zero, then $\varphi^{\prime}$ may be transformed into $\varphi_{\left(c, \mu_{4}\right)}$. If $\beta_{1}$ is not zero, then $\beta_{1}$ may be transformed into 1 . (One multiplies $\mathrm{Ro}_{2}$ by $\beta_{1}^{-1}$ and renames $Q_{2}$.) At this point one uses row and column exchanges to transform $\varphi^{\prime}$ into the form of $\varphi\left(\emptyset, \mu_{4}\right)$.

In Case 2C, one starts with

$$
\varphi^{\prime}=\left[\begin{array}{cc}
Q_{1} & \alpha_{2} Q_{2} \\
Q_{2} & \beta_{1} Q_{1}+\beta_{2} Q_{2}+\beta_{3} Q_{3} \\
Q_{3} & Q_{4}
\end{array}\right],
$$

with $\beta_{3} \neq 0$. Transform $\beta_{3}$ into 1 by multiplying $\mathrm{Co}_{2}$ by $\beta_{3}^{-1}$ and renaming $Q_{4}$ and the constants. Add $\beta_{1} \operatorname{Ro}_{1}+\beta_{2} \operatorname{Ro}_{2}$ to $\operatorname{Ro}_{3}$ and rename $Q_{3}$ and $Q_{4}$. The matrix $\varphi^{\prime}$ has become

$$
\left[\begin{array}{cc}
Q_{1} & \alpha_{2} Q_{2} \\
Q_{2} & Q_{3} \\
Q_{3} & Q_{4}
\end{array}\right]
$$

If $\alpha_{2}=0$, then $\varphi^{\prime}$ may be transformed into the matrix $\varphi_{\left(c, \mu_{4}\right)}$. If $\alpha_{2} \neq 0$, then $\alpha_{2}$ may be transformed into 1 (by multiplying $\operatorname{Ro}_{1}$ by $\alpha_{2}^{-1}$ and renaming $Q_{1}$ ) and $\varphi^{\prime}$ may be transformed into the form of $\varphi\left(\emptyset, \mu_{4}\right)$. 
Lemma 4.14. Let $\boldsymbol{k}$ be a field. Assume that every polynomial in $\boldsymbol{k}[x]$ of degree 2 or 3 has a root in $\boldsymbol{k}$. Let $R$ be a $\boldsymbol{k}$-algebra, and $\varphi$ be a $3 \times 2$ matrix with entries from $R$. Assume that the entries of $\varphi$ span a vector space of dimension 3 and ht $I_{2}(\varphi)=2$. Then there exist invertible matrices $\chi$ and $\xi$ over $\boldsymbol{k}$ so that $\chi \varphi \xi$ has one of the following forms:

$$
\varphi_{c: c: c}=\left[\begin{array}{cc}
Q_{1} & Q_{2} \\
Q_{3} & Q_{1} \\
0 & Q_{3}
\end{array}\right], \quad \varphi_{c: c, c}=\left[\begin{array}{cc}
Q_{1} & 0 \\
Q_{2} & Q_{3} \\
0 & Q_{2}
\end{array}\right], \quad \text { or } \quad \varphi_{c, c, c}=\left[\begin{array}{cc}
Q_{1} & Q_{1} \\
Q_{2} & 0 \\
0 & Q_{3}
\end{array}\right]
$$

with $Q_{1}, Q_{2}, Q_{3}$ linearly independent.

Proof. First we show that there exist invertible matrices $\chi$ and $\xi$ so that some entry of $\chi \varphi \xi$ is zero. There is nothing to show unless the entries in the first column of $\varphi$ are linearly independent; so, we make this assumption. The hypothesis that the entries of $\varphi$ span a vector space of dimension 3 tells us that every entry in column two of $\varphi$ is contained in the vector space spanned by the entries of column one of $\varphi$. Apply Observation 4.12 to transform $\varphi$ into a matrix which contains a zero. Further elementary row and column operations put $\varphi$ into one of the forms

$$
\left[\begin{array}{cc}
Q_{1} & * \\
Q_{2} & * \\
0 & Q_{3}
\end{array}\right] \quad \text { or } \quad\left[\begin{array}{cc}
Q_{1} & * \\
Q_{2} & * \\
0 & Q_{1}
\end{array}\right]
$$

with $Q_{1}, Q_{2}, Q_{3}$ linearly independent.

We first work on the left hand case of (4.15). Apply elementary row operations in order to make the entries labeled $*$ be in the vector space $\left\langle Q_{1}, Q_{2}\right\rangle$. Now we apply Observation 4.12 with

$$
\begin{array}{ll}
A_{1}=\left[\begin{array}{l}
Q_{1} \\
Q_{2}
\end{array}\right] & A_{2}=\left[\begin{array}{l}
* \\
*
\end{array}\right] \\
A_{4}=[0] & A_{5}=\left[Q_{3}\right]
\end{array}
$$

and transform $\varphi$ into the form

$$
\left[\begin{array}{cc}
Q_{1} & a Q_{1}+b Q_{2} \\
Q_{2} & 0 \\
0 & Q_{3}
\end{array}\right]
$$

(It might be necessary to re-name $Q_{1}$ and $Q_{2}$.) There are two cases.

We first consider the case $a \neq 0$. Add $b / a$ times row 2 to row 1 , multiply column 1 by $a$, and rename $Q_{1}$ to obtain a matrix of the form of $\varphi_{c, c, c}$. 
Now we consider the case $a=0$ in (4.16). In this case the hypothesis that ht $I_{2}(\varphi)=2$ ensures that $b \neq 0$. It is easy to transform $\varphi$ into a matrix with the form $\varphi_{c: c, c}$.

Finally, we consider the matrix on the right side of (4.15). If the entry in position $(2,1)$ is in the vector space $\left\langle Q_{1}, Q_{2}>\right.$, we may use elementary row operations to put $\varphi$ into the form

$$
\left[\begin{array}{cc}
Q_{1} & a Q_{2} \\
Q_{2} & Q_{3} \\
0 & Q_{1}
\end{array}\right]
$$

The constant $a$ can not be zero because $h t\left(I_{2}(\varphi)\right)=2$ and $\varphi$ may be transformed into the form of the matrix $\varphi_{c: c: c}$. On the other hand, if the $(2,1)$ entry of $\varphi$ is not in $\left.<Q_{1}, Q_{2}\right\rangle$, then $\varphi$ may be transformed into a matrix of the form

$$
\left[\begin{array}{cc}
Q_{1} & Q_{3} \\
Q_{2} & * \\
0 & Q_{1}
\end{array}\right]
$$

Use a column operation and rename $Q_{3}$ to put $*$ into $<Q_{1}, Q_{3}>$. Use row operations, and rename $Q_{2}$, to put $\varphi$ into the form

$$
\left[\begin{array}{cc}
Q_{1} & Q_{3} \\
Q_{2} & 0 \\
0 & Q_{1}
\end{array}\right]
$$

This matrix may be easily transformed into a matrix of the form $\varphi_{c: c, c}$.

When we decompose $\mathbb{T}_{d}$ into strata in Section 6 , most of our calculations (in particular the verification of irreducibility as well as the calculation of dimension) are made using the closure of a given stratum rather than the stratum itself. To facilitate those calculations, we gather the disjoint orbits $\mathrm{DO}_{\natural}$ for $\downarrow \in \mathrm{ECP}$, with $\natural<\#$ together to form the combined orbit $\mathrm{CO}_{\#}$ for $\# \in \mathrm{CP}$. Our proofs in Section 6 require that we identify a well understood irreducible variety $N_{\#}$ with $\mathrm{CO}_{\#}=G \cdot N_{\#}$. We lay out our candidates for $N_{\#}$ in Definition 4.17 and show that our candidates have the relevant properties in Theorem 4.18.

Definition 4.17. (1) For each \# $\in \mathrm{CP}$, define $\mathrm{CO}_{\#}^{\text {Bal }}$ to be the subset

$$
\mathrm{CO}_{\#}^{\text {Bal }}=\bigcup_{\{\natural \in \mathrm{ECP} \mid \natural \leq \# \text { in TCP }\}} \mathrm{DO}_{\sharp}^{\text {Bal }}
$$

of $\mathrm{BalH}_{d}$. 
(2) For each \# $\in \mathrm{CP}$, define $N_{\#}^{\mathrm{Bal}}$ to be the following subset of $\mathrm{BalH}_{d}$ :

$$
\begin{aligned}
& N_{c: c: c}^{\mathrm{Bal}}=\left\{\left[\begin{array}{cc}
Q_{1} & Q_{2} \\
Q_{3} & Q_{1} \\
0 & Q_{3}
\end{array}\right] \in \mathrm{BalH}_{d}\right\} \\
& N_{c: c, c}^{\mathrm{Bal}}=\left\{\left[\begin{array}{cc}
Q_{1} & Q_{2} \\
Q_{3} & Q_{4} \\
0 & Q_{3}
\end{array}\right] \in \mathrm{BalH}_{d} \mid \operatorname{dim}_{\boldsymbol{k}}<Q_{1}, Q_{2}, Q_{3}, Q_{4}>\leq 3\right\} \text {, } \\
& N_{c, c, c}^{\mathrm{Bal}}=\left\{\left[\begin{array}{cc}
Q_{1} & Q_{2} \\
Q_{3} & Q_{4} \\
0 & Q_{5}
\end{array}\right] \in \mathrm{BalH}_{d} \mid \operatorname{dim}_{\boldsymbol{k}}<Q_{1}, Q_{2}, Q_{3}, Q_{4}, Q_{5}>\leq 3\right\} \text {, } \\
& N_{c: c}^{\mathrm{Bal}}=\left\{\left[\begin{array}{cc}
Q_{1} & Q_{2} \\
Q_{3} & Q_{4} \\
0 & Q_{3}
\end{array}\right] \in \mathrm{BalH}_{d}\right\} \\
& N_{c, c}^{\mathrm{Bal}}=\left\{\left[\begin{array}{cc}
Q_{1} & Q_{2} \\
Q_{3} & Q_{4} \\
0 & Q_{5}
\end{array}\right] \in \mathrm{BalH}_{d} \mid \operatorname{dim}_{\boldsymbol{k}}<Q_{3}, Q_{4}, Q_{5}>\leq 2\right\}, \\
& N_{c}^{\mathrm{Bal}}=\left\{\left[\begin{array}{cc}
Q_{1} & Q_{2} \\
Q_{3} & Q_{4} \\
0 & Q_{5}
\end{array}\right] \in \mathrm{BalH}_{d}\right\}, \text { and } \\
& N_{\emptyset}^{\mathrm{Bal}}=\mathrm{BalH}_{d} \text {. }
\end{aligned}
$$

(3) For each $\# \in \mathrm{CP}$, let $\mathrm{CO}_{\#}=\mathrm{CO}_{\#}^{\mathrm{Bal}} \cap \mathbb{B H}_{d}$ and $N_{\#}=N_{\#}^{\mathrm{Bal}} \cap \mathbb{B H}_{d}$.

Theorem 4.18. Let $\boldsymbol{k}$ be a field which satisfies the hypothesis of Theorem 4.9.

(1) If $\natural \in \mathrm{ECP}$ and $\# \in \mathrm{CP}$, with $\emptyset \leq \#$ in $\mathrm{TCP}$, then $M_{\natural}^{\mathrm{Bal}} \subseteq N_{\#}^{\mathrm{Bal}}$ and $M_{\natural} \subseteq N_{\#}$.

(2) If \# $\in \mathrm{CP}$, then $\mathrm{CO}_{\#}^{\mathrm{Bal}}=G \cdot N_{\#}^{\mathrm{Bal}}$ and $\mathrm{CO}_{\#}=G \cdot N_{\#}$

(3) The varieties $N_{\#}^{\mathrm{Bal}}$ and $N_{\#}$ are irreducible for all \# $\in \mathrm{CP}$.

Remark. The definition of $\mathrm{CO}_{\#}^{\mathrm{Bal}}$ ensures that

$$
\#^{\prime}<\# \text { in } \mathrm{CP} \Longrightarrow \mathrm{CO}_{\#^{\prime}}^{\mathrm{Bal}} \subseteq \mathrm{CO}_{\#}^{\mathrm{Bal}}
$$

However,

$$
\#^{\prime}<\# \text { in } \mathrm{CP} \text { does not imply } N_{\#^{\prime}}^{\mathrm{Bal}} \subseteq N_{\#}^{\mathrm{Bal}}
$$


Indeed, $\{c, c, c\}<\{c, c\}$ and if

$$
\varphi=\left[\begin{array}{cc}
Q_{1} & Q_{2} \\
Q_{2} & Q_{3} \\
0 & Q_{1}
\end{array}\right]
$$

with $Q_{1}, Q_{2}, Q_{3}$ linearly independent elements of $B_{c}$ and $Q_{1}, Q_{2}$ relatively prime, then $\varphi \in N_{c, c, c}^{\mathrm{Bal}}$ and $\varphi \notin N_{c, c}^{\mathrm{Bal}}$.

Proof. It suffices to establish the assertions in $\mathrm{BalH}_{d}$. One may then intersect with $\mathbb{B H}_{d}$ to obtain the comparable result in $\mathbb{B H}_{d}$. To establish (1), one must verify many inclusions; but each inclusion is completely straightforward. We prove (2). The inclusion $\mathrm{CO}_{\#}^{\mathrm{Bal}} \subseteq G \cdot N_{\#}^{\mathrm{Bal}}$ follows from (1). We now prove $G \cdot N_{\#}^{\mathrm{Bal}} \subseteq \mathrm{CO}_{\#}^{\mathrm{Bal}}$. Fix $\varphi \in N_{\#}^{\mathrm{Bal}}$ for some $\# \in \mathrm{CP}$. We prove that there exists $\downarrow \in \mathrm{ECP}$ with $\downarrow \leq \#$ and $\varphi \in \mathrm{DO}_{\natural}^{\mathrm{Bal}}$.

If \# is $c: c: c$, then either $\mu\left(I_{1}(\varphi)\right)=3$ and $\varphi$ is already in $M_{c: c: c}^{\mathrm{Bal}}$ or $\mu\left(I_{1}(\varphi)\right)=2$ and $\varphi \in \mathrm{DO}_{\mu_{2}}^{\mathrm{Bal}}$ by the proof of Theorem 4.9 .

If \# is $c: c, c$, then

$$
\varphi=\left[\begin{array}{cc}
Q_{1} & * \\
Q_{3} & * \\
0 & Q_{3}
\end{array}\right]
$$

with $Q_{1}, Q_{3}$ linearly independent and $\mu\left(I_{1}(\varphi)\right) \leq 3$. If $\mu\left(I_{1}(\varphi)\right)=2$, then $\varphi$ is in $\mathrm{DO}_{\mu_{2}}^{\mathrm{Bal}}$, as above. If $\mu\left(I_{1}(\varphi)\right)=3$, then $\varphi$ appears on the right side of (4.15) and the proof of Lemma 4.14 shows that $\varphi$ is in $\mathrm{DO}_{c: c: c}$ or $\mathrm{DO}_{c: c, c}$.

If \# is $c, c, c$, then $\mu\left(I_{1}(\varphi)\right) \leq 3$ and Theorem 4.9, together with the chart of (2) in Lemma 4.10 shows that $\varphi \in \mathrm{DO}_{\natural}^{\text {Bal }}$ for $\downarrow$ equal to $\mu_{2},\{c: c: c\},\{c: c, c\}$, or $\{c, c, c\}$. In any event, $\downarrow \leq\{c, c, c\}$.

If \# is $c: c$, then either $\mu\left(I_{1}(\varphi)\right)=4$ and $\varphi$ is in $M_{c: c}^{\mathrm{Bal}}$; or else, $\mu\left(I_{1}(\varphi)\right) \leq 3$ and $\varphi \in N_{c: c, c}^{\mathrm{Bal}}$. We have already shown that $N_{c: c, c}^{\mathrm{Bal}} \subseteq \mathrm{CO}_{c: c, c}^{\mathrm{Bal}}$. The definition of $\mathrm{CO}_{\#}^{\mathrm{Bal}}$ shows $\mathrm{CO}_{c: c, c}^{\mathrm{Bal}} \subseteq \mathrm{CO}_{c: c}^{\mathrm{Bal}}$, since $\{c: c, c\}<\{c: c\}$.

If $\#$ is $c, c$, then

$$
\varphi=\left[\begin{array}{cc}
Q_{1} & Q_{2} \\
Q_{3} & Q_{4} \\
0 & Q_{5}
\end{array}\right]
$$

with $\operatorname{dim}_{k}<Q_{3}, Q_{4}, Q_{5}>\leq 2$. If $\mu\left(I_{1}(\varphi)\right) \leq 3$, then $\varphi \in N_{c, c, c}^{\mathrm{Bal}} \subseteq \mathrm{CO}_{c, c, c}^{\mathrm{Bal}} \subseteq \mathrm{CO}_{c, c}^{\mathrm{Bal}}$. Henceforth, we assume $\mu\left(I_{1}(\varphi)\right)=4$. We look at the proof of Lemma 4.13. If Case 1 is in effect, then $\varphi \in \mathrm{DO}_{c, c}^{\mathrm{Bal}}$. We assume that Case 2 is in effect. This forces $Q_{2}, Q_{4}, Q_{5}$ to be linearly independent. The hypothesis $\operatorname{dim}_{k}<Q_{3}, Q_{4}, Q_{5}>\leq 2$ may be re-written as $Q_{3} \in<Q_{4}, Q_{5}>$. The hypothesis $\mu\left(I_{1}(\varphi)\right)=4$ now forces 
$Q_{1}, Q_{2}, Q_{4}, Q_{5}$ to be linearly independent. We permute the rows and columns of $\varphi$ to obtain

$$
g \varphi=\left[\begin{array}{ll}
Q_{5} & 0 \\
Q_{4} & Q_{3} \\
Q_{2} & Q_{1}
\end{array}\right],
$$

with $Q_{3}=\beta_{1} Q_{5}+\beta_{2} Q_{4}$. This is Case $2 \mathrm{~A}$ from the proof of Lemma 4.13. We conclude $\varphi \in \mathrm{DO}_{c, c}^{\mathrm{Bal}} \cup \mathrm{DO}_{c: c}^{\mathrm{Bal}}$.

Take \# to be $c$. If $\mu\left(I_{1}(\varphi)\right)=5$, then $\varphi \in M_{\left(c, \mu_{5}\right)}^{\mathrm{Bal}}$ and $\left(c, \mu_{5}\right)<c$. If $\mu\left(I_{1}(\varphi)\right) \leq$ 4 , then the chart in (2) of Lemma 4.10 shows that either $\varphi \in M_{\left(\emptyset, \mu_{4}\right)}^{\mathrm{Bal}}$; or else, $\varphi \in M_{\natural}^{\mathrm{Bal}}$ for some $\downarrow$ in ECP with $\downarrow<c$. On the other hand,

$$
M_{\left(\emptyset, \mu_{4}\right)}^{\mathrm{Bal}}=\left\{\theta \in \mathrm{BalH}_{d} \mid \mu\left(I_{1}(\theta)\right)=4 \text { and } \theta \text { does not have a generalized zero }\right\} .
$$

One entry of $\varphi$ is zero; so, $\varphi \notin M_{\left(\emptyset, \mu_{4}\right)}^{\mathrm{Bal}}$ and $\varphi$ is in $\mathrm{CO}_{c}^{\mathrm{Bal}}$. (One could also argue that $\varphi \notin M_{\left(\emptyset, \mu_{4}\right)}^{\mathrm{Bal}}$ because if $C$ is the companion to $\varphi$ in the sense of $(3.11)$, then $\mu\left(I_{2}(C)\right) \leq 5$ and this is too small for $\varphi$ to be in $M_{\left(\emptyset, \mu_{4}\right)}^{\mathrm{Bal}}$.)

Finally, if \# is $\emptyset$, then Theorem 4.9 shows that $\varphi \in \mathrm{DO}_{\natural}^{\mathrm{Bal}}$ for some $\downarrow \in$ ECP. This $\downarrow$ automatically satisfies $\downarrow<\emptyset$.

The proof of (2) is complete. For (3), notice that each set $N_{\#}^{\mathrm{Bal}}$ and $N_{\#}$ has the form $V \cap \mathrm{BalH}_{d}$ or $V \cap \mathbb{B H}_{d}$, where $V$ is a closed irreducible subset of the affine space $\mathbb{H}_{d}$. Indeed, for example, the condition $\operatorname{dim}_{\boldsymbol{k}}<Q_{3}, Q_{4}, Q_{5}>\leq 2$ is defined by the prime ideal generated by the maximal order minors of a generic $3 \times(c+1)$ matrix. Furthermore, $\mathrm{BalH}_{d}$ and $\mathbb{B H}_{d}$ are open subsets of $\mathbb{H}_{d}$, see Observation 5.29 .

Assume that the field $\boldsymbol{k}$ is infinite throughout the rest of the section. Proposition 4.21 shows that $\mathrm{DO}_{\natural}$ is non-empty whenever it has a chance of being non-empty. In other words, $\mathrm{DO}_{\mu_{2}}$ is always empty; and, if an element of $\mathrm{DO}_{\natural}$ requires more linearly independent entries than are available in $B_{c}$, then $\mathrm{DO}_{\natural}$ is empty. Otherwise, $\mathrm{DO}_{\natural}$ is non-empty. To prove Proposition 4.21 we must show that the signed maximal order minors of a particular $3 \times 2$ matrix determine a birational parameterization of a curve. Lemma 4.19 provides an explicit sufficient condition for establishing this birationality; recall that the spaces $\mathbb{H}_{d}$ and $\mathbb{B H}_{d}$ are defined in Definition 4.3. We use the Avoidance Lemma 4.20 repeatedly in the proof of Proposition 4.21.

Lemma 4.19. Let $\boldsymbol{k}$ be an infinite field and let $\varphi$ be an element of $\mathbb{H}_{d}$ with ht $I_{2}(\varphi)=2$ and $\mu\left(I_{1}(\varphi)\right) \geq 3$. If two of the entries of $\varphi$ are $x^{c}$ and $y^{c-1}(x+y)$, then $\varphi \in \mathbb{B H}_{d}$.

Proof. We must show that the morphism, $\Psi: \mathbb{P}^{1} \rightarrow \mathbb{P}^{2}$, given by the signed maximal order minors of $\varphi$, is birational. Let $\overline{\boldsymbol{k}}$ be the algebraic closure of $\boldsymbol{k}$. The field $\boldsymbol{k}$ 
is infinite; so $\Psi$ is birational if and only if the induced morphism $\Psi_{\overline{\boldsymbol{k}}}: \mathbb{P}_{\overline{\boldsymbol{k}}}^{1} \rightarrow \mathbb{P}_{\overline{\boldsymbol{k}}}^{2}$ is birational. Throughout the rest of this proof, we assume that $\boldsymbol{k}$ is algebraically closed.

In the language of $(4.5)$, write $\Phi(\varphi)$ as $\left(g_{1}, g_{2}, g_{3}\right)$. Adopt the notation of Theorem 0.10. In particular, let $r$ be the degree of the field extension

$$
r=\left[\text { Quot } \boldsymbol{k}\left[B_{d}\right]: \text { Quot } \boldsymbol{k}\left[g_{1}, g_{2}, g_{3}\right]\right],
$$

and $e$ be the multiplicity of the standard graded $\boldsymbol{k}$-algebra $\boldsymbol{k}\left[g_{1}, g_{2}, g_{3}\right]$. Theorem 0.10 guarantees that $r e=d$, and $\Psi$ is birational if and only if $r=1$. Furthermore, according to Theorem 0.10 and Observation 0.11 , there exist homogeneous forms $f_{1}$ and $f_{2}$ in $B$ of degree $r$ so that every entry of $\varphi$ is in $\boldsymbol{k}\left[f_{1}, f_{2}\right]$. Let

$$
\theta: k\left[z_{1}, z_{2}\right] \rightarrow \boldsymbol{k}\left[f_{1}, f_{2}\right]
$$

be the $\boldsymbol{k}$-algebra homomorphism with $\theta\left(z_{i}\right)=f_{i}$, as described in the proof of Observation 0.11. Select homogeneous forms $Q_{1}^{\prime}$ and $Q_{2}^{\prime}$ in $\boldsymbol{k}\left[z_{1}, z_{2}\right]$ with $\theta\left(Q_{1}^{\prime}\right)=x^{c}$ and $\theta\left(Q_{2}^{\prime}\right)=y^{c-1}(x+y)$. Let $\varepsilon$ be the degree of the $Q_{i}^{\prime}$. It follows that $c=\varepsilon r$. The equations

$$
e r=d=2 c=2 \varepsilon r
$$

yield $e=2 \varepsilon$. Write $Q_{i}^{\prime}=\prod_{j=1}^{\varepsilon} \ell_{i, j}$, for linear forms $\ell_{i, j}$ in $k\left[z_{1}, z_{2}\right]$. We notice that $\varepsilon \geq 2$. Indeed, if $\varepsilon$ were equal to 1 , then $r$ would equal $c$ and the entries of $\varphi$ would all live in the two-dimensional vector space $\left\langle f_{1}, f_{2}>\right.$ and this would violate the hypothesis that $\mu\left(I_{1}(\varphi)\right) \geq 3$. The equations

$$
x^{c}=\theta\left(Q_{1}^{\prime}\right)=\prod \ell_{1, j}\left(f_{1}, f_{2}\right) \quad \text { and } \quad y^{c-1}(x+y)=\theta\left(Q_{2}^{\prime}\right)=\prod \ell_{2, j}\left(f_{1}, f_{2}\right),
$$

which take place in the Unique Factorization Domain $B$, tell us that, after renumbering the linear factors and adjusting their constants, we have

$$
\begin{aligned}
x^{r} & =\ell_{1, j}\left(f_{1}, f_{2}\right), & & \text { for all } j, \\
y^{r-1}(x+y) & =\ell_{2,1}\left(f_{1}, f_{2}\right), & & \text { and } \\
y^{r} & =\ell_{2, j}\left(f_{1}, f_{2}\right), & & \text { for all } j \geq 2 .
\end{aligned}
$$

The linear forms $\ell_{1,1}, \ell_{2,1}$, and $\ell_{2,2}$ in $\boldsymbol{k}\left[z_{1}, z_{2}\right]$ look like

$$
\ell_{1,1}=\alpha z_{1}+\beta z_{2}, \quad \ell_{2,1}=\alpha^{\prime} z_{1}+\beta^{\prime} z_{2}, \quad \text { and } \quad \ell_{2,2}=\alpha^{\prime \prime} z_{1}+\beta^{\prime \prime} z_{2}
$$

for constants $\alpha, \beta, \alpha^{\prime}, \beta^{\prime}, \alpha^{\prime \prime}$, and $\beta^{\prime \prime}$ in $k$. It follows that

$$
\left[\begin{array}{l}
x^{r} \\
y^{r}
\end{array}\right]=\left[\begin{array}{cc}
\alpha & \beta \\
\alpha^{\prime \prime} & \beta^{\prime \prime}
\end{array}\right]\left[\begin{array}{l}
f_{1} \\
f_{2}
\end{array}\right] .
$$


The $2 \times 2$ matrix in the above equation is necessarily invertible. We also have that $y^{r-1}(x+y)$ is equal to

$$
\ell_{2,1}\left(f_{1}, f_{2}\right)=\left[\begin{array}{ll}
\alpha^{\prime} & \beta^{\prime}
\end{array}\right]\left[\begin{array}{l}
f_{1} \\
f_{2}
\end{array}\right]=\left[\begin{array}{ll}
\alpha^{\prime} & \beta^{\prime}
\end{array}\right]\left[\begin{array}{cc}
\alpha & \beta \\
\alpha^{\prime \prime} & \beta^{\prime \prime}
\end{array}\right]^{-1}\left[\begin{array}{l}
x^{r} \\
y^{r}
\end{array}\right]=a x^{r}+b y^{r},
$$

where $a$ and $b$ are the elements of $\boldsymbol{k}$ which are defined by

$$
\left[\begin{array}{ll}
a & b
\end{array}\right]=\left[\begin{array}{ll}
\alpha^{\prime} & \beta^{\prime}
\end{array}\right]\left[\begin{array}{cc}
\alpha & \beta \\
\alpha^{\prime \prime} & \beta^{\prime \prime}
\end{array}\right]^{-1} \text {. }
$$

The equation $y^{r-1}(x+y)=a x^{r}+b y^{r}$ is impossible in $k[x, y]$, unless $r=1$. Thus, $r=1, \Psi$ is birational, and the proof is complete.

Lemma 4.20. Let $B$ be the polynomial ring $B=\boldsymbol{k}[x, y]$, where $\boldsymbol{k}$ is an infinite field, and $c$ be a positive integer. If $V$ is proper subspace of $B_{c}$ and $f$ is a non-zero homogeneous polynomial in $B$, then there exists a polynomial $Q$ in $B_{c} \backslash V$ with $Q$ and $f$ relatively prime.

Proof. Let $\prod f_{i}$ be the factorization of $f$ into homogeneous irreducible factors in $B$. Each irreducible factor $f_{i}$ gives rise to the proper subspace $V_{i}=B_{c-\operatorname{deg} f_{i}} f_{i}$ of $B_{c}$. (If $\operatorname{deg} f_{i}>\operatorname{deg} Q$, then the vector space $V_{i}$ is automatically equal to zero.) The field $k$ is infinite; so, $V \cup \bigcup_{i} V_{i}$ is a proper subset of $B_{c}$. Any $Q$ in the complement of $V \cup \bigcup_{i} V_{i}$ has the desired property.

Proposition 4.21. Let $k$ be an infinite field and $d=2 c$ be an even integer. Fix $\natural \in \operatorname{ECP} \backslash\left\{\mu_{2}\right\}$.

(1) If $d=2$, then $\mathrm{DO}_{\natural}$ is empty.

(2) If $d=4$, then $\mathrm{DO}_{\natural}$ is non-empty if and only if $\natural \leq\{c, c, c\}$.

(3) If $d=6$, then $\mathrm{DO}_{\natural}$ is non-empty if and only if $\emptyset \leq\left(c, \mu_{4}\right)$ or $\downarrow=\left(\emptyset, \mu_{4}\right)$.

(4) If $d=8$, then $\mathrm{DO}_{\natural}$ is non-empty if and only if $\emptyset \leq\left(\emptyset, \mu_{5}\right)$ or $\downarrow=\left(\emptyset, \mu_{4}\right)$

(5) If $10 \leq d$, then $\mathrm{DO}_{\natural}$ is non-empty.

Proof. We first consider $\downarrow \in$ ECP with $\downarrow<\emptyset$. We show that there exist linearly independent $Q_{1}, Q_{2}, \ldots$ in $B_{c}$ so that $\varphi_{\natural}$ is in $\mathrm{DO}_{\natural}$, where

$$
\begin{aligned}
\varphi_{c: c: c}= & {\left[\begin{array}{ll}
Q_{1} & Q_{2} \\
Q_{3} & Q_{1} \\
0 & Q_{3}
\end{array}\right], \varphi_{c: c, c}=\left[\begin{array}{cc}
Q_{1} & 0 \\
Q_{2} & Q_{3} \\
0 & Q_{2}
\end{array}\right], \varphi_{c, c, c}=\left[\begin{array}{cc}
Q_{1} & Q_{1} \\
Q_{2} & 0 \\
0 & Q_{3}
\end{array}\right], } \\
\varphi_{c: c}= & {\left[\begin{array}{ll}
Q_{1} & Q_{2} \\
Q_{3} & Q_{4} \\
0 & Q_{3}
\end{array}\right], \varphi_{c, c}=\left[\begin{array}{ll}
Q_{1} & Q_{2} \\
Q_{3} & Q_{3} \\
0 & Q_{4}
\end{array}\right], \varphi_{\left(c, \mu_{4}\right)}=\left[\begin{array}{cc}
Q_{1} & Q_{2} \\
Q_{3} & Q_{1} \\
0 & Q_{4}
\end{array}\right], } \\
\varphi_{\left(c, \mu_{5}\right)}= & {\left[\begin{array}{ll}
Q_{1} & Q_{2} \\
Q_{3} & Q_{4} \\
0 & Q_{5}
\end{array}\right] . }
\end{aligned}
$$


For each $\downarrow$, take $Q_{1}=x^{c}$ and $Q_{2}=y^{c-1}(x+y)$. We show how to pick the rest of the $Q_{i}$. According to Lemma 4.19 we need only verify that $\operatorname{ht}\left(I_{2}\left(\varphi_{\natural}\right)\right)=2$. We apply the Avoidance Lemma 4.20 repeatedly.

Take $\downarrow$ to be $\{c: c: c\},\{c: c, c\}$, or $\{c, c, c\}$. Pick $Q_{3} \in B_{c}$ so that $Q_{3}$ is not in $<Q_{1}, Q_{2}>$ and $Q_{3}$ and $Q_{1} Q_{2}$ are relatively prime. Observe $\operatorname{ht}\left(I_{2}\left(\varphi_{\natural}\right)\right)=2$.

Take $\downarrow$ to be $\{c: c\}$. Pick $Q_{3} \in B_{c}$ so that $Q_{3} \notin<Q_{1}, Q_{2}>$ and $Q_{3}$ and $Q_{1}$ are relatively prime. Pick $Q_{4} \in B_{c}$ so that $Q_{4} \notin<Q_{1}, Q_{2}, Q_{3}>$ and $Q_{4}$ and $Q_{3}$ are relatively prime. Observe ht $\left(I_{2}\left(\varphi_{\natural}\right)\right)=2$.

Take $\downarrow$ to be $\{c, c\}$. Pick $Q_{3} \in B_{c}$ so $Q_{3} \notin<Q_{1}, Q_{2}>$ and $Q_{3}$ is relatively prime to $Q_{1}$. Pick $Q_{4} \in B_{c}$ so that $Q_{4} \notin<Q_{1}, Q_{2}, Q_{3}>$ and $Q_{4}$ is relatively prime to $Q_{3}\left(Q_{1}-Q_{2}\right)$. Observe ht $\left(I_{2}\left(\varphi_{\natural}\right)\right)=2$.

Take $\downarrow$ to be $\left(c, \mu_{4}\right)$. Pick $Q_{3} \in B_{c}$ so $Q_{3} \notin<Q_{1}, Q_{2}>$ and $Q_{3}$ is relatively prime to $Q_{1}$. Pick $Q_{4} \in B_{c}$ so that $Q_{4} \notin<Q_{1}, Q_{2}, Q_{3}>$ and $Q_{4}$ is relatively prime to $Q_{1}^{2}-Q_{2} Q_{3}$. Observe $\operatorname{ht}\left(I_{2}\left(\varphi_{\natural}\right)\right)=2$.

Take $\downarrow$ to be $\left(c, \mu_{5}\right)$. Pick $Q_{3} \in B_{c}$ so $Q_{3} \notin<Q_{1}, Q_{2}>$ and $Q_{3}$ is relatively prime to $Q_{1}$. Pick $Q_{4} \in B_{c}$ so that $Q_{4} \notin<Q_{1}, Q_{2}, Q_{3}>$ and $Q_{4}$ is relatively prime to $Q_{1} Q_{3}$. Pick $Q_{5} \in B_{c}$ so that $Q_{5} \notin<Q_{1}, Q_{2}, Q_{3}, Q_{4}>$ and $Q_{5}$ is relatively prime to $Q_{1}\left(Q_{1} Q_{4}-Q_{2} Q_{3}\right)$. Observe $\operatorname{ht}\left(I_{2}\left(\varphi_{\natural}\right)\right)=2$.

For the final three elements of ECP $\backslash\left\{\mathrm{DO}_{\mu_{2}}\right\}$ we verify directly that the matrix $\varphi_{\natural}$, given below, is in $\mathrm{DO}_{\natural}$ :

$$
\begin{aligned}
\varphi_{\left(\emptyset, \mu_{4}\right)} & =\left[\begin{array}{cc}
y x^{c-1} & x^{c} \\
x^{c} & y^{c} \\
y^{c} & y^{c-1}(x+y)
\end{array}\right], \quad \varphi_{\left(\emptyset, \mu_{5}\right)}=\left[\begin{array}{cc}
x^{c} & y^{c-2}\left(x^{2}+y^{2}\right) \\
y^{c} & y x^{c-1} \\
y^{c-1}(x+y) & x^{c}
\end{array}\right], \text { and } \\
\varphi_{\left(\emptyset, \mu_{6}\right)} & =\left[\begin{array}{cc}
x^{c-2}\left(x^{2}+y^{2}\right) & y^{c-2}\left(x^{2}+y^{2}\right) \\
y^{c} & y x^{c-1} \\
y^{c-1}(x+y) & x^{c}
\end{array}\right] .
\end{aligned}
$$

The polynomials $x^{c}$ and $y^{c-1}(x+y)$ each appear as entries in each matrix. Each matrix $\varphi_{\natural}$ has the correct form to be in $\mathrm{DO}_{\natural}$. It is not difficult to see that ht $I_{2}\left(\varphi_{\natural}\right)=$ 2 for each $\downarrow$. Notice when calculating $I_{2}\left(\varphi\left(\emptyset, \mu_{i}\right)\right)$ that one of the $2 \times 2$ minors is equal to $\pm x^{c-1} y^{c+1}$. Indeed, when $i$ is 5 or 6 , then the minor obtained by deleting row 1 of $\varphi_{\left(\emptyset, \mu_{i}\right)}$ is

$$
\left|\begin{array}{cc}
y^{c} & y x^{c-1} \\
y^{c-1}(x+y) & x^{c}
\end{array}\right|=x^{c-1} y^{c-1}\left|\begin{array}{cc}
y & y \\
x+y & x
\end{array}\right|=-x^{c-1} y^{c+1} .
$$

The comparable minor for $\emptyset=\left(\emptyset, \mu_{4}\right)$ involves rows 1 and 3 . If $\emptyset=\left(\emptyset, \mu_{4}\right)$ then the appropriate entries of $\varphi_{\natural}$ are linearly independent provided $3 \leq c$. If $\downarrow=\left(\emptyset, \mu_{5}\right)$, then the appropriate entries of $\varphi_{\natural}$ are linearly independent provided $4 \leq c$. If $5 \leq c$, then all entries of $\varphi_{\emptyset, \mu_{6}}$ are linearly independent. Apply Lemma 4.19. 
SeCtion 5. The SPACE OF TRUE TRIPLES OF FORMS OF DEGREE $d$ : THE BASE POINT FREE LOCUS, THE BIRATIONAL LOCUS, AND THE GENERIC HILBERT-Burch MATRIX.

In the previous sections we considered one rational curve at a time. At this point our attention turns to the family of all rational plane curves of degree $d$. This family is parameterized by the space $\mathbb{T}_{d}$ of true triples of forms of degree $d$. The space $\mathbb{T}_{d}$ sits naturally in the affine space $\mathbb{A}_{d}$.

Definition 5.1. Let $\boldsymbol{k}$ be a field, $B$ be the polynomial ring $B=\boldsymbol{k}[x, y]$, and $d$ be a positive integer. Define

$$
\mathbb{A}_{d}=B_{d} \times B_{d} \times B_{d} .
$$

Remark 5.2. Each element of $\mathbb{A}_{d}$ is an ordered triple $\boldsymbol{g}=\left(g_{1}, g_{2}, g_{3}\right)$ of homogeneous forms of degree $d$ from the polynomial ring $B$. The space $\mathbb{A}_{d}$ is isomorphic to affine space $\mathbb{A}^{3 d+3}$. If $\boldsymbol{g} \in \mathbb{A}_{d}$, then the corresponding element of $\mathbb{A}^{3 d+3}$ is denoted $\lambda_{\boldsymbol{g}}$; and if $\lambda \in \mathbb{A}^{3 d+3}$, then the corresponding element of $\mathbb{A}_{d}$ is denoted $\boldsymbol{g}_{\lambda}$. The correspondence is given as follows. If $\boldsymbol{g}=\left(g_{1}, g_{2}, g_{3}\right) \in \mathbb{A}_{d}$, with $g_{j}=\sum_{i=0}^{d} \lambda_{i, j} x^{i} y^{d-i}$, then

$$
\lambda_{\boldsymbol{g}}=\left(\lambda_{0,1}, \ldots, \lambda_{d, 1}, \lambda_{0,2}, \ldots, \lambda_{d, 2}, \lambda_{0,3}, \ldots, \lambda_{d, 3}\right) .
$$

Definition 5.3. Fix an element $\boldsymbol{g}=\left(g_{1}, g_{2}, g_{3}\right) \in \mathbb{A}_{d}$. Define $I_{\boldsymbol{g}}$ to be the ideal $\left(g_{1}, g_{2}, g_{3}\right) B$ of $B$ and $\Psi_{\boldsymbol{g}}$ to be the morphism

$$
\Psi_{\boldsymbol{g}}: \mathbb{P}^{1} \backslash V\left(I_{\boldsymbol{g}}\right) \rightarrow \mathbb{P}^{2},
$$

which is given by

$$
\Psi_{\boldsymbol{g}}(q)=\left[g_{1}(q): g_{2}(q): g_{3}(q)\right],
$$

for each point $q$ in $\mathbb{P}^{1}$, where $V\left(I_{\boldsymbol{g}}\right)$ is the zero locus in $\mathbb{P}^{1}$ of the ideal $I_{\boldsymbol{g}}$. Define the curve $\mathcal{C}_{\boldsymbol{g}}$ to be the closure of the image of $\Psi_{\boldsymbol{g}}$, and define $\boldsymbol{d}_{1}(\boldsymbol{g})$ to be the row vector $\left[\begin{array}{lll}g_{1} & g_{2} & g_{3}\end{array}\right]$.

Definition 5.4. Define subsets $\mathrm{BPF}_{d}$, $\operatorname{Bir}_{d}$, and $\mathbb{T}_{d}$ of $\mathbb{A}_{d}$ as follows:

$$
\begin{aligned}
\mathrm{BPF}_{d} & =\left\{\boldsymbol{g} \in \mathbb{A}_{d} \mid \text { the rational map } \Psi_{\boldsymbol{g}} \text { is base point free }\right\} \\
\mathrm{Bir}_{d} & =\left\{\boldsymbol{g} \in \mathbb{A}_{d} \mid \text { the rational map } \Psi_{\boldsymbol{g}} \text { is birational }\right\} \\
\mathbb{T}_{d} & =\mathrm{BPF}_{d} \cap \mathrm{Bir}_{d}=\left\{\boldsymbol{g} \in \mathbb{A}_{d} \mid \begin{array}{l}
\Psi_{\boldsymbol{g}} \text { is a birational morphism with } \\
\text { no base points }
\end{array}\right\} .
\end{aligned}
$$

If $d$ is even, then define subsets $\mathrm{Bal}_{d}, \mathbb{B}_{d}$, and $\mathbb{U B}_{d}$ of $\mathbb{A}_{d}$ as follows:

$$
\begin{aligned}
\mathrm{Bal}_{d} & =\left\{\boldsymbol{g} \in \mathbb{A}_{d} \mid \begin{array}{l}
\text { every entry in a homogeneous Hilbert-Burch } \\
\text { matrix for } \boldsymbol{d}_{1}(\boldsymbol{g}) \text { has degree } d / 2
\end{array}\right. \\
\mathbb{B}_{d} & =\mathrm{Bal}_{d} \cap \mathbb{T}_{d} \\
\mathbb{U B}_{d} & =\mathbb{T}_{d} \backslash \mathbb{B}_{d} .
\end{aligned}
$$


Remark 5.5. We call $\mathbb{T}_{d}$ the space of true triples of forms of degree $d, \mathbb{B}_{d}$ the space of balanced true triples of forms of degree $d$, and $\mathbb{U B}_{d}$ the space of unbalanced true triples of forms of degree $d$. The true triples of $\mathbb{B}_{d}$ are called "balanced" because the corresponding Hilbert-Burch matrices are balanced in the sense that the column degrees $d_{1}$ and $d_{2}$ (in the language of Data 1.1) are equal; an unbalanced Hilbert-Burch matrix has $d_{1}<d_{2}$. Keep in mind that if $\boldsymbol{g}$ is in $\mathbb{A}_{d}$, then

$$
\boldsymbol{g} \in \mathbb{B}_{d} \Longleftrightarrow \begin{aligned}
& \text { the morphsim } \Psi_{\boldsymbol{g}} \text { is Birational, Base point free and the } \\
& \text { Hilbert-Burch matrix for } \boldsymbol{d}_{1}(\boldsymbol{g}) \text { is Balanced. }
\end{aligned}
$$

In Theorems 5.20 and 5.24 we prove that $\mathbb{T}_{d}$ and $\mathbb{B}_{d}$ are open subsets of $\mathbb{A}_{d}$.

In practice we are only interested in the open subset $\mathbb{T}_{d}$ of $\mathbb{A}_{d}$. Every element $\boldsymbol{g}$ of $\mathbb{A}_{d}$ which is not in $\mathbb{T}_{d}$ corresponds to an unsuitable parameterization of the curve $\mathcal{C}_{\boldsymbol{g}}$. Some of these unsuitable parameterizations have base points; others are not birational. One can remove base points by factoring out and removing the greatest common factor of the parameterizing forms. Also, if the parameterization is not birational, then one can reparameterize to find a birational parameterization; see, for example, Theorem 0.10 or [31, Section 6.1]. Notice that if $\boldsymbol{g}$ is in $\mathbb{A}_{d}$, then the data $\Psi_{\boldsymbol{g}}, I_{\boldsymbol{g}}$, and $\mathcal{C}_{\boldsymbol{g}}$ are uniquely determined by $\boldsymbol{g}$; furthermore,

$$
\begin{aligned}
& \text { the data }\left(\Psi_{\boldsymbol{g}}, I_{\boldsymbol{g}}, \mathcal{C}_{\boldsymbol{g}}\right) \text { satisfy the } \\
& \text { conditions and hypotheses of Data } 1.1 \Longleftrightarrow \boldsymbol{g} \in \mathbb{T}_{d} \text {. }
\end{aligned}
$$

Let $\mathbb{P T}_{d}$ represent the space of true parameterizations of plane curves of degree $d$; that is,

$$
\mathbb{P T}_{d}=\left\{\begin{array}{l|l}
\text { morphisms } \Psi: \mathbb{P}^{1} \rightarrow \mathbb{P}^{2} & \begin{array}{l}
\Psi \text { is birational, base point free, and has } \\
\text { degree } d
\end{array}
\end{array}\right\}
$$

We have established the following statement, where $\boldsymbol{k}^{*}$ means $\boldsymbol{k} \backslash\{0\}$ and $u\left(g_{1}, g_{2}, g_{3}\right)$ means $\left(u g_{1}, u g_{2}, u g_{3}\right)$.

Observation 5.7. The function $\mathbb{T}_{d} \rightarrow \mathbb{P T}_{d}$, which is given by $\boldsymbol{g} \mapsto \Psi_{\boldsymbol{g}}$, is surjective and the fiber over $\Psi_{\boldsymbol{g}}$ is $\left\{u \boldsymbol{g} \mid u \in \boldsymbol{k}^{*}\right\}$.

If $d=2 c$ is an even integer, then the open subset $\mathbb{B}_{d}$ of $\mathbb{T}_{d}$ is of particular interest to us because one of the main topics of study in this paper is singularities of multiplicity $c$ on, or infinitely near, curves of degree $d$. We proved in Corollary 2.5 that if such a singularity exists for the curve $\mathcal{C}_{\boldsymbol{g}}$, with $\boldsymbol{g} \in \mathbb{T}_{d}$, then $\boldsymbol{g}$ must be in $\mathbb{B}_{d}$.

In the second half of this section we return to the idea of (5.6). In order to have all of the data of 1.1 one also must identify a Hilbert-Burch matrix for $\boldsymbol{d}_{1}(\boldsymbol{g})$. It 
is not possible to define a unique Hilbert-Burch matrix for $\boldsymbol{d}_{1}(\boldsymbol{g})$ as a function of $\boldsymbol{g}$ over all of $\mathbb{T}_{d}$; however, if we restrict our attention to $\mathbb{B}_{d}$, then this is almost possible. In Corollary 5.36 we identify a complex $\mathbb{F}$ of free $(\boldsymbol{k}[\boldsymbol{z}])[x, y]$-modules, where $\boldsymbol{z}$ is the $1 \times(3 d+3)$ matrix

$$
\boldsymbol{z}=\left[z_{0,1}, \ldots, z_{d, 1}, z_{0,2}, \ldots, z_{d, 2}, z_{0,3}, \ldots, z_{d, 3}\right]
$$

of indeterminates and we prove that for $\lambda \in \mathbb{A}^{3 d+3}$,

$$
\mathbb{F} \otimes_{\boldsymbol{k}[\boldsymbol{z}]} \frac{\boldsymbol{k}[\boldsymbol{z}]}{(\boldsymbol{z}-\lambda)} \text { is a resolution of } I_{\boldsymbol{g}_{\lambda}} \Longleftrightarrow \boldsymbol{g}_{\lambda} \in \mathrm{Bal}_{d}
$$

The complex $\mathbb{F}$ would furnish a generic Hilbert-Burch matrix for $\boldsymbol{d}_{1}(\boldsymbol{g})$ for $\boldsymbol{g} \in \mathrm{Bal}_{d}$ except, unfortunately, the rank of $\mathbb{F}_{2}$ is three instead of two. There are three ways to interpret what we do get. First of all, in Theorem 5.30, we identify an open cover $\cup_{i=1}^{3} \mathrm{Bal}_{d}^{(i)}$ of $\mathrm{Bal}_{d}$ and matrices $\boldsymbol{d}_{2}^{(i)}$, for $1 \leq i \leq 3$, in $(\boldsymbol{k}[\boldsymbol{z}])[x, y]$ such that if $\boldsymbol{g} \in \mathrm{Bal}_{d}^{(i)}$, then $\boldsymbol{d}_{2}^{(i)} \otimes_{\boldsymbol{k}[\boldsymbol{z}]} \frac{\boldsymbol{k}[\boldsymbol{z}]}{\left(\boldsymbol{z}-\lambda_{\boldsymbol{g}}\right)}$ is a Hilbert-Burch matrix for $\boldsymbol{d}_{1}(\boldsymbol{g})$. Secondly, in Corollary 5.46 we identify a universal projective resolution $\mathbb{U P} \mathbb{R}_{\mathbb{Z}}$ for all resolutions with graded Betti numbers

$$
0 \rightarrow \boldsymbol{k}[x, y](-3 c)^{2} \rightarrow \boldsymbol{k}[x, y](-2 c)^{3} \rightarrow \boldsymbol{k}[x, y]
$$

Do notice that the module in position 2 of $\mathbb{U P R} \mathbb{R}_{\mathbb{Z}}$ is a projective module which is not necessarily free. Finally, in Section 4 we studied the morphism $\Phi: \mathbb{H}_{d} \rightarrow \mathbb{A}_{d}$ which sends a $3 \times 2$ matrix with entries from $\boldsymbol{k}[x, y]_{c}$ to a triple $\boldsymbol{g}$ of $\mathbb{A}_{d}$. One may restrict $\Phi$ to become

$$
\Phi \mid: \Phi^{-1}\left(\mathrm{Bal}_{d}\right) \rightarrow \mathrm{Bal}_{d}
$$

Corollary 5.45 gives a local section of the morphism (5.10).

Before we get to work we make one small observation about how the subsets of Definition 5.4 fit together.

Observation 5.11. If $d=2 c$ is an even integer, then $\mathrm{Bal}_{d} \subseteq \mathrm{BPF}_{d}$; however, in general, $\mathrm{Bal}_{d} \not \subset \mathrm{Bir}_{d}$.

Proof. The triple $\boldsymbol{g}=\left(x^{d}, x^{c} y^{c}, y^{d}\right)$ of $\mathbb{A}_{d}$ is in $\mathrm{Bal}_{d}$, but does not correspond to a birational parameterization of the curve $T_{2}^{2}=T_{1} T_{3}$ unless $d=2$. On the other hand, if $\boldsymbol{g}=\left(g_{1}, g_{2}, g_{3}\right)$ is any non-zero element of $\mathbb{A}_{d}$, then the Hilbert-Burch Theorem shows that the graded Betti numbers of $B / I$ are

$$
0 \rightarrow B\left(-d-d_{2}\right) \oplus B\left(-d-d_{1}\right) \rightarrow B(-d)^{3} \rightarrow B
$$


for some non-negative integers $d_{1}$ and $d_{2}$ with $d_{1}+d_{2}+\operatorname{deg} \operatorname{gcd}\left(g_{1}, g_{2}, g_{3}\right)=d$. If $\boldsymbol{g} \in \mathrm{Bal}_{d}$, then $d_{1}+d_{2}=c+c=d$, the gcd of $\left(g_{1}, g_{2}, g_{3}\right)$ is a unit, the height of $I_{\boldsymbol{g}}$ is 2 , and $\Psi_{\boldsymbol{g}}$ has no base points.

Theorem 5.13 is our main tool for proving that subsets of $\mathbb{A}_{d}$ are closed. We apply Theorem 5.13 in Theorem 5.20 when we prove that $\mathbb{T}_{d}$ is an open subset of $\mathbb{A}_{d}$; we also apply Theorem 5.13 in throughout Section 6 when we decompose $\mathbb{B}_{d}$ into locally closed strata. The first assertion in the statement below is well-known. The proof we offer for Theorem 5.13 is based on the Generic Freeness Lemma and is inspired by the proof of assertion (1) which is given by Eisenbud [11, Thm. 14.8]. We prove both assertions simultaneously. If $p \in \operatorname{Spec} R$, then

$$
\boldsymbol{k}(p) \text { denotes the residue class field } R_{p} / p R_{p} \text { of the local ring } R_{p}
$$

Theorem 5.13. Let $S$ be a standard graded Noetherian algebra over $S_{0}=R$. For non-negative integers $d$ and $e$, define

$$
\left.\begin{array}{rl}
X(\geq d) & =\quad X(S ; \geq d)=\left\{p \in \operatorname{Spec} R \mid \operatorname{dim} S \otimes_{R} \boldsymbol{k}(p) \geq d\right\} \\
X(=d) & =\quad X(S ;=d)=\left\{p \in \operatorname{Spec} R \mid \operatorname{dim} S \otimes_{R} \boldsymbol{k}(p)=d\right\} \\
X(=d, \geq e) & =X(S ;=d, \geq e)=\left\{p \in \operatorname{Spec} R \mid \begin{array}{l}
\operatorname{dim} S \otimes_{R} \boldsymbol{k}(p)=d \\
e\left(S \otimes_{R} \boldsymbol{k}(p)\right) \geq e
\end{array}\right.
\end{array}\right\} .
$$

Then

(1) $X(S ; \geq d)$ is a closed subset of $\operatorname{Spec} R$, and

(2) $X(S ;=d, \geq e)$ is a closed subset of $X(S ;=d)$.

Note. Once one has identified the ring $S$, then the degree zero component $S_{0}=R$ is automatically determined. For this reason we have denoted with $X(S ; \geq d)$ the subset $X(\geq d)$ of $\operatorname{Spec} R$.

Proof. The proof proceeds by induction on the dimension of $R$. If $\operatorname{dim} R=0$, then $\operatorname{Spec} R$ is finite and every subset of $\operatorname{Spec} R$ is closed. Henceforth, we assume that $\operatorname{dim} R$ is positive.

We next reduce to the case where $R$ is a domain. Let $\left\{p_{1}, \ldots, p_{s}\right\}$ be the set of minimal prime ideals of $R$. Observe that

$$
\begin{aligned}
X(\geq d) & =\bigcup_{i=1}^{s}\left(X(\geq d) \cap V\left(p_{i}\right)\right)=\bigcup_{i=1}^{s}\left(X\left(S \otimes_{R} R / p_{i} ; \geq d\right),\right. \text { and } \\
X(=d, \geq e) & =\bigcup_{i=1}^{s}\left(X(=d, \geq e) \cap V\left(p_{i}\right)\right)=\bigcup_{i=1}^{s}\left(X\left(S \otimes_{R} R / p_{i} ;=d, \geq e\right)\right) .
\end{aligned}
$$


Once we prove the result for each $R / p_{i} \subseteq S \otimes_{R} R / p_{i}$, then we have also established the result for $R \subseteq S$. Henceforth, we also assume that $R$ is a domain.

Now we apply the Generic Freeness Lemma. The ring $R$ is a domain and $S$ is a finitely generated $R$-algebra; so there exists a non-zero element $a$ of $R$ such that $S_{a}=S \otimes_{R} R_{a}$ is a free $R_{a}$-module. We notice that $S_{a}$ is still a standard graded $R_{a}$-algebra with degree zero component equal to $R_{a}$; furthermore, each component $\left[S \otimes_{R} R_{a}\right]_{i}$ is finitely generated free $R_{a}$-module.

Let $K=\operatorname{Quot}(R)$. Take $p$ in the open subset $D(a)=\operatorname{Spec} R \backslash V(a)$, and take $q$ in Spec $R$. (In this context, $V(a)$ is the closed subset $\{p \in \operatorname{Spec} R \mid a \in p\}$ of Spec $R$.) We make the following observations about the Hilbert functions $\mathrm{H}_{S \otimes_{R} \boldsymbol{k}(p)}(i)$ and $\mathrm{H}_{S \otimes_{R} \boldsymbol{k}(q)}(i):$

$$
\begin{aligned}
\mathrm{H}_{S \otimes_{R} \boldsymbol{k}(p)}(i) & =\lambda_{R_{p}}\left[S \otimes_{R} \boldsymbol{k}(p)\right]_{i}=\mu_{R_{p}}\left[S \otimes_{R} R_{p}\right]_{i}=\operatorname{dim}_{K}\left[S \otimes_{R} K\right]_{i} \\
& =\mu_{K}\left[S \otimes_{R} K\right]_{i} \leq \mu_{R_{q}}\left[S \otimes_{R} R_{q}\right]_{i}=\lambda_{R_{q}}[S \otimes \boldsymbol{k}(q)]_{i}=\mathrm{H}_{S \otimes_{R} \boldsymbol{k}(q)}(i) .
\end{aligned}
$$

The first and last equalities are the definition of Hilbert function. The second equality and second from last equalities follow from Nakayama's Lemma. The third equality is due to the fact that $\left[S \otimes_{R} R_{p}\right]_{i}$ is a free $R_{p}$-module. The fourth equality and the inequality are obvious. We have shown that

$$
\begin{aligned}
& \mathrm{H}_{S \otimes_{R} \boldsymbol{k}(p)}(i)=\mathrm{H}_{S \otimes_{R} K}(i), \text { for all } p \in D(a), \text { and } \\
& \mathrm{H}_{S \otimes_{R} \boldsymbol{k}(p)}(i) \leq \mathrm{H}_{S \otimes_{R} \boldsymbol{k}(q)}(i) \text { for all } p \in D(a) \text { and } q \in \operatorname{Spec} R
\end{aligned}
$$

Let $d_{0}=\operatorname{dim} S \otimes_{R} K$, and $e_{0}=e\left(S \otimes_{R} K\right)$. The Krull dimension of a standard graded algebra over a field may be read from its Hilbert function; hence, (5.14) shows that

$$
d_{0}=\operatorname{dim} S \otimes_{R} \boldsymbol{k}(p) \leq \operatorname{dim} S \otimes_{R} \boldsymbol{k}(q) \text { for all } p \in D(a) \text { and } q \in \operatorname{Spec} R \text {. }
$$

Furthermore, once the dimension of a standard graded algebra is determined, then its multiplicity may also be read from its Hilbert function. It follows that

$$
e_{0}=e\left(S \otimes_{R} \boldsymbol{k}(p)\right) \leq e\left(S \otimes_{R} \boldsymbol{k}(q)\right)
$$

for all $p \in D(a)$ and $q \in \operatorname{Spec} R$ with $\operatorname{dim} S \otimes_{R} \boldsymbol{k}(q)=d_{0}$.

The Krull dimension of $R /(a)$ is less than the Krull dimension of $R$ and so

$$
\begin{aligned}
& V(a) \cap X(S ; \geq d)=X\left(S \otimes_{R} R /(a) ; \geq d\right) \quad \text { and } \\
& V(a) \cap X(S ;=d, \geq e)=X\left(S \otimes_{R} R /(a) ;=d, \geq e\right)
\end{aligned}
$$


are closed subsets of $\operatorname{Spec} R /(a)$ and $X\left(S \otimes_{R} R /(a) ;=d\right)$, respectively, by the induction hypothesis.

We first consider the case $d>d_{0}$. In this case, $X(S ; \geq d)=X(S ; \geq d) \cap V(a)$ is a closed subset of $\operatorname{Spec} R /(a)$; hence, a closed subset of Spec $R$. Also in this case, $X(S ;=d, \geq e)=X(S ;=d, \geq e) \cap V(a)$ is a closed subset of

$$
X\left(S \otimes_{R} R /(a) ;=d\right)=X(S ;=d) .
$$

Finally, we consider the case $d=d_{0}$. In this case, $X\left(S ; \geq d_{0}\right)=\operatorname{Spec} R$. If $e>e_{0}$, then $X\left(S ;=d_{0}, \geq e\right)=X\left(S ;=d_{0}, \geq e\right) \cap V(a)$ is a closed subset of

$$
X\left(S \otimes_{R} R /(a) ;=d_{0}\right)=V(a) \cap\left(X\left(S ;=d_{0}\right) ;\right.
$$

and therefore $X\left(S ;=d_{0}, \geq e\right)$ is a closed subset of $X\left(S ;=d_{0}\right)$. If $e=e_{0}$, then $X\left(S ;=d_{0}, \geq e_{0}\right)=X\left(S ;=d_{0}\right)$.

Conventions 5.15. Let $\boldsymbol{k}$ be a field.

(1) We denote the coordinate ring of $\mathbb{A}^{3 d+3}$ by $\boldsymbol{R}=\boldsymbol{k}[\boldsymbol{z}]$, where $\boldsymbol{z}$ is a $1 \times(3 d+3)$ matrix of indeterminates given in (5.8). We continue to write $B$ for the standard graded polynomial ring $\boldsymbol{k}[x, y]$. Let $\boldsymbol{S}$ be the bi-graded polynomial $\operatorname{ring} \boldsymbol{S}=\boldsymbol{k}[x, y, \boldsymbol{z}]$, where $x$ and $y$ each have bi-degree $(1,0)$, and $z_{i, j}$ has bi-degree $(0,1)$.

(2) For each $\lambda \in \mathbb{A}^{3 d+3}$, let $\boldsymbol{R}_{\lambda}$ denote the $\operatorname{ring} \boldsymbol{R} / \mathfrak{m}_{\lambda}=\boldsymbol{k}\left(\mathfrak{m}_{\lambda}\right)=\boldsymbol{k}$ where $\mathfrak{m}_{\lambda}$ is the maximal ideal $\left(\left\{z_{i, j}-\lambda_{i, j}\right\}\right)$ of $\boldsymbol{R}$. (Recall the notation $\boldsymbol{k}(p)$ for residue class field from (5.12).) If $S$ is an $\boldsymbol{R}$-algebra, then let $S_{\lambda}$ denote $S \otimes_{\boldsymbol{R}} \boldsymbol{R}_{\lambda}$ and if $G \in S$, then let $\left.G\right|_{\lambda}$ (read as " $G$ evaluated at $\lambda$ ") be the image of $G$ in $S_{\lambda}$.

(3) For each index $j$, with $1 \leq j \leq 3$, let $G_{j}$ be the polynomial

$$
G_{j}=\sum_{i=0}^{d} z_{i, j} x^{i} y^{d-i}
$$

in $\boldsymbol{S}$. If $\boldsymbol{g}=\left(g_{1}, g_{2}, g_{3}\right) \in \mathbb{A}_{d}$, then, according to Convention (2) and Remark 5.2, $\left.G_{j}\right|_{\lambda_{\boldsymbol{g}}}$ is equal to $g_{j}$ in $\boldsymbol{S}_{\lambda_{\boldsymbol{g}}}=B$.

(4) We often apply Theorem 5.13 to the affine space $\mathbb{A}_{d}$ by way of the identification of $\mathbb{A}_{d}$ and $\mathbb{A}^{3 d+3}$ which is given in Remark 5.2. Of course, the Zariski topology on $\mathbb{A}^{3 d+3}$ is the same as the subspace topology that $\mathbb{A}^{3 d+3}$ inherits as subset of $\operatorname{Max} \operatorname{Spec} \boldsymbol{R} \subseteq \operatorname{Spec} \boldsymbol{R}$. In this language, Theorem 5.13 shows that if $S$ is a standard graded Noetherian algebra over $S_{0}=\boldsymbol{R}$ and $a$ and $b$ are non-negative integers then

$$
X(S ; \geq a) \cap \mathbb{A}_{d}=\left\{\boldsymbol{g} \in \mathbb{A}_{d} \mid \operatorname{dim} S_{\lambda_{\boldsymbol{g}}} \geq a\right\}
$$

is a closed subset of $\mathbb{A}_{d}$ and

$$
X(S ;=a, \geq b) \cap \mathbb{A}_{d}=\left\{\boldsymbol{g} \in \mathbb{A}_{d} \mid \operatorname{dim} S_{\lambda_{\boldsymbol{g}}}=a \quad \text { and } \quad e\left(S_{\lambda_{\boldsymbol{g}}}\right) \geq b\right\}
$$

is a closed subset of $X(S ;=a) \cap \mathbb{A}_{d}=\left\{\boldsymbol{g} \in \mathbb{A}_{d} \mid \operatorname{dim} S_{\lambda_{\boldsymbol{g}}}=a\right\}$. 
Proposition 5.17. The subset $\mathrm{BPF}_{d}$ of $\mathbb{A}_{d}$ is open.

Proof. Let $\boldsymbol{R}=\boldsymbol{k}[\boldsymbol{z}]$ be the coordinate ring of $\mathbb{A}^{3 d+3}$, as described in item (1) of Conventions 5.15, and $S$ be the $\boldsymbol{R}$-algebra $\boldsymbol{R}[x, y] /\left(G_{1}, G_{2}, G_{3}\right)$, where the polynomials $G_{j}$ are described in (5.16). Fix an element $\boldsymbol{g} \in \mathbb{A}_{d}$. Observe that $S_{\lambda_{\boldsymbol{g}}}$ is equal to $\boldsymbol{k}[x, y] / I_{\boldsymbol{g}}$; furthermore,

$$
\begin{aligned}
\boldsymbol{g} \in \mathbb{A}_{d} \backslash \mathrm{BPF}_{d} & \Longleftrightarrow \Psi_{\boldsymbol{g}} \text { has base points } \Longleftrightarrow \operatorname{ht} I_{\boldsymbol{g}} \leq 1 \\
& \Longleftrightarrow \operatorname{dim} \boldsymbol{k}[x, y] / I_{\boldsymbol{g}} \geq 1 \Longleftrightarrow \operatorname{dim} S_{\lambda_{\boldsymbol{g}}} \geq 1 .
\end{aligned}
$$

Thus,

$$
\mathbb{A}_{d} \backslash \mathrm{BPF}_{d}=\left\{\boldsymbol{g} \in \mathbb{A}_{d} \mid \operatorname{dim} S_{\lambda_{\boldsymbol{g}}} \geq 1\right\}=X(S ; \geq 1) \cap \mathbb{A}_{d},
$$

which is a closed subset of $\mathbb{A}_{d}$ by Theorem 5.13 by way of item (4) of Conventions 5.15 .

Proposition 5.18. The subset $\operatorname{Bir}_{d}$ of $\mathbb{A}_{d}$ is open.

Proof. Let $\boldsymbol{R}=\boldsymbol{k}[\boldsymbol{z}]$ be the coordinate ring of $\mathbb{A}^{3 d+3}$ as described in Conventions 5.15. Form polynomials $G_{1}, G_{2}, G_{3}$ in $\boldsymbol{R}[x, y]$ also as described in Conventions 5.15. Consider the set

$$
\mathcal{G}=\left\{G_{1}^{i} G_{2}^{j} G_{3}^{k} \mid i+j+k=d-1\right\}
$$

of $\left(\begin{array}{c}d+1 \\ 2\end{array}\right)$ polynomials in $\boldsymbol{R}[x, y]$. Each element of $\mathcal{G}$ is a polynomial of degree $d(d-1)$ in the variables $x, y$ with coefficients coming from the ring $\boldsymbol{R}$. Let $Z$ be the $(d(d-1)+1) \times\left(\begin{array}{c}d+1 \\ 2\end{array}\right)$ matrix which expresses the elements of $\mathcal{G}$ in terms of the usual monomial basis for $\boldsymbol{k}[x, y]_{d(d-1)}$, and let $\mathfrak{a}$ be the ideal in $\boldsymbol{R}$ generated by the maximal minors of $Z$; that is $\mathfrak{a}=I_{\left(\begin{array}{c}d+1 \\ 2\end{array}\right)}(Z)$.

Take $\boldsymbol{g}=\left(g_{1}, g_{2}, g_{3}\right) \in \mathbb{A}_{d}$. Item (3) of Conventions 5.15 shows that $\left.G_{1}^{i} G_{2}^{j} G_{3}^{k}\right|_{\lambda_{\boldsymbol{g}}}$ is equal to $g_{1}^{i} g_{2}^{j} g_{3}^{k}$. Observe that the polynomials

$$
g_{1}^{i} g_{2}^{j} g_{3}^{k}, \quad \text { with } i+j+k=d-1
$$

are linearly independent in $\boldsymbol{k}\left[g_{1}, g_{2}, g_{3}\right] \subseteq \boldsymbol{k}[x, y]$ if and only if $\lambda_{\boldsymbol{g}}$ is not in $V(\mathfrak{a})$.

The ring $\boldsymbol{k}\left[g_{1}, g_{2}, g_{3}\right]$ is the coordinate ring of the curve $\mathcal{C}_{\boldsymbol{g}}$. Furthermore, the ring homomorphism $\boldsymbol{k}\left[T_{1}, T_{2}, T_{3}\right] \rightarrow \boldsymbol{k}\left[g_{1}, g_{2}, g_{3}\right]$, which sends $T_{i}$ to $g_{i}$, induces an isomorphism

$$
\frac{\boldsymbol{k}\left[T_{1}, T_{2}, T_{3}\right]}{\left(f_{\boldsymbol{g}}\right)} \cong \boldsymbol{k}\left[g_{1}, g_{2}, g_{3}\right]
$$


where $f_{\boldsymbol{g}}$ is the defining equation of the curve $\mathcal{C}_{\boldsymbol{g}}$. The degree of $f_{\boldsymbol{g}}$ is equal to the multiplicity $e$ of $\boldsymbol{k}\left[g_{1}, g_{2}, g_{3}\right]$ and $\Psi_{\boldsymbol{g}}$ is a birational morphism if and only if $e=d$; see, for example, Theorem 0.10. Thus,

$$
\begin{aligned}
\Psi_{\boldsymbol{g}} \text { is birational } & \Longleftrightarrow \operatorname{deg} f_{\boldsymbol{g}}=d \\
& \Longleftrightarrow \operatorname{dim}_{\boldsymbol{k}}\left(\boldsymbol{k}\left[T_{1}, T_{2}, T_{3}\right] /\left(f_{\boldsymbol{g}}\right)\right)_{d-1} \geq \operatorname{dim}_{\boldsymbol{k}} \boldsymbol{k}\left[T_{1}, T_{2}, T_{3}\right]_{d-1} \\
& \Longleftrightarrow \operatorname{dim}_{\boldsymbol{k}} \boldsymbol{k}\left[g_{1}, g_{2}, g_{3}\right]_{d-1} \geq\left(\begin{array}{c}
d+1 \\
2
\end{array}\right) \\
& \Longleftrightarrow \text { the elements of }\left.\mathcal{G}\right|_{\lambda_{\boldsymbol{g}}} \text { are linearly independent } \\
& \text { in } \boldsymbol{k}\left[g_{1}, g_{2}, g_{3}\right] \subseteq \boldsymbol{k}[x, y] \\
& \Longleftrightarrow \lambda_{\boldsymbol{g}} \notin V(\mathfrak{a}) . \quad \square
\end{aligned}
$$

Theorem 5.20. The subset $\mathbb{T}_{d}$ of $\mathbb{A}_{d}$ is open.

Proof. The set $\mathbb{T}_{d}$ is equal to $\mathrm{BPF}_{d} \cap \mathrm{Bir}_{d}$. Apply Propositions 5.17 and 5.18.

Most of our work takes place over a field $\boldsymbol{k}$. However, Theorem 5.41, Corollary 5.46, and Corollary 5.48 are about 3 generic forms of the same even degree in the polynomial ring $\mathbb{Z}[x, y]$. In order to facilitate the transition from working over a field to working over the integers, we set up our data over $\mathbb{Z}$ right from the beginning. Every polynomial with coefficients in $\mathbb{Z}$ automatically represents a unique polynomial with coefficients in $\boldsymbol{k}$.

Definition 5.21. Let $d=2 c$ be a positive even integer and $\boldsymbol{S}_{\mathbb{Z}}$ be the bi-graded polynomial ring $\boldsymbol{S}_{\mathbb{Z}}=\mathbb{Z}[x, y, \boldsymbol{z}]$, where $\boldsymbol{z}$ is the $1 \times(3 d+3)$ matrix of indeterminates given in (5.8), $\operatorname{deg} x=\operatorname{deg} y=(1,0)$, and $\operatorname{deg} z_{i, j}=(0,1)$. For each index $j$, with $1 \leq j \leq 3$, let $G_{j}$ be the bi-homogeneous polynomial of (5.16) in $\boldsymbol{S}_{\mathbb{Z}}$ of degree $(d, 1)$. Let $\Delta_{\mathbb{Z}}$ be the ring $\Delta_{\mathbb{Z}}=S_{\mathbb{Z}} /\left(G_{1}, G_{2}, G_{3}\right)$. Let $\boldsymbol{R}_{\mathbb{Z}}$ be the standard graded polynomial ring $\boldsymbol{R}_{\mathbb{Z}}=\mathbb{Z}[\boldsymbol{z}]$.

(1) For each positive integer $i$, let $A_{\mathbb{Z}}^{(i)}$ be the $(d+i+1) \times 3(i+1)$ matrix

$$
A_{\mathbb{Z}}^{(i)}=\left[\begin{array}{cccccccccccc}
z_{0,1} & 0 & \cdots & 0 & z_{0,2} & 0 & \cdots & 0 & z_{0,3} & 0 & \cdots & 0 \\
z_{1,1} & z_{0,1} & \cdots & 0 & z_{1,2} & z_{0,2} & \cdots & 0 & z_{1,3} & z_{0,3} & \cdots & 0 \\
z_{2,1} & z_{1,1} & \cdots & 0 & z_{2,2} & z_{1,2} & \cdots & 0 & z_{2,3} & z_{1,3} & \cdots & 0 \\
\vdots & \vdots & \cdots & \vdots & \vdots & \vdots & \cdots & \vdots & \vdots & \vdots & \cdots & \vdots \\
z_{d-1,1} & z_{d-2,1} & \cdots & \vdots & z_{d-1,2} & z_{d-2,2} & \cdots & \vdots & z_{d-1,3} & z_{d-2,3} & \cdots & \vdots \\
z_{d, 1} & z_{d-1,1} & \cdots & \vdots & z_{d, 2} & z_{d-1,2} & \cdots & \vdots & z_{d, 3} & z_{d-1,3} & \cdots & \vdots \\
0 & z_{d, 1} & \cdots & \vdots & 0 & z_{d, 2} & \cdots & \vdots & 0 & z_{d, 3} & \cdots & \vdots \\
\vdots & \vdots & \cdots & \vdots & \vdots & \vdots & \cdots & \vdots & \vdots & \vdots & \cdots & \vdots \\
0 & 0 & \cdots & z_{d, 1} & 0 & 0 & \cdots & z_{d, 2} & 0 & 0 & \cdots & z_{d, 3}
\end{array}\right] .
$$




$\left[\begin{array}{c}0 \\ A_{\mathbb{Z}}(1,2) \\ -A_{\mathbb{Z}}(1,3) \\ \vdots \\ \frac{(-1)^{c+1} A_{\mathbb{Z}}(1, c+1)}{(-1)^{c} A_{\mathbb{Z}}(1, c+2)} \\ (-1)^{c+1} A_{\mathbb{Z}}(1, c+3) \\ \vdots \\ \hline A_{\mathbb{Z}}(1,2 c+2) \\ -A_{\mathbb{Z}}(1,2 c+3) \\ A_{\mathbb{Z}}(1,2 c+4) \\ \vdots \\ (-1)^{c+1} A_{\mathbb{Z}}(1,3 c+3)\end{array}\right]\left[\begin{array}{c}A_{\mathbb{Z}}(1, c+2) \\ -A_{\mathbb{Z}}(2, c+2) \\ A_{\mathbb{Z}}(3, c+2) \\ \vdots \\ (-1)^{c} A_{\mathbb{Z}}(c+1, c+2) \\ 0 \\ c+1 A_{\mathbb{Z}}(c+2, c+3) \\ \vdots \\ \frac{\left(A_{\mathbb{Z}}(c+2,2 c+2)\right.}{-A_{\mathbb{Z}}(c+2,2 c+3)} \\ A_{\mathbb{Z}}(c+2,2 c+4) \\ \vdots \\ (-1)^{c+1} A_{\mathbb{Z}}(c+2,3 c+3)\end{array}\right]\left[\begin{array}{c}A_{\mathbb{Z}}(1,2 c+3) \\ -A_{\mathbb{Z}}(2,2 c+3) \\ A_{\mathbb{Z}}(3,2 c+3) \\ \vdots \\ (-1)^{c+1} A_{\mathbb{Z}}(c+2,2 c+3) \\ (-1)^{c} A_{\mathbb{Z}}(c+3,2 c+3) \\ \vdots \\ -A_{\mathbb{Z}}(2 c+2,2 c+3) \\ 0 \\ A_{\mathbb{Z}}(2 c+3,2 c+4) \\ \vdots \\ (-1)^{c+1} A_{\mathbb{Z}}(2 c+3,3 c+3)\end{array}\right]$

Table 1: The relations $\boldsymbol{b}_{\mathbb{Z}}^{(1)}, \boldsymbol{b}_{\mathbb{Z}}^{(c+2)}$, and $\boldsymbol{b}_{\mathbb{Z}}^{(2 c+3)}$, on $A_{\mathbb{Z}}^{(c)}$ from (3) of Definition 5.21.

Each polynomial $G_{j}$ contributes exactly $i+1$ columns to $A_{\mathbb{Z}}^{(i)}$.

(2) Let $w_{\mathbb{Z}}$ be the determinant of the $3 c \times 3 c$ matrix $A_{\mathbb{Z}}^{(c-1)}$; so, $w_{\mathbb{Z}}$ is a bihomogeneous element of $\boldsymbol{S}_{\mathbb{Z}}$ of degree $(0,3 c)$.

(3) Let $A_{\mathbb{Z}}$ be the $(3 c+1) \times(3 c+3)$ matrix $A_{\mathbb{Z}}^{(c)}$ of $(5.22)$. One may obtain $3 c+3$ "Eagon-Northcott" relations on $A_{\mathbb{Z}}$, by crossing one column of $A_{\mathbb{Z}}$ at a time and computing the signed maximal minors of the resulting $(3 c+1) \times(3 c+2)$ matrix. In particular, when one crosses out columns $1, c+2$, or $2 c+3$ of $A_{\mathbb{Z}}$, one obtains the relations $\boldsymbol{b}_{\mathbb{Z}}^{(1)}, \boldsymbol{b}_{\mathbb{Z}}^{(c+2)}$, and $\boldsymbol{b}_{\mathbb{Z}}^{(2 c+3)}$, on $A_{\mathbb{Z}}$, which are given in Table 1 , where $A_{\mathbb{Z}}(i, j)$ is the determinant of the submatrix of $A_{\mathbb{Z}}$ which is obtained by deleting columns $i$ and $j$. Each $A_{\mathbb{Z}}(i, j)$ is a bi-homogeneous element of $\boldsymbol{S}_{\mathbb{Z}}$ of degree $(0,3 c+1)$.

(4) For each positive integer $i$, recall the $1 \times(i+1)$ matrix $\rho_{\mathbb{Z}}^{(i)}=\left[y^{i}, x y^{i-1}, \ldots, x^{i}\right]$ from $(3.1)$ and let $N_{\mathbb{Z}}^{(i)}$ be the $3 \times 3(i+1)$ matrix

$$
N_{\mathbb{Z}}^{(i)}=\left[\begin{array}{ccc}
\rho_{\mathbb{Z}}^{(i)} & 0 & 0 \\
0 & \rho_{\mathbb{Z}}^{(i)} & 0 \\
0 & 0 & \rho_{\mathbb{Z}}^{(i)}
\end{array}\right] .
$$

Define $\boldsymbol{q}_{1, \mathbb{Z}}=N_{\mathbb{Z}}^{(c)} \boldsymbol{b}_{\mathbb{Z}}^{(1)}, \boldsymbol{q}_{2, \mathbb{Z}}=N_{\mathbb{Z}}^{(c)} \boldsymbol{b}_{\mathbb{Z}}^{(c+2)}$, and $\boldsymbol{q}_{3, \mathbb{Z}}=N_{\mathbb{Z}}^{(c)} \boldsymbol{b}_{\mathbb{Z}}^{(2 c+3)}$. Each $\boldsymbol{q}_{j, \mathbb{Z}}$ is a column vector with three entries and each entry of each $\boldsymbol{q}_{j, \mathbb{Z}}$ is a bi-homogeneous element of $\boldsymbol{S}_{\mathbb{Z}}$ of degree $(c, 3 c+1)$. 
(5) Define the matrix $\boldsymbol{d}_{2, \mathbb{Z}}$ to be $\boldsymbol{d}_{2, \mathbb{Z}}=\left[\begin{array}{lll}\boldsymbol{q}_{1, \mathbb{Z}} & \boldsymbol{q}_{2, \mathbb{Z}} & \boldsymbol{q}_{3, \mathbb{Z}}\end{array}\right]$ and for each $j$, with $1 \leq j \leq 3$, define $\boldsymbol{d}_{2, \mathbb{Z}}^{(j)}$ to be $\boldsymbol{d}_{2, \mathbb{Z}}$ with column $j$ removed.

(6) Define $\mathbb{F}_{\mathbb{Z}}$ to be the maps and modules

$\mathbb{F}_{\mathbb{Z}}: \quad 0 \rightarrow \boldsymbol{S}_{\mathbb{Z}}(-3 c,-3 c-3) \stackrel{\boldsymbol{d}_{3, \mathbb{Z}}}{\longrightarrow} \boldsymbol{S}_{\mathbb{Z}}(-3 c,-3 c-2)^{3} \stackrel{\boldsymbol{d}_{2, \mathbb{Z}}}{\longrightarrow} \boldsymbol{S}_{\mathbb{Z}}(-2 c,-1)^{3} \stackrel{\boldsymbol{d}_{1, \mathbb{Z}}}{\longrightarrow} \boldsymbol{S}_{\mathbb{Z}}$,

where $\boldsymbol{d}_{1, \mathbb{Z}}=\left[\begin{array}{lll}G_{1} & G_{2} & G_{3}\end{array}\right], \boldsymbol{d}_{2, \mathbb{Z}}$ is given in (5), and

$$
\boldsymbol{d}_{3, \mathbb{Z}}=\left[\begin{array}{c}
z_{0,1} \\
(-1)^{c+1} z_{0,2} \\
z_{0,3}
\end{array}\right] .
$$

Remark. If $\boldsymbol{k}$ is a field and $\boldsymbol{S}_{\mathbb{Z}}$ is the polynomial ring of Definition 5.21 , then $\boldsymbol{k} \otimes_{\mathbb{Z}} \boldsymbol{S}_{\mathbb{Z}}$ is equal to the polynomial $\operatorname{ring} \boldsymbol{S}=\boldsymbol{k}[x, y, \boldsymbol{z}]$ of Conventions 5.15.

Definition 5.23. Fix a field $\boldsymbol{k}$. Let $\Delta$ be the $\operatorname{ring} \boldsymbol{k} \otimes_{\mathbb{Z}} \Delta_{\mathbb{Z}}=\boldsymbol{S} /\left(G_{1}, G_{2}, G_{3}\right)$. If $s_{\mathbb{Z}}$ is an element of $S_{\mathbb{Z}}$, then write $s$ for the image of $s_{\mathbb{Z}}$ under the natural homomorphism $S_{\mathbb{Z}} \rightarrow S$; and if $M_{\mathbb{Z}}$ is a matrix with entries in $\boldsymbol{S}_{\mathbb{Z}}$, then write $M$ for the corresponding matrix with entries in $S$. In particular, the elements $w$ and $A(i, j)$ of $\boldsymbol{S}$ are obtained from the elements $w_{\mathbb{Z}}$ and $A_{\mathbb{Z}}(i, j)$ of $\boldsymbol{S}_{\mathbb{Z}}$ in this manner, the matrices $A^{(i)}, \boldsymbol{d}_{i}$, and $\boldsymbol{d}_{2}^{(j)}$, with entries in $\boldsymbol{S}$, are obtained from the matrices $A_{\mathbb{Z}}^{(i)}, \boldsymbol{d}_{i, \mathbb{Z}}$, and $\boldsymbol{d}_{2, \mathbb{Z}}^{(j)}$, with entries in $\boldsymbol{S}_{\mathbb{Z}}$, in this manner, and the maps and modules $\boldsymbol{k} \otimes_{\mathbb{Z}} \mathbb{F}_{\mathbb{Z}}$ are written as

$$
\mathbb{F}: \quad 0 \rightarrow \boldsymbol{S}(-3 c,-3 c-3) \stackrel{d_{3}}{\longrightarrow} \boldsymbol{S}(-3 c,-3 c-2)^{3} \stackrel{\boldsymbol{d}_{2}}{\longrightarrow} \boldsymbol{S}(-2 c,-1)^{3} \stackrel{\boldsymbol{d}_{1}}{\longrightarrow} \boldsymbol{S} .
$$

Theorem 5.24. Retain the notation and Conventions of 5.15. If $d=2 c$ is an even integer and $w$ in $\boldsymbol{R}$ is given in (3) of Definition 5.21 by way of Definition 5.23, then $\mathbb{A}_{d} \backslash \mathrm{Bal}_{d}$ is the closed subset $\left\{\boldsymbol{g} \in \mathbb{A}_{d} \mid \lambda_{\boldsymbol{g}} \in V(w)\right\}$ of $\mathbb{A}_{d}$.

Remark. It is an immediate consequence of Theorems 5.24 and 5.20 that $\mathrm{Bal}_{d}$ and $\mathbb{B}_{d}$ are open subsets of $\mathbb{A}_{d}$ and that $\mathbb{U B}_{d}$ is a hypersurface section of $\mathbb{T}_{d}$.

Proof. Recall $\rho_{\mathbb{Z}}^{(i)}, A_{\mathbb{Z}}^{(i)}, \boldsymbol{d}_{1, \mathbb{Z}}$, and $N_{\mathbb{Z}}^{(i)}$ from Definition 5.21. Matrix multiplication yields that

$$
\rho_{\mathbb{Z}}^{(d+i)} A_{\mathbb{Z}}^{(i)}=d_{1, \mathbb{Z}} N_{\mathbb{Z}}^{(i)},
$$

over $\boldsymbol{S}_{\mathbb{Z}}$, for each $i$. Let $\boldsymbol{q}$ be a $3 \times 1$ matrix of forms from $B=\boldsymbol{k}[x, y]$ of degree $i$. Then $\boldsymbol{q}=N^{(i)} \boldsymbol{b}$ for some $(3 i+3) \times 1$ matrix of scalars $\boldsymbol{b}$. Take the image of $(5.25)$ under the homomorphism $\boldsymbol{S}_{\mathbb{Z}} \rightarrow \boldsymbol{S}$ to see that

$$
\boldsymbol{d}_{1} \boldsymbol{q}=\boldsymbol{d}_{1} N^{(i)} \boldsymbol{b}=\rho^{(d+i)} A^{(i)} \boldsymbol{b}
$$


in $\boldsymbol{S}$. Fix $\boldsymbol{g}=\left(g_{1}, g_{2}, g_{3}\right) \in \mathbb{A}_{d}$. Apply the homomorphism $\boldsymbol{S} \rightarrow \boldsymbol{S}_{\lambda_{\boldsymbol{g}}}$ to (5.26) to see that

$$
\left[\begin{array}{lll}
g_{1} & g_{2} & g_{3}
\end{array}\right] \boldsymbol{q}=0 \text { in }\left.B \Longleftrightarrow A^{(i)}\right|_{\lambda_{\boldsymbol{g}}} \boldsymbol{b}=0 \text { in } \boldsymbol{k}
$$

Recall that $w$ is the determinant of the $3 c \times 3 c$ matrix $A^{(c-1)}$. Apply (5.27), with $i=c-1$, to see that

$\boldsymbol{g} \in \mathbb{A}_{d} \backslash \mathrm{Bal}_{d} \Longleftrightarrow$ there exists a non-zero $3 \times 1$ matrix $\boldsymbol{q}$ of forms of degree $c-1$ from $B$ with $\left[\begin{array}{lll}g_{1} & g_{2} & g_{3}\end{array}\right] \boldsymbol{q}=0$

$\Longleftrightarrow$ there exists a non-zero $3 c \times 1$ matrix $\boldsymbol{b}$ of constants with

$$
\left.A^{(c-1)}\right|_{\lambda_{\boldsymbol{g}}} \boldsymbol{b}=0
$$$$
\left.\Longleftrightarrow w\right|_{\lambda_{g}}=0 \text {. }
$$

Now that we have shown $\mathrm{Bal}_{d}$ and $\mathbb{B}_{d}$ to be open subsets of $\mathbb{A}_{d}$ it is time to show that the subsets $\mathrm{BalH}_{d}$ and $\mathbb{B H}_{d}$ of $\mathbb{H}_{d}$ are also open. These subsets were introduced in Definition 4.3.

Definition 5.28. Let $k$ be a field and $d=2 c$ be an even integer. Define

$$
\Phi: \mathbb{H}_{d} \rightarrow \mathbb{A}_{d}
$$

to be the morphism of affine varieties which sends a matrix $\varphi$ in $\mathbb{H}_{d}$ to the ordered triple $\Phi(\varphi)$ of signed maximal order minors of $\varphi$ as given in (4.5).

Observation 5.29. The subsets $\mathrm{BalH}_{d}$ and $\mathbb{B H}_{d}$ of $\mathbb{H}_{d}$ are open.

Proof. Observe that $\Phi^{-1}\left(\mathrm{Bal}_{d}\right)=\mathrm{BalH}_{d}$ and $\Phi^{-1}\left(\mathbb{B}_{d}\right)=\mathbb{B H}_{d}$. The subsets $\mathrm{Bal}_{d}$ and $\mathbb{B}_{d}$ of $\mathbb{A}_{d}$ are open by Theorem 5.24. The proof is complete because the morphism $\Phi$ is continuous.

Theorem 5.30. Retain the notation of Conventions 5.15 and let $\boldsymbol{d}_{2}^{(1)}, \boldsymbol{d}_{2}^{(2)}, \boldsymbol{d}_{2}^{(3)}$ be the matrices in $\boldsymbol{k}[\boldsymbol{z}, x, y]$ from (5) of Definition 5.21, by way of Definition 5.23. Then there exists an open cover $\mathrm{Bal}_{d}^{(1)} \cup \mathrm{Bal}_{d}^{(2)} \cup \mathrm{Bal}_{d}^{(3)}$ of $\mathrm{Bal}_{d}$ such that if $\boldsymbol{g} \in \mathrm{Bal}_{d}^{(j)}$, then $\left.\boldsymbol{d}_{2}^{(j)}\right|_{\lambda_{\boldsymbol{g}}}$ is a Hilbert-Burch matrix for $\boldsymbol{d}_{1}(\boldsymbol{g})$.

Remark. Define $\mathbb{B}_{d}^{(j)}=\mathrm{Bal}_{d}^{(j)} \cap \mathbb{B}_{d}$. Theorem 5.30 also asserts that there exists an open cover $\mathbb{B}_{d}^{(1)} \cup \mathbb{B}_{d}^{(2)} \cup \mathbb{B}_{d}^{(3)}$ of $\mathbb{B}_{d}$ such that if $\boldsymbol{g} \in \mathbb{B}_{d}^{(j)}$, then $\left.\boldsymbol{d}_{2}^{(j)}\right|_{\lambda_{\boldsymbol{g}}}$ is a Hilbert-Burch matrix for $\boldsymbol{d}_{1}(\boldsymbol{g})$. 
Proof. The set $U_{j}=\left\{\lambda \in \mathbb{A}^{3 d+3} \mid \lambda_{0, j} \neq 0\right\}$ is open in $\mathbb{A}^{3 d+3}$ for $1 \leq j \leq 3$; and therefore,

$$
\mathrm{Bal}_{d}^{(j)}=\mathrm{Bal}_{d} \cap U_{j}=\left\{\boldsymbol{g} \in \mathrm{Bal}_{d} \mid \lambda_{\boldsymbol{g}} \in U_{j}\right\}
$$

is open in $\mathrm{Bal}_{d}$. Furthermore, if $\boldsymbol{g} \in \mathrm{Bal}_{d}$, then the ideal $I_{\boldsymbol{g}}$ has height 2 by Observation 5.11; hence $I_{\boldsymbol{g}} \nsubseteq(x)$ and $\boldsymbol{g}$ is in $\mathrm{Bal}_{d}^{(j)}$ for some $j$. We have established that $\mathrm{Bal}_{d}^{(1)} \cup \mathrm{Bal}_{d}^{(2)} \cup \mathrm{Bal}_{d}^{(3)}$ is an open cover of $\mathrm{Bal}_{d}$.

The construction of $\boldsymbol{d}_{2, \mathbb{Z}}$ in Definition 5.21 guarantees that

$$
\boldsymbol{d}_{1, \mathbb{Z}} \boldsymbol{d}_{2, \mathbb{Z}}=0
$$

Indeed, the definition of $\boldsymbol{q}_{j, \mathbb{Z}}$ from (4) of Definition 5.21, together with (5.25) and the definition of $\boldsymbol{b}_{\mathbb{Z}}^{(j+(j-1) c)}$ from (3) of Definition 5.21, shows that

$$
\boldsymbol{d}_{1, \mathbb{Z}} \boldsymbol{q}_{j, \mathbb{Z}}=\boldsymbol{d}_{1, \mathbb{Z}} N_{\mathbb{Z}}^{(c)} \boldsymbol{b}_{\mathbb{Z}}^{(j+(j-1) c)}=\rho_{\mathbb{Z}}^{(d+c)} A_{\mathbb{Z}}^{(c)} \boldsymbol{b}_{\mathbb{Z}}^{(j+(j-1) c)}=0 .
$$

The equation (5.31) is preserved under the homomorphism $\boldsymbol{S}_{\mathbb{Z}} \rightarrow \boldsymbol{S}$; hence, the composition $\boldsymbol{d}_{1} \boldsymbol{d}_{2}$ is zero. Let $\boldsymbol{g} \in \mathbb{A}_{d}$. Evaluate $\boldsymbol{d}_{1} \boldsymbol{d}_{2}=0$ at $\lambda_{\boldsymbol{g}}$ to see that $\left.\boldsymbol{d}_{1}(\boldsymbol{g}) \boldsymbol{d}_{2}\right|_{\lambda_{\boldsymbol{g}}}=0$ and therefore, $\left.\boldsymbol{d}_{1}(\boldsymbol{g}) \boldsymbol{d}_{2}^{(j)}\right|_{\lambda_{\boldsymbol{g}}}=0$ for all $\boldsymbol{g} \in \mathbb{A}_{d}$ and all $j$.

Fix $\boldsymbol{g} \in \mathrm{Bal}_{d}^{(j)}$. We show that $\left.\boldsymbol{d}_{2}^{(j)}\right|_{\lambda_{\boldsymbol{g}}}$ is a Hilbert-Burch matrix for $\boldsymbol{d}_{1}(\boldsymbol{g})$. The fact that $\boldsymbol{g} \in \mathrm{Bal}_{d}^{(j)} \subseteq \mathrm{Bal}_{d}$ ensures that a minimal resolution of $\boldsymbol{R} / I_{\boldsymbol{g}}$ looks like

$$
0 \rightarrow \boldsymbol{R}(-3 c)^{2} \stackrel{\varphi}{\longrightarrow} \boldsymbol{R}(-2 c)^{3} \stackrel{\boldsymbol{d}_{1}(\boldsymbol{g})}{\longrightarrow} \boldsymbol{R} .
$$

To identify $\varphi$, we need only find two linearly independent degree $c$ relations on $\boldsymbol{d}_{1}(\boldsymbol{g})$. We already have seen that each of the two columns of $\left.\boldsymbol{d}_{2}^{(j)}\right|_{\lambda_{\boldsymbol{g}}}$ is a degree $c$ relation on $\boldsymbol{d}_{1}(\boldsymbol{g})$. To show that $\left.\boldsymbol{d}_{2}^{(j)}\right|_{\lambda_{\boldsymbol{g}}}$ is a Hilbert-Burch matrix for $\boldsymbol{d}_{1}(\boldsymbol{g})$, we need only see that the two columns of $\left.\boldsymbol{d}_{2}^{(j)}\right|_{\lambda_{\boldsymbol{g}}}$ are linearly independent. Look at $\boldsymbol{d}_{2, \mathbb{Z}}$ after $x$ has been set equal to zero:

$\boldsymbol{d}_{2, \mathbb{Z}} \equiv\left[\begin{array}{ccc}0 & A_{\mathbb{Z}}(1, c+2) y^{c} & A_{\mathbb{Z}}(1,2 c+3) y^{c} \\ (-1)^{c} A_{\mathbb{Z}}(1, c+2) y^{c} & 0 & (-1)^{c+1} A_{\mathbb{Z}}(c+2,2 c+3) y^{c} \\ -A_{\mathbb{Z}}(1,2 c+3) y^{c} & -A_{\mathbb{Z}}(c+2,2 c+3) y^{c} & 0\end{array}\right]$

$\bmod (x)$. Expand the minors $A_{\mathbb{Z}}(c+2,2 c+3), A_{\mathbb{Z}}(1,2 c+3)$, and $A_{\mathbb{Z}}(1, c+2)$ of $A_{\mathbb{Z}}$ across the first row to see that

$A_{\mathbb{Z}}(c+2,2 c+3)=z_{0,1} w_{\mathbb{Z}}, A_{\mathbb{Z}}(1,2 c+3)=(-1)^{c} z_{0,2} w_{\mathbb{Z}}$, and $A_{\mathbb{Z}}(1, c+2)=z_{0,3} w_{\mathbb{Z}}$. 
(Recall that $A_{\mathbb{Z}}$ is given in $(5.22)$ as $A_{\mathbb{Z}}^{(c)}$ and $w_{\mathbb{Z}}=\operatorname{det} A_{\mathbb{Z}}^{(c-1)}$ is defined in (2) of Definition 5.21.) Thus,

$$
\boldsymbol{d}_{2, \mathbb{Z}} \equiv y^{c}\left[\begin{array}{ccc}
0 & w_{\mathbb{Z}} z_{0,3} & (-1)^{c} w_{\mathbb{Z}} z_{0,2} \\
(-1)^{c} w_{\mathbb{Z}} z_{0,3} & 0 & (-1)^{c+1} w_{\mathbb{Z}} z_{0,1} \\
(-1)^{c+1} w_{\mathbb{Z}} z_{0,2} & -w_{\mathbb{Z}} z_{0,1} & 0
\end{array}\right] \quad \bmod (x) .
$$

We know from Theorem 5.24 and the definition of $\operatorname{Bal}_{d}^{(j)}$ that $\left.\left(w z_{0, j}\right)\right|_{\lambda_{\boldsymbol{g}}} \neq 0$. The matrix $\boldsymbol{d}_{2}^{(j)}$ is defined to be $\boldsymbol{d}_{2}$ with column $j$ removed. It is now clear that the columns of $\left.\boldsymbol{d}_{2}^{(j)}\right|_{\lambda_{\boldsymbol{g}}} \bmod (x)$ are linearly independent; hence, the columns of $\left.\boldsymbol{d}_{2}^{(j)}\right|_{\lambda_{\boldsymbol{g}}}$ are linearly independent and $\left.\boldsymbol{d}_{2}^{(j)}\right|_{\lambda_{\boldsymbol{g}}}$ is a Hilbert-Burch matrix for $\boldsymbol{d}_{1}(\boldsymbol{g})$.

Remark 5.33. Define $C^{(j)}$ and $A^{(j)}$ to be the bi-homogeneous matrices which satisfy (3.11) and (3.12) for $\boldsymbol{d}_{2}^{(j)}$. In other words, $C^{(j)}$ is a $(c+1) \times 2$ matrix and each entry is a bi-homogeneous element of $\boldsymbol{k}[\boldsymbol{z}, \boldsymbol{T}]$ of degree $3 c+1$ in $\boldsymbol{z}$ and degree 1 in $\boldsymbol{T}$. Furthermore, $A^{(j)}$ is a $(c+1) \times 3$ matrix and each entry is bi-homogeneous element in $\boldsymbol{k}[\boldsymbol{z}, \boldsymbol{u}]$ of degree $3 c+1$ in $\boldsymbol{z}$ and degree 1 in $\boldsymbol{u}$. These matrices satisfy

$$
\boldsymbol{T d}_{2}^{(j)}=\rho^{(c)} C^{(j)} \quad \text { and } \quad C^{(j)} \boldsymbol{u}^{\mathrm{T}}=A^{(j)} \boldsymbol{T}^{\mathrm{T}}
$$

Theorem 5.30 shows that if $\boldsymbol{g} \in \mathbb{B}_{d}^{(j)}$, then $\left.\boldsymbol{d}_{2}^{(j)}\right|_{\lambda_{\boldsymbol{g}}}$ is a Hilbert-Burch matrix for $\boldsymbol{d}_{1}(\boldsymbol{g})$; hence, the pair of matrices $\left(\left.C^{(j)}\right|_{\lambda_{\boldsymbol{g}}},\left.A^{(j)}\right|_{\lambda_{\boldsymbol{g}}}\right)$ complete the requirements of Data 3.10 for $\left.\boldsymbol{d}_{2}^{(j)}\right|_{\lambda_{\boldsymbol{g}}}$ and Corollary 3.23 may be applied to the ring $\boldsymbol{k}[\boldsymbol{u}] / I_{3}\left(\left.A^{(j)}\right|_{\lambda_{\boldsymbol{g}}}\right)$ in order to determine the configuration of multiplicity $c$ singularities on, or infinitely near, the curve $\mathcal{C}_{\boldsymbol{g}}$. This analysis is carried out in Theorem 6.10.

The rest of this section is devoted to giving alternate interpretations of Theorem 5.30. In Corollary 5.36 we prove that the maps and modules of $\mathbb{F}$, from Definition 5.23, behave as promised in (5.9). The ideas of flat families of algebras then allow us to prove, in Corollary 5.37, that the localization $\mathbb{F}_{w}$ is a resolution, at least when the ambient field is algebraically closed. In Theorem 5.41, we use Corollary 5.37, which holds over an algebraically closed field, together with an "evaluate the constant" argument, to identify the exact relationship, over $\mathbb{Z}$, between the maximal order minors of $\boldsymbol{d}_{2, \mathbb{Z}}^{(j)}$ and the "generic $d$-forms" $G_{1}, G_{2}, G_{3}$ in $\boldsymbol{S}_{\mathbb{Z}}=\mathbb{Z}[x, y, \boldsymbol{z}]$. One could obtain this result using identities involving determinants. In Theorem 5.41, we also prove that $\left(\mathbb{F}_{\mathbb{Z}}\right)_{w_{\mathbb{Z}}}$ is a resolution and that the ideal $\left(G_{1}, G_{2}, G_{3}\right)\left(S_{\mathbb{Z}}\right)_{w_{\mathbb{Z}}}$, which is defined over $\mathbb{Z}$, is perfect of grade 2 . At this point, one could recover Corollary 5.37 from Theorem 5.41 if one gives a direct argument for assertion (1) in Theorem 5.41 in place of the "evaluate the constant" argument that we give. 
Observation 5.34. The maps and modules of $\mathbb{F}_{\mathbb{Z}}$ from (6) of Definition 5.21 are a bi-homogeneous complex of $\boldsymbol{S}_{\mathbb{Z}}$-modules.

Remark. It follows that $\mathbb{F}$ from Definition 5.23 is a bi-homogeneous complex of free $S$-modules.

Proof. We saw in (5.31) that the composition $\boldsymbol{d}_{1, \mathbb{Z}} \boldsymbol{d}_{2, \mathbb{Z}}$ is zero. The Eagon-Northcott complex which is associated to the map $A_{\mathbb{Z}}=A_{\mathbb{Z}}^{(c)}$ of (5.22) looks like :

$$
0 \rightarrow \boldsymbol{R}_{\mathbb{Z}}^{3 c+1} \otimes \bigwedge^{3 c+3} \boldsymbol{R}_{\mathbb{Z}}^{3 c+3} \rightarrow \bigwedge^{3 c+2} \boldsymbol{R}_{\mathbb{Z}}^{3 c+3} \rightarrow \boldsymbol{R}_{\mathbb{Z}}^{3 c+3} \stackrel{A}{\rightarrow} \boldsymbol{R}_{\mathbb{Z}}^{3 c+1}
$$

Each row of $A_{\mathbb{Z}}$ gives rise to a relation on the $3 c+3$ Eagon-Northcott relations on $A_{\mathbb{Z}}$. In particular, the top row of $A_{\mathbb{Z}}$ gives rise to the relation

$$
z_{0,1} \boldsymbol{b}_{\mathbb{Z}}^{(1)}+(-1)^{c+1} z_{0,2} \boldsymbol{b}_{\mathbb{Z}}^{(c+2)}+z_{0,3} \boldsymbol{b}_{\mathbb{Z}}^{(2 c+3)}=0
$$

for $\boldsymbol{b}_{\mathbb{Z}}^{(j)}$ as defined in (3) of Definition 5.21. Multiply (5.35) by $N_{\mathbb{Z}}^{(c)}$ and use (4) of Definition 5.21 to see that $\boldsymbol{d}_{3, \mathbb{Z}}$ is a non-trivial relation on $\boldsymbol{d}_{2, \mathbb{Z}}$.

Corollary 5.36. Retain the notation and hypotheses of Conventions 5.15 and let $\mathbb{F}$ be the complex of free $S$-modules which is defined in Definition 5.23. Then $\mathbb{F} \otimes_{\boldsymbol{R}} \boldsymbol{R}_{\lambda_{\boldsymbol{g}}}$ is a resolution of $B / I_{\boldsymbol{g}}$ for all $\boldsymbol{g} \in \mathrm{Bal}_{d}$.

Remark. If $\boldsymbol{g} \in \mathbb{A}_{d} \backslash \mathrm{Bal}_{d}$, then the complex $\mathbb{F} \otimes_{\boldsymbol{R}} \boldsymbol{R}_{\lambda_{g}}$ is not a resolution of $B / I_{\boldsymbol{g}}$ because it has the wrong graded Betti numbers. Thus, this Corollary establishes the claim made in (5.9).

Proof. We saw in Theorem 5.30 that $\left\{\mathrm{Bal}_{d}^{(j)}\right\}$ is an open cover of $\mathrm{Bal}_{d}$. Fix an element $\boldsymbol{g}$ of $\mathrm{Bal}_{d}^{(j)}$. Theorem 5.30 also shows that

$$
0 \rightarrow B(-3 c)^{2} \stackrel{\left.\boldsymbol{d}_{2}^{(j)}\right|_{\lambda_{\boldsymbol{g}}}}{\longrightarrow} B(-2 c)^{3} \stackrel{\boldsymbol{d}_{1}(\boldsymbol{g})}{\longrightarrow} B
$$

is a resolution of $B / I_{\boldsymbol{g}}$. Observe that $\boldsymbol{d}_{1} \otimes_{\boldsymbol{R}} \boldsymbol{R}_{\lambda_{\boldsymbol{g}}}=\boldsymbol{d}_{1}(\boldsymbol{g})$, two of the columns of $\boldsymbol{d}_{2} \otimes_{\boldsymbol{R}} \boldsymbol{R}_{\lambda_{\boldsymbol{g}}}$ form $\left.\boldsymbol{d}_{2}^{(j)}\right|_{\lambda_{\boldsymbol{g}}}$, and $\boldsymbol{d}_{3} \otimes_{\boldsymbol{R}} \boldsymbol{R}_{\lambda_{\boldsymbol{g}}}$ is the relation on the columns of $\boldsymbol{d}_{2} \otimes_{\boldsymbol{R}} \boldsymbol{R}_{\lambda_{\boldsymbol{g}}}$ which expresses the redundant column in terms of the columns of $\left.\boldsymbol{d}_{2}^{(j)}\right|_{\lambda_{\boldsymbol{g}}}$. In other words, $\mathbb{F} \otimes_{\boldsymbol{R}} \boldsymbol{R}_{\lambda_{\boldsymbol{g}}}$ is a (non-minimal) resolution of $B / I_{\boldsymbol{g}}$.

Corollary 5.37. Retain the notation and hypotheses of Conventions 5.15 with $\boldsymbol{k}$ an algebraically closed field. Let $w, \mathbb{F}$, and $\Delta$ be the element of $\boldsymbol{S}$, the complex 
of free $\boldsymbol{S}$-modules, and the quotient ring of $\boldsymbol{S}$ which are defined in Definition 5.23. Then the following statements hold.

(1) The ring homomorphism $\boldsymbol{R}_{w} \rightarrow \Delta_{w}$, which is the composition

$$
\boldsymbol{R}_{w} \rightarrow \boldsymbol{S}_{w} \rightarrow \Delta_{w}
$$

of inclusion followed by the natural quotient map, is flat.

(2) The ring $\Delta_{w}$ is Cohen-Macaulay.

(3) The complex $\mathbb{F}_{w}$ is a resolution of $\boldsymbol{\Delta}_{w}$ by free $\boldsymbol{S}_{w}$-modules.

Proof. Recall the maximal ideal $\mathfrak{m}_{\lambda}=\left(\left\{z_{i, j}-\lambda_{i, j}\right\}\right)$ of $\boldsymbol{R}$ which is defined in (2) of Conventions 5.15. The field $\boldsymbol{k}$ is algebraically closed; so MaxSpec $\boldsymbol{R}_{w}$ is identified with $\mathbb{A}^{3 d+3} \backslash V(w)$. To show assertion (1) it suffices to show that the ring homomorphism $\boldsymbol{R}_{\mathfrak{m}_{\lambda}} \rightarrow \Delta_{\mathfrak{m}_{\lambda}}$ is flat for all $\mathfrak{m}_{\lambda}$ in MaxSpec $\boldsymbol{R}_{w}$. Fix one such $\mathfrak{m}_{\lambda}$. The ring $\Delta_{\mathfrak{m}_{\lambda}}$ is equal to $\boldsymbol{R}_{\mathfrak{m}_{\lambda}}[x, y] /\left(G_{1}, G_{2}, G_{3}\right)$; and therefore, $\mathfrak{m}_{\lambda} \Delta_{\mathfrak{m}_{\lambda}}$ is contained in the Jacobson radical of $\Delta_{\mathfrak{m}_{\lambda}}$. We apply the local criterion for flatness, (see, for example, [27, Thm. 49]). To show that $\boldsymbol{R}_{\mathfrak{m}_{\lambda}} \rightarrow \Delta_{\mathfrak{m}_{\lambda}}$ is flat it suffices to prove that $\operatorname{Tor}_{1}^{\boldsymbol{R}_{\mathfrak{m}_{\lambda}}}\left(\boldsymbol{R}_{\lambda}, \Delta_{\mathfrak{m}_{\lambda}}\right)=0$ (since $\boldsymbol{R}_{\lambda}$ means $\left.\boldsymbol{R}_{\mathfrak{m}_{\lambda}} / \mathfrak{m}_{\lambda} \boldsymbol{R}_{\mathfrak{m}_{\lambda}}\right)$. Consider a resolution $\mathbb{G}$ of $\Delta_{\mathfrak{m}_{\lambda}}$ by free $R_{\mathfrak{m}_{\lambda}}[x, y]$-modules. One can create $\mathbb{G}$ by starting with $\mathbb{F} \otimes_{\boldsymbol{R}} \boldsymbol{R}_{\mathfrak{m}_{\lambda}}$ and adjoining extra summands in positions 2 and higher. We saw in Corollary 5.36 that $\mathrm{H}_{1}\left(\mathbb{F} \otimes_{\boldsymbol{R}} \boldsymbol{R}_{\mathfrak{m}_{\lambda}} \otimes_{\boldsymbol{R}_{\mathfrak{m}_{\lambda}}} \boldsymbol{R}_{\lambda}\right)=0$. It follows that

$$
0=\mathrm{H}_{1}\left(\mathbb{G} \otimes_{\boldsymbol{R}_{\mathfrak{m}_{\lambda}}} \boldsymbol{R}_{\lambda}\right)=\operatorname{Tor}_{1}^{\boldsymbol{R}_{\mathfrak{m}_{\lambda}}}\left(\boldsymbol{R}_{\lambda}, \Delta_{\mathfrak{m}_{\lambda}}\right)
$$

and (1) is established.

The field $k$ remains algebraically closed; so, the maximal ideals of $\Delta$ continue to correspond to points in $\mathbb{A}^{3 d+3} \times \mathbb{P}^{1}$. If $\mathfrak{M}$ is a maximal ideal of $\Delta$, then $\mathfrak{M} \cap \boldsymbol{R}_{w}$ is a maximal ideal $\mathfrak{m}_{\lambda}$ of $R$ and $\boldsymbol{R}_{\mathfrak{m}_{\lambda}} \rightarrow \Delta_{\mathfrak{M}}$ is a flat local homomorphism. The base ring $\boldsymbol{R}_{\mathfrak{m}_{\lambda}}$ and the local ring of the fiber $\Delta_{\mathfrak{M}} \otimes_{\boldsymbol{R}_{\mathfrak{m}_{\lambda}}} \boldsymbol{R}_{\lambda}=B / I_{\boldsymbol{g}_{\lambda}}$ are both Cohen-Macaulay. It follows (see, for example, [27, Thm. 50]) that $\Delta_{\mathfrak{M}}$ is a Cohen-Macaulay ring of dimension

$$
\operatorname{dim} \boldsymbol{R}_{\mathfrak{m}_{\lambda}}+\operatorname{dim} B / I_{\boldsymbol{g}_{\lambda}}=3 d+3
$$

and (2) is established.

Consider the augmented complex $\mathbb{F}_{w}^{\text {aug }}: 0 \rightarrow \mathbb{F}_{w} \rightarrow \Delta \rightarrow 0$. Fix a maximal ideal $\mathfrak{M}$ of $\boldsymbol{S}_{w}$. Let $\mathbb{F}_{\mathfrak{M}}^{\text {aug }}$ denote the localization of $\mathbb{F}_{w}^{\text {aug }}$ at $\mathfrak{M}$ and let $\mathfrak{m}_{\lambda}$ denote $\mathfrak{M} \cap \boldsymbol{R}_{w}$. The generators $\left\{z_{i, j}-\lambda_{i, j}\right\}$ of $\mathfrak{m}_{\lambda}$ are a regular sequence on $\boldsymbol{S}_{w}$ and they form a system of parameters on the Cohen-Macaulay ring $\Delta$; hence, $\left\{z_{i, j}-\lambda_{i, j}\right\}$ is a regular sequence on each non-zero module in $\mathbb{F}_{\mathfrak{M}}^{\text {aug }}$. Apply Lemma 5.39 to see that

$$
\mathbb{F}_{\mathfrak{M}}^{\text {aug }} \text { is exact } \Longleftrightarrow \mathbb{F}_{\mathfrak{M}}^{\text {aug }} \otimes_{\boldsymbol{S}_{\mathfrak{M}}} \boldsymbol{S}_{\mathfrak{M}} / \mathfrak{m}_{\lambda} \text { is exact. }
$$


The augmented complex on the right hand side of (5.38) is the localization

$$
\left(\mathbb{F} \otimes_{\boldsymbol{R}} \boldsymbol{R}_{\lambda_{\boldsymbol{g}}} \rightarrow B / I_{\boldsymbol{g}_{\lambda}}\right)_{\mathfrak{M}}
$$

of $\mathbb{F} \otimes_{\boldsymbol{R}} \boldsymbol{R}_{\lambda_{\boldsymbol{g}}} \rightarrow B / I_{\boldsymbol{g}_{\lambda}}$, which is exact by (2), and the proof of (3) is complete.

We used one direction of the following result in the course of proving Corollary 5.37 .

Lemma 5.39. Let $R$ be a local ring and

$$
\mathbb{F}: \quad \cdots \stackrel{d_{2}}{\longrightarrow} F_{1} \stackrel{d_{1}}{\longrightarrow} F_{0} \rightarrow 0
$$

be a complex of $R$-modules. Suppose that $z_{1}, \ldots, z_{r}$ are elements of $R$ which form a regular sequence on each non-zero module in $\mathbb{F}$. Then $\mathbb{F}$ is exact if and only if $\mathbb{F} \otimes_{R} R /\left(z_{1}, \ldots, z_{r}\right)$ is exact

Proof. By induction it suffices to prove the result when $r=1$. Write $z$ for $z_{1}$. $(\Rightarrow)$ Assume that $\mathbb{F}$ is exact. Decompose $\mathbb{F}$ into a collect of of short exact sequences:

$$
0 \rightarrow B_{1} \rightarrow F_{1} \rightarrow F_{0} \rightarrow 0, \quad 0 \rightarrow B_{2} \rightarrow F_{2} \rightarrow B_{1} \rightarrow 0, \quad \ldots,
$$

where $B_{i}=\operatorname{im} d_{i+1}$. Observe that $z$ is regular on each $B_{i}$. Apply $\otimes_{R} R /(z)$ and glue the resulting short exact sequences back together to form the exact sequence $\mathbb{F} \otimes_{R} R /(z)$.

$(\Leftarrow)$ Let $x$ be a cycle in $F_{i}$. The hypothesis that $\mathbb{F} \otimes_{R} R /(z)$ is exact ensures that $x=b+z x_{2}$ for some $b \in B_{i}$ and $x_{2} \in F_{i}$. The hypothesis that $z$ is regular on $F_{i}$ yields that $x_{2}$ is also a cycle. Repeat this process to see that $z$ is in $\cap_{n}\left(B_{i}+z^{n} F_{i}\right)$, and this module, by the Krull Intersection Theorem, is $B_{i}$.

On at least three occasions, we apply the Buchsbaum-Eisenbud acyclicity criterion; furthermore, we apply the criterion in both directions. Roughly speaking, this criterion states that if

$$
F_{\bullet}: \quad 0 \rightarrow F_{s} \stackrel{\varphi_{s}}{\longrightarrow} \cdots \stackrel{\varphi_{1}}{\longrightarrow} F_{0}
$$

is a complex of finitely generated free modules over the Noetherian ring $R$, then $F_{\text {• }}$ is acyclic if and only if grade $I_{r_{i}}\left(\varphi_{i}\right) \geq i$ (or $I_{r_{i}}\left(\varphi_{i}\right)=R$ ) for $1 \leq i \leq s$, where $r_{i}$ is the expected rank of $\varphi_{i}$. More details may be found in [6], [11, Thm. 20.9], or [4, Thm. 1.4.12], and many other places.

Recall the function $\Phi$ :

$$
\left[\begin{array}{ll}
Q_{1,1} & Q_{1,2} \\
Q_{2,1} & Q_{2,2} \\
Q_{3,1} & Q_{3,2}
\end{array}\right] \stackrel{\Phi}{\rightarrow}\left[\left|\begin{array}{ll}
Q_{2,1} & Q_{2,2} \\
Q_{3,1} & Q_{3,2}
\end{array}\right|,-\left|\begin{array}{ll}
Q_{1,1} & Q_{1,2} \\
Q_{3,1} & Q_{3,2}
\end{array}\right|,\left|\begin{array}{ll}
Q_{1,1} & Q_{1,2} \\
Q_{2,1} & Q_{2,2}
\end{array}\right|\right]
$$

which sends a $3 \times 2$ matrix to a $1 \times 3$ matrix of signed $2 \times 2$ minors. 
Theorem 5.41. Adopt the notation of Definition 5.21. Let $\Phi$ be the function defined in (5.40). Then the following statements hold.

(1) The row vector $\Phi\left(\boldsymbol{d}_{2, \mathbb{Z}}^{(j)}\right)+(-1)^{j c} z_{0, j} w_{\mathbb{Z}}^{2} \boldsymbol{d}_{1, \mathbb{Z}}$, with entries in $\boldsymbol{S}_{\mathbb{Z}}$, is identically zero, for $1 \leq j \leq 3$.

(2) The complex $\left(\mathbb{F}_{\mathbb{Z}}\right)_{w_{\mathbb{Z}}}$ is a resolution of $\left(\Delta_{\mathbb{Z}}\right)_{w_{\mathbb{Z}}}$ by free $\left(\boldsymbol{S}_{\mathbb{Z}}\right)_{w_{\mathbb{Z}}}$-modules.

(3) The ideal $\left(G_{1}, G_{2}, G_{3}\right)$ of $\left(\boldsymbol{S}_{\mathbb{Z}}\right)_{w_{\mathbb{Z}}}$ is perfect of grade two.

(4) The complex

$$
\mathbb{F}_{\mathbb{Z}}^{(j)}: \quad 0 \rightarrow\left(\boldsymbol{S}_{\mathbb{Z}}\right)_{w_{\mathbb{Z}} z_{0, j}}^{2} \stackrel{\boldsymbol{d}_{2, \mathbb{Z}}^{(j)}}{\longrightarrow}\left(\boldsymbol{S}_{\mathbb{Z}}\right)_{w_{\mathbb{Z}} z_{0, j}}^{3} \stackrel{\boldsymbol{d}_{1, \mathbb{Z}}}{\longrightarrow}\left(\boldsymbol{S}_{\mathbb{Z}}\right)_{w_{\mathbb{Z}} z_{0, j}}
$$

is a resolution of $\Delta_{w z_{0, j}}$ by free $\left(\boldsymbol{S}_{\mathbb{Z}}\right)_{w_{\mathbb{Z}} z_{0, j}}$-modules for $1 \leq j \leq 3$.

Proof. Let $\boldsymbol{k}$ be an algebraically closed field of characteristic zero. The natural ring homomorphism $S_{\mathbb{Z}} \rightarrow \boldsymbol{k} \otimes_{\mathbb{Z}} \boldsymbol{S}_{\mathbb{Z}}=S$ is an injection. Each element in each matrix in (1) is an element of $\boldsymbol{S}_{\mathbb{Z}}$. If the left side of (1) is sent to zero in $\boldsymbol{S}$, then the left side of (1) is already zero in $\boldsymbol{S}_{\mathbb{Z}}$. We make our calculation in $\boldsymbol{S}$. Recall the convention of Definition 5.23: if $s_{\mathbb{Z}}$ is an element of $\boldsymbol{S}_{\mathbb{Z}}$, then the image of $s_{\mathbb{Z}}$ in $\boldsymbol{S}$ is denoted by $s$. We saw in Corollary 5.37 that $\mathbb{F}_{w}$ is a resolution of $\Delta_{w}$ by free $S_{w}$-modules. Fix $j$. We localize further to see that $\mathbb{F}_{w z_{0, j}}$ is a resolution of $\Delta_{w z_{0, j}}$ by free $\boldsymbol{S}_{w z_{0, j}}$-modules. One entry of $\boldsymbol{d}_{3}$ is a unit of $S_{w z_{0, j}}$. We split off a rank one summand from positions 2 and 3 of $\mathbb{F}_{w z_{0, j}}$ to obtain the resolution

$$
0 \rightarrow \boldsymbol{S}_{w z_{0, j}}^{2} \stackrel{\boldsymbol{d}_{2}^{(j)}}{\longrightarrow} \boldsymbol{S}_{w z_{0, j}}^{3} \stackrel{\boldsymbol{d}_{1}}{\longrightarrow} \boldsymbol{S}_{w z_{0, j}} \rightarrow \Delta_{w z_{0, j}} \rightarrow 0
$$

The Hilbert-Burch Theorem (or the Buchsbaum-Eisenbud acyclicity criterion) ensures that the maximal order minors of $\boldsymbol{d}_{2}^{(j)}$ generate a grade two ideal of $S_{w z_{0, j}}$; hence,

$$
0 \rightarrow \boldsymbol{S}_{w z_{0, j}} \stackrel{\Phi\left(\boldsymbol{d}_{2}^{(j)}\right)^{\mathrm{T}}}{\longrightarrow} \boldsymbol{S}_{w z_{0, j}}^{3} \stackrel{\boldsymbol{d}_{2}^{(j)^{\mathrm{T}}}}{\longrightarrow} \boldsymbol{S}_{w z_{0, j}}^{2}
$$

is acyclic. Of course, $\boldsymbol{d}_{1}^{\mathrm{T}}$ is in the kernel of $\boldsymbol{d}_{2}^{(j)^{\mathrm{T}}}$. It follows that $\boldsymbol{d}_{1}=\theta \Phi\left(\boldsymbol{d}_{2}^{(j)}\right)$, for some $\theta$ in $\boldsymbol{S}_{w z_{0, j}}$. View $\boldsymbol{S}_{w z_{0, j}}$ as the polynomial ring $\boldsymbol{R}_{w z_{0, j}}[x, y]$ where each element of $\boldsymbol{R}_{w z_{0, j}}$ has degree zero and $x$ and $y$ have degree 1 . The entries of $\boldsymbol{d}_{1}$ and the entries of $\Phi\left(\boldsymbol{d}_{2}^{(j)}\right)$ are homogeneous forms of degree $d$; hence, $\theta$ is in $\boldsymbol{R}_{w z_{0, j}}$. We identify $\theta$ by looking at the coefficient of $y^{d}$ in position $j$. In $\boldsymbol{d}_{1}$, this coefficient is $z_{0, j}$. In $\Phi\left(\boldsymbol{d}_{2}^{(j)}\right)$, this coefficient may easily be read from (5.32); it is

$$
\left\{\begin{array}{cc}
\left|\begin{array}{cc}
0 & (-1)^{c+1} w z_{0,1} \\
-w z_{0,1} & 0
\end{array}\right| & \text { if } j=1 \\
-\left|\begin{array}{cc}
0 & (-1)^{c} w z_{0,2} \\
(-1)^{c+1} w z_{0,2} & 0
\end{array}\right| & \text { if } j=2 \\
\begin{array}{ll}
0 & w z_{0,3} \\
(-1)^{c} w z_{0,3} & 0
\end{array} \mid & \text { if } j=3 .
\end{array}\right.
$$


In other words, in $\Phi\left(\boldsymbol{d}_{2}^{(j)}\right)$, this coefficient is $(-1)^{j c+1} w^{2} z_{0, j}^{2}$. It follows that $\theta$ is equal to $\frac{(-1)^{j c+1}}{w^{2} z_{0, j}}$ and the proof of $(1)$ is complete.

We make two observations before proving (2). First of all, the polynomials $G_{1}, G_{2}$ in $\boldsymbol{S}_{\mathbb{Z}}$ are generic linear combinations of the generators of the grade 2 ideal $(x, y)^{d}$ of $\boldsymbol{k}[x, y]$; and therefore,

$$
G_{1}, G_{2} \text { form a regular sequence on } S_{\mathbb{Z}}
$$

see, for example, [18]. Secondly, $w_{\mathbb{Z}}$ is the determinant of the matrix $A_{\mathbb{Z}}^{(c-1)}$ of (5.22). One can expand $\operatorname{det} A_{\mathbb{Z}}^{(c-1)}$ across the first row and write

$$
w_{\mathbb{Z}}=z_{0,1} Z_{1, \mathbb{Z}}+z_{0,2} Z_{2, \mathbb{Z}}+z_{0,3} Z_{3, \mathbb{Z}}
$$

where $Z_{1, \mathbb{Z}}, Z_{2, \mathbb{Z}}$, and $Z_{3, \mathbb{Z}}$ are the appropriate signed minors of $A_{\mathbb{Z}}^{(c-1)}$.

To prove (2) it suffices to show that the localization of $\mathbb{F}_{\mathbb{Z}}$ at $P$ is exact for all prime ideals $P$ of $\left(\boldsymbol{S}_{\mathbb{Z}}\right)_{w_{\mathbb{Z}}}$. If $P$ is such a prime ideal, then $w_{\mathbb{Z}} \notin P$ and (5.43) shows that some $z_{0, j} \notin P$. Consequently, to prove $(2)$, it suffices to show that $\left(\mathbb{F}_{\mathbb{Z}}\right)_{w_{\mathbb{Z}} z_{0, j}}$ is exact for all $j$. We employ the Buchsbaum-Eisenbud acyclicity criterion once again. We see that $I_{1}\left(\left(\boldsymbol{d}_{3, \mathbb{Z}}\right)_{w_{\mathbb{Z}} z_{0, j}}\right)$ is the entire ring $\left(S_{\mathbb{Z}}\right)_{w_{\mathbb{Z}} z_{0, j}}$. Assertion (1) yields that $G_{1}$ and $G_{2}$ are in $I_{2}\left(\left(d_{2, \mathbb{Z}}\right)_{w_{\mathbb{Z}} z_{0, j}}\right)$; and therefore (5.42) shows that the grade of $I_{2}\left(\left(d_{2, \mathbb{Z}}\right)_{w_{\mathbb{Z}} z_{0, j}}\right)$ is at least 2 . The proof of $(2)$ is complete.

The inequality

$$
\operatorname{grade}\left(G_{1}, G_{2}, G_{3}\right)\left(\boldsymbol{S}_{\mathbb{Z}}\right)_{w_{\mathbb{Z}}} \leq \operatorname{pd}_{\left(\boldsymbol{S}_{\mathbb{Z}}\right)_{w_{\mathbb{Z}}}}\left(\Delta_{\mathbb{Z}}\right)_{w_{\mathbb{Z}}}
$$

holds automatically. In (3) we assert that both numbers are equal to 2 . See, for example, [4, pg. 25] for more information about perfect modules. The observation (5.42) gives $2 \leq \operatorname{grade}\left(G_{1}, G_{2}, G_{3}\right)\left(S_{\mathbb{Z}}\right)_{w_{\mathbb{Z}}}$. On the other hand, we saw in (2) that

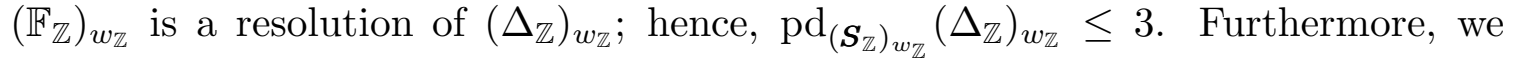
know an explicit splitting map for

$$
\left(\boldsymbol{S}_{\mathbb{Z}}\right)_{w_{\mathbb{Z}}} \stackrel{\boldsymbol{d}_{3, \mathbb{Z}}}{\longrightarrow}\left(\boldsymbol{S}_{\mathbb{Z}}\right)_{w_{\mathbb{Z}}}^{3}
$$

Define

$$
\sigma_{\mathbb{Z}}:\left(\boldsymbol{S}_{\mathbb{Z}}\right)_{w_{\mathbb{Z}}}^{3} \rightarrow\left(\boldsymbol{S}_{\mathbb{Z}}\right)_{w_{\mathbb{Z}}} \text { to be } \frac{1}{w_{\mathbb{Z}}}\left[\begin{array}{lll}
Z_{1, \mathbb{Z}} & Z_{2, \mathbb{Z}} & Z_{3, \mathbb{Z}}
\end{array}\right]
$$

We see that $\sigma_{\mathbb{Z}} \circ \boldsymbol{d}_{3, \mathbb{Z}}$ is the identity on $\left(\boldsymbol{S}_{\mathbb{Z}}\right)_{w_{\mathbb{Z}}}$. It follows that $\left(\boldsymbol{S}_{\mathbb{Z}}\right)_{w_{\mathbb{Z}}}^{3}$ is equal to $\operatorname{im} \boldsymbol{d}_{3, \mathbb{Z}} \oplus \operatorname{ker} \sigma_{\mathbb{Z}}$ and that

$$
\mathbb{U} \mathbb{R}_{\mathbb{Z}}: \quad 0 \rightarrow \operatorname{ker} \sigma_{\mathbb{Z}} \stackrel{\boldsymbol{d}_{2, \mathbb{Z}} \mid \operatorname{ker} \sigma_{\mathbb{Z}}}{\longrightarrow}\left(\boldsymbol{S}_{\mathbb{Z}}\right)_{w_{\mathbb{Z}}}^{3} \stackrel{\boldsymbol{d}_{1, \mathbb{Z}}}{\longrightarrow}\left(\boldsymbol{S}_{\mathbb{Z}}\right)_{w_{\mathbb{Z}}} \rightarrow\left(\Delta_{\mathbb{Z}}\right)_{w_{\mathbb{Z}}} \rightarrow 0
$$


is a length 2 projective resolution of $\left(\Delta_{\mathbb{Z}}\right)_{w_{\mathbb{Z}}}$ and the proof of $(3)$ is complete.

Assertion (1) shows that $\left(G_{1}, G_{2}\right) \subseteq I_{2}\left(\boldsymbol{d}_{2, \mathbb{Z}}^{(j)}\right)\left(\boldsymbol{S}_{\mathbb{Z}}\right)_{w_{\mathbb{Z}} z_{0, j}}$; so, (5.42) gives

$$
2 \leq \operatorname{grade} I_{2}\left(\boldsymbol{d}_{2, \mathbb{Z}}^{(j)}\right)\left(\boldsymbol{S}_{\mathbb{Z}}\right)_{w_{\mathbb{Z}} z_{0, j}}
$$

and (4) follows from the Buchsbaum-Eisenbud acyclicity criterion (or the HilbertBurch Theorem).

Corollary 5.45. Let $\boldsymbol{k}$ be a field, $d=2 c$ be an even integer, $\Phi: \mathbb{H}_{d} \rightarrow \mathbb{A}_{d}$ be the morphism which sends a $3 \times 2$ matrix with entries from $\boldsymbol{k}[x, y]_{c}$ to a triple $\boldsymbol{g}$ of $\mathbb{A}_{d}$, and let $\Phi \mid$ be the restriction

$$
\Phi \mid: \Phi^{-1}\left(\mathrm{Bal}_{d}\right) \rightarrow \mathrm{Bal}_{d}
$$

of $\Phi$ to $\Phi^{-1}\left(\mathrm{Bal}_{d}\right)$. Then there exists a local section of the morphism $\Phi \mid$ of (5.10). Proof. We saw in Theorem 5.30 that $\cup_{j=1}^{3} \mathrm{Bal}_{d}^{(j)}$ is an open cover of $\mathrm{Bal}_{d}$. Consider $w$ and $\boldsymbol{d}_{2}^{(j)}$ as found in Definition 5.23. Let $D^{(j)}$ be the matrix whose first column is $\frac{(-1)^{j c+1}}{w^{2} z_{0, j}}$ times the first column of $\boldsymbol{d}_{2}^{(j)}$ and whose second column is the second column of $\boldsymbol{d}_{2}^{(j)}$. Define $\sigma_{j}: \mathrm{Bal}_{d}^{(j)} \rightarrow \mathbb{H}_{d}$ by

$$
\left.\boldsymbol{g} \mapsto\left[D^{(j)}\right]\right|_{\lambda_{\boldsymbol{g}}}
$$

for $\boldsymbol{g} \in \mathrm{Bal}_{d}^{(j)}$. Assertion (1) of Theorem 5.41 shows that $\Phi \mid \circ \sigma_{j}$ is the identity morphism on $\mathrm{Bal}_{d}^{(j)}$.

Corollary 5.46. Let $\boldsymbol{k}$ be a field, $B$ be the standard graded polynomial ring $\boldsymbol{k}[x, y]$ and $c$ be a positive integer. Then the projective resolution $\mathbb{U} \mathbb{R}_{\mathbb{Z}}$ of $(5.44)$ is a universal projective resolution for the graded Betti numbers

$$
0 \rightarrow B(-3 c)^{2} \rightarrow B(-2 c)^{3} \rightarrow B \rightarrow B / I \rightarrow 0
$$

Proof. Let $I$ be a homogeneous ideal of $B$ with the property that the graded Betti numbers of $B / I$ are given by (5.47). In particular, $I$ is generated by three forms $g_{1}, g_{2}, g_{3}$ of degree $2 c$. Let $d$ be the integer $2 c$ and $\boldsymbol{g}$ be the triple $\left(g_{1}, g_{2}, g_{3}\right)$ of $\mathbb{A}_{d}$. In the language of Definition 5.3, we have $I_{\boldsymbol{g}}=I$. The hypothesis (5.47) also shows that $\boldsymbol{g} \in \mathrm{Bal}_{d}$. It follows from Theorem 5.24 that $\lambda_{\boldsymbol{g}} \notin V(w)$; and therefore, $\boldsymbol{R}_{\lambda_{\boldsymbol{g}}}$ is an $\boldsymbol{R}_{w^{-}}$-algebra. Consider the homomorphism $\alpha:\left(\boldsymbol{R}_{\mathbb{Z}}\right)_{w_{\mathbb{Z}}} \rightarrow \boldsymbol{R}_{\lambda_{\boldsymbol{g}}}$ which is the composition of the natural maps

$$
\left(\boldsymbol{R}_{\mathbb{Z}}\right)_{w_{\mathbb{Z}}} \rightarrow\left(\boldsymbol{R}_{\mathbb{Z}}\right)_{w_{\mathbb{Z}}} \otimes_{\mathbb{Z}} \boldsymbol{k}=\boldsymbol{R}_{w} \quad \text { and } \quad \boldsymbol{R}_{w} \rightarrow \frac{\boldsymbol{R}_{w}}{\left(\mathfrak{m}_{\lambda_{\boldsymbol{g}}}\right) \boldsymbol{R}_{w}}=\boldsymbol{R}_{\lambda_{\boldsymbol{g}}}
$$


We establish the result by showing that $U \mathbb{P} \mathbb{R}_{\mathbb{Z}} \otimes_{\left(\boldsymbol{R}_{\mathbb{Z}}\right)_{w_{\mathbb{Z}}}} \boldsymbol{R}_{\lambda_{\boldsymbol{g}}}$ is a resolution of $B / I$ by free $B$-modules with graded Betti numbers given by (5.47). Corollary 5.36 shows that $\mathbb{F} \otimes_{\boldsymbol{R}} \boldsymbol{R}_{\lambda_{\boldsymbol{g}}}$ is a resolution of $B / I_{\boldsymbol{g}}=B / I$. On the other hand, $\boldsymbol{R}_{\lambda_{\boldsymbol{g}}}$ is an $\boldsymbol{R}_{w}$-algebra; so,

$$
\mathbb{F} \otimes_{\boldsymbol{R}} \boldsymbol{R}_{\lambda_{\boldsymbol{g}}}=\mathbb{F} \otimes_{\boldsymbol{R}}\left(\boldsymbol{R}_{w} \otimes_{\boldsymbol{R}_{w}} \boldsymbol{R}_{\lambda_{\boldsymbol{g}}}\right)=\mathbb{F}_{w} \otimes_{\boldsymbol{R}_{w}} \boldsymbol{R}_{\lambda_{\boldsymbol{g}}}=\left(\left(\mathbb{F}_{\mathbb{Z}}\right)_{w_{\mathbb{Z}}} \otimes_{\mathbb{Z}} \boldsymbol{k}\right) \otimes_{\boldsymbol{R}_{w}} \boldsymbol{R}_{\lambda_{\boldsymbol{g}}}
$$

The definition of $\alpha:\left(\boldsymbol{R}_{\mathbb{Z}}\right)_{w_{\mathbb{Z}}} \rightarrow \boldsymbol{R}_{\lambda_{\boldsymbol{g}}}$ shows that

$$
\left(\left(\mathbb{F}_{\mathbb{Z}}\right)_{w_{\mathbb{Z}}} \otimes_{\mathbb{Z}} \boldsymbol{k}\right) \otimes_{\boldsymbol{R}_{w}} \boldsymbol{R}_{\lambda_{\boldsymbol{g}}}=\left(\mathbb{F}_{\mathbb{Z}}\right)_{w_{\mathbb{Z}}} \otimes_{\left(\boldsymbol{R}_{\mathbb{Z}}\right)_{w_{\mathbb{Z}}}} \boldsymbol{R}_{\lambda_{\boldsymbol{g}}}
$$

Thus, $\left(\mathbb{F}_{\mathbb{Z}}\right)_{w_{\mathbb{Z}}} \otimes_{\left(\boldsymbol{R}_{\mathbb{Z}}\right)_{w_{\mathbb{Z}}}} \boldsymbol{R}_{\lambda_{\boldsymbol{g}}}$ is a resolution of $B / I$ by free $B$-modules. The construction

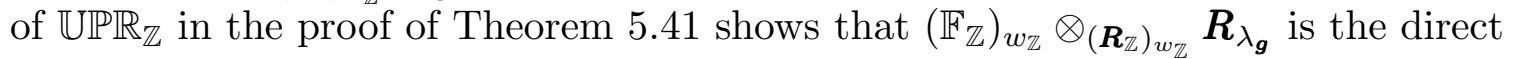
sum of the subcomplexes $\mathbb{U} \mathbb{R}_{\mathbb{Z}} \otimes_{\left(\boldsymbol{R}_{\mathbb{Z}}\right)_{w_{\mathbb{Z}}}} \boldsymbol{R}_{\lambda_{\boldsymbol{g}}}$ and $\mathbb{S}$, where $\mathbb{S}$ is the exact complex

$$
0 \rightarrow\left[\left(\boldsymbol{S}_{\mathbb{Z}}\right)_{w_{\mathbb{Z}}} \otimes_{\left(\boldsymbol{R}_{\mathbb{Z}}\right)_{w_{\mathbb{Z}}}} \boldsymbol{R}_{\lambda_{\boldsymbol{g}}}\right](-3 c) \rightarrow\left[\left(\boldsymbol{S}_{\mathbb{Z}}\right)_{w_{\mathbb{Z}}} \otimes_{\left(\boldsymbol{R}_{\mathbb{Z}}\right)_{w_{\mathbb{Z}}}} \boldsymbol{R}_{\lambda_{\boldsymbol{g}}}\right](-3 c) \rightarrow 0,
$$

concentrated in positions two and three. Keep in mind that

$$
\left(\boldsymbol{S}_{\mathbb{Z}}\right)_{w_{\mathbb{Z}}} \otimes_{\left(\boldsymbol{R}_{\mathbb{Z}}\right)_{w_{\mathbb{Z}}}} \boldsymbol{R}_{\lambda_{\boldsymbol{g}}}=\boldsymbol{R}_{\lambda_{\boldsymbol{g}}}[x, y] \cong \boldsymbol{k}[x, y]=B
$$

The Quillen-Suslin theorem guarantees that every finitely generated projective $B$ module is free. We conclude that $\mathbb{U P R _ { \mathbb { Z } }} \otimes_{\left(\boldsymbol{R}_{\mathbb{Z}}\right)_{w_{\mathbb{Z}}}} \boldsymbol{R}_{\lambda_{\boldsymbol{g}}}$ is a resolution of $B / I$ by free $B$-modules and the graded Betti numbers of $\mathbb{U} \mathbb{R}_{\mathbb{Z}} \otimes_{\left(\boldsymbol{R}_{\mathbb{Z}}\right)_{w_{\mathbb{Z}}}} \boldsymbol{R}_{\lambda_{\boldsymbol{g}}}$ are given in (5.47).

The conclusion in Theorem 5.41 that $\left(G_{1}, G_{2}, G_{3}\right)\left(\boldsymbol{S}_{\mathbb{Z}}\right)_{w_{\mathbb{Z}}}$ is a perfect ideal is particularly useful because of the "Persistence of Perfection Principle", which is also known as the "transfer of perfection" (see [19, Prop. 6.14] or [5, Thm. 3.5]).

The Persistence of Perfection Principle. Let $R \rightarrow S$ be a homomorphism of Noetherian rings, $I$ be a perfect ideal of $R$ of grade $g$, and $\mathbb{P}$ be a resolution of $R / I$ by projective $R$-modules. If $I S$ is a proper ideal of $S$ with grade at least $g$, then $I S$ is a perfect ideal of $S$ of grade $g$ and $\mathbb{P} \otimes_{R} S$ is a resolution of $S / I S$ by projective $S$-modules.

In the context of Definition 5.21, we have identified two projective resolutions for $\left(\Delta_{\mathbb{Z}}\right)_{w_{\mathbb{Z}}}$ : the length three free resolution $\left(\mathbb{F}_{\mathbb{Z}}\right)_{w_{\mathbb{Z}}}$ of part $(2)$ of Theorem 5.41 and the length two projective resolution $\mathbb{U P R}_{\mathbb{Z}}$ of $(5.44)$. In part (4) of Theorem 5.41 we have identified a free resolution of length two for $\left(\Delta_{\mathbb{Z}}\right)_{w_{\mathbb{Z}} z_{0, j}}$. The Persistence of Perfection Principle yields the following result. 
Corollary 5.48. Adopt the notation of Definition 5.21 and let $S$ be an arbitrary Noetherian ring.

(1) If $\left(\boldsymbol{S}_{\mathbb{Z}}\right)_{w_{\mathbb{Z}}} \rightarrow S$ is a ring homomorphism and $\left(G_{1}, G_{2}, G_{3}\right) S$ is a proper ideal of grade at least 2 , then $\left(G_{1}, G_{2}, G_{3}\right) S$ is a perfect ideal of grade equal to 2 and

$$
\left(\mathbb{F}_{\mathbb{Z}}\right)_{w_{\mathbb{Z}}} \otimes_{\left(\boldsymbol{S}_{\mathbb{Z}}\right)_{w_{\mathbb{Z}}}} S \quad \text { and } \quad \mathbb{U P} \mathbb{R}_{\mathbb{Z}} \otimes_{\left(\boldsymbol{S}_{\mathbb{Z}}\right)_{w_{\mathbb{Z}}}} S
$$

both are projective resolutions of $S /\left(G_{1}, G_{2}, G_{3}\right) S$.

(2) If $\left(\boldsymbol{S}_{\mathbb{Z}}\right)_{w_{\mathbb{Z}} z_{0, j}} \rightarrow S$ is a ring homomorphism and $\left(G_{1}, G_{2}, G_{3}\right) S$ is a proper ideal of grade at least 2 , then $\left(G_{1}, G_{2}, G_{3}\right) S$ is a perfect ideal of grade equal to 2 and $\mathbb{F}_{\mathbb{Z}}^{(j)} \otimes_{\left(\boldsymbol{S}_{\mathbb{Z}}\right)_{w_{\mathbb{Z}} z_{0}, j}} S$ is free resolution of $S /\left(G_{1}, G_{2}, G_{3}\right) S$ of length 2.

\section{Section 6. Decomposition of the space of true triples.}

Let $k$ be an algebraically closed field and $d=2 c$ be an even integer. Recall the open subsets $\mathbb{B}_{d} \subseteq \mathbb{T}_{d} \subseteq \mathbb{A}_{d}$ of triples of forms of degree $d$ from Definition 5.4. In Observation 5.7 we saw that $\boldsymbol{g} \mapsto \Psi_{\boldsymbol{g}}$ gives a surjection from $\mathbb{T}_{d}$ to the space of true (i.e., birational and base-point free) parameterizations of plane curves of degree $d$. In this section we decompose $\mathbb{T}_{d}$ into locally closed subsets which depend on the configuration of multiplicity $c$ singularities on, or infinitely near, the corresponding curve. Recall the Configuration Poset $(\mathrm{CP}, \leq)$ from Definition 4.2.

Definition 6.1. For each \# in CP define

$$
S_{\#}=\left\{\begin{array}{l|l}
\boldsymbol{g} \in \mathbb{T}_{d} & \begin{array}{l}
\text { the configuration of multiplicity } c \text { singularities on or infinitely } \\
\text { near } \mathcal{C}_{\boldsymbol{g}} \text { are described by \# }
\end{array}
\end{array}\right\}
$$

and define $T_{\#}=\bigcup_{\#^{\prime} \leq \#} S_{\#^{\prime}}$.

It is clear that $\mathbb{T}_{d}$ is the disjoint union

$$
\mathbb{T}_{d}=\bigcup_{\# \in \mathrm{CP}} S_{\#}=T_{\emptyset}
$$

It is shown in Corollary 2.5 that $S_{\#} \subseteq \mathbb{B}_{d}$ for all $\#$ in CP, except $\#=\emptyset$; thus

$$
S_{\emptyset}=\left(S_{\emptyset} \cap \mathbb{B}_{d}\right) \cup \mathbb{U B}_{d}
$$

where $\mathbb{U B}_{d}=\mathbb{T}_{d} \backslash \mathbb{B}_{d}$, as was defined in Remark 5.5. It follows that the space $\mathbb{T}_{d}$ is equal to the disjoint union of the eight subsets

$$
\bigcup_{\# \in \mathrm{CP} \backslash\{\emptyset\}} S_{\#} \cup\left(S_{\emptyset} \cap \mathbb{B}_{d}\right) \cup \mathbb{U B}_{d}
$$


The set $\mathbb{U B}_{d}$ is a closed subset of $\mathbb{T}_{d}$; indeed it is shown in Theorem 5.24 that $\mathbb{U B}_{d}$ is a hypersurface section of $\mathbb{T}_{d}$. Once $\mathbb{U B}_{d}$ is removed from $\mathbb{T}_{d}$, then one is left with the space $\mathbb{B}_{d}$ which is the disjoint union of the seven subsets $\bigcup_{\# \in \mathrm{CP} \backslash\{\emptyset\}} S_{\#} \cup\left(S_{\emptyset} \cap \mathbb{B}_{d}\right)$. We call each of these seven subsets of $\mathbb{B}_{d}$ a stratum of $\mathbb{B}_{d}$. We consider the picture:

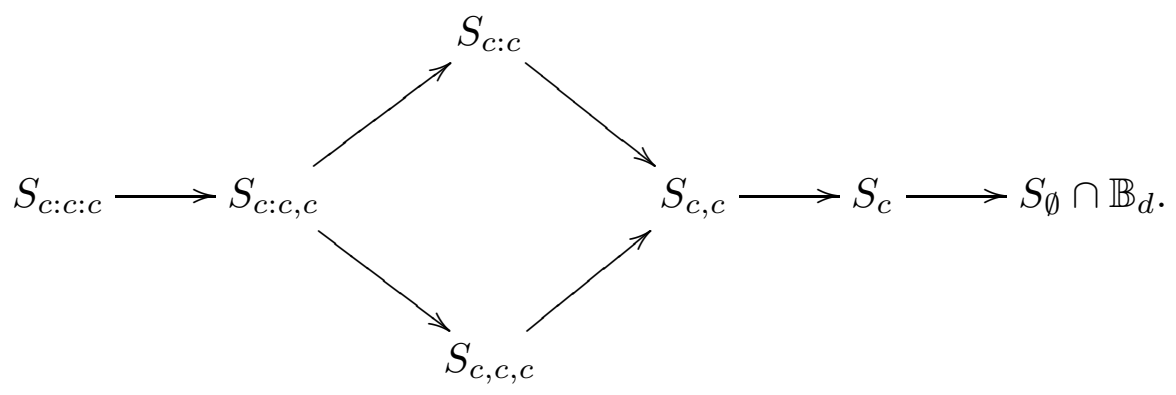

In this section, we prove that each stratum $S_{\#} \cap \mathbb{B}_{d}$ in the above picture is a locally closed subset of $\mathbb{B}_{d}$ and wherever we have drawn $S_{\#^{\prime}} \rightarrow S_{\#} \cap \mathbb{B}_{d}$, then $S_{\#^{\prime}}$ is contained in the closure $\overline{S_{\#} \cap \mathbb{B}_{d}}$ of $S_{\#} \cap \mathbb{B}_{d}$ in $\mathbb{B}_{d}$; indeed, we prove that $\overline{S_{\#} \cap \mathbb{B}_{d}}=T_{\#} \cap \mathbb{B}_{d}$. Furthermore, we prove that each $T_{\#} \cap \mathbb{B}_{d}$ is a closed irreducible subset of $\mathbb{B}_{d}$ and we calculate its dimension. We emphasize that if $\# \in \mathrm{CP}$, then

$$
\begin{cases}\#<\emptyset \Longrightarrow S_{\#}=S_{\#} \cap \mathbb{B}_{d} & \text { and } T_{\#}=T_{\#} \cap \mathbb{B}_{d}, \text { whereas } \\ \#=\emptyset \Longrightarrow S_{\emptyset}=\left(S_{\emptyset} \cap \mathbb{B}_{d}\right) \cup \mathbb{U B}_{d} & \text { and } T_{\emptyset}=\left(T_{\emptyset} \cap \mathbb{B}_{d}\right) \cup \mathbb{U B}_{d}=\mathbb{T}_{d}\end{cases}
$$

with $T_{\emptyset} \cap \mathbb{B}_{d}=\mathbb{B}_{d}$.

The set $\mathbb{B}_{d}$ is an open subset of the affine space $\mathbb{A}_{d}$ of Data 5.1 and the topology on $\mathbb{B}_{d}$ is the subspace topology: a subset of $Y$ of $\mathbb{B}_{d}$ is closed in $\mathbb{B}_{d}$ if and only $Y=\mathbb{B}_{d} \cap X$ for some closed subset $X$ of $\mathbb{A}_{d}$.

We now connect the subsets $S_{\#}$ and $T_{\#}$ of $\mathbb{T}_{d} \subseteq \mathbb{A}_{d}$ with the subsets $M_{\natural}$ and $N_{\#}$ of $\mathbb{B H}_{d} \subseteq \mathbb{H}_{d}$. Recall the morphisms

$$
G \times \mathbb{H}_{d} \stackrel{\Upsilon}{\rightarrow} \mathbb{H}_{d} \quad \text { and } \quad \mathbb{H}_{d} \stackrel{\Phi}{\rightarrow} \mathbb{A}_{d}
$$

from Remark (6) of 4.4 and Definition 5.28. The definitions of the sets $\mathbb{T}_{d}, \mathbb{B}_{d}$, and $\mathbb{B H}_{d}$ ensures

$$
\begin{gathered}
\Phi^{-1}\left(\mathbb{A}_{d} \backslash \mathbb{T}_{d}\right)=\mathbb{H}_{d} \backslash \mathbb{B H}_{d}, \\
\Phi^{-1}\left(\mathbb{T}_{d} \backslash \mathbb{B}_{d}\right) \text { is empty, and } \\
\Phi^{-1}\left(\mathbb{B}_{d}\right)=\mathbb{B H}_{d} .
\end{gathered}
$$

Furthermore, the restriction of $\Phi$ to $\mathbb{B H}_{d}$ is a surjection onto $\mathbb{B}_{d}$ :

$$
\Phi: \mathbb{B H}_{d} \rightarrow \mathbb{B}_{d} .
$$


In Definition 6.1, we decomposed $\mathbb{B}_{d}$ as $\cup_{\# \in \mathrm{CP}} S_{\#} \cap \mathbb{B}_{d}$ and in Theorem 4.9 we decomposed $\mathbb{B H}_{d}$ as $\cup_{\natural \in \mathrm{ECP}} \mathrm{DO}_{\natural}$. Furthermore,

$$
\Phi^{-1}\left(S_{\#} \cap \mathbb{B}_{d}\right)= \begin{cases}\mathrm{DO}_{\#} & \text { if } \#<c \\ \mathrm{DO}_{c, \mu_{4}} \cup \mathrm{DO}_{c, \mu_{5}} & \text { if } \#=c \\ \mathrm{DO}_{\emptyset, \mu_{4}} \cup \mathrm{DO}_{\emptyset, \mu_{5}} \cup \mathrm{DO}_{\emptyset, \mu_{6}} & \text { if } \#=\emptyset\end{cases}
$$

We have accounted for all eleven elements of ECP because, as was noted in Remark (3) of $4.11, \mathrm{DO}_{\mu_{2}}$ is empty.

Each orbit $\mathrm{DO}_{\natural}$ is defined (in Definition 4.7) to be $\mathrm{DO}_{\natural}=G \cdot M_{\natural}$. Recall from (4) of Definition 4.7 that

$$
M_{c}=M_{c, \mu_{4}} \cup M_{c, \mu_{5}} .
$$

Using this definition, we have

$$
\Phi^{-1}\left(S_{\#} \cap \mathbb{B}_{d}\right)=G \cdot M_{\#} \quad \text { for all } \# \in \mathrm{CP} \backslash\{\emptyset\}
$$

Fix $\# \in \mathrm{CP}$, with $\#<\emptyset$. Recall from (6.4) that $S_{\#} \cap \mathbb{B}_{d}=S_{\#}$. We have

$$
M_{\#} \subseteq G \cdot M_{\#}=\Phi^{-1}\left(S_{\#} \cap \mathbb{B}_{d}\right)=\Phi^{-1}\left(S_{\#}\right) \stackrel{\Phi}{\longrightarrow} S_{\#}
$$

Keep $\# \in \mathrm{CP}$, with $\#<\emptyset$. Apply $\Phi^{-1}$ to

$$
T_{\#}=\bigcup_{\substack{\#^{\prime} \in \mathrm{CP} \\ \#^{\prime} \leq \#}} S_{\#}
$$

to see that

$$
\Phi^{-1}\left(T_{\#}\right)=\bigcup_{\substack{\#^{\prime} \in \mathrm{CP} \\ \#^{\prime} \leq \#}} \Phi^{-1}\left(S_{\#}\right)=\bigcup_{\substack{\#^{\prime} \in \mathrm{CP} \\ \#^{\prime} \leq \#}} G \cdot M_{\#^{\prime}}=\bigcup_{\substack{\natural \in \mathrm{ECP} \\ \natural \leq \#}} \mathrm{DO}_{\natural}
$$

Definition 4.17 gives

$$
\bigcup_{\substack{\natural \in \mathrm{ECP} \\ \natural \leq \#}} \mathrm{DO}_{\natural}=\mathrm{CO}_{\#}
$$

and assertion (2) of Theorem 4.18 gives $\mathrm{CO}_{\#}=G \cdot N_{\#}$. Thus

$$
N_{\#} \subseteq G \cdot N_{\#}=\Phi^{-1}\left(T_{\#}\right) \stackrel{\Phi}{\longrightarrow} T_{\#}
$$


Combine (6.6) and (6.7) to obtain the following picture for all $\# \in \mathrm{CP}$ with $\#<\emptyset$.

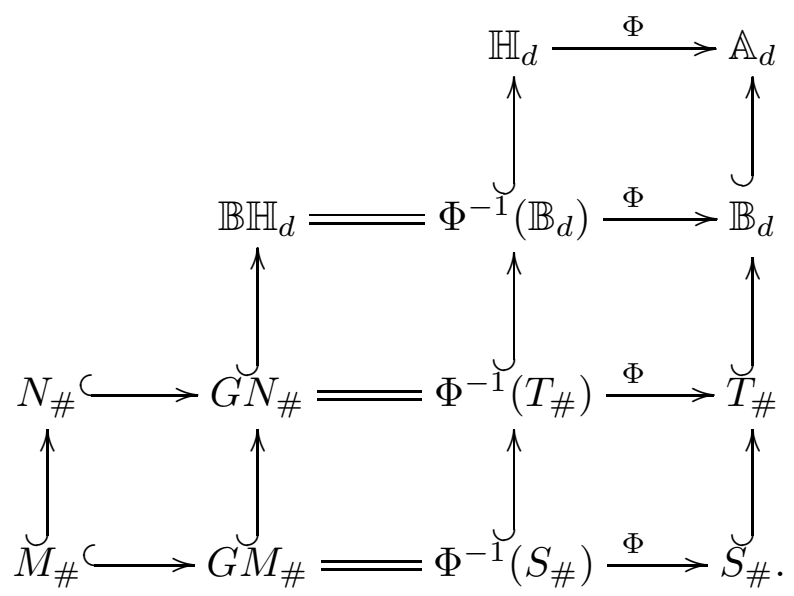

Remark 6.9. It follows from Proposition 4.21 that each stratum $S_{\#}$ is non-empty for $c \geq 3$; and if $c=2$, then $S_{\#}$ is non-empty if and only if $\# \leq\{c, c, c\}$.

Theorem 6.10. For every $\# \in \mathrm{CP}$, the set $T_{\#} \cap \mathbb{B}_{d}$ is a closed and irreducible subset of $\mathbb{B}_{d}$.

Proof. The statement is true, but not particularly interesting, for $\#=\emptyset$ because $T_{\emptyset}=\mathbb{T}_{d}$; and therefore $T_{\emptyset} \cap \mathbb{B}_{d}=\mathbb{B}_{d}$ which is a closed subset of $\mathbb{B}_{d}$. Furthermore, $\mathbb{B}_{d}$ is an open subset of the affine space $\mathbb{A}_{d}$; so $\mathbb{B}_{d}$ is irreducible.

Henceforth, we take $\# \neq \emptyset$. In this case, $T_{\#} \subseteq \mathbb{B}_{d}$; hence, $T_{\#} \cap \mathbb{B}_{d}=T_{\#}$. We first prove that $T_{\#}$ is a closed subset of $\mathbb{B}_{d}$. Recall the open cover $\cup_{i=1}^{3} \mathbb{B}_{d}^{(i)}$ of $\mathbb{B}_{d}$ which is given in the Remark after Theorem 5.30. It suffices to prove that $T_{\#} \cap \mathbb{B}_{d}^{(i)}$ is a closed subset of $\mathbb{B}_{d}^{(i)}$ for each $i$. Adopt the notation of Conventions 5.15 ; in particular, $\boldsymbol{R}=\boldsymbol{k}[\boldsymbol{z}]$ is the coordinate ring of $\mathbb{A}^{3 d+3}$. Let $A^{(i)}$ be the matrix of Remark 5.33 with entries from $\boldsymbol{R}[\boldsymbol{u}]$, and let $S_{i}$ and $D_{i}$ be the $\boldsymbol{R}$-algebras $S_{i}=\boldsymbol{R}[\boldsymbol{u}] / I_{3}\left(A^{(i)}\right)$ and $D_{i}=S_{i} / \operatorname{Jac}\left(S_{i} / \boldsymbol{R}\right)$. Corollary 3.23 shows that if $\boldsymbol{g} \in \mathbb{B}_{d}^{(i)}$, then the configuration of multiplicity $c$ singularities on or infinitely near $\mathcal{C}_{\boldsymbol{g}}$ may be read from the rings $\left(S_{i}\right)_{\lambda_{\boldsymbol{g}}}$ and $\left(D_{i}\right)_{\lambda_{\boldsymbol{g}}}$. We proved in Corollary 3.23 that

$$
\begin{aligned}
T_{c} & \cap \mathbb{B}_{d}^{(i)}=X\left(S_{i} ; \geq 1\right) \cap \mathbb{B}_{d}^{(i)} \\
T_{c, c} \cap \mathbb{B}_{d}^{(i)} & =X\left(S_{i} ;=1, \geq 2\right) \cap \mathbb{B}_{d}^{(i)} \\
T_{c: c} \cap \mathbb{B}_{d}^{(i)} & =X\left(S_{i} ; \geq 1\right) \cap X\left(D_{i}, \geq 1\right) \cap \mathbb{B}_{d}^{(i)}=X\left(D_{i}, \geq 1\right) \cap \mathbb{B}_{d}^{(i)} \\
T_{c, c, c} \cap \mathbb{B}_{d}^{(i)} & =X\left(S_{i} ;=1, \geq 3\right) \cap \mathbb{B}_{d}^{(i)} \\
T_{c: c, c} & \cap \mathbb{B}_{d}^{(i)}=X\left(S_{i} ;=1, \geq 3\right) \cap X\left(D_{i} ; \geq 1\right) \cap \mathbb{B}_{d}^{(i)}
\end{aligned}
$$




$$
T_{c: c: c} \cap \mathbb{B}_{d}^{(i)}=X\left(S_{i} ;=1, \geq 3\right) \cap X\left(D_{i} ;=1, \geq 2\right) \cap \mathbb{B}_{d}^{(i)}=X\left(D_{i} ;=1, \geq 2\right) \cap \mathbb{B}_{d}^{(i)}
$$

Apply Theorem 5.13, by way of Convention (4) of 5.15. The set $X\left(S_{i} ; \geq 1\right) \cap \mathbb{A}_{d}$ is closed in $\mathbb{A}_{d}$; hence, $T_{c} \cap \mathbb{B}_{d}^{(i)}$ is a closed subset of $\mathbb{B}_{d}^{(i)}$. The set $X\left(S_{i} ;=1, \geq 2\right) \cap \mathbb{B}_{d}^{(i)}$ is closed in

$$
X\left(S_{i},=1\right) \cap \mathbb{B}_{d}^{(i)}=X\left(S_{i} ; \geq 1\right) \cap \mathbb{B}_{d}^{(i)} ;
$$

but $X\left(S_{i} ; \geq 1\right) \cap \mathbb{B}_{d}^{(i)}$ is already closed in $\mathbb{B}_{d}^{(i)}$, so, $T_{c, c} \cap \mathbb{B}_{d}^{(i)}$ is closed in $\mathbb{B}_{d}^{(i)}$. The set $X\left(D_{i} ; \geq 1\right) \cap \mathbb{B}_{d}^{(i)}$ is closed in $\mathbb{B}_{d}^{(i)}$; so, $T_{c: c} \cap \mathbb{B}_{d}^{(i)}$ is closed in $\mathbb{B}_{d}^{(i)}$. The set $X\left(S_{i} ;=1, \geq 3\right) \cap \mathbb{B}_{d}^{(i)}$ is closed in $X\left(S_{i} ;=1\right) \cap \mathbb{B}_{d}^{(i)}$. The argument from $T_{c, c}$ yields that $T_{c, c, c} \cap \mathbb{B}_{d}^{(i)}$ is closed in $\mathbb{B}_{d}^{(i)}$. The set $T_{c: c, c} \cap \mathbb{B}_{d}^{(i)}$ is the intersection of the closed subsets $T_{c, c, c} \cap \mathbb{B}_{d}^{(i)}$ and $T_{c: c} \cap \mathbb{B}_{d}^{(i)}$; so, $T_{c: c, c} \cap \mathbb{B}_{d}^{(i)}$ is also a closed subset of $\mathbb{B}_{d}^{(i)}$. The set $X\left(D_{i} ;=1, \geq 2\right) \cap \mathbb{B}_{d}^{(i)}$ is closed in the closed set $X\left(D_{i} ; \geq 1\right) \cap \mathbb{B}_{d}^{(i)}$; so, $T_{c: c: c} \cap \mathbb{B}_{d}^{(i)}$ is a closed set in $\mathbb{B}_{d}^{(i)}$.

Fix $\# \in \mathrm{CP}$ with $\# \neq \emptyset$. We have established that each set $T_{\#}$ is a closed subset of $\mathbb{B}_{d}$. Now we show that $T_{\#}$ is irreducible. Consider the surjective morphisms

$$
G \times N_{\#} \stackrel{\Upsilon}{\longrightarrow} \Phi^{-1}\left(T_{\#}\right) \stackrel{\Phi}{\longrightarrow} T_{\#} .
$$

We have shown in Theorem 4.18 that $N_{\#}$ is an irreducible variety. It is clear that $G$ is an irreducible variety; hence, $G \times N_{\#}$ is irreducible. It follows that $T_{\#}$ and $\Phi^{-1}\left(T_{\#}\right)$ both are also irreducible.

Corollary 6.12. If $\# \in \mathrm{CP}$ and, either $c \geq 3$; or else, $c=2$ and $\# \leq\{c, c, c\}$, then

(1) $S_{\#}=T_{\#} \backslash\left(\bigcup_{\#^{\prime}<\#} T_{\#^{\prime}}\right)$,

(2) $S_{\emptyset} \cap \mathbb{B}_{d}$ is open in $\mathbb{T}_{d}$ and $S_{\emptyset}=\left(S_{\emptyset} \cap \mathbb{B}_{d}\right) \cup \mathbb{U B}_{d}$ is the union of an open subset of $\mathbb{T}_{d}$ and a closed subset of $\mathbb{T}_{d}$,

(3) $S_{\#} \cap \mathbb{B}_{d}$ is open in $T_{\#} \cap \mathbb{B}_{d}$ and is locally closed in $\mathbb{T}_{d}$,

(4) $T_{\#} \cap \mathbb{B}_{d}$ is the closure of $S_{\#} \cap \mathbb{B}_{d}$ in $\mathbb{B}_{d}$

(5) $\mathbb{T}_{d}$ is the closure of $S_{\emptyset}$ in $\mathbb{T}_{d}$,

(6) $S_{c: c: c}=T_{c: c: c}$ is closed in $\mathbb{B}_{d}$, and

(7) $S_{\#} \cap \mathbb{B}_{d}$ is irreducible.

Proof. Assertion (1) follows from the definition of $T_{\#}$. We next prove (2). The subset $T_{c}$ of $\mathbb{B}_{d}$ is closed by Theorem 6.10 ; so, $S_{\emptyset} \cap \mathbb{B}_{d}$, which is equal to $\mathbb{B}_{d} \backslash T_{c}$, is open in $\mathbb{B}_{d}$. But, $\mathbb{B}_{d}$ is open in $\mathbb{T}_{d}$; so $S_{\emptyset} \cap \mathbb{B}_{d}$ is also open in $\mathbb{T}_{d}$. The second part of (2) is established in (6.2) and Theorem 5.24. The first assertion of (3) follows from (1) and the fact that $\mathbb{T}_{\#} \cap \mathbb{B}_{d}$ is closed in $\mathbb{B}_{d}$. The second assertion of (3) also 
uses the fact that $\mathbb{B}_{d}$ is open in $\mathbb{T}_{d}$. We prove (4). The subset $S_{\#} \cap \mathbb{B}_{d}$ of $\mathbb{T}_{\#} \cap \mathbb{B}_{d}$ is open by (3) and non-empty by Remark 6.9. Theorem 6.10 shows that $\mathbb{T}_{\#} \cap \mathbb{B}_{d}$ is irreducible. For $(5)$, use the fact that $\mathbb{T}_{d}$ is irreducible and that $S_{\emptyset}$ contains a non-empty open subset $S_{\emptyset} \cap \mathbb{B}_{d}$ of $\mathbb{T}_{d}$; see (2). Assertion (6) is clear and (7) follows from (4) and the fact that $T_{\#} \cap \mathbb{B}_{d}$ is irreducible.

Theorem 6.13. Assume $c \geq 3$. The picture (6.3) gives a stratification of $\mathbb{B}_{d}$. In other words, $\mathbb{B}_{d}$ is the disjoint union of $\left\{S_{\#} \cap \mathbb{B}_{d} \mid \# \in \mathrm{CP}\right\}$ and if $\#^{\prime} \leq \#$ in $\mathrm{CP}$, then $S_{\#^{\prime}} \cap \mathbb{B}_{d}$ is contained in the closure $\overline{S_{\#} \cap \mathbb{B}_{d}}$ of $S_{\#} \cap \mathbb{B}_{d}$ in $\mathbb{B}_{d}$.

Proof. The fact that $\mathbb{B}_{d}$ is the disjoint union of $\left\{S_{\#} \cap \mathbb{B}_{d} \mid \# \in \mathrm{CP}\right\}$ follows from the geometric description of $S_{\#}$ : any given curve has exactly one configuration of multiplicity $c$ singularities. The poset $\mathrm{CP}$ contains all possible configurations as was discussed at the beginning of Section 4. The second assertion follows from Corollary 6.12 (4) and the definition of $T_{\#}$.

Theorem 6.14. Assume $c \geq 3$. For each fixed $\# \in \mathrm{CP}$, the sets $T_{\#}$ and $S_{\#}$ have the same dimension and this dimension is given in the following chart:

$$
\begin{array}{cccccccc}
\# & c: c: c & c: c, c & c, c, c & c: c & c, c & c & \emptyset \\
\operatorname{dim} S_{\#} & 3 c+7 & 3 c+8 & 3 c+9 & 4 c+6 & 4 c+7 & 5 c+5 & 6 c+3
\end{array}
$$

Proof. The closure of $S_{\emptyset}$ in $\mathbb{T}_{d}$ is $T_{\emptyset}=\mathbb{T}_{d}$. It follows that

$$
\operatorname{dim} S_{\emptyset}=\operatorname{dim} T_{\emptyset}=\mathbb{T}_{d}
$$

On the other hand, $\mathbb{T}_{d}$ is a non-empty open subset of $\mathbb{A}_{d}$; so, its dimension is $6 c+3$.

Henceforth, we consider $\# \in \mathrm{CP}$ with $\# \neq \emptyset$. We have seen that $T_{\#}$ is irreducible and that $S_{\#}$ is a non-empty open subset of $T_{\#}$. Hence, $\operatorname{dim} T_{\#}=\operatorname{dim} S_{\#}$. We now compute this dimension. Recall from (6.11) that $G \times N_{\#}, T_{\#}$, and $\Phi^{-1}\left(T_{\#}\right)$ all are irreducible.

Notice that for every $\boldsymbol{g} \in T_{\#}$ with $\boldsymbol{g}=\Phi(\varphi)$, we have

$$
\Phi^{-1}(\boldsymbol{g})=\left(\left\{I_{3 \times 3}\right\} \times \mathrm{SL}_{2}(\boldsymbol{k})\right) \varphi \cong \mathrm{SL}_{2}(\boldsymbol{k}),
$$

which has dimension 3 . Thus, since $T_{\#}$ is irreducible, the surjective morphism on the right hand side of (6.11) yields that

$$
\operatorname{dim} T_{\#}=\operatorname{dim} \Phi^{-1}\left(T_{\#}\right)-3
$$

Indeed, once we pass to the closure of $T_{\#}$ and $\Phi^{-1}\left(T_{\#}\right)$, in their respective affine spaces, then the map $\Phi$ corresponds to an embedding of affine $k$-domains and the 
given formula follows from the additivity of transcendence degrees; see, for example, [11, Cor 14.6]. We apply the same technique to the surjective morphism on the left hand side of (6.11) to see that

$$
\operatorname{dim} \Phi^{-1}\left(T_{\#}\right)=\operatorname{dim}\left(G \times N_{\#}\right)-\operatorname{dim} \Upsilon^{-1}(\varphi)
$$

for general $\varphi$. Combine (6.15) and (6.16) to see

$$
\operatorname{dim} T_{\#}=\operatorname{dim} \Phi^{-1}\left(T_{\#}\right)-3=\operatorname{dim}\left(G \times N_{\#}\right)-\operatorname{dim} \Upsilon^{-1}(\varphi)-3
$$

and therefore,

$$
\operatorname{dim} T_{\#}=\operatorname{dim} N_{\#}-\operatorname{dim} \Upsilon^{-1}(\varphi)+10
$$

We compute the dimensions of the fibers $\Upsilon^{-1}(\varphi)$, for $\varphi$ general in $\Phi^{-1}\left(T_{\#}\right)$.

Observe that $\Phi^{-1}\left(S_{\#}\right)$ is a dense open subset of $\Phi^{-1}\left(T_{\#}\right)$. Indeed, the set $\Phi^{-1}\left(T_{\#}\right)$ is irreducible, the subset $S_{\#}$ of $T_{\#}$ is open, and the morphism $\Phi$ is continuous. Thus, we may assume that the general element $\varphi$ of $\Phi^{-1}\left(T_{\#}\right)$ is actually in $\varphi \in \Phi^{-1}\left(S_{\#}\right)$ and we can write $\varphi=h \varphi_{M}$ for some $h \in G$ and $\varphi_{M} \in M_{\#}$, see (6.8). Now

$$
\begin{aligned}
\Upsilon^{-1}(\varphi) & =\left\{(g, \psi) \in G \times N_{\#} \mid g \psi=\varphi\right\} \\
& =\left\{(g, \psi) \in G \times N_{\#} \mid g \psi=h \varphi_{M}\right\} \\
& =\left\{(g, \psi) \in G \times N_{\#} \mid \psi=g^{-1} h \varphi_{M}\right\} \\
& =\left\{\left(g, g^{-1} h \varphi_{M}\right) \in G \times N_{\#}\right\} \\
& \cong\left\{g \in G \mid g^{-1} h \varphi_{M} \in N_{\#}\right\} \\
& =\left\{h k \in G \mid k^{-1} \varphi_{M} \in N_{\#}\right\} \\
& \cong\left\{k \in G \mid k^{-1} \varphi_{M} \in N_{\#}\right\}
\end{aligned}
$$

Define

$$
F_{\#}\left(\varphi_{M}\right)=\left\{k \in G \mid k^{-1} \varphi_{M} \in N_{\#}\right\} .
$$

We compute the dimension of $F_{\#}\left(\varphi_{M}\right)$ for various choices of \#.

First let $\#=c$. Recall that

$$
N_{c}=\left\{\left(\begin{array}{cc}
Q_{1} & Q_{2} \\
Q_{3} & Q_{4} \\
0 & Q_{5}
\end{array}\right)\right\} \cap \Phi^{-1}\left(\mathbb{B}_{d}\right)
$$


We show that

$$
F_{c}\left(\varphi_{M}\right)=\left\{\left(\left[\begin{array}{ccc}
x_{1} & x_{2} & x_{3} \\
x_{4} & x_{5} & x_{6} \\
0 & 0 & x_{7}
\end{array}\right],\left(\begin{array}{cc}
y_{1} & y_{2} \\
0 & y_{3}
\end{array}\right)\right) \in G\right\}
$$

which has dimension 10. Once (6.18) is established, then we use $\operatorname{dim} N_{c}=5 c+5$ and (6.17) to see that $\operatorname{dim} T_{c}=5 c+5-10+10=5 c+5$.

It is clear that the inclusion $(\supseteq)$ holds in (6.18). To show the other inclusion, let $k \in F_{c}\left(\varphi_{M}\right)$. Now $\varphi_{M}$, as well as $k^{-1} \varphi_{M}$ are in $N_{c}$. As $\varphi$ is general in $\Phi^{-1}\left(T_{c}\right)$ and this set contains matrices with five linearly independent entries, it follows that $\mu\left(I_{1}(\varphi)\right) \geq 5$; therefore, $\mu\left(I_{1}\left(\varphi_{M}\right)\right) \geq 5$ and $\mu\left(I_{1}\left(k^{-1} \varphi_{M}\right)\right) \geq 5$. Thus,

$$
\varphi_{M}=\left(\begin{array}{cc}
Q_{1} & Q_{2} \\
Q_{3} & Q_{4} \\
0 & Q_{5}
\end{array}\right) \quad \text { and } \quad k^{-1} \varphi_{M}=\left(\begin{array}{cc}
Q_{1}^{\prime} & Q_{2}^{\prime} \\
Q_{3}^{\prime} & Q_{4}^{\prime} \\
0 & Q_{5}^{\prime}
\end{array}\right)
$$

where $\left\{Q_{1}, \ldots, Q_{5}\right\}$ and $\left\{Q_{1}^{\prime}, \ldots, Q_{5}^{\prime}\right\}$ are linearly independent sets.

Let $\mathcal{C}_{1}$ and $\mathcal{C}_{2}$ be the curves parameterized by $\Phi\left(\varphi_{M}\right)$ and $\Phi\left(k^{-1} \varphi_{M}\right)$, respectively. Then $\mathcal{C}_{1}$ and $\mathcal{C}_{2}$ have exactly one singularity of multiplicity $c$, and this singularity is the point $[0: 0: 1]$. (See Theorem 4.8). Write $k=\left(\chi^{-1}, \xi\right) \in$ $\mathrm{GL}_{3}(\boldsymbol{k}) \times \mathrm{GL}_{2}(\boldsymbol{k})$. Notice that $\chi$ induces a linear automorphism of $\mathbb{P}^{2}$ that maps $\mathcal{C}_{1}$ to $\mathcal{C}_{2}$ (Remark 0.9 ) and hence leaves the point $[0: 0: 1]$ fixed. It follows that the third row of $\chi$ is $[0,0, a]$ for some $a \in k^{*}$. Since

$$
\left(\chi \varphi_{M} \xi\right)_{(3,1)}=\left(k^{-1} \varphi_{M}\right)_{(3,1)}=0
$$

we obtain $[0,0, a] \varphi_{M} \xi_{1}=0$ when $\xi_{1}$ is the first column of $\xi$. Clearly,

$$
[0,0, a] \varphi_{M}\left[\begin{array}{l}
1 \\
0
\end{array}\right]=0
$$

Hence Remark 3.17 gives $\xi_{1}=\left[\begin{array}{l}b \\ 0\end{array}\right]$, for some $b \in k^{*}$. It follows that $k=\left(\chi^{-1}, \xi\right)$ has the form of the elements in the right hand side of (6.18).

For $\#=c, c$, recall that

$$
N_{c, c}=\left\{\left[\begin{array}{cc}
Q_{1} & Q_{2} \\
Q_{3} & Q_{4} \\
0 & Q_{5}
\end{array}\right] \mid \operatorname{dim}\left(Q_{3}, Q_{4}, Q_{5}\right) \leq 2\right\} \cap \Phi^{-1}\left(\mathbb{B}_{d}\right)
$$


which has dimension $4 c+4$. In this case, we have

$$
F_{c, c}\left(\varphi_{M}\right)=\left\{k \in G \mid k^{-1} \varphi_{M} \in N_{c, c}\right\}
$$

We now show that

$$
F_{c, c}\left(\varphi_{M}\right)=\left\{k \in G \mid k^{-1} \varphi_{M} \in M_{c, c}\right\}
$$

Indeed, $\varphi_{M} \in M_{c, c} \subseteq \Phi^{-1}\left(S_{c, c}\right)$. Thus, $k^{-1} \varphi_{M} \in \Phi^{-1}\left(S_{c, c}\right)$. Hence, if $k^{-1} \varphi_{M} \in$ $N_{c, c}$, then

$$
k^{-1} \varphi_{M} \in N_{c, c} \cap \Phi^{-1}\left(S_{c, c}\right)=M_{c, c} .
$$

Recall that

$$
M_{c, c}=\left\{\left[\begin{array}{cc}
Q_{1} & Q_{2} \\
Q_{3} & Q_{3} \\
0 & Q_{4}
\end{array}\right] \mid \operatorname{dim}_{k}<Q_{1}, Q_{2}, Q_{3}, Q_{4}>=4\right\} \cap \Phi^{-1}\left(\mathbb{B}_{d}\right) .
$$

We now claim that

$$
F_{c, c}\left(\varphi_{M}\right)=\left\{\left(\left[\begin{array}{ccc}
x_{1} & x_{2} & x_{3} \\
0 & x_{4} & 0 \\
0 & 0 & x_{5}
\end{array}\right],\left[\begin{array}{cc}
y_{1} & y_{2} \\
0 & y_{1}-y_{2}
\end{array}\right]\right)\right\} \cup\left\{\left(\left[\begin{array}{ccc}
x_{1} & x_{2} & x_{3} \\
0 & 0 & x_{4} \\
0 & x_{5} & 0
\end{array}\right],\left[\begin{array}{cc}
y_{1} & y_{2} \\
-y_{1} & -y_{1}
\end{array}\right]\right)\right\}
$$

which has dimension 7 . Thus, this claim, together with (6.17) gives $\operatorname{dim} T_{c, c}=$ $4 c+7$. Clearly, the left hand side of (6.19) contains the right hand side. To show the other containment, let $k=\left(\chi^{-1}, \xi\right)$ be an element of $F_{c, c}\left(\varphi_{M}\right)$. As before, let $\mathcal{C}_{1}$ and $\mathcal{C}_{2}$ be the curves parameterized by $\Phi\left(\varphi_{M}\right)$ and $\Phi\left(k^{-1} \varphi_{M}\right)$. Then $\mathcal{C}_{1}$ and $\mathcal{C}_{2}$ have exactly two singularities of multiplicity $c$ : one at the point $[0: 1: 0]$ and the other at $[0: 0: 1]$. Since $\chi$ induces a linear automorphism of $\mathbb{P}^{2}$ mapping $\mathcal{C}_{1}$ to $\mathcal{C}_{2}$ and hence a permutation of the points $[0: 1: 0]$ and $[0: 0: 1]$, we have that $\chi$ is of the form

$$
\left[\begin{array}{ccc}
x_{1} & x_{2} & x_{3} \\
0 & x_{4} & 0 \\
0 & 0 & x_{5}
\end{array}\right] \quad \text { or } \quad\left[\begin{array}{ccc}
x_{1} & x_{2} & x_{3} \\
0 & 0 & x_{4} \\
0 & x_{5} & 0
\end{array}\right] \text {. }
$$

As noted before, the BiProj Lemma (Remark 3.17) now determines $\xi$ in both cases. This establishes the claim.

For $\#=c: c$, recall that

$$
N_{c: c}=\left\{\left[\begin{array}{cc}
Q_{1} & Q_{2} \\
Q_{3} & Q_{4} \\
0 & Q_{3}
\end{array}\right]\right\} \cap \Phi^{-1}\left(\mathbb{B}_{d}\right)
$$

which has dimension $4 c+4$. As before, we have

$$
F_{c: c}\left(\varphi_{M}\right)=\left\{k \in G \mid k^{-1} \varphi_{M} \in N_{c: c}\right\}=\left\{k \in G \mid k^{-1} \varphi_{M} \in M_{c: c}\right\} .
$$


Now recall that

$$
M_{c: c}=\left\{\left[\begin{array}{cc}
Q_{1} & Q_{2} \\
Q_{3} & Q_{4} \\
0 & Q_{3}
\end{array}\right] \mid \operatorname{dim}_{\boldsymbol{k}}<Q_{1}, Q_{2}, Q_{3}, Q_{4}>=4\right\} \cap \Phi^{-1}\left(\mathbb{B}_{d}\right) .
$$

We now claim that

$$
F_{c: c}\left(\varphi_{M}\right)=\left\{\left(\left[\begin{array}{ccc}
x_{1} & x_{2} & x_{3} \\
0 & x_{4} & x_{5} \\
0 & 0 & x_{6}
\end{array}\right]^{-1},\left[\begin{array}{cc}
y_{1} & y_{2} \\
0 & y_{3}
\end{array}\right]\right) \mid x_{4} y_{1}=x_{6} y_{3}\right\}
$$

which has dimension 8. Thus, this claim, together with $(6.17)$, gives $\operatorname{dim} T_{c: c}=$ $4 c+6$.

One easily sees that the left hand side of (6.20) contains the right hand side. To show the other containment, let $k=\left(\chi^{-1}, \xi\right)$ be in $F_{c: c}\left(\varphi_{M}\right)$. As before, one proves that the last row of $\chi$ is of the form $[0,0, a]$ and the first column of $\xi$ is of the form $\xi_{1}=\left[\begin{array}{l}b \\ 0\end{array}\right]$. Therefore, we can write

$$
\chi=\left[\begin{array}{ccc}
a_{1} & a_{2} & a_{3} \\
a_{4} & a_{5} & a_{6} \\
0 & 0 & a_{7}
\end{array}\right] \quad \text { and } \quad \xi=\left[\begin{array}{cc}
b_{1} & b_{2} \\
0 & b_{3}
\end{array}\right]
$$

Writing $\varphi_{M}=\left[\begin{array}{cc}Q_{1} & Q_{2} \\ Q_{3} & Q_{4} \\ 0 & Q_{3}\end{array}\right]$ one obtains

$$
\left[\chi \varphi_{M} \xi\right]_{(2,1)}=a_{4} b_{1} Q_{1}+a_{5} b_{1} Q_{3} \quad \text { and } \quad\left[\chi \varphi_{M} \xi\right]_{(3,2)}=a_{7} b_{3} Q_{3}
$$

Since $\chi \varphi_{M} \xi=k^{-1} \varphi_{M} \in M_{c: c}$, we conclude that $a_{4} b_{1}=0$ and $a_{5} b_{1}=a_{7} b_{3}$. As $\xi \in \mathrm{GL}_{2}(\boldsymbol{k})$, one has $b_{1} \neq 0$; therefore, $a_{4}=0$ and the claim now follows.

For $\#=c, c, c$, recall that

$$
N_{c, c, c}=\left\{\left[\begin{array}{cc}
Q_{1} & Q_{2} \\
Q_{3} & Q_{4} \\
0 & Q_{5}
\end{array}\right] \mid \operatorname{dim}_{k}<Q_{1}, Q_{2}, Q_{3}, Q_{4}, Q_{5}>\leq 3\right\} \cap \Phi^{-1}\left(\mathbb{B}_{d}\right),
$$

which has dimension $3 c+3$. As before,

$$
F_{c, c, c}\left(\varphi_{M}\right)=\left\{k \in G \mid k^{-1} \varphi_{M} \in N_{c, c, c}\right\}=\left\{k \in G \mid k^{-1} \varphi_{M} \in M_{c, c, c}\right\}
$$

Now,

$$
M_{c, c, c}=\left\{\left[\begin{array}{cc}
Q_{1} & Q_{1} \\
Q_{2} & 0 \\
0 & Q_{3}
\end{array}\right] \mid \operatorname{dim}_{k}<Q_{1}, Q_{2}, Q_{3}>=3\right\} \cap \Phi^{-1}\left(\mathbb{B}_{d}\right)
$$


thus, we claim that $F_{c, c, c}\left(\varphi_{M}\right)$ is equal to

$$
\begin{aligned}
& \left\{\left(\left[\begin{array}{ccc}
x_{1} & 0 & 0 \\
0 & x_{2} & 0 \\
0 & 0 & x_{3}
\end{array}\right],\left[\begin{array}{ll}
y & 0 \\
0 & y
\end{array}\right]\right) \in G\right\} \quad \cup\left\{\left(\left[\begin{array}{ccc}
0 & 0 & x_{1} \\
0 & x_{2} & 0 \\
x_{3} & 0 & 0
\end{array}\right],\left[\begin{array}{cc}
-y & 0 \\
y & y
\end{array}\right]\right) \in G\right\} \\
& \cup\left\{\left(\left[\begin{array}{ccc}
0 & x_{1} & 0 \\
x_{2} & 0 & 0 \\
0 & 0 & x_{3}
\end{array}\right],\left[\begin{array}{cc}
y & y \\
0 & -y
\end{array}\right]\right) \in G\right\} \cup\left\{\left(\left[\begin{array}{ccc}
0 & 0 & x_{1} \\
x_{2} & 0 & 0 \\
0 & x_{3} & 0
\end{array}\right]^{-1},\left[\begin{array}{cc}
0 & -y \\
y & y
\end{array}\right]\right) \in G\right\} \\
& \cup\left\{\left(\left[\begin{array}{ccc}
0 & x_{1} & 0 \\
0 & 0 & x_{2} \\
x_{3} & 0 & 0
\end{array}\right]^{-1},\left[\begin{array}{cc}
y & y \\
-y & 0
\end{array}\right]\right) \in G\right\} \cup\left\{\left(\left[\begin{array}{ccc}
x_{1} & 0 & 0 \\
0 & 0 & x_{2} \\
0 & x_{3} & 0
\end{array}\right],\left[\begin{array}{ll}
0 & y \\
y & 0
\end{array}\right]\right) \in G\right\},
\end{aligned}
$$

which has dimension 4 . Thus, $\operatorname{dim} T_{c, c, c}=3 c+9$. It is easy to see that subset of $G$ listed in (6.21) is contained in $F_{c, c, c}\left(\varphi_{M}\right)$. To see the reverse inclusion, observe that the curves $\mathcal{C}_{1}$ and $\mathcal{C}_{2}$, parameterized by $\Phi\left(\varphi_{M}\right)$ and $\Phi\left(k^{-1} \varphi_{M}\right)$ have exactly three singularities of multiplicity $c$ and they are at points $[1: 0: 0],[0: 1: 0]$, and $[0: 0: 1]$. Thus, if $k=\left(\chi^{-1}, \xi\right)$ is in $F_{c, c, c}\left(\varphi_{M}\right)$, then the linear automorphism of $\mathbb{P}^{2}$ induced by $\chi$ gives a permutation of these 3 points. Hence, $\chi$ is of the form $\left[\begin{array}{ccc}x_{1} & 0 & 0 \\ 0 & x_{2} & 0 \\ 0 & 0 & x_{3}\end{array}\right] \sigma$, where $\sigma$ is one of the six permutation matrices. Once again the BiProj Lemma determines the shape of $\xi$, and the claim follows.

If $\#=c: c: c$, recall that

$$
N_{c: c: c}=\left\{\left[\begin{array}{cc}
Q_{1} & Q_{2} \\
Q_{3} & Q_{1} \\
0 & Q_{3}
\end{array}\right] \mid \operatorname{dim}_{\boldsymbol{k}}<Q_{1}, Q_{2}, Q_{3}>=3\right\} \cap \Phi^{-1}\left(\mathbb{B}_{d}\right)=M_{c: c: c}
$$

which has dimension $3 c+3$. We claim that

$$
F_{c: c: c}\left(\varphi_{M}\right)=\left\{\left(\left[\begin{array}{ccc}
x_{1} & x_{2} & x_{3} \\
0 & x_{4} & x_{5} \\
0 & 0 & x_{6}
\end{array}\right]^{-1},\left[\begin{array}{cc}
y_{1} & y_{2} \\
0 & y_{3}
\end{array}\right]\right) \in G \mid \begin{array}{c}
x_{6} y_{3}=x_{4} y_{1} \\
x_{5} y_{3}=x_{2} y_{1}-x_{4} y_{2} \\
x_{4} y_{3}=x_{1} y_{1}
\end{array}\right\}
$$

Since $y_{3} \neq 0$, the dimension of the fiber is 6 ; thus, $\operatorname{dim} T_{c: c: c}=3 c+7$. A matrix calculation gives immediately the containment of the right hand side in the left hand side of (6.22). To prove the other containment, one sees as before, that for $k=\left(\chi^{-1}, \xi\right)$ in $F_{c: c: c}\left(\varphi_{M}\right)$, one has $\chi=\left[\begin{array}{ccc}a_{1} & a_{2} & a_{3} \\ a_{4} & a_{5} & a_{6} \\ 0 & 0 & a_{7}\end{array}\right]$ and $\xi=\left[\begin{array}{cc}b_{1} & b_{2} \\ 0 & b_{3}\end{array}\right]$. Writing $\varphi_{M}=\left[\begin{array}{cc}Q_{1} & Q_{2} \\ Q_{3} & Q_{1} \\ 0 & Q_{3}\end{array}\right]$, we obtain

$$
\chi \varphi_{M} \xi=\left[\begin{array}{cc}
a_{1} b_{1} Q_{1}+a_{2} b_{1} Q_{3} & * \\
a_{4} b_{1} Q_{1}+a_{5} b_{1} Q_{3} & \left(a_{4} b_{2}+a_{5} b_{3}\right) Q_{1}+a_{4} b_{3} Q_{2}+\left(a_{5} b_{2}+a_{6} b_{3}\right) Q_{3} \\
0 & a_{7} b_{3} Q_{3}
\end{array}\right] .
$$


Since this matrix is in $M_{c: c: c}$, we conclude that $a_{4} b_{1}$ and $a_{4} b_{3}$ are both zero, which gives $a_{4}=0$ since neither $b_{1}$ nor $b_{3}$ can be zero. Using this, we also obtain

$$
a_{2} b_{1}=a_{5} b_{2}+a_{6} b_{3}, \quad a_{1} b_{1}=a_{5} b_{3} \quad \text { and } \quad a_{5} b_{1}=a_{7} b_{3}
$$

which are the required conditions.

Finally, to compute the dimension of $T_{c: c, c}$, notice that one has proper containments of closed irreducible subsets of $\mathbb{B}_{d}$ :

$$
T_{c: c: c} \subsetneq T_{c: c, c} \subsetneq T_{c, c, c},
$$

where $\operatorname{dim} T_{c: c: c}=3 c+7$ while $\operatorname{dim} T_{c, c, c}=3 c+9$. It follows that $\operatorname{dim} T_{c: c, c}=$ $3 c+8$.

\section{SECTION 7. The JACOBIAN MATRIX AND THE RAMIFICATION LOCUS.}

Remark 1.31 provides a method of parameterizing the branches of a parameterized curve. Theorem 7.2 shows that the Jacobian matrix associated to the parameterization identifies the non-smooth branches of the curve as well as the multiplicity of each branch. The starting point for this line of reasoning is the result that if $D$ is an algebra which is essentially of finite type over the $\operatorname{ring} C$, then the ramification locus of $D$ over $C$ is equal to the support of the module of Kähler differentials $\Omega_{D / C}$. (See, for example, [22, Cor. 6.10].) In our ultimate application of this result, we consider the module of differentials $\Omega$ for the ring extension

$$
\frac{\hat{R}}{J_{i}} \rightarrow \hat{S}_{\mathfrak{M}_{i}}
$$

from the proof of Lemma 1.7. We have two presentations of the $\hat{S}_{\mathfrak{M}_{i}}$-module $\Omega$. One presentation comes from the Jacobian matrix of the parameterization of the curve $\mathcal{C}$. The other presentation comes from the geometry which gives rise to the ring extension (7.1). The Fitting ideal Fitt $_{0}(\Omega)$ may be computed using either presentation.

In addition to [22] one may consult [11, Chapt. 16] or [27, Sect. 26] for elementary facts and notation pertaining to Kähler differentials.

Theorem 7.2. Adopt the Data of 1.1 with $\boldsymbol{k}$ an algebraically closed field of characteristic zero. Consider the inclusion map $k\left[I_{d}\right] \subseteq B$ of homogeneous coordinate rings which is induced by the morphism $\Psi: \mathbb{P}^{1} \rightarrow \mathcal{C}$. The gcd of the zeroth Fitting ideal of $\Omega_{B / \boldsymbol{k}\left[I_{d}\right]}$ is a polynomial in $B$. Let

$$
\operatorname{gcdFitt}_{0}\left(\Omega_{B / \boldsymbol{k}\left[I_{d}\right]}\right)=\prod_{i=1}^{s} \ell_{i}^{f_{i}},
$$


where $\left(\ell_{1}\right), \ldots,\left(\ell_{s}\right)$ are distinct height one linear ideals of $B$. If $(\ell)$ is an arbitrary height one linear ideal of $B$ and $\mathcal{C}(\ell)$ is the branch of $\mathcal{C}$ which corresponds to $\ell$, in the sense of Remark 1.31, then the multiplicity of the branch $\mathcal{C}(\ell)$ is

$$
\begin{cases}f_{i}+1 & \text { if }(\ell)=\left(\ell_{i}\right) \text { for some } i \\ 1 & \text { otherwise. }\end{cases}
$$

Furthermore, the Fitting ideal Fitt $_{0}\left(\Omega_{B / \boldsymbol{k}\left[I_{d}\right]}\right)$ is equal to the ideal $I_{2}(N)$ of $B$, where $N$ is the $2 \times n$ transposed Jacobian matrix

$$
N=\left[\begin{array}{lll}
\frac{\partial g_{1}}{\partial x} & \cdots & \frac{\partial g_{n}}{\partial x} \\
\frac{\partial g_{1}}{\partial y} & \cdots & \frac{\partial g_{n}}{\partial y}
\end{array}\right]
$$

Before proving Theorem 7.2, we describe various special cases. Corollary 7.3 follows immediately from Theorem 7.2 without any further proof. Also, Theorem 7.4 requires only a small amount of additional proof.

Corollary 7.3. Retain the notation and hypotheses of Theorem 7.2.

(1) All of the branches through all of the points of $\mathcal{C}$ are smooth if and only if ht $I_{2}(N)=2$.

(2) The multiplicity of each branch of $\mathcal{C}$ is at most two if and only if the gcd of $I_{2}(N)$ decomposes into a product of pairwise non-associated linear factors.

Theorem 7.4. Adopt Data 1.1 with $\boldsymbol{k}$ an algebraically closed field of characteristic zero. Let $p_{1}, \ldots, p_{z}$ be the singularities of $\mathcal{C}$. For each singular point $p_{j}$, let $m_{j}$ be the multiplicity of $\mathcal{C}$ at $p_{j}$ and $s_{j}$ be the number of branches of $\mathcal{C}$ at $p_{j}$. For each index $j$, with $1 \leq j \leq z$, let $\operatorname{gcd} I_{1}\left(p_{j} \varphi\right)=\prod_{u=1}^{s_{j}} \ell_{u, j}^{e_{u, j}}$, where the $\ell_{u, j}$ are pairwise non-associated linear factors and the exponents $e_{u, j}$ are positive. Let

$$
N=\left[\begin{array}{lll}
\frac{\partial g_{1}}{\partial x} & \cdots & \frac{\partial g_{n}}{\partial x} \\
\frac{\partial g_{1}}{\partial y} & \cdots & \frac{\partial g_{n}}{\partial y}
\end{array}\right]
$$

be the $2 \times n$ Jacobian matrix of the parametrization. Then

(1) $\operatorname{gcd} I_{2}(N)=\prod_{j=1}^{z} \prod_{u=1}^{s_{j}} \ell_{u, j}^{e_{u, j}-1}$, and

(2) the degree of $\operatorname{gcd} I_{2}(N)$ is equal to $\sum_{j=1}^{z}\left(m_{j}-s_{j}\right)$. 
Proof. We are given the singular points $p_{1}, \ldots, p_{z}$ on $\mathcal{C}$ and the factorizations $\operatorname{gcd} I_{1}\left(p_{j} \varphi\right)=\prod \ell_{u, j}^{e_{u, j}}$. Lemma 1.7 tells us that the multiplicity of the branch $\mathcal{C}\left(\ell_{u, j}\right)$ of $\mathcal{C}$ is $e_{u, j}$. Assertion (1) is now an immediate consequence Theorem 7.2. We prove (2). Theorem 1.8 shows that for each $j$, with $1 \leq j \leq z$, the polynomial $\operatorname{gcd} I_{1}\left(p_{j} \varphi\right)$ has degree $m_{j}$ and $s_{j}$ pairwise non-associated linear factors; so, one has

$$
\operatorname{deg} \prod_{u=1}^{s_{j}} \ell_{u, j}^{e_{u, j}-1}=\operatorname{deg} \prod_{u=1}^{s_{j}} \ell_{u, j}^{e_{u, j}}-s_{j}=m_{j}-s_{j}
$$

hence, (1) gives

$$
\operatorname{deg} \operatorname{gcd} I_{2}(N)=\operatorname{deg} \prod_{j=1}^{z} \prod_{u=1}^{s_{j}} \ell_{u, j}^{e_{u, j}-1}=\sum_{j=1}^{z}\left(m_{j}-s_{j}\right)
$$

Proof of Theorem 7.2. The relative cotangent complex

$$
\Omega_{\boldsymbol{k}\left[I_{d}\right] / \boldsymbol{k}} \otimes_{\boldsymbol{k}} B \rightarrow \Omega_{B / \boldsymbol{k}} \rightarrow \Omega_{B / \boldsymbol{k}\left[I_{d}\right]} \rightarrow 0
$$

gives rise to the presentation

$$
B^{n} \stackrel{N}{\rightarrow} B^{2} \rightarrow \Omega_{B / \boldsymbol{k}\left[I_{d}\right]} \rightarrow 0
$$

of $\Omega_{B / \boldsymbol{k}\left[I_{d}\right]}$ as a $B$-module. It follows immediately that the Fitting ideal of the $B$-module $\Omega_{B / \boldsymbol{k}\left[I_{d}\right]}$ is

$$
\operatorname{Fitt}_{0}\left(\Omega_{B / \boldsymbol{k}\left[I_{d}\right]}\right)=I_{2}(N) B \text {. }
$$

Fix a point $q \in \mathbb{P}^{1}$ and a non-zero linear form $\ell \in B$ with $\ell(q)=0$. Let $p$ be the point $\left(g_{1}(q), \ldots, g_{n}(q)\right)$ on $\mathcal{C}$ in $\mathbb{P}^{n-1}$ and $e$ be the multiplicity of the branch $\mathcal{C}(\ell)$. Define $f$ to be the exponent with

$$
\operatorname{gcd} I_{2}(N)=\ell^{f} \cdot \theta
$$

where $\theta$ is a polynomial in $B$ which is relatively prime to $\ell$. We prove $e=f+1$. Let $\mathfrak{p}$ be the ideal

$$
I_{2}\left(\begin{array}{ccc}
g_{1}(q) & \ldots & g_{n}(q) \\
g_{1} & \ldots & g_{n}
\end{array}\right)
$$

of $\boldsymbol{k}\left[I_{d}\right]$.

The formation of $\Omega$ is preserved under base change. That is, if $C^{\prime}$ and $D$ are $C$-algebras, then

$$
C^{\prime} \otimes_{C} \Omega_{D / C}=\Omega_{\left(C^{\prime} \otimes_{C} D\right) / C^{\prime}}
$$


In particular, any presentation of $\Omega_{D / C}$ as an $D$-module:

$$
D^{a} \stackrel{\sigma}{\rightarrow} D^{b} \stackrel{\tau}{\rightarrow} \Omega_{D / C} \rightarrow 0
$$

gives rise to a presentation of $\Omega_{\left(C^{\prime} \otimes_{C} D\right) / C^{\prime}}$ as an $C^{\prime} \otimes_{C} D$-module:

$$
\left(C^{\prime} \otimes_{C} D\right)^{a} \stackrel{\sigma}{\rightarrow}\left(C^{\prime} \otimes_{C} D\right)^{b} \stackrel{\tau}{\rightarrow} C^{\prime} \otimes_{C} \Omega_{D / C}=\Omega_{\left(C^{\prime} \otimes_{C} D\right) / C^{\prime}} \rightarrow 0 .
$$

For example, if $C^{\prime}=U^{-1} C$ for some multiplicatively closed subset $U$ of $C$, then we write $U^{-1} D$ in place of $U^{-1} C \otimes_{C} D$; so we have the presentation

$$
\left(U^{-1} D\right)^{a} \stackrel{\sigma}{\rightarrow}\left(U^{-1} D\right)^{b} \stackrel{\tau}{\rightarrow} \Omega_{U^{-1} D / U^{-1} C} \rightarrow 0
$$

In our situation, we localize at $U=\boldsymbol{k}\left[I_{d}\right] \backslash \mathfrak{p}$. We write $\boldsymbol{k}\left[I_{d}\right]_{\mathfrak{p}}$ in place of $U^{-1} \boldsymbol{k}\left[I_{d}\right]$ and $B_{\mathfrak{p}}$ in place of $U^{-1}(B)$. Apply the base change $\boldsymbol{k}\left[I_{d}\right]_{\mathfrak{p}} \otimes_{\boldsymbol{k}\left[I_{d}\right]}$ to (7.5) to obtain the following presentation by free $B_{\mathfrak{p}}$-modules

$$
B_{\mathfrak{p}}^{n} \stackrel{N}{\rightarrow} B_{\mathfrak{p}}^{2} \rightarrow \Omega_{B_{\mathfrak{p}} / \boldsymbol{k}\left[I_{d}\right]_{\mathfrak{p}}} \rightarrow 0 .
$$

In the statement of Lemma 1.7 we have called $\boldsymbol{k}\left[I_{d}\right]_{\mathfrak{p}}=R \subseteq S=B_{\mathfrak{p}}$. The ring $R$ is local with maximal ideal $\mathfrak{m}_{R}=\mathfrak{p} R_{\mathfrak{p}}$. In this new language, (7.7) becomes the exact sequence of $S$-modules:

$$
S^{n} \stackrel{N}{\rightarrow} S^{2} \rightarrow \Omega_{S / R} \rightarrow 0
$$

Complete both $R \subseteq S$ in the $\mathfrak{m}_{R}$-adic topology to obtain the rings $\hat{R} \subseteq \hat{S}$. One of the maximal ideals of $\hat{S}$ is $(\ell) \hat{S}$, which we denote by $\mathfrak{M}$. Let $J$ be the kernel of $\hat{R} \rightarrow \hat{S}_{\mathfrak{M}}$. It is shown at $(1.23)$ that the multiplicity $e(\hat{R} / J)$ is equal to $e$, which is the multiplicity of the branch $\mathcal{C}(\ell)$.

Apply the base change $\hat{R} \otimes_{R}$ to (7.8) to obtain the exact sequence of $\hat{R} \otimes_{R} S=\hat{S}$ modules:

$$
\hat{S}^{n} \stackrel{N}{\rightarrow} \hat{S}^{2} \rightarrow \Omega_{\hat{S} / \hat{R}} \rightarrow 0 .
$$

Localize at the multiplicatively closed set $\hat{S} \backslash \mathfrak{M}$ of $\hat{S}$ to obtain an exact sequence of $\hat{S}_{\mathfrak{M}}$-modules:

$$
\hat{S}_{\mathfrak{M}}^{n} \stackrel{N}{\rightarrow} \hat{S}_{\mathfrak{M}}^{2} \rightarrow \Omega_{\hat{S}_{\mathfrak{M} / \hat{R}}} \rightarrow 0
$$

Apply the base change $\hat{R} / J \otimes_{\hat{R}}$. Keep in mind that $\hat{R} / J \otimes_{\hat{R}} \hat{S}_{\mathfrak{M}}=\hat{S}_{\mathfrak{M}}$. Obtain an exact sequence of $\hat{S}_{\mathfrak{M}}$-modules

$$
\hat{S}_{\mathfrak{M}}^{n} \stackrel{N}{\rightarrow} \hat{S}_{\mathfrak{M}}^{2} \rightarrow \Omega_{\hat{S}_{\mathfrak{M} / \frac{\hat{R}}{J}}} \rightarrow 0
$$


The zeroth Fitting ideal of $\Omega_{\hat{S}_{\mathfrak{M} / \frac{\hat{R}}{J}}}$ is

$$
\operatorname{Fitt}_{0}\left(\Omega_{\hat{S}_{\mathfrak{M} / \frac{\hat{R}}{J}}}\right)=I_{2}(N) \hat{S}_{\mathfrak{M}}=\left(\ell^{f}\right) \hat{S}_{\mathfrak{M}}=\mathfrak{M}^{f} \hat{S}_{\mathfrak{M}}
$$

Now we calculate $\Omega_{\hat{S}_{\mathfrak{M}} / \frac{\hat{R}}{J}}$ in a completely different manner. Recall the Veronese ring $\boldsymbol{k}\left[B_{d}\right]$ and the ring $T=\boldsymbol{k}\left[B_{d}\right]_{\mathfrak{p}}$ from Lemma 1.7. The completion of $T$ in the $\mathfrak{m}_{R}$-adic topology is denoted $\hat{T}$. We have

$$
\hat{R} / J \subseteq \hat{T}_{\mathfrak{M} \cap \hat{T}} \subseteq \hat{S}_{\mathfrak{M}}
$$

with $\hat{T}_{\mathfrak{M} \cap \hat{T}}$ equal to the integral closure of $\hat{R} / J$. The rings $\hat{T}_{\mathfrak{M} \cap \hat{T}}$ and $\hat{R} / J$ share the same residue class field, which, in the language of the proof of Lemma 1.7, was called $\boldsymbol{k}\left(g_{n}^{\prime}\right)$. Furthermore, $\boldsymbol{k}\left(g_{n}^{\prime}\right) \subseteq \hat{R} / J$. The rings $\hat{T}_{\mathfrak{M} \cap \hat{T}}$ and $\hat{S}_{\mathfrak{M}}$ are complete DVRs with the same uniformizing parameter $t=\frac{\ell}{m}$, where $m$ is any linear form in $B$ for which $\ell, m$ is a basis for the vector space $B_{1}$. The ring $\hat{T}_{\mathfrak{M} \cap \hat{T}}$ is equal to $\boldsymbol{k}\left(m^{d}\right)[[t]]$ and the ring $\hat{S}_{\mathfrak{M}}$ is equal to $\boldsymbol{k}(m)[[t]]$. It was observed above that $e(\hat{R} / J)=e$. Proposition 7.11 shows that $\Omega_{\hat{S}_{\mathfrak{M} / \frac{\hat{R}}{J}}}$ is isomorphic to $\hat{S}_{\mathfrak{M}} /\left(t^{e-1}\right)$. We conclude that the Fitting ideal of $\Omega_{\hat{S}_{\mathfrak{M} / \frac{\hat{R}}{J}}}$ is also equal to

$$
\operatorname{Fitt}_{0}\left(\Omega_{\hat{S}_{\mathfrak{M} / \frac{\hat{R}}{J}}}\right)=\left(t^{e-1}\right) \hat{S}_{\mathfrak{M}}=\mathfrak{M}^{e-1} \hat{S}_{\mathfrak{M}}
$$

Compare (7.9) and (7.10) to see that $f=e-1$.

Proposition 7.11. Let $K \subseteq A \subseteq B \subseteq C$ be local rings. Assume that

(1) $B=L[[t]]$ and $C=M[[t]]$ are formal power series rings in one variable where $L \subseteq M$ are fields of characteristic zero with $\operatorname{dim}_{L} M$ finite,

(2) $K$ is a field and the natural maps

$$
K \rightarrow A \rightarrow A / \mathfrak{m}_{A} \quad \text { and } \quad K \rightarrow B \rightarrow B /(t)=L
$$

are isomorphisms,

(3) B finitely generated as an $A$-module, and

(4) $B \subseteq \operatorname{Quot}(A)$.

Then the $C$-modules $\Omega_{C / A}$ and $\frac{C}{\left(t^{-1}\right) C}$ are isomorphic, where $e=e(A)$ is the multiplicity of the local ring $A$.

Proof. The relative cotangent sequence gives an exact sequence of $C$-modules:

$$
\Omega_{A / K} \otimes_{A} C \stackrel{\alpha}{\rightarrow} \Omega_{C / K} \stackrel{\beta}{\rightarrow} \Omega_{C / A} \rightarrow 0
$$


where $\alpha(d a \otimes c)=(d a) c$ and $\beta(d c)=d c$. The $\operatorname{ring} C$ is generated as an $A$-algebra by $t$ together with a finite generating set for $M$ over $K$; so, $\Omega_{C / A}$ is finitely generated as a $C$-module. The ring $C$ is local; so the Krull Intersection Theorem guarantees that $\beta$ sends $\cap\left(t^{n}\right) \Omega_{C / K}$ to zero. Thus, the above exact sequence induces the exact sequence of $C$-modules

$$
\Omega_{A / K} \otimes_{A} C \stackrel{\bar{\alpha}}{\rightarrow} \bar{\Omega}_{C / K} \stackrel{\bar{\beta}}{\rightarrow} \Omega_{C / A} \rightarrow 0
$$

where

$$
\bar{\Omega}_{C / K}=\frac{\Omega_{C / K}}{\cap\left(t^{n}\right) \Omega_{C / K}}
$$

and $\bar{\alpha}$ and $\bar{\beta}$ are induced by $\alpha$ and $\beta$. If $\omega \in \Omega_{C / K}$, then we write $\bar{\omega}$ for the image of $\omega$ in $\bar{\Omega}_{C / K}$. The field extension $K \subseteq M$ is separable and algebraic so the universal derivation $d=d_{C / K}: C \rightarrow \Omega_{C / K}$ sends $M$ to zero. Therefore, if $f \in C$, then the elements $d f$ and $f^{\prime} d t$ of $\Omega_{C / K}$ represent the same class in $\bar{\Omega}_{C / K}$. It follows that $\bar{\Omega}_{C / K}$ is generated as a $C$-module by $\overline{d t}$. To complete the proof we show that

(1) $\bar{\Omega}_{C / K}$ is a free $C$-module and

(2) the image of $\bar{\alpha}$ is equal to $\left(t^{e-1}\right) C \overline{d t}$.

We prove (2) first. The one-dimensional domains $A \subseteq B$ are local and $B$ is the integral closure of $A$; so, Lemma 1.10 guarantees the existence of an element $z$ in $\mathfrak{m}_{A}$ such that $z=t^{e}+$ higher order terms and $\mathfrak{m}_{A} B=z B$. It follows that $\mathfrak{m}_{A} C=z C$. The image of $\bar{\alpha}$ is the $C$-submodule of $\bar{\Omega}_{C / K}=C \overline{d t}$ which is generated by $\bar{\alpha}(d z)$ and this is the equal to $\left(t^{e-1}\right) C \overline{d t}$ since the field $K$ has characteristic zero.

Now we prove (1). Suppose that $\theta \in C$ and that the element $\theta d t$ of $\Omega_{C / K}$ is in $\bigcap_{i=0}^{\infty}\left(t^{i}\right) \Omega_{C / K}$. We prove that $\theta$ is zero in $C$. Let $n$ be a positive integer. Consider the conormal exact sequence

$$
\left(t^{n}\right) /\left(t^{2 n}\right) \rightarrow \Omega_{C / K} \otimes_{C} C /\left(t^{n}\right) \rightarrow \Omega_{\frac{C}{\left(t^{n}\right)} / K} \rightarrow 0
$$

of $C /\left(t^{n}\right)$-modules associated to the $K$-algebra homomorphism $C \rightarrow C /\left(t^{n}\right)$. The sequence (7.12) induces an isomorphism

$$
\frac{\Omega_{C / K}}{\left(t^{n-1}\right) C d t+\left(t^{n}\right) C \Omega_{C / K}} \stackrel{\cong}{\rightrightarrows} \Omega_{\frac{C}{\left(t^{n}\right)} / K},
$$

which is given by class of $d f \mapsto d($ class of $f$ ), for all $f \in C$. The element $\theta d t$ of $\Omega_{C / K}$ represents the class of zero in the module on the left side of (7.13); so 
$\bar{\theta} d t$ is zero in $\Omega_{\frac{C}{\left(t^{n}\right)} / K}$, where $\bar{\theta}$ is the image of $\theta$ in $C /\left(t^{n}\right)$. On the other hand, $C /\left(t^{n}\right)=M[t] /\left(t^{n}\right)$ and it is well-known that $1 \mapsto d t$ gives an isomorphism

$$
\frac{M[t]}{\left(t^{n-1}\right)} \stackrel{\cong}{\longrightarrow} \Omega_{\frac{M[t]}{\left(t^{n}\right)} / K}
$$

Thus, the image $\bar{\theta}$ of $\theta$ in $C /\left(t^{n-1}\right)$ is zero. This process may be repeated for all $n$. We conclude that $\theta$ is zero in $C$.

\section{SECTION 8. The CONDUCTOR AND THE}

\section{BRANCHES OF A RATIONAL PLANE CURVE.}

Let $\boldsymbol{g}$ be an element of $\mathbb{T}_{d}$ and $\mathcal{C}_{\boldsymbol{g}}$ be the corresponding parameterized plane curve. We produce a homogeneous polynomial $c_{\boldsymbol{g}}$ in $B=\boldsymbol{k}[x, y]$. The linear factors of $c_{\boldsymbol{g}}$ correspond to the branches of $\mathcal{C}_{\boldsymbol{g}}$ at its singularities. (Recall from Remark 1.31 that the given parameterization of $\mathcal{C}_{\boldsymbol{g}}$ induces a one-to-one correspondence between the set of height one linear ideals of $B$ and the branches of $\mathcal{C}_{\boldsymbol{g}}$.) Moreover, the exponents that appear on the linear factors of $c_{\boldsymbol{g}}$ give information about the singularity degree $\delta_{p}$ at the corresponding singularity. See Theorem 8.11 for the general statement and Theorem 8.12 for the form of $c_{\boldsymbol{g}}$ for each of the 13 possible configurations of singularities on a quartic. We are able to capture some information about $\delta_{p}$ by way of $c_{\boldsymbol{g}}$ in a polynomial manner from the coefficients of the entries of a Hilbert-Burch matrix for $\boldsymbol{g}$. If $d$ is even and $\boldsymbol{g} \in \mathbb{B}_{d}$, then a Hilbert-Burch matrix for $\boldsymbol{g}$ may be built in a polynomial manner from the coefficients of $\boldsymbol{g}$, see Theorem 5.30. Therefore, we can use features of the factorization of $c_{\boldsymbol{g}}$ to separate, by way of open and closed subsets of $\mathbb{B}_{d}$, various configurations of singularities; see Theorem 8.18 and Proposition 8.20.

There are 13 possible configurations of singularities on a rational plane quartic, see (0.3). The techniques of Sections 1, 2, and 3 (which involve the multiplicity $m_{p}$ at each singular point $p$ ) and Section 7 (which involve the number of branches $s_{p}$ at each singular point $p$ ) are able to separate, using open and closed sets in $\mathbb{T}_{4}$, the 13 possible configurations of singularities into 12 subsets. The techniques of the previous sections are not able to separate those curves whose singularities are described by $(2: 2: 1),(2: 1,1)$ from those curves whose singularities are described by $(2: 2: 1,1),(2: 1)$. (This notation is explained at (0.5).) We were motivated to prove Theorem 8.18 and Proposition 8.20 in order to separate these two configurations of singularities.

The polynomial $c_{\boldsymbol{g}}$ is defined in Theorem 8.9. Let $\boldsymbol{c}_{\boldsymbol{g}}$ be the conductor of the $d^{\text {th }}$ Veronese subring $\boldsymbol{V}$ of $B=\boldsymbol{k}[x, y]$ into the coordinate ring $A_{\boldsymbol{g}}=\boldsymbol{k}\left[g_{1}, g_{2}, g_{3}\right]$ of $\mathcal{C}_{\boldsymbol{g}}$. In Corollary 8.4 we show that $V / \mathfrak{c}_{\boldsymbol{g}}$ is Cohen-Macaulay and we calculate its multiplicity. The polynomial $c_{\boldsymbol{g}}$ is the gcd of the extension $\mathfrak{c}_{\boldsymbol{g}} \cdot B$ of the ideal $\mathfrak{c}_{\boldsymbol{g}}$ in 
$\boldsymbol{V}$ to the larger ring $B$. The extension of $\mathfrak{c}_{\boldsymbol{g}}$ to $B$ does not define a Cohen-Macaulay quotient; however, the ease of computation in $B$ (as compared to $\boldsymbol{V}$ ) compensates for the inconvenience of having to saturate $\mathfrak{c}_{\boldsymbol{g}}$ in order to produce $c_{\boldsymbol{g}}$. Corollary 8.8 expresses $\mathfrak{c}_{\boldsymbol{g}}$ in terms of the Hilbert-Burch matrix for $\boldsymbol{g}$. Theorem 8.11 explains the geometric significance of $c_{\boldsymbol{g}}$.

Laurent Busé has shown us that polynomials similar to $c_{\boldsymbol{g}}$ have been studied elsewhere in the literature. Abhyankar [1, Pg. 153] called his version of this polynomial a Taylor resultant. Busé further developed the concept of Taylor resultants in paragraph 4.4 of [7]. The Taylor resultant is essentially the determinant of a square matrix over a Principal Ideal Domain. Recently Busé and D'Andrea [9] examined all of the invariant factors of the square matrix, not only the determinant, in order to gain much more detailed information about the blow-up history of the corresponding singularity. Even more recently, Busé and Luu Ba [8, Sect. 5] have applied these ideas to the study of singularities on rational space curves. In this context the matrix in question is no longer square; so, they use Fitting ideals in place of invariant factors.

Data 8.1. In this section $k$ continues to be a field, $B$ continues to be the polynomial ring $B=\boldsymbol{k}[x, y]$, and $d \geq 3$ is a fixed integer. Consider an ordered triple $\boldsymbol{g}=$ $\left(g_{1}, g_{2}, g_{3}\right)$ in the set $\mathbb{T}_{d}$ of Definition 5.4. Let $A_{\boldsymbol{g}}$ be the subring $\boldsymbol{k}\left[g_{1}, g_{2}, g_{3}\right]$ of $B$ and $T$ be the polynomial ring $\boldsymbol{k}\left[T_{1}, T_{2}, T_{3}\right]$. The ring $A_{\boldsymbol{g}}$ is a $T$-module by way of the $k$-algebra homomorphism $T \rightarrow A_{\boldsymbol{g}}$ which sends $T_{i}$ to $g_{i}$. Indeed, $A_{\boldsymbol{g}} \cong T /\left(f_{\boldsymbol{g}}\right)$ as described in (5.19). Let $V$ be the $d^{\text {th }}$ Veronese subring of $B$; so $V$ is the standard graded $k$-algebra $\boldsymbol{V}=\bigoplus_{i} \boldsymbol{V}_{i}$, with $\boldsymbol{V}_{i}=B_{i d}$. We have $A_{\boldsymbol{g}} \subseteq \boldsymbol{V} \subseteq B$. The assumption that $\boldsymbol{g}$ is in $\mathbb{T}_{d}$ ensures that $\left(g_{1}, g_{2}, g_{3}\right) B$ is an ideal in $B$ of height 2 and that $A_{\boldsymbol{g}} \subseteq \boldsymbol{V}$ is a birational extension. Define the conductor $\mathfrak{c}_{\boldsymbol{g}}$ by $\mathfrak{c}_{\boldsymbol{g}}=A_{\boldsymbol{g}}:_{Q} \boldsymbol{V}$, where $Q=\operatorname{Quot}\left(A_{\boldsymbol{g}}\right)$. Notice that $\mathfrak{c}_{\boldsymbol{g}}$ is an ideal of $V$ and also is an ideal of $A_{\boldsymbol{g}}$. Let $L_{\boldsymbol{g}}$ be the preimage of $\mathfrak{c}_{\boldsymbol{g}}$ in $T$. It follows that $T / L_{\boldsymbol{g}} \cong A_{\boldsymbol{g}} / \mathfrak{c}_{\boldsymbol{g}}$.

Lemma 8.2. Adopt Data 8.1. The homogeneous minimal free resolution of $T / L_{\boldsymbol{g}} \cong$ $A_{\boldsymbol{g}} / \mathfrak{c}_{\boldsymbol{g}}$ by free $T$-modules has the form

$$
0 \rightarrow T^{d-2}(-d+1) \rightarrow T^{d-1}(-d+2) \rightarrow T \rightarrow T / L_{\boldsymbol{g}} \rightarrow 0
$$

Furthermore, $T / L_{\boldsymbol{g}}$ is Cohen-Macaulay and $\omega_{T / L_{\boldsymbol{g}}} \cong \omega_{A_{\boldsymbol{g}} / \mathfrak{c}_{\boldsymbol{g}}} \cong \boldsymbol{V} / A_{\boldsymbol{g}}$.

Proof. The $a$-invariant of $B$ is -2 ; hence, $a(\boldsymbol{V})=\left\lfloor\frac{-2}{d}\right\rfloor=-1$. Therefore,

$$
\operatorname{reg}(\boldsymbol{V})=-1+\operatorname{dim} \boldsymbol{V}=1
$$

because $\boldsymbol{V}$ is Cohen-Macaulay. 
Since $\boldsymbol{V}$ is a finitely generated $T$-module, we have

$$
\operatorname{reg}_{T}(\boldsymbol{V})=\operatorname{reg}(\boldsymbol{V})=1
$$

Hence, $\boldsymbol{V}$ is generated in degree at most 1 as a $T$-module. Therefore, $\boldsymbol{V} / A_{\boldsymbol{g}}$ is generated in degree at most 1 as a $T$-module. Since $\left[\boldsymbol{V} / A_{\boldsymbol{g}}\right]_{0}=0$, it follows that $\boldsymbol{V} / A_{\boldsymbol{g}}$ is generated as a $T$-module in degree 1 . But $\left[\boldsymbol{V} / A_{\boldsymbol{g}}\right]_{1}=\boldsymbol{V}_{1} /\left(A_{\boldsymbol{g}}\right)_{1}=\boldsymbol{k}^{d+1-3}=$ $k^{d-2}$. Therefore, $V / A_{\boldsymbol{g}}$ is minimally generated by $d-2$ elements of degree 1 as a graded $T$-module.

The rings $A_{\boldsymbol{g}}$ and $\boldsymbol{V}$ are Cohen-Macaulay of dimension 2 ; hence, $A_{\boldsymbol{g}}$ and $\boldsymbol{V}$ are Cohen-Macaulay $T$-modules of dimension 2. It follows that each of these $T$-modules is perfect of projective dimension equal to 1 . Consider the $T$-free resolutions of these modules:

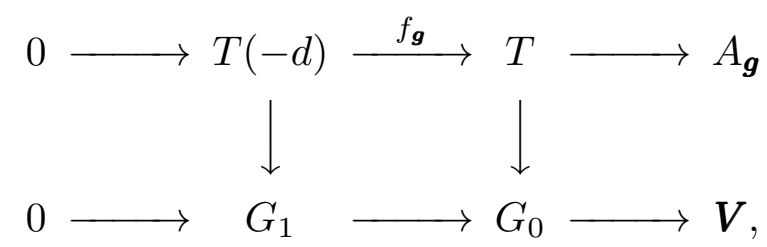

for some graded free $T$-modules $G_{0}$ and $G_{1}$. The mapping cone of (8.3) gives a $T$ free resolution of $\boldsymbol{V} / A_{\boldsymbol{g}}$ of length 2. On the other hand, grade $\operatorname{ann}_{T}\left(\boldsymbol{V} / A_{\boldsymbol{g}}\right)$ is at least two. Indeed, $f_{\boldsymbol{g}}$ is a prime element of $T$ which is in $\operatorname{ann}_{T}\left(\boldsymbol{V} / A_{\boldsymbol{g}}\right)$. Furthermore, the extension $A_{\boldsymbol{g}} \subseteq \boldsymbol{V}$ is birational and $\boldsymbol{V}$ is finitely generated as an $A_{\boldsymbol{g}}$-module; so there is a non-zero homogeneous element of $A_{\boldsymbol{g}}$ which is in $\operatorname{ann}_{T}\left(\boldsymbol{V} / A_{\boldsymbol{g}}\right)$. Lift this element back to $T$ to complete a regular sequence of length 2 on $T$ in $\operatorname{ann}_{T}\left(\boldsymbol{V} / A_{\boldsymbol{g}}\right)$. It follows that $\boldsymbol{V} / A_{\boldsymbol{g}}$ is a perfect $T$-module with $\operatorname{pd}_{T}\left(\boldsymbol{V} / A_{\boldsymbol{g}}\right)=\operatorname{grade} \operatorname{ann}_{T}\left(\boldsymbol{V} / A_{\boldsymbol{g}}\right)=2$. In particular, $V / A_{\boldsymbol{g}}$ is a Cohen-Macualay $T$-module of dimension 1 and the $T$-free resolution of $\boldsymbol{V} / A_{\boldsymbol{g}}$ has the form

$$
0 \rightarrow T(-d) \rightarrow F_{1} \rightarrow T^{d-2}(-1) \rightarrow V / A_{g}
$$

for some graded free $T$-module $F_{1}$. Dualize the above exact sequence and shift by $d$ in order to obtain the exact sequence:

$$
0 \rightarrow T^{d-2}(-d+1) \rightarrow F_{1}^{*}(-d) \rightarrow T \rightarrow T / H \rightarrow 0
$$

where $H$ is a perfect ideal of height 2. Since $\operatorname{rank} F_{1}^{*}=d-1$, we have $\mu(H)=d-1$; and hence, the initial degree of $H$ is at least $d-2$ by the Hilbert-Burch Theorem and therefore,

$$
F_{1}^{*}(-d)=T^{d-1}(-d+2) .
$$

Finally, $\boldsymbol{V} / A_{\boldsymbol{g}} \cong \omega_{T / H}$; and therefore, $\boldsymbol{V} / A_{\boldsymbol{g}}$ is a faithful $T / H$-module. Thus, $H=$ $\operatorname{ann}_{T}\left(\boldsymbol{V} / A_{\boldsymbol{g}}\right)$. But the later is $L_{\boldsymbol{g}}$ by the definition of $L_{\boldsymbol{g}}$; therefore, $H=L_{\boldsymbol{g}}$. 
Corollary 8.4. Adopt Data 8.1. The following statements hold.

(a) The ring $A_{\boldsymbol{g}} / \mathfrak{c}_{\boldsymbol{g}}$ is 1-dimensional and Cohen-Macaulay of multiplicity $\left(\begin{array}{c}d-1 \\ 2\end{array}\right)$.

(b) The ring $V / \mathfrak{c}_{\boldsymbol{g}}$ is 1-dimensional and Cohen-Macaulay of multiplicity $(d-1)(d-2)$.

Proof. Assertion (a) follows from the resolution in Lemma 8.2, using for instance [20]. We prove (b). By Lemma 8.2 we have the short exact sequence

$$
0 \rightarrow A_{\boldsymbol{g}} / \mathfrak{c}_{\boldsymbol{g}} \rightarrow V / \mathfrak{c}_{\boldsymbol{g}} \rightarrow \boldsymbol{V} / A_{\boldsymbol{g}} \cong \omega_{A_{\boldsymbol{g}} / \mathfrak{c}_{\boldsymbol{g}}} \rightarrow 0 .
$$

Since $\omega_{A_{\boldsymbol{g}} / \mathfrak{c}_{\boldsymbol{g}}}$ is a Cohen-Macaulay module of dimension one and multiplicity $\left(\begin{array}{c}d-1 \\ 2\end{array}\right)$ by part (a), it follows that $V / \mathfrak{c}_{\boldsymbol{g}}$ is a Cohen-Macaulay 1-dimensional ring of multiplicity $2\left(\begin{array}{c}d-1 \\ 2\end{array}\right)=(d-1)(d-2)$.

We define the matrix $M_{\boldsymbol{g}}^{\prime}$ which appears in the next result. Let $\varphi_{\boldsymbol{g}}$ be a homogeneous Hilbert-Burch matrix for the row vector $\left[g_{1}, g_{2}, g_{3}\right]$ of degree $\left(d_{1}, d_{2}\right)$ and let

$$
N=\left[\begin{array}{cc}
\rho^{\left(d_{2}\right)} & 0 \\
0 & \rho^{\left(d_{1}\right)}
\end{array}\right],
$$

for $\rho^{(i)}$ as given in (3.1). Each entry in the row vector $\left[T_{1}, T_{2}, T_{3}\right] \varphi_{\boldsymbol{g}} N$ is a homogeneous form of degree 1 in the $T$ 's and degree $d$ in $x, y$. Define $M_{\boldsymbol{g}}^{\prime}$ to be the matrix with homogeneous linear entries from $T$ which satisfies the equation

$$
\left[T_{1}, T_{2}, T_{3}\right] \varphi_{\boldsymbol{g}} N=\rho^{(d)} M_{\boldsymbol{g}}^{\prime}
$$

Proposition 8.6. Adopt Data 8.1. A homogeneous T-presentation of $\boldsymbol{V}_{+}$is given by

$$
T^{d+2}(-2) \stackrel{M_{\boldsymbol{g}}^{\prime}}{\longrightarrow} T^{d+1}(-1) \rightarrow V_{+} \rightarrow 0
$$

Proof. The sequence $0 \rightarrow V_{+} \rightarrow \boldsymbol{V} \rightarrow \boldsymbol{k} \rightarrow 0$ shows that $\operatorname{reg}_{T} \boldsymbol{V}_{+}=1$; so, $\boldsymbol{V}_{+}$as a $T$-module is minimally generated by the monomials $y^{d}, \ldots, x^{d}$ and the relations between those generators have degree 1 . Let

$$
\ell=\left[\begin{array}{c}
\ell_{0} \\
\vdots \\
\ell_{d}
\end{array}\right]
$$

be a matrix of linear forms from $T$, and let $\Lambda$ be a matrix of scalars with

$$
\boldsymbol{\ell}=\Lambda\left[\begin{array}{l}
T_{1} \\
T_{2} \\
T_{3}
\end{array}\right]
$$


The polynomial $T_{i}$ of $T$ acts like $g_{i}$ on $A_{\boldsymbol{g}}$; so

$$
\rho^{(d)} \boldsymbol{\ell}=0 \text { in } \boldsymbol{V}_{+} \Longleftrightarrow \rho^{(d)} \Lambda\left[\begin{array}{l}
g_{1} \\
g_{2} \\
g_{3}
\end{array}\right]=0 \text { in } B \text {. }
$$

The matrix $\varphi_{\boldsymbol{g}}$ is a Hilbert-Burch matrix for the row vector $\left[g_{1}, g_{2}, g_{3}\right]$; so $\rho^{(d)} \boldsymbol{\ell}=0$ in $V_{+}$if and only if there exist homogeneous forms $F_{1}$ and $F_{2}$ in $B$ with $\operatorname{deg} F_{1}=d_{2}$, $\operatorname{deg} F_{2}=d_{1}$, and

$$
\Lambda^{\mathrm{T}}\left(\rho^{(d)}\right)^{\mathrm{T}}=\varphi_{\boldsymbol{g}}\left[\begin{array}{l}
F_{1} \\
F_{2}
\end{array}\right]
$$

The vector $\left[\begin{array}{l}F_{1} \\ F_{2}\end{array}\right]$ is equal to $N \boldsymbol{F}$ for some columns vector of scalars $\boldsymbol{F}$. We have

$$
\begin{aligned}
\rho^{(d)} \boldsymbol{\ell}=0 \text { in } \boldsymbol{V}_{+} & \Longleftrightarrow \exists \boldsymbol{F} \text { with } \Lambda^{\mathrm{T}}\left(\rho^{(d)}\right)^{\mathrm{T}}=\varphi_{\boldsymbol{g}} N \boldsymbol{F} \\
& \Longleftrightarrow \exists \boldsymbol{F} \text { with }\left[T_{1}, T_{2}, T_{3}\right] \Lambda^{\mathrm{T}}\left(\rho^{(d)}\right)^{\mathrm{T}}=\left[T_{1}, T_{2}, T_{3}\right] \varphi_{\boldsymbol{g}} N \boldsymbol{F} \\
& \Longleftrightarrow \exists \boldsymbol{F} \text { with } \rho^{(d)} \Lambda\left[\begin{array}{c}
T_{1} \\
T_{2} \\
T_{3}
\end{array}\right]=\rho^{(d)} M_{\boldsymbol{g}}^{\prime} \boldsymbol{F} \\
& \Longleftrightarrow \exists \boldsymbol{F} \text { with } \boldsymbol{\ell}=M_{\boldsymbol{g}}^{\prime} \boldsymbol{F} .
\end{aligned}
$$

Notation set up. Complete $g_{1}, g_{2}, g_{3}$ to a $\boldsymbol{k}$-basis $g_{1}, g_{2}, g_{3}, \ldots, g_{d+1}$ of $B_{d}=\boldsymbol{V}_{1}$ and let $E_{\boldsymbol{g}}^{\prime}$ be an element of $\mathrm{GL}_{d+1}(\boldsymbol{k})$ with

$$
\left[g_{1}, g_{2}, g_{3}, \ldots, g_{d+1}\right]=\rho^{(d)} E_{\boldsymbol{g}}^{\prime}
$$

Let $E_{\boldsymbol{g}}=\operatorname{Adj}\left(E_{\boldsymbol{g}}^{\prime}\right)$ and $M_{\boldsymbol{g}}$ be the matrix obtained from $E_{\boldsymbol{g}} M_{\boldsymbol{g}}^{\prime}$ by deleting the first three rows.

Corollary 8.8. Adopt Data 8.1. The following statements hold.

(a) The matrix $M_{\boldsymbol{g}}$ is a presentation matrix for the $T$-module $\boldsymbol{V} / A_{\boldsymbol{g}}$.

(b) The ideals $L_{\boldsymbol{g}}$ and $\mathfrak{c}_{\boldsymbol{g}}$ of $T$ and $A_{\boldsymbol{g}}$ are equal to $I_{d-2}\left(M_{\boldsymbol{g}}\right)$ and $I_{d-2}\left(M_{\boldsymbol{g}}\right) A_{\boldsymbol{g}}$, respectively.

Remark. To obtain $\mathfrak{c}_{\boldsymbol{g}}$ from $L_{\boldsymbol{g}}$, we substitute $g_{i}$ in place of $T_{i}$.

Proof. (a) Notice that $E_{\boldsymbol{g}} M_{\boldsymbol{g}}^{\prime}$ is a matrix of relations on the row vector $\left[g_{1}, \ldots, g_{d+1}\right]$; hence, $M_{\boldsymbol{g}}$ is a presentation matrix for

$$
\frac{T g_{1}+\cdots+T g_{d+1}}{T g_{1}+T g_{2}+T g_{3}}=V / A_{g}
$$

(b) The proof of Lemma 8.2 shows that $L_{\boldsymbol{g}}=I_{d-2}\left(M_{\boldsymbol{g}}\right)$ since $M_{\boldsymbol{g}}$ is a presentation matrix for $\boldsymbol{V} / A_{\boldsymbol{g}}$. 
Theorem 8.9. Adopt Data 8.1. The ideal $\mathfrak{c}_{\boldsymbol{g}} B$ is equal to $c_{\boldsymbol{g}}(x, y)^{d-2}$, for some form $c_{\boldsymbol{g}}$ in $B$ of degree $(d-1)(d-2)$.

Proof. Since the $\boldsymbol{V}$-module $B$ contains $\boldsymbol{V}$ as a direct summand, we have $\mathfrak{c}_{\boldsymbol{g}} B \cap \boldsymbol{V}=$ $\mathfrak{c}_{\boldsymbol{g}}$; and hence, $\boldsymbol{V} / \mathfrak{c}_{\boldsymbol{g}} \hookrightarrow B / \mathfrak{c}_{\boldsymbol{g}} B$. Therefore,

$$
\boldsymbol{V} / \mathfrak{c}_{\boldsymbol{g}}=\left(B / \mathfrak{c}_{\boldsymbol{g}} B\right)^{(d)}
$$

that is, $\boldsymbol{V} / \mathfrak{c}_{\boldsymbol{g}}$ is the $d^{\text {th }}$ Veronese subring of $B / \mathfrak{c}_{\boldsymbol{g}} B$. It follows that $B / \mathfrak{c}_{\boldsymbol{g}} B$ is also a one-dimensional ring; furthermore, the Hilbert functions of $B / \mathfrak{c}_{\boldsymbol{g}} B$ and $V / \mathfrak{c}_{\boldsymbol{g}}$ eventually take the same value. It follows that $B / \mathfrak{c}_{\boldsymbol{g}} B$ and $\boldsymbol{V} / \mathfrak{c}_{\boldsymbol{g}}$ have the same multiplicity and, according to Corollary $8.4(\mathrm{~b})$, this multiplicity is $(d-1)(d-2)$. The ideal $\mathfrak{c}_{\boldsymbol{g}} B$ has height one; so we can write $\mathfrak{c}_{\boldsymbol{g}} B=c_{\boldsymbol{g}} J$ for some form $c_{\boldsymbol{g}}$ of $B$ and some ideal $J$ of $B$ of height at least 2 . We have

$$
(d-1)(d-2)=e\left(B / \mathfrak{c}_{\boldsymbol{g}} B\right)=e\left(B /\left(c_{\boldsymbol{g}}\right)\right)=\operatorname{deg} c_{\boldsymbol{g}}
$$

The ideal $\mathfrak{c}_{\boldsymbol{g}} B$ is generated by $I_{d-2}\left(M_{\boldsymbol{g}}\right) B$, see Corollary 8.8; and therefore, the initial degree of $\mathfrak{c}_{\boldsymbol{g}} B$ is $d(d-2)$. Compare initial degrees to see that $J$ is generated by forms of degree $(d-2)$.

We have $\mathfrak{c}_{\boldsymbol{g}}=L_{\boldsymbol{g}} /\left(f_{\boldsymbol{g}}\right)$. Recall that $L_{\boldsymbol{g}}$ is minimally generated as a $T$-module by $d-1$ forms of degree $d-2$ (see Lemma 8.2) and $f_{\boldsymbol{g}}$ has degree $d$. Hence, as an $A_{\boldsymbol{g}}$-module, $\mathfrak{c}_{\boldsymbol{g}}$ is minimally generated by $d-1$ forms of the same degree; and therefore, as a $B$-module $\mathfrak{c}_{\boldsymbol{g}}$ is minimally generated by $d-1$ forms because $B_{0}=\left(A_{\boldsymbol{g}}\right)_{0}$. Thus, $J$ is minimally generated by $d-1$ forms of degree $d-2$; and therefore, $J=(x, y)^{d-2}$.

Theorem 8.11. Adopt Data 8.1 with $\boldsymbol{k}$ an algebraically closed field. Factor the polynomial $c_{\boldsymbol{g}}$ of Theorem 8.9 as $\prod_{j=1}^{n} \ell_{j}^{t_{j}}$, where the $\ell_{j}$ are pairwise linearly independent linear forms in $B$ and the $t_{j}$ are positive integers.

(a) The sets $V\left(\ell_{1}\right) \cup \cdots \cup V\left(\ell_{n}\right)$ and $\Psi_{\boldsymbol{g}}^{-1}\left(\operatorname{Sing}\left(\mathcal{C}_{\boldsymbol{g}}\right)\right)$ are equal, where the curve $\mathcal{C}_{\boldsymbol{g}}$ and the parameterization $\Psi_{\boldsymbol{g}}: \mathbb{P}^{1} \rightarrow \mathbb{P}^{2}$ are described in Definition 5.3 , and $\operatorname{Sing}\left(\mathcal{C}_{\boldsymbol{g}}\right)$ is the singular locus of $\mathcal{C}_{\boldsymbol{g}}$.

(b) The sets $V\left(\ell_{1} B \cap A_{\boldsymbol{g}}\right) \cup \cdots \cup V\left(\ell_{n} B \cap A_{\boldsymbol{g}}\right)$ and $\operatorname{Sing}\left(\mathcal{C}_{\boldsymbol{g}}\right)$ are equal.

(c) If $P \in \operatorname{Sing}\left(\mathcal{C}_{\boldsymbol{g}}\right)$, then

$$
\delta_{P}=\frac{1}{2} \sum_{\left\{j \mid P=V\left(\ell_{j} B \cap A_{\boldsymbol{g}}\right)\right\}} t_{j} .
$$

(d) The equation $t_{j}=2 \delta_{\ell_{j}}+i_{\ell_{j}}$ holds, where $\delta_{\ell_{j}}$ is the $\delta$-invariant of the branch $\ell_{j}$ and $i_{\ell_{j}}$ is the intersection multiplicity of $\ell_{j}$ with the other branches. (In the language of Lemma 1.7, $\delta_{\ell_{j}}=\delta\left(\hat{R} / J_{j}\right)=\lambda\left(\overline{\left(\hat{R} / J_{j}\right)} /\left(\hat{R} / J_{j}\right)\right)$ and $i_{\ell_{j}}=\sum_{i \neq j} \lambda\left(\hat{R} /\left(J_{i}, J_{j}\right)\right)$, where $\lambda=\lambda_{\hat{R}}$ is length.) 
Proof. For (b), notice that $\operatorname{Sing}\left(\mathcal{C}_{\boldsymbol{g}}\right)=V\left(\mathfrak{c}_{\boldsymbol{g}}\right) \subseteq \operatorname{Proj}\left(A_{\boldsymbol{g}}\right)=\mathcal{C}_{\boldsymbol{g}}$; otherwise, (a) and (b) are clear. We prove (c). Let $p$ be the prime ideal of $A_{\boldsymbol{g}}$ which corresponds to the point $P$ on $\mathcal{C}_{\boldsymbol{g}}$. We have have

$$
\delta_{P}=\lambda\left(\overline{\mathcal{O}_{\mathcal{C}_{\boldsymbol{g}}, p}} / \mathcal{O}_{\mathcal{C}_{\boldsymbol{g}}, p}\right)=\lambda\left(\overline{\left(A_{\boldsymbol{g}}\right)_{p}} /\left(A_{\boldsymbol{g}}\right)_{p}\right)=\frac{1}{2} \lambda\left(\boldsymbol{V}_{p} /\left(\mathfrak{c}_{\boldsymbol{g}}\right)_{p}\right) .
$$

The right most equality holds because the ring $\left(A_{\boldsymbol{g}}\right)_{p}$ is Gorenstein and $V_{p}$ is the integral closure of $\left(A_{\boldsymbol{g}}\right)_{p}$. Observe that $\lambda\left(\boldsymbol{V}_{p} /\left(\mathfrak{c}_{\boldsymbol{g}}\right)_{p}\right)=\lambda\left(B_{p} / \mathfrak{c}_{\boldsymbol{g}} B_{p}\right)$ since, according to (8.10), the rings $\boldsymbol{V} / \mathfrak{c}_{\boldsymbol{g}} \subseteq B / \mathfrak{c}_{\boldsymbol{g}} B$ are equal on the punctured spectrum. We have

$$
\begin{aligned}
\delta\left(\mathcal{O}_{\mathcal{C}_{\boldsymbol{g}}, p}\right) & =\frac{1}{2} \lambda\left(B_{p} / \mathfrak{c}_{\boldsymbol{g}} B_{p}\right)=\frac{1}{2} \lambda\left(B_{p} / c_{\boldsymbol{g}} B_{p}\right) \\
& =\frac{1}{2} \sum_{\left\{j \mid p=\ell_{j} B \cap A_{\boldsymbol{g}}\right\}} t_{j}=\frac{1}{2} \sum_{\left\{j \mid P=V\left(\ell_{j} B \cap A_{\boldsymbol{g}}\right)\right\}} t_{j} .
\end{aligned}
$$

The proof of (d) may be found in [23, Thm. 17.12].

Theorem 8.12. Adopt Data 8.1 with $d=4$ and $\boldsymbol{k}$ an algebraically closed field. Let $\%$ be one of the 13 possible configurations of singularities on a rational plane quartic as listed in (0.3). If $\boldsymbol{g} \in \mathbb{T}_{d}$ and the singularities on $\mathcal{C}_{\boldsymbol{g}}$ are described by $\%$, then $c_{\boldsymbol{g}}$ has the form described in the following chart. In each case, the linear forms $\left\{\ell_{j}\right\}$ in $\boldsymbol{k}[x, y]$ are pairwise linearly independent.

$$
\begin{array}{rr}
\multicolumn{1}{c}{\%} & \multicolumn{1}{c}{c_{\boldsymbol{g}}} \\
(2: 1,1)^{3} & \ell_{1} \ell_{2} \ell_{3} \ell_{4} \ell_{5} \ell_{6} \\
(2: 2: 1,1),(2: 1,1) & \ell_{1}^{2} \ell_{2}^{2} \ell_{3} \ell_{4} \\
(2: 1,1)^{2},(2: 1) & \ell_{1}^{2} \ell_{2} \ell_{3} \ell_{4} \ell_{5} \\
(2: 2: 2: 1,1) & \ell_{1}^{3} \ell_{2}^{3} \\
(2: 2: 1),(2: 1,1) & \ell_{1}^{4} \ell_{2} \ell_{3} \\
(2: 2: 1,1),(2: 1) & \ell_{1}^{2} \ell_{2}^{2} \ell_{3}^{2} \\
(2: 1,1),(2: 1)^{2} & \ell_{1}^{2} \ell_{2}^{2} \ell_{3} \ell_{4} \\
(2: 2: 2: 1) & \ell_{1}^{6} \\
(2: 2: 1),(2: 1) & \ell_{1}^{4} \ell_{2}^{2} \\
(2: 1)^{3} & \ell_{1}^{2} \ell_{2}^{2} \ell_{3}^{2} \\
(3: 1,1,1) & \ell_{1}^{2} \ell_{2}^{2} \ell_{3}^{2} \\
(3: 1,1) & \ell_{1}^{4} \ell_{2}^{2} \\
(3: 1) & \ell_{1}^{6}
\end{array}
$$


Proof. Throughout this discussion, $\mathcal{C}=\mathcal{C}_{\boldsymbol{g}}, c=c_{\boldsymbol{g}}$, and the singularities on $\mathcal{C}_{\boldsymbol{g}}$ are described by $\%$. The polynomial $c$ is a homogeneous form of degree $(d-2)(d-1)=6$.

If $\%=(2: 2: 2: 1)$, then there is only one singular point on $\mathcal{C}$ and there is only one branch at this singular point; thus, $c=\ell_{1}^{6}$.

If $\%=(2: 2: 2: 1,1)$, then there only one singular point $P$ on $\mathcal{C}$. This singularity has two branches. Each branch is smooth (i.e., $\delta_{\ell_{j}}=0$ ) because the multiplicity of the singularity is 2 . Thus, $t_{j}=i_{\ell_{j}}$ and $i_{\ell_{1}}=i_{\ell_{2}}$. Notice that

$$
i_{\ell_{1}}=\lambda\left(\widehat{\mathcal{O}}_{\mathcal{C}, P} /\left(q_{1}+q_{2}\right)\right)=i_{\ell_{2}}
$$

where $q_{j}$ is the minimal prime ideal of $\widehat{\mathcal{O}}_{\mathcal{C}, P}$ which corresponds to $\ell_{j}$. Thus, $c=\ell_{1}^{3} \ell_{2}^{3}$.

If $\%=(2: 2: 1),(2: 1)$, then there are two singular points, $P_{1}, P_{2}$, on $\mathcal{C}$, each with one branch: $q_{1} \leftrightarrow \ell_{1}$ and $q_{2} \leftrightarrow \ell_{2}$. Theorem 8.11 (c) shows that $t_{1}=4$ and $t_{2}=2$; and therefore, $c=\ell_{1}^{4} \ell_{2}^{2}$.

If $\%=(2: 2: 1),(2: 1,1)$, then there are two singular points, $P_{1}, P_{2}$, on $\mathcal{C}$. The singular point $P_{1}$ has one branch $q_{1} \leftrightarrow \ell_{1}$ and $\delta_{P_{1}}=2$. The corresponding factor of $c$ is $\ell_{1}^{4}$ by part (c) of Theorem 8.11. The singular point $P_{2}$ has two branches: $q_{2} \leftrightarrow \ell_{2}$ and $q_{3} \leftrightarrow \ell_{3}$. The factors $\ell_{2}$ and $\ell_{3}$ of $c$ appear with exponent 1 because $c$ is a form of degree 6 and there is no room for any higher exponent. Thus, $c=\ell_{1}^{4} \ell_{2} \ell_{3}$.

If $\%=(2: 2: 1,1),(2: 1)$, then there are two singular points, $P_{1}, P_{2}$, on $\mathcal{C}$. The singular point $P_{1}$ has two branches: $q_{1} \leftrightarrow \ell_{1}$ and $q_{2} \leftrightarrow \ell_{2}$, with $\delta_{P_{1}}=2$. The exponents $t_{1}$ and $t_{2}$ of $\ell_{1}$ and $\ell_{2}$ in $c$ satisfy $t_{1}+t_{2}=4$ by part (c) of Theorem 8.11. The two branches are smooth; so $t_{1}=i_{1}=i_{2}=t_{2}$; hence, $t_{1}=t_{2}=2$. The singular point $P_{2}$ has one branch: $q_{3} \leftrightarrow \ell_{3}$. The fact that $\operatorname{deg} c=6$ forces $t_{3}$ to be 2. In this case, we have $c=\ell_{1}^{2} \ell_{2}^{2} \ell_{3}^{2}$.

One may proceed through the other eight cases using the same techniques.

Definition 8.13. Let $d$ be an even integer. Recall the open subset $\mathbb{B}_{d}$ of $\mathbb{A}_{d}$ from Definition 5.4. For each pair of indices $\boldsymbol{r}=\left(r_{1}, r_{2}\right)$ with $1 \leq r_{1} \leq r_{2} \leq d$, define $\mathbb{B}_{d}^{(j, \boldsymbol{r})}$ to be the set of all $\boldsymbol{g} \in \mathbb{B}_{d}$ with

$$
\lambda_{0, j} \operatorname{det}\left[\begin{array}{ll}
\lambda_{r_{1}, c_{1}} & \lambda_{r_{1}, c_{2}} \\
\lambda_{r_{2}, c_{1}} & \lambda_{r_{2}, c_{2}}
\end{array}\right] \neq 0
$$

where $\left\{j, c_{1}, c_{2}\right\}=\{1,2,3\}$ and $\lambda_{\boldsymbol{g}}$ is given in Remark 5.2.

Remark 8.14. The sets $\cup_{j, \boldsymbol{r}} \mathbb{B}_{d}^{(j, \boldsymbol{r})}$ form an open cover of $\mathbb{B}_{d}$.

Notation 8.15. Recall $\boldsymbol{z}, \boldsymbol{R}$, and $\boldsymbol{S}$ as given in Convention (1) of 5.15 and view $\boldsymbol{V}[\boldsymbol{z}]$ as a subring of $\boldsymbol{S}$. Recall also Convention (2) of 5.15. Let $\lambda$ be in $\mathbb{A}^{3 d+3}$. The ring $\boldsymbol{R}_{\lambda}$ is defined to be $\boldsymbol{R} /\left(\left\{z_{i, j}-\lambda_{i, j}\right\}\right)$. If $S$ is any $\boldsymbol{R}$-algebra, then $S_{\lambda}$ is defined 
to be $S \otimes_{\boldsymbol{R}} \boldsymbol{R}_{\lambda}$. Furthermore, if $G$ is an element of $S$, then $\left.G\right|_{\lambda}$ is the image of $G$ in $S_{\lambda}$. We use the same "evaluation" notation for ideals of $S$ or matrices with entries in $S$. In other words, if $I$ is an ideal of $S$, then $\left.I\right|_{\lambda}$ is the image of $I$ in $S_{\lambda}$ and if $E$ is a matrix with entries in $S$, then $\left.E\right|_{\lambda}$ is the image of $E$ with entries in $S_{\lambda}$.

Lemma 8.16. Adopt Data 8.1 with d even. Fix one of the pairs $(j, \boldsymbol{r})$ as described in Remark 8.14. Then there exists an ideal $C \subseteq \boldsymbol{V}[\boldsymbol{z}]$ so that if $\boldsymbol{g}$ is in $\mathbb{B}_{d}^{(j, \boldsymbol{r})}$, then $\left.C\right|_{\lambda_{\boldsymbol{g}}}$ is equal to the conductor $\mathfrak{c}_{\boldsymbol{g}}$.

Remark. We recall that $\left.C\right|_{\lambda_{\boldsymbol{g}}}$ is the image of $C$ in $\boldsymbol{V}[\boldsymbol{z}]_{\lambda_{\boldsymbol{g}}}=\boldsymbol{V}$.

Proof. Let $G_{k}$, for $1 \leq k \leq 3$, be the polynomials in $\boldsymbol{V}[\boldsymbol{z}]$ which are defined in (5.16), and let $G_{4}, \ldots, G_{d+1}$ be the polynomials

$$
\left\{y^{d}, x y^{d-1}, \ldots, x^{d}\right\} \backslash\left\{y^{d}, x^{r_{1}} y^{d-r_{1}}, x^{r_{2}} y^{d-r_{2}}\right\}
$$

in $\boldsymbol{V}$. If $\boldsymbol{g} \in \mathbb{B}_{d}^{(j, \boldsymbol{r})}$, then the definition of $\mathbb{B}_{d}^{(j, \boldsymbol{r})}$ shows that

$$
\left.G_{1}\right|_{\lambda_{g}},\left.G_{2}\right|_{\lambda_{g}},\left.G_{3}\right|_{\lambda_{g}}, G_{4}, \ldots, G_{d+1}
$$

is a basis for $B_{d}=\left[(\boldsymbol{V}[\boldsymbol{z}])_{\lambda_{\boldsymbol{g}}}\right]_{d}$. Let $E^{\prime}$ be the following $(d+1) \times(d+1)$ matrix over $V[\boldsymbol{z}]$

$$
E^{\prime}=\left[\begin{array}{ccc|c}
z_{1,1} & z_{1,2} & z_{1,3} & \\
z_{2,1} & z_{2,2} & z_{3,3} & \\
\vdots & \vdots & \vdots & * \\
\vdots & \vdots & \vdots & \\
z_{d, 1} & z_{d, 2} & z_{d, 3} &
\end{array}\right]
$$

with

$$
\left[G_{1}, \ldots, G_{d+1}\right]=\rho^{(d)} E^{\prime}
$$

and each entry of $*$ is either 0 or 1 . (The matrix $\rho^{(d)}$ is defined in (3.1).) If $\boldsymbol{g} \in \mathbb{B}_{d}^{(j, \boldsymbol{r})}$, then the image, $\left.E^{\prime}\right|_{\lambda_{\boldsymbol{g}}}$, of $E^{\prime}$ in $\boldsymbol{V}[\boldsymbol{z}]_{\lambda_{\boldsymbol{g}}}$ is in $\mathrm{GL}_{d+1}(\boldsymbol{k})$ and the map $\boldsymbol{V}[\boldsymbol{z}] \rightarrow \boldsymbol{V}[\boldsymbol{z}]_{\lambda_{\boldsymbol{g}}}=\boldsymbol{V}$ sends (8.17) to (8.7).

Recall the matrix $\boldsymbol{d}_{2}^{(j)}$ of Theorem 5.30. If $\boldsymbol{g} \in \mathbb{B}_{d}^{(j, \boldsymbol{r})}$, then $\left.\boldsymbol{d}_{2}^{(j)}\right|_{\lambda_{\boldsymbol{g}}}$ is a HilbertBurch matrix for the row vector $\left[g_{1}, g_{2}, g_{3}\right]$. Form the matrix $M^{\prime}$ which satisfies

$$
\left[\begin{array}{lll}
T_{1} & T_{2} & T_{3}
\end{array}\right] \boldsymbol{d}_{2}^{(j)}\left[\begin{array}{cc}
\rho^{(d / 2)} & 0 \\
0 & \rho^{(d / 2)}
\end{array}\right]=\rho^{(d)} M^{\prime}
$$


exactly as was done in (8.5). Each entry of $M^{\prime}$ is a homogeneous form in $\boldsymbol{k}\left[\left\{T_{k}\right\}, z\right]$ of degree 1 in the $T$ 's and degree $3 c+1$ in the $z$ 's. The matrix $M$ is obtained from $\operatorname{Adj}\left(E^{\prime}\right) M^{\prime}$ by deleting the first three rows and substituting $G_{k}$ for $T_{k}$, for $1 \leq k \leq 3$. Let $C$ be the ideal $I_{d-2}(M)$ in $\boldsymbol{V}[\boldsymbol{z}]$. If $\boldsymbol{g} \in \mathbb{B}_{d}^{(j, \boldsymbol{r})}$, then according to Corollary 8.8, we have

$$
\left.C\right|_{\lambda_{\boldsymbol{g}}}=I_{d-2}\left(M_{\boldsymbol{g}}\right) \cdot A_{\boldsymbol{g}}=\mathfrak{c}_{\boldsymbol{g}} A_{\boldsymbol{g}}=\mathfrak{c}_{\boldsymbol{g}} V
$$

Theorem 8.18. Adopt Data 8.1 with $\boldsymbol{k}$ an algebraically closed field of characteristic zero and d even. Define

$$
X=\left\{\begin{array}{l|l}
\boldsymbol{g} \in \mathbb{B}_{d} & \begin{array}{l}
c_{\boldsymbol{g}}=\prod \ell_{j}^{t_{j}} \text { with the } \ell_{j} \text { pairwise linearly independent } \\
\text { and } t_{j} \geq 2 \text { for all } j
\end{array}
\end{array}\right\} .
$$

Then the subset $X$ of $\mathbb{B}_{d}$ is closed.

Proof. According to Remark 8.14, the sets $\cup_{j, \boldsymbol{r}} \mathbb{B}_{d}^{(j, \boldsymbol{r})}$ form an open cover of $\mathbb{B}_{d}$. Therefore, it suffices to prove that $X \cap \mathbb{B}_{d}^{(j, \boldsymbol{r})}$ is a closed subset of $\mathbb{B}_{d}^{(j, \boldsymbol{r})}$ for each pair $(j, \boldsymbol{r})$ as described in Definition 8.13. Fix such a pair $(j, \boldsymbol{r})$. By Lemma 8.16 there exists an ideal $C \subseteq \boldsymbol{V}[\boldsymbol{z}]$ so that $\left.C\right|_{\lambda_{\boldsymbol{g}}}$ is equal to $\mathfrak{c}_{\boldsymbol{g}}$ for each $\boldsymbol{g} \in \mathbb{B}_{d}^{(j, \boldsymbol{r})}$. Let $D=\boldsymbol{V}[\boldsymbol{z}] / C$. Recall from Notation 8.15 that $D_{\lambda_{\boldsymbol{g}}}$ is defined to be $D \otimes_{\boldsymbol{R}} \boldsymbol{R}_{\lambda_{g}}$. It follows that $D_{\lambda_{\boldsymbol{g}}}=V /\left(\left.C\right|_{\lambda_{\boldsymbol{g}}}\right)=V / \mathfrak{c}_{\boldsymbol{g}}$.

Theorem 8.9 ensures that $\mathfrak{c}_{\boldsymbol{g}} B=\ell_{1}^{t_{1}} \cdots \ell_{n}^{t_{n}}(x, y)^{d-2}$ for some $n$ and some pairwise linearly independent forms $\left\{\ell_{j}\right\}$. We see that

$$
t_{j} \geq 2 \text { for all } j \Longleftrightarrow \operatorname{Reg}\left(B / \mathfrak{c}_{\boldsymbol{g}} B\right)=\emptyset
$$

where $\operatorname{Reg}(R)$ is the regular locus of the ring $R$. Recall from (8.10) that $V / \mathfrak{c}_{\boldsymbol{g}}$ is equal to $\left(B / \mathfrak{c}_{\boldsymbol{g}} B\right)^{(d)}$; hence, $\operatorname{Proj}\left(\boldsymbol{V} / \mathfrak{c}_{\boldsymbol{g}}\right) \cong \operatorname{Proj}\left(B / \mathfrak{c}_{\boldsymbol{g}} B\right)$, and

$$
\operatorname{Reg}\left(B / \mathfrak{c}_{\boldsymbol{g}} B\right)=\emptyset \Longleftrightarrow \operatorname{Reg}\left(\boldsymbol{V} / \mathfrak{c}_{\boldsymbol{g}}\right)=\emptyset
$$

Apply the Jacobian criterion to see that $\operatorname{Reg}\left(\boldsymbol{V} / \mathfrak{c}_{\boldsymbol{g}}\right)=\emptyset$ if and only if Jacobian ideal $\operatorname{Jac}\left(\boldsymbol{V} / \mathfrak{c}_{\boldsymbol{g}}\right)$ is nilpotent.

Let $J=\operatorname{Fitt}_{1}\left(\Omega_{D / \boldsymbol{k}[z]}\right) \subseteq D$. Notice that $\operatorname{Jac}\left(\boldsymbol{V} / \mathfrak{c}_{\boldsymbol{g}}\right)$ is the image $\left.J\right|_{\lambda_{\boldsymbol{g}}}$ of $J$ in $D_{\lambda_{\boldsymbol{g}}}=\boldsymbol{V} / \mathfrak{c}_{\boldsymbol{g}}$. Recall from Corollary 8.4 (b) that $D_{\lambda_{\boldsymbol{g}}}$ is Cohen-Macaulay and has multiplicity $(d-1)(d-2)$ for all $\boldsymbol{g} \in \mathbb{B}_{d}^{(j, \boldsymbol{r})}$.

We claim that $\left.J\right|_{\lambda_{\boldsymbol{g}}}$ is nilpotent if and only if $\left.J\right|_{\lambda_{\boldsymbol{g}}} ^{(d-1)(d-2)}=0$. Indeed, if $\left.J\right|_{\lambda_{\boldsymbol{g}}}$ is nilpotent, then $\left.J\right|_{\lambda_{\boldsymbol{g}}} \subseteq q$ for all $q \in \min \left(D_{\lambda_{\boldsymbol{g}}}\right)$. However, the length of $\left(D_{\lambda_{\boldsymbol{g}}}\right)_{q}$ is at 
most $e\left(D_{\lambda_{\boldsymbol{g}}}\right)=(d-1)(d-2)$; hence, $q_{q}^{(d-1)(d-2)}=0$ which forces $\left(\left.J\right|_{\lambda_{\boldsymbol{g}}}\right)_{q}^{(d-1)(d-2)}$ to be zero. Since this holds for every minimal prime and $D_{\lambda_{\boldsymbol{g}}}$ is Cohen-Macaulay, we have $\left.J\right|_{\lambda_{\boldsymbol{g}}} ^{(d-1)(d-2)}=0$. Write $\mathfrak{a}=J^{(d-1)(d-2)}$. It remains to show that the set

$$
\left\{\boldsymbol{g} \in \mathbb{B}_{d}^{(j, \boldsymbol{r})}|\mathfrak{a}|_{\lambda_{\boldsymbol{g}}}=0\right\}
$$

is closed in $\mathbb{B}_{d}^{(j, \boldsymbol{r})}$.

Since the $D_{\lambda_{\boldsymbol{g}}}$ are Cohen-Macaulay rings, we have that $\left.\mathfrak{a}\right|_{\lambda_{\boldsymbol{g}}}=0$ if and only if $\operatorname{dim}\left((D / \mathfrak{a})_{\lambda_{\boldsymbol{g}}}\right)=\operatorname{dim} D_{\lambda_{\boldsymbol{g}}}$ and $e\left((D / \mathfrak{a})_{\lambda_{\boldsymbol{g}}}\right)=e\left(D_{\lambda_{\boldsymbol{g}}}\right)$. Since $\operatorname{dim} D_{\lambda_{\boldsymbol{g}}}=1$ and $e\left(D_{\lambda_{\boldsymbol{g}}}\right)=(d-1)(d-2)$ for all $\boldsymbol{g} \in \mathbb{B}_{d}^{(j, \boldsymbol{r})}$, we conclude that set described in (8.19) is equal to

$$
\left\{\boldsymbol{g} \in \mathbb{B}_{d}^{(j, \boldsymbol{r})} \mid \operatorname{dim}\left((D / \mathfrak{a})_{\lambda_{\boldsymbol{g}}}\right) \geq 1\right\} \cap\left\{\boldsymbol{g} \in \mathbb{B}_{d}^{(j, \boldsymbol{r})} \mid e\left((D / \mathfrak{a})_{\lambda_{\boldsymbol{g}}}\right) \geq(d-1)(d-2)\right\} .
$$

But the latter set is closed in $\mathbb{B}_{d}^{(j, \boldsymbol{r})}$ by the upper semi-continuity of dimension and multiplicity; in other words, we apply Theorem 5.13 to $\boldsymbol{R} \rightarrow D / \mathfrak{a}$.

Proposition 8.20. Adopt Data 8.1 with $\boldsymbol{k}$ an algebraically closed field of characteristic zero and d even. For each positive integer $i$, define

$$
X_{i}=\left\{\begin{array}{l|l}
\boldsymbol{g} \in \mathbb{B}_{d} & \begin{array}{l}
c_{\boldsymbol{g}}=\prod \ell_{h}^{t_{h}} \text { with the } \ell_{h} \text { pairwise linearly independent } \\
\text { and } t_{k} \geq i \text { for some } k
\end{array}
\end{array}\right\} .
$$

Then each subset $X_{i}$ of $\mathbb{B}_{d}$ is closed.

Proof. Fix $(j, \boldsymbol{r})$ as in the proof of Theorem 8.18. We prove that $X_{i} \cap \mathbb{B}_{d}^{(j, \boldsymbol{r})}$ is closed in $\mathbb{B}_{d}^{(j, \boldsymbol{r})}$. Keep the ideal $C$ of $\boldsymbol{V}[\boldsymbol{z}]$ from the proof of Theorem 8.18. We continue to have $\left.C\right|_{\lambda_{\boldsymbol{g}}}$ equal to the ideal $\mathfrak{c}_{g}$ of $\boldsymbol{V}[\boldsymbol{z}]$ for all $\boldsymbol{g}$ in $\mathbb{B}_{d}^{(j, \boldsymbol{r})}$. Let $C_{1}, \ldots, C_{s}$, for some $s$, be the generators for $C$ in $\boldsymbol{V}[\boldsymbol{z}]$ as described in Lemma 8.16. Recall that $V \subseteq B$. Let $\mathfrak{b}_{i}$ be the ideal of $B[z]$ which is generated by $C_{1}, \ldots, C_{s}$ together with all partial derivatives $\frac{\partial^{k} C_{w}}{\partial^{k} x \partial^{k} y}$ with $1 \leq w \leq s, 1 \leq k \leq i-1$, and $k_{1}+k_{2}=k$. Define the ring $\mathfrak{B}_{i}=B[\boldsymbol{z}] / \mathfrak{b}_{i}$.

Let $\boldsymbol{g} \in \mathbb{B}_{d}^{(j, \boldsymbol{r})}$. We have $\left(\mathfrak{B}_{1}\right)_{\lambda_{\boldsymbol{g}}}=B / \mathfrak{c}_{\boldsymbol{g}} B$. We saw in Theorem 8.9 that $\mathfrak{c}_{\boldsymbol{g}} B=$ $\prod \ell_{h}^{t_{h}}(x, y)^{d-2} B$ where $\sum t_{h}=(d-1)(d-2)$ and the $\ell_{h}$ are pairwise linearly independent linear forms in $B$. It is clear that $\operatorname{dim}\left(\mathfrak{B}_{1}\right)_{\lambda_{\boldsymbol{g}}}=1$. The definition of the ring $\mathfrak{B}_{i}$ shows that $\left(\mathfrak{B}_{i}\right)_{\lambda_{\boldsymbol{g}}}=B /\left(\mathfrak{c}_{\boldsymbol{g}} B+\mathfrak{d} B\right)$, where $\mathfrak{d}$ is the ideal of $B$ generated by all partial derivatives $\frac{\partial^{k} \Delta}{\partial^{k} x \partial^{k_{2} y}}$ as $\Delta$ varies over all listed generators of $\mathfrak{c}_{\boldsymbol{g}} B$ and the parameters satisfy $1 \leq k_{1}+k_{2}=k \leq i-1$. We see that if there exists $h$ with $t_{h} \geq i-1$, then $0 \leq \operatorname{dim}\left(\mathfrak{B}_{i}\right)_{\lambda_{\boldsymbol{g}}} \leq 1$ and

$$
\text { there exists } k \text { with } t_{k} \geq i \Longleftrightarrow \operatorname{dim}\left(\mathfrak{B}_{i}\right)_{\lambda_{\boldsymbol{g}}}=1 \text {. }
$$


Theorem 5.13 shows that for each index $i,\left\{\lambda \in \mathbb{A}^{3 d+3} \mid \operatorname{dim}\left(\mathfrak{B}_{i}\right)_{\lambda} \geq 1\right\}$ is a closed subset of $\mathbb{A}^{3 d+3}$. It follows that $X_{i} \cap \mathbb{B}_{d}^{(j, \boldsymbol{r})}$, which is equal to

$$
\left\{\boldsymbol{g} \in \mathbb{B}_{d}^{(j, \boldsymbol{r})} \mid \operatorname{dim}\left(\mathfrak{B}_{i}\right)_{\lambda_{\boldsymbol{g}}} \geq 1\right\}
$$

is a closed subset of $\mathbb{B}_{d}^{(j, \boldsymbol{r})}$.

\section{SECTION 9. RATIONAL Plane QUARTICS: A STRATIFICATION AND THE CORRESPONDENCE BETWEEN THE HILBERT-BURCH MATRICES AND THE CONFIGURATION OF SINGULARITIES.}

The configuration of all singularities that can appear on, or infinitely near, a rational plane quartic are completely determined by two classical formulas:

$$
g=\left(\begin{array}{c}
d-1 \\
2
\end{array}\right)-\sum_{q}\left(\begin{array}{c}
m_{q} \\
2
\end{array}\right) \quad \text { and } \quad \sum_{q^{\prime}} m_{q^{\prime}} \leq m_{p}
$$

The formula on the left was known by Max Noether; see (4.1). It gives the genus $g$ of the irreducible plane curve $\mathcal{C}$ of degree $d$, where $q$ varies over all singularities on, and infinitely near, $\mathcal{C}$, and $m_{q}$ is the multiplicity at $q$. The formula on the right holds for any point $p$ on any curve $\mathcal{C}$; the points $q^{\prime}$ vary over all of the points in the first neighborhood of $p$. The above formulas permit 9 possible singularities on a rational plane curve of degree 4 :

$\begin{array}{cccc}\begin{array}{c}\text { Classical } \\ \text { name }\end{array} & \begin{array}{c}\text { modern } \\ \text { name }\end{array} & \begin{array}{c}\text { multiplicity } \\ \text { sequence }\end{array} & (m, \delta, s) \\ \text { Node } & A_{1} & (2: 1,1) & (2,1,2) \\ \text { Cusp } & A_{2} & (2: 1) & (2,1,1) \\ \text { Tacnode } & A_{3} & (2: 2: 1,1) & (2,2,2) \\ \text { Ramphoid Cusp } & A_{4} & (2: 2: 1) & (2,2,1) \\ \text { Oscnode } & A_{5} & (2: 2: 2: 1,1) & (2,3,2) \\ A_{6} \text {-Cusp } & A_{6} & (2: 2: 2: 1) & (2,3,1) \\ \text { Ordinary Triple Point } & D_{4} & (3: 1,1,1) & (3,3,3) \\ \text { Tacnode Cusp } & D_{5} & (3: 1,1) & (3,3,2) \\ \text { Multiplicity 3 Cusp } & E_{6} & (3: 1) & (3,3,1)\end{array}$

The singularity " $A_{6}$ " does not have a consistent classical name and for that reason we have introduced the name " $A_{6}$-cusp". Beware that Namba's terminology is not completely consistent with the terminology used above: he uses "double cusp" (respectively, "ramphoid cusp") for what we call a ramphoid cusp (respectively, 
" $A_{6}$-cusp"). The thirteen possible ways to configure the above singularities on a rational plane quartic are given in (0.3). For each configuration $\%$ from (0.3) define

$$
S_{\%}=\left\{\boldsymbol{g} \in \mathbb{T}_{4} \mid \text { the configuration of singularities on } \mathcal{C}_{\boldsymbol{g}} \text { are described by } \%\right\} \text {. }
$$

Thus, $\mathbb{T}_{4}$ is the disjoint union of $S_{\%}$ as $\%$ roams over the 13 configurations listed in (0.3). We use open and closed subsets in $\mathbb{T}_{4}$ and $\mathbb{B}_{4}$ to separate the subsets $S_{\%}$. We describe the open and closed sets in $\mathbb{T}_{4}$ by making use of partial orders on the set of 13 possible configurations of singularities, exactly as was done in Section 6. In Definition 9.2 we introduce two partial orders: QCP and BQP. The closed sets that we identify in the proof of Proposition 9.3 come from the techniques of Section 6. The closed sets of Lemma 9.4 arise from the techniques of Section 7. The techniques of Sections 6 and 7 are not able to separate

$$
S_{(2: 2: 1),(2: 1,1)} \quad \text { from } \quad S_{(2: 2: 1,1),(2: 1)}
$$

and for that reason we set

$$
T_{(2: 2: 1),(2: 1,1)}^{\mathrm{QCP}}=T_{(2: 2: 1,1),(2: 1)}^{\mathrm{QCP}} .
$$

The sets of (9.1) are separated in Proposition 9.5, which uses the techniques of Section 8. This separation is reflected in the poset BQP.

The main result of the present section is Corollary 9.11 where we exhibit a stratification of $\mathbb{B}_{4}$ in which every curve associated to a given stratum has the same configuration of singularities. The stratification of Corollary 9.11 is based on the poset BQP. A second major result in this section is Theorem 9.12 where we identify a large collection of closed irreducible subsets of $\mathbb{T}_{4}$; these subsets are parameterized by the elements of of the poset QCP. In both Theorems the dimension of each closed irreducible set is given.

The third major result in this section is Theorem 9.6 where we extend Theorem 4.8 in order to describe a pretty parameterization of each rational plane quartic $\mathcal{C}$ as a function of the singularity configuration of $\mathcal{C}$. In addition to being a complete classification of parameterizations, Theorem 9.6 is used extensively in our proof of irreducibility in Proposition 9.7.

Definition 9.2. We define two posets: the "Quartic Configuration Poset" QCP and the "Balanced Quartic Poset" BQP. The elements of QCP are the 13 configurations of $(0.3)$ once $(2: 2: 1),(2: 1,1)$ has been set equal to $(2: 2: 1,1),(2: 1)$. The order in QCP is given in Table 2, where we have drawn $\%^{\prime} \rightarrow \%$ to mean $\%^{\prime} \leq \%$. For each $\% \in \mathrm{QCP}$, define

$$
T_{\%}^{\mathrm{QCP}}=\bigcup_{\left\{\%^{\prime} \in \mathrm{QCP} \mid \%^{\prime} \leq \%\right\}} S_{\%^{\prime}} .
$$




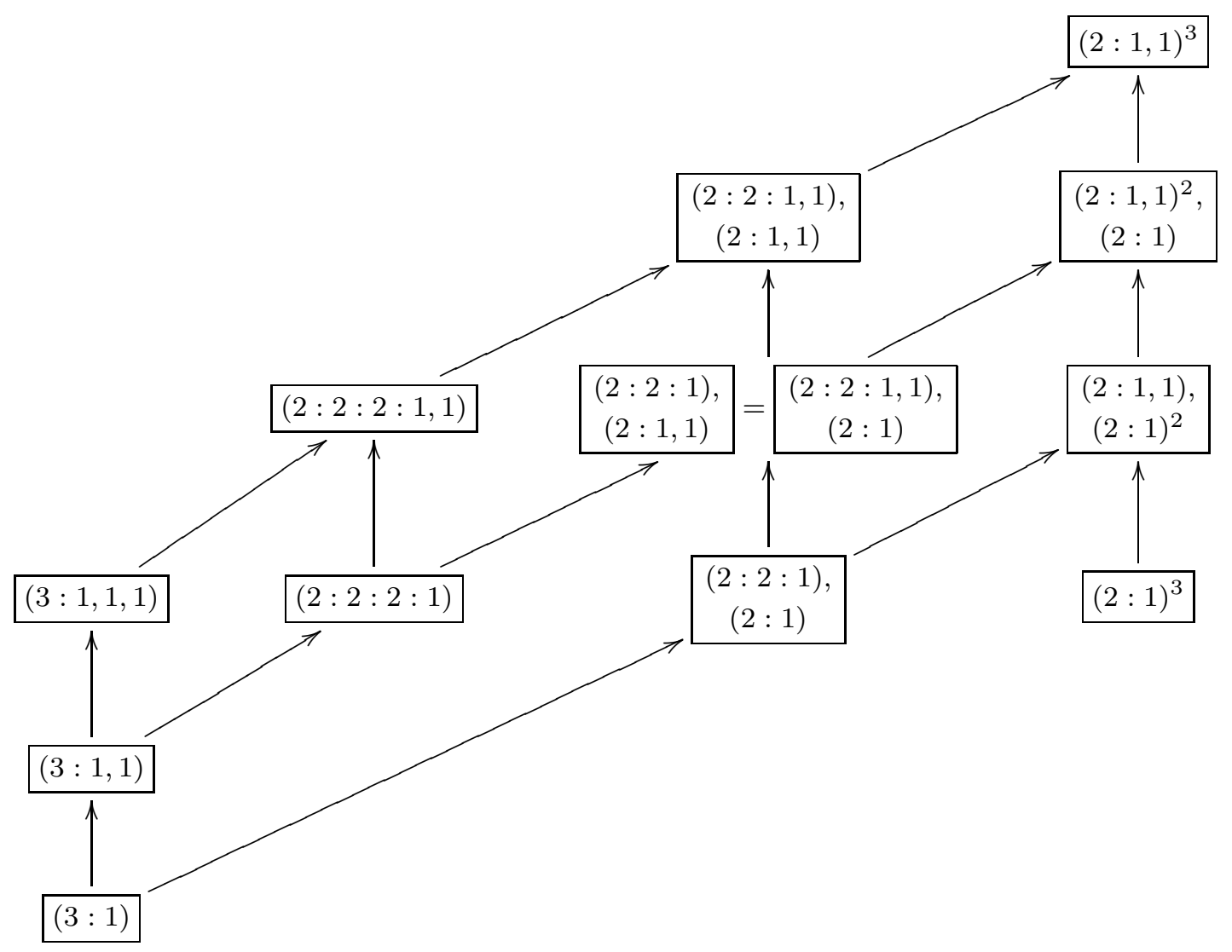

Table 2: The poset QCP.

The elements of $\mathrm{BQP}$ are the 10 balanced singularity configurations for a rational plane quartic. (These are the configurations which involve singularities of multiplicity 2.) The order in BQP is given in Table 3 where we have drawn $\%^{\prime} \rightarrow \%$ in $\mathrm{BQP}$ to mean $\%^{\prime} \leq \%$ in $\mathrm{BQP}$. For each $\%$ in BQP, define

$$
T_{\%}^{\mathrm{BQP}}=\bigcup_{\left\{\%^{\prime} \in \mathrm{BQP} \mid \%^{\prime} \leq \%\right\}} S_{\%} \cdot
$$

Remark. Let $\%$ and $\%^{\prime}$ be singularity configurations $(2: 2: 1),(2: 1,1)$ and $(2: 2: 1,1),(2: 1)$, respectively. Please notice that $S_{\%}$ and $S_{\%^{\prime}}$ are disjoint subsets of $\mathbb{T}_{4}$; but the subsets $T_{\%}^{\mathrm{QCP}}$ and $T_{\%^{\prime}}^{\mathrm{QCP}}$ of $\mathbb{T}_{4}$ are equal.

Proposition 9.3. For each $\% \in \mathrm{QCP}, T_{\%}^{\mathrm{QCP}}$ is a closed subset of $\mathbb{T}_{4}$.

Proof. The subset $T_{(3: 1,1,1)}^{\mathrm{QCP}}$ of $\mathbb{T}_{4}$ is the same as the subset $\mathbb{U B}_{4}$ and this is closed in $\mathbb{T}_{4}$ by Theorem 5.24. The subset $S_{(2: 2: 2: 1,1)} \cup S_{(2: 2: 2: 1)}$ in the present notation 


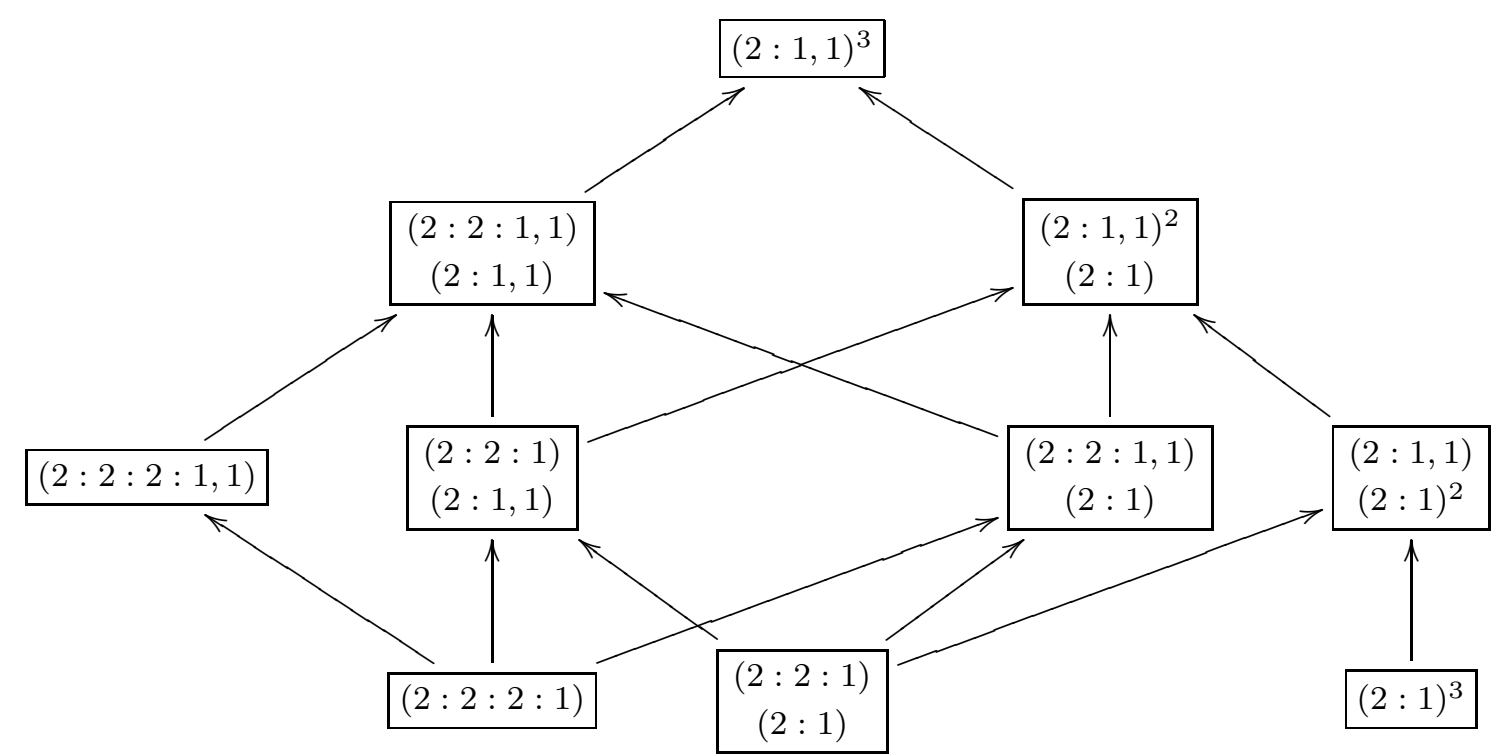

Table 3: The poset BQP.

is the same as the subset $T_{2: 2: 2}$ of $\mathbb{B}_{4}$ in the notation of Section 6. Theorem 6.10 shows that $T_{2: 2: 2}$ is closed in $\mathbb{B}_{4}$; hence,

$$
T_{(2: 2: 2: 1,1)}^{\mathrm{QCP}}=\mathbb{U B}_{4} \cup T_{2: 2: 2}
$$

is closed in $\mathbb{T}_{4}$. Repeat this argument for the closed subsets $T_{2: 2,2}$ and $T_{2,2,2}$ of $\mathbb{B}_{4}$ to conclude that $T_{\%}^{\mathrm{QCP}}$ is closed in $\mathbb{T}_{4}$ for $\%=(2: 2: 1,1),(2: 1,1)$ and for $\%=(2: 1,1)^{3}$. We have shown that $T_{\%}^{\mathrm{QCP}}$ is closed in $\mathbb{T}_{4}$ for each $\%$ across the top row of Table 2 .

Lemma 9.4 shows that $T_{\%}^{\mathrm{QCP}}$ is a closed subset of $\mathbb{T}_{4}$ for $\%$ equal to

$$
(2: 1,1)^{2},(2: 1), \quad(2: 1,1),(2: 1)^{2}, \quad \text { or } \quad(2: 1)^{3} .
$$

That is; $T_{\%}^{\mathrm{QCP}}$ is closed in $\mathbb{T}_{4}$ for each $\%$ down the right hand column of Table 2 .

If $\%$ is an element of QCP and \% does not live in the top row or the right hand column of Table 2 , then $T_{\%}^{\mathrm{QCP}}$ is the intersection of two closed subsets of $\mathbb{T}_{4}$.

Lemma 9.4. Take $\boldsymbol{R}, \boldsymbol{S}$, and $G_{j}$ from Conventions 5.15. Let $N$ be the matrix

$$
N=\left[\begin{array}{lll}
\frac{\partial G_{1}}{\partial x} & \frac{\partial G_{2}}{\partial x} & \frac{\partial G_{3}}{\partial x} \\
\frac{\partial G_{1}}{\partial y} & \frac{\partial G_{2}}{\partial y} & \frac{\partial G_{3}}{\partial y}
\end{array}\right]
$$


and $S$ be the ring $\boldsymbol{S} / I_{2}(N)$. Let $\boldsymbol{R} \rightarrow S$ be the natural map induced by the inclusion $\boldsymbol{R} \subseteq \boldsymbol{S}$. Recall the language of Convention (4) from 5.15. The following statements hold.

(1) The set $X(S ;=1, \geq 1) \cap \mathbb{T}_{4}=T_{(2: 1,1)^{2},(2: 1)}^{\mathrm{QCP}}$ is a closed subset of $\mathbb{T}_{4}$.

(2) The set $X(S ;=1, \geq 2) \cap \mathbb{T}_{4}=T_{(2: 1,1),(2: 1)^{2}}^{\mathrm{QCP}}$ is a closed subset of $\mathbb{T}_{4}$.

(3) The set $X(S ;=1, \geq 3) \cap \mathbb{T}_{4}=T_{(2: 1)^{3}}^{\mathrm{QCP}}$ is a closed subset of $\mathbb{T}_{4}$.

Proof. Observe first that if $\boldsymbol{g} \in \mathbb{A}_{d}$, then $S_{\lambda_{\boldsymbol{g}}}$ is equal to the $\operatorname{ring} B /\left(\operatorname{Jac}\left(B / \boldsymbol{k}\left[I_{\boldsymbol{g}}\right]\right)\right)$. If $\boldsymbol{g} \in \mathbb{T}_{4}$, then ht $I_{\boldsymbol{g}}=2$ and this guarantees that $\operatorname{Jac}\left(B / \boldsymbol{k}\left[I_{\boldsymbol{g}}\right]\right)$ is not the zero ideal. It follows that the dimension of $S_{\lambda_{g}}$ is either 0 or 1 . Thus, $X(S ; \geq 1) \cap \mathbb{T}_{4}$ is equal to $X(S ;=1) \cap \mathbb{T}_{4}$. According to Theorem $5.13, X(S ; \geq 1) \cap \mathbb{T}_{4}$ is closed in $\mathbb{T}_{4}$. It follows that $X(S ;=1) \cap \mathbb{T}_{4}$ is closed in $\mathbb{T}_{4}$. Theorem 5.13 also shows that $X(S ;=1, \geq i) \cap \mathbb{T}_{4}$ is closed in $X(S ;=1) \cap \mathbb{T}_{4}$; hence, $X(S ;=1, \geq i) \cap \mathbb{T}_{4}$ is closed in $\mathbb{T}_{4}$. Theorem 7.4 shows

$$
X(S ; \geq 1, \geq i) \cap \mathbb{T}_{d}=\left\{\boldsymbol{g} \in \mathbb{T}_{d} \mid \sum_{p \in \operatorname{Sing} \mathcal{C}_{\boldsymbol{g}}} m_{p}-s_{p} \geq i\right\}
$$

It is now easy easy to compute $\sum m_{p}-s_{p}$ for each $\mathcal{C}$ in $S_{\%}$ for each $\%$ from $(0.3)$. The order in QCP was chosen in order to make

$$
\begin{aligned}
& X(S ;=1, \geq 1) \cap \mathbb{T}_{4}=T_{(2: 1,1)^{2},(2: 1)}^{\mathrm{QCP}}, \\
& X(S ;=1, \geq 2) \cap \mathbb{T}_{4}=T_{(2: 1,1),(2: 1)^{2}}^{\mathrm{QCP}}, \text { and } \\
& X(S ;=1, \geq 3) \cap \mathbb{T}_{4}=T_{(2: 1)^{3}}^{\mathrm{QCP}}
\end{aligned}
$$

and this completes the proof.

Proposition 9.5. For each $\% \in \mathrm{BQP}, T_{\%}^{\mathrm{BQP}}$ is a closed subset of $\mathbb{B}_{4}$.

Proof. Let $\%$ be an element of BQP. Assume first that \% is not equal to

$$
(2: 2: 1),(2: 1,1) \text { or }(2: 2: 1,1),(2: 1) \text {. }
$$

In this case $T_{\%}^{\mathrm{QCP}} \cap \mathbb{B}_{4}=T_{\%}^{\mathrm{BQP}}$. We proved in Proposition 9.3 that $T_{\%}^{\mathrm{QCP}}$ is closed in $\mathbb{T}_{4}$. It follows that $T_{\%}^{\mathrm{QCP}} \cap \mathbb{B}_{4}$ is closed in the subspace $\mathbb{B}_{4}$ of $\mathbb{T}_{4}$.

We now prove that the sets $T_{(2: 2: 1),(2: 1,1)}^{\mathrm{BQP}}$ and $T_{(2: 2: 1,1),(2: 1)}^{\mathrm{BQP}}$ are closed in $\mathbb{B}_{4}$. In Table 4, we recorded the poset $\mathrm{QCP} \cap \mathbb{B}_{4}$ together with the form of the factorization 


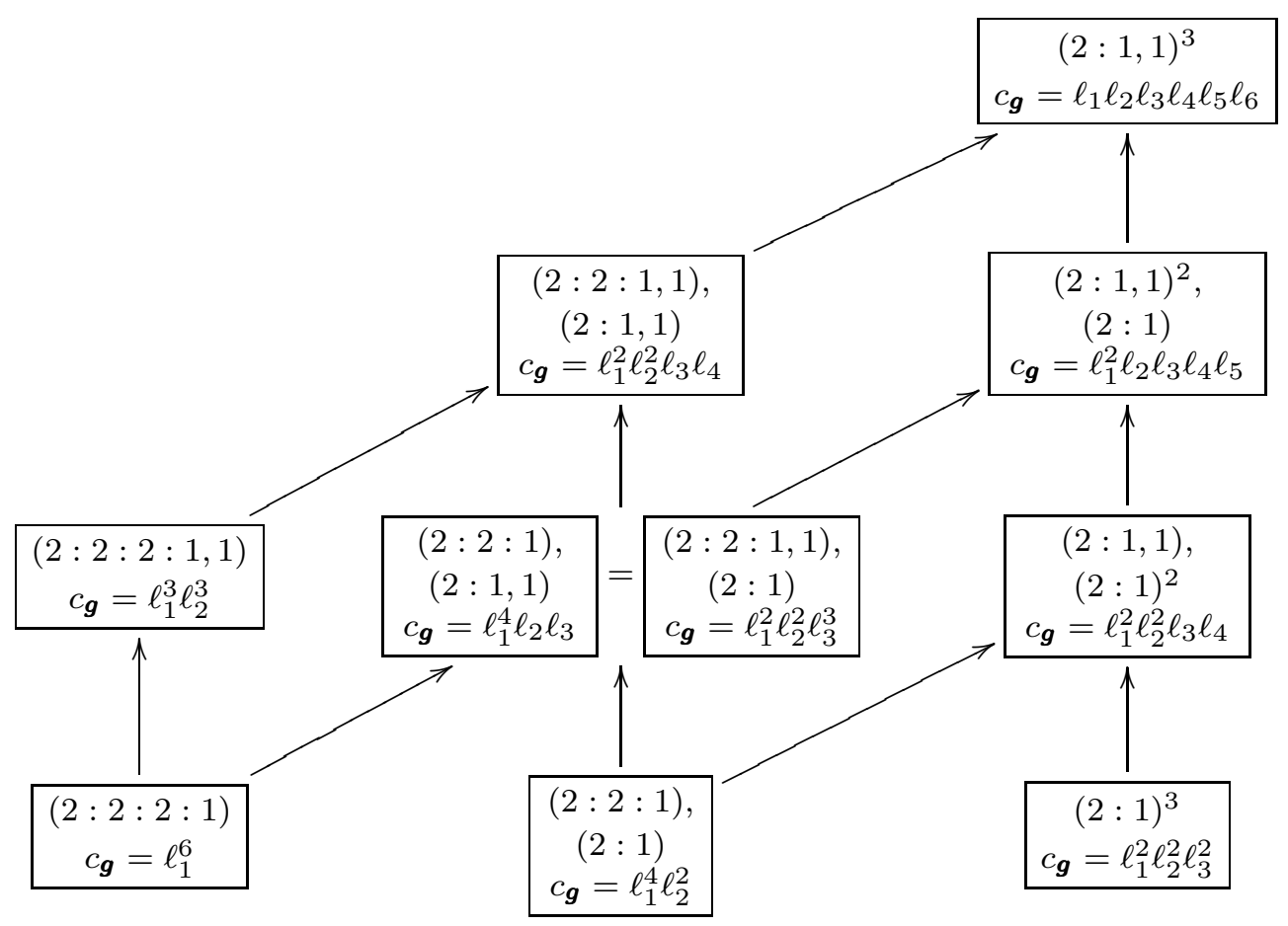

Table 4: The poset $\mathrm{QCP} \cap \mathbb{B}_{4}$ with $c_{\boldsymbol{g}}$.

of the polynomial $c_{\boldsymbol{g}}$ for each $\boldsymbol{g} \in S_{\%}$ for each $\%$ in $\mathrm{QCP} \cap \mathbb{B}_{4}$. The factorizations of $c_{\boldsymbol{g}}$ were calculated in Theorem 8.12. Recall the sets

$$
X_{8.18}=\left\{\boldsymbol{g} \in \mathbb{B}_{d} \mid c_{\boldsymbol{g}}=\prod \ell_{j}^{t_{j}} \text { with all } t_{j} \geq 2\right\}
$$

and

$$
X_{4,8.20}=\left\{\boldsymbol{g} \in \mathbb{B}_{d} \mid c_{\boldsymbol{g}}=\prod \ell_{j}^{t_{j}} \text { with at least one } t_{j} \geq 4\right\}
$$

from Theorems 8.18 and 8.20. A quick look at Table 4 shows that

$X_{8.18} \cap\left(T_{(2: 2: 1,1),(2: 1)}^{\mathrm{QCP}} \cap \mathbb{B}_{4}\right)=S_{(2: 2: 1,1),(2: 1)} \cup S_{(2: 2: 1),(2: 1)} \cup S_{(2: 2: 2: 1)}=T_{(2: 2: 1,1),(2: 1)}^{\mathrm{BQP}}$

and

$X_{4,8.20} \cap\left(T_{(2: 2: 1),(2: 1,1)}^{\mathrm{QCP}} \cap \mathbb{B}_{4}\right)=S_{(2: 2: 1),(2: 1,1)} \cup S_{(2: 2: 1),(2: 1)} \cup S_{(2: 2: 2: 1)}=T_{(2: 2: 1),(2: 1,1)}^{\mathrm{BQP}}$.

Theorems 8.18 and 8.20 show that $X_{8.18}$ and $X_{4,8.20}$ are closed subsets of $\mathbb{B}_{4}$ and Proposition 9.3 shows that

$$
T_{(2: 2: 1),(2: 1,1)}^{\mathrm{QCP}} \cap \mathbb{B}_{4}=T_{(2: 2: 1,1),(2: 1)}^{\mathrm{QCP}} \cap \mathbb{B}_{4}
$$


is a closed subset of $\mathbb{B}_{4}$. We conclude that $T_{(2: 2: 1,1),(2: 1)}^{\mathrm{BQP}}$ and $T_{(2: 2: 1),(2: 1,1)}^{\mathrm{BQP}}$ are closed subsets of $\mathbb{B}_{4}$.

Theorem 9.6 gives the canonical form for a parameterization of some representative of the right equivalence class of each rational plane quartic $\mathcal{C}$. If $\mathcal{C}$ has any singularities of multiplicity 2 , then all of its singularities have multiplicity 2 and the techniques of Section 4 apply to all of the singularities. For this reason, the description of the Hilbert-Burch matrix in the present result is much more detailed than the description of Theorem 4.8. In addition to being a very pretty classification, Theorem 9.6 is the starting point in the proof of Proposition 9.7 about the irreducibility of many subsets of $\mathbb{T}_{4}$. (We first used the phrase "signed maximal order minors" in Remark 4.4 (2).)

Theorem 9.6. Let $\mathcal{C}$ be a rational plane quartic over an algebraically closed field. Then there exists a linear automorphism $\Lambda$ of $\mathbb{P}^{2}$ so that $\Lambda \mathcal{C}$ is parameterized by the signed maximal order minors of $\varphi_{\%}$, where $\varphi_{\%}$ is given below. In each case, the entries of $\varphi_{\%}$ must be chosen so that ht $I_{2}\left(\varphi_{\%}\right)=2$ and $\mu\left(I_{1}\left(\varphi_{\%}\right)\right) \geq 3$. We write $\ell_{i}$ for linear form, $Q_{i}$ for quadratic form, and $C_{i}$ for cubic form.

(1) If the singularity configuration of $\mathcal{C}$ is given by $\%$ is equal to one of

$$
(2: 1,1)^{3}, \quad(2: 1,1)^{2},(2: 1), \quad(2: 1,1),(2: 1)^{2}, \quad \text { or } \quad(2: 1)^{3}
$$

then

$$
\varphi_{\%}=\left[\begin{array}{cc}
Q_{1} & Q_{1} \\
Q_{2} & 0 \\
0 & Q_{3}
\end{array}\right]
$$

(a) If $\%=(2: 1,1)^{3}$, then each $Q_{i}$ is the product of 2 non-associate linear forms in $B$. The singularities of $\Lambda \mathcal{C}$ are $[0: 0: 1],[0: 1: 0]$, and $[1: 0: 0]$. Each singularity is an ordinary node.

(b) If $\%=(2: 1,1)^{2},(2: 1)$, then each of the polynomials $Q_{1}$ and $Q_{2}$ is the product of 2 non-associate linear forms in $B$ and $Q_{3}$ is a perfect square. The singularities of $\Lambda \mathcal{C}$ are $[0: 0: 1],[0: 1: 0]$, and $[1: 0: 0]$. The singularities $[1: 0: 0],[0: 1: 0]$ are ordinary nodes; and $[0: 0: 1]$ is a cusp.

(c) If $\%=(2: 1,1),(2: 1)^{2}$, then $Q_{1}$ is the product of 2 non-associate linear forms in $B$ and each of the polynomials $Q_{2}, Q_{3}$ is a perfect square. The singularities of $\Lambda \mathcal{C}$ are $[0: 0: 1],[0: 1: 0]$, and $[1: 0: 0]$. The singularity $[1: 0: 0]$ is an ordinary node; and $[0: 1: 0]$ and $[0: 0: 1]$ are cusps.

(d) If $\%=(2: 1)^{3}$, then all three polynomials $Q_{1}, Q_{2}$, and $Q_{3}$ are perfect squares. The singularities of $\Lambda \mathcal{C}$ are $[0: 0: 1],[0: 1: 0]$, and $[1: 0: 0]$. Each singularity is a cusp. 
(2) If the singularity configuration of $\mathcal{C}$ is given by $\%$ is equal to

$$
\begin{array}{ll}
(2: 2: 1,1),(2: 1,1), & (2: 2: 1,1),(2: 1), \\
(2: 2: 1),(2: 1,1), \text { or } \quad(2: 2: 1),(2: 1),
\end{array}
$$

then

$$
\varphi_{\%}=\left[\begin{array}{cc}
Q_{1} & 0 \\
Q_{2} & Q_{3} \\
0 & Q_{2}
\end{array}\right]
$$

(a) If $\%=(2: 2: 1,1),(2: 1,1)$, then $Q_{1}$ and $Q_{2}$ are the product of 2 nonassociate linear forms in $B$. The singularities of $\Lambda \mathcal{C}$ are $[0: 0: 1]$, and $[1: 0: 0]$. The singularity $[1: 0: 0]$ is an ordinary node and $[0: 0: 1]$ is a tacnode.

(b) If $\%=(2: 2: 1,1),(2: 1)$, then $Q_{1}$ is a perfect square and $Q_{2}$ is the product of 2 non-associate linear forms in $B$. The singularities of $\Lambda \mathcal{C}$ are $[0: 0: 1]$, and $[1: 0: 0]$. The singularity $[1: 0: 0]$ is an cusp and $[0: 0: 1]$ is a tacnode.

(c) If $\%=(2: 2: 1),(2: 1,1)$, then $Q_{2}$ is a perfect square and $Q_{1}$ is the product of 2 non-associate linear forms in $B$. The singularities of $\Lambda \mathcal{C}$ are $[0: 0: 1]$, and $[1: 0: 0]$. The singularity $[1: 0: 0]$ is an ordinary node and $[0: 0: 1]$ is a ramphoid cusp.

(d) If $\%=(2: 2: 1),(2: 1)$, then $Q_{1}$ and $Q_{2}$ are both perfect squares. The singularities of $\Lambda \mathcal{C}$ are $[0: 0: 1]$, and $[1: 0: 0]$. The singularity $[1: 0: 0]$ is a cusp and $[0: 0: 1]$ is a ramphoid cusp.

(3) If the singularity configuration of $\mathcal{C}$ is given by $\%$ is equal to

$$
(2: 2: 2: 1,1) \quad \text { or } \quad(2: 2: 2: 1)
$$

then

$$
\varphi_{\%}=\left[\begin{array}{cc}
Q_{1} & Q_{2} \\
Q_{3} & Q_{1} \\
0 & Q_{3}
\end{array}\right] .
$$

(a) If $\%=(2: 2: 2: 1,1)$, then $Q_{3}$ is the product of 2 non-associate linear forms in $B$. The only singularity on $\Lambda \mathcal{C}$ is $[0: 0: 1]$ and this singularity is an oscnode.

(b) If $\%=(2: 2: 2: 1)$, then $Q_{3}$ is a perfect square. The only singularity on $\Lambda \mathcal{C}$ is $[0: 0: 1]$ and this singularity is an $A_{6}$-cusp.

(4) If the singularity configuration of $\mathcal{C}$ is given by $\%$ is equal to

$$
(3: 1,1,1), \quad(3: 1,1), \quad \text { or } \quad(3: 1) \text {, }
$$


then

$$
\varphi_{\%}=\left[\begin{array}{cc}
\ell_{1} & C_{1} \\
\ell_{2} & C_{2} \\
0 & C_{3}
\end{array}\right] .
$$

(a) If $\%=(3: 1,1,1)$, then $C_{3}$ is the product of 3 non-associate linear forms in $B$. The only singularity on $\Lambda \mathcal{C}$ is $[0: 0: 1]$ and this singularity is an ordinary triple point.

(b) If $\%=(3: 1,1)$, then $C_{3}$ has the form $\ell_{3}^{2} \ell_{4}$, where $\ell_{3}$ and $\ell_{4}$ non-associate linear forms from $B$. The only singularity on $\Lambda \mathcal{C}$ is $[0: 0: 1]$ and this singularity is a tacnode cusp.

(c) If $\%=(3: 1)$, then $C_{3}$ is a perfect cube. The only singularity on $\Lambda \mathcal{C}$ is $[0: 0: 1]$ and this singularity is a multiplicity three cusp.

Proof. The basic form of $\varphi_{\%}$ is described by Theorem 4.8 when $\mathcal{C}$ has a multiplicity 2 singularity and by Corollary 1.9 when $\mathcal{C}$ has a multiplicity 3 singularity. The information about the number of non-associate linear factors is an immediate consequence of Theorem 1.8.

\section{Proposition 9.7.}

(a) The subsets $T_{(3: 1)}^{\mathrm{QCP}}, T_{(3: 1,1)}^{\mathrm{QCP}}, T_{(3: 1,1,1)}^{\mathrm{QCP}}, T_{(2: 1,1)^{3}}^{\mathrm{QCP}}$, and $T_{(2: 1,1)^{2},(2: 1)}^{\mathrm{QCP}}$ of $\mathbb{T}_{4}$ are irreducible.

(b) The subset $T_{\%}^{\mathrm{BQP}}$ of $\mathbb{B}_{4}$ is irreducible for all ten $\%$ in the poset $\mathrm{BQP}$.

Proof. The subset $T_{(2: 1,1)^{3}}^{\mathrm{QCP}}$ of $\mathbb{T}_{4}$ is by definition equal to all of $\mathbb{T}_{4}$. Theorem 5.20 shows that $\mathbb{T}_{4}$ is an open subset of the irreducible space $\mathbb{A}_{4}$. It follows that $\mathbb{T}_{4}$ is also irreducible. The subsets $T_{(2: 2: 2: 1,1)}^{\mathrm{BQP}}$ and $T_{(2: 2: 1,1),(2: 1,1)}^{\mathrm{BQP}}$ of $\mathbb{B}_{4}$ are called $S_{2: 2: 2}$ and $S_{2: 2,2}$ in Section 6 . Theorem 6.10 shows that these sets are irreducible. We use the following trick repeatedly throughout the rest of the argument.

(9.8) If $Y$ is an irreducible variety and $\theta: Y \rightarrow \mathbb{A}_{d}$ is a morphism, then $\operatorname{im} \theta \cap \mathbb{T}_{d}$ is an irreducible set.

Proof of (9.8). The set $\mathbb{T}_{d}$ is open in $\mathbb{A}_{d}$; so, $\theta^{-1}\left(\mathbb{T}_{d}\right)$ is an open subset of the irreducible set $Y$. It follows that $Y \cap \theta^{-1}\left(\mathbb{T}_{d}\right)$ is an irreducible set. The image of the irreducible set $Y \cap \theta^{-1}\left(\mathbb{T}_{d}\right)$ under the morphism is necessarily irreducible; and this image is equal to $\theta(Y) \cap \mathbb{T}_{d}$. The proof of (9.8) is complete.

We consider a situation which is very similar to Definition 4.3 and Remarks 4.4. Let $\mathcal{G}$ be the multiplicative group of matrices

$$
\mathcal{G}=\left\{\left[\begin{array}{cc}
u_{1} & Q \\
0 & u_{2}
\end{array}\right] \mid u_{1}, u_{2} \in \boldsymbol{k}^{*}, Q \in B_{2}\right\}
$$


and $G^{\prime}$ be the group $\mathrm{GL}_{3}(\boldsymbol{k}) \times \mathcal{G}$. The group $G^{\prime}$ acts on the space of matrices

$$
\mathbb{H}^{\prime}=\left\{\left[\begin{array}{ll}
\ell_{1} & C_{1} \\
\ell_{2} & C_{2} \\
\ell_{3} & C_{3}
\end{array}\right] \mid \ell_{i} \in B_{1} \text { and } C_{i} \in B_{3}\right\}
$$

by $(\chi, \xi) \cdot \varphi=\chi \varphi \xi^{-1}$ for $\chi \in \mathrm{GL}_{3}(\boldsymbol{k}), \xi \in \mathcal{G}$, and $\varphi \in \mathbb{H}^{\prime}$. Let $\Phi$ be the morphism which is defined in (4.5).

Consider the morphism $\theta: G^{\prime} \times B_{1}^{3} \times B_{3}^{2} \rightarrow \mathbb{A}_{4}$, which is given by

$$
\theta\left(g, \ell_{1}, \ell_{2}, \ell_{3}, C_{1}, C_{2}\right)=\Phi\left(g \cdot\left[\begin{array}{cc}
\ell_{1} & C_{1} \\
\ell_{2} & C_{2} \\
0 & \ell_{3}^{3}
\end{array}\right]\right)
$$

Theorem 9.6 shows that $\operatorname{im} \theta \cap \mathbb{T}_{4}=T_{(3: 1)}^{\mathrm{QCP}}$. The domain of $\theta$ is an irreducible variety; so (9.8) shows that $T_{(3: 1)}^{\mathrm{QCP}}$ is an irreducible subset of $\mathbb{T}_{4}$. Let

$$
\Delta=\left\{C \in B_{3} \mid \text { the discriminant of } C \text { is zero }\right\} .
$$

Define $\theta: G^{\prime} \times B_{1}^{2} \times B_{3}^{2} \times \Delta \rightarrow \mathbb{A}_{4}$ by

$$
\theta\left(g, \ell_{1}, \ell_{2}, C_{1}, C_{2}, C\right)=\Phi\left(g \cdot\left[\begin{array}{cc}
\ell_{1} & C_{1} \\
\ell_{2} & C_{2} \\
0 & C
\end{array}\right]\right)
$$

The domain of $\theta$ is an irreducible variety and Theorem 9.6 shows that $\operatorname{im} \theta \cap \mathbb{T}_{4}$ is $T_{(3: 1,1)}^{\mathrm{QCP}}$. It follows from $(9.8)$ that $T_{(3: 1,1)}^{\mathrm{QCP}}$ is an irreducible subset of $\mathbb{T}_{4}$. We abbreviate the argument. Consider the morphisms

$$
\begin{array}{lll}
\theta_{1}: G^{\prime} \times B_{1}^{2} \times B_{3}^{3} \rightarrow \mathbb{A}_{4}, & \theta_{2}: G \times B_{1} \times B_{2}^{3} \rightarrow \mathbb{A}_{4}, & \theta_{3}: G \times B_{1} \times B_{2}^{2} \rightarrow \mathbb{A}_{4}, \\
\theta_{4}: G \times B_{1}^{2} \times B_{2} \rightarrow \mathbb{A}_{4}, & \theta_{5}: G \times B_{1}^{3} \rightarrow \mathbb{A}_{4}, & \theta_{6}: G \times B_{1}^{2} \times B_{2}^{2} \rightarrow \mathbb{A}_{4}, \\
\theta_{7}: G \times B_{1} \times B_{2}^{4} \rightarrow \mathbb{A}_{4}, & \theta_{8}: G \times \mathbb{A}^{3} \times B_{1} \times B_{2}^{2} \rightarrow \mathbb{A}_{4} &
\end{array}
$$

which are given by

$$
\begin{gathered}
\theta_{1}\left(g, \ell_{1}, \ell_{2}, C_{1}, C_{2}, C_{3}\right)=\Phi\left(g \cdot\left[\begin{array}{cc}
\ell_{1} & C_{1} \\
\ell_{2} & C_{2} \\
0 & C_{3}
\end{array}\right]\right), \\
\theta_{2}\left(g, \ell, Q_{1}, Q_{2}, Q_{3}\right)=\Phi\left(g \cdot\left[\begin{array}{cc}
Q_{1} & Q_{2} \\
\ell^{2} & Q_{3} \\
0 & \ell^{2}
\end{array}\right]\right),
\end{gathered}
$$




$$
\begin{gathered}
\theta_{3}\left(g, \ell, Q_{1}, Q_{2}\right)=\Phi\left(g \cdot\left[\begin{array}{cc}
Q_{1} & Q_{2} \\
\ell^{2} & Q_{1} \\
0 & \ell^{2}
\end{array}\right]\right), \\
\theta_{4}\left(g, \ell_{1}, \ell_{2}, Q\right)=\Phi\left(g \cdot\left[\begin{array}{cc}
\ell_{1}^{2} & 0 \\
\ell_{2}^{2} & Q \\
0 & \ell_{2}^{2}
\end{array}\right]\right), \\
\theta_{5}\left(g, \ell_{1}, \ell_{2}, \ell_{3}\right)=\Phi\left(g \cdot\left[\begin{array}{cc}
\ell_{1}^{2} & \ell_{1}^{2} \\
\ell_{2}^{2} & 0 \\
0 & \ell_{3}^{2}
\end{array}\right]\right), \\
\theta_{6}\left(g, \ell_{1}, \ell_{2}, Q_{3}, Q_{4}\right)=\Phi\left(g \cdot\left[\begin{array}{cc}
Q_{3} & Q_{4} \\
\ell_{2}^{2} & 0 \\
0 & \ell_{1}^{2}
\end{array}\right]\right), \\
\theta_{7}\left(g, \ell_{1}, Q_{1}, Q_{2}, Q_{3}, Q_{4}\right)=\Phi\left(g \cdot\left[\begin{array}{cc}
Q_{1} & Q_{2} \\
Q_{3} & Q_{4} \\
0 & \ell_{1}^{2}
\end{array}\right]\right) \text { and } \\
\theta_{8}\left(g, a, b, c, \ell, Q_{1}, Q_{2}\right)=\Phi\left(g\left[\begin{array}{cc}
Q_{1} & Q_{2} \\
a \ell^{2} & Q_{1} \\
b \ell^{2} & c \ell^{2}
\end{array}\right]\right) .
\end{gathered}
$$

The domain of each of these morphisms is irreducible. Theorem 9.6 shows that

$$
\operatorname{im} \theta_{i} \cap \mathbb{T}_{4}= \begin{cases}T_{(3: 1,1,1)}^{\mathrm{QCP}} & \text { if } i=1 \\ T_{(2: 2: 1),(2: 1,1)}^{\mathrm{BQP}} & \text { if } i=2 \\ T_{(2: 2: 2: 1)}^{\mathrm{BQP}} & \text { if } i=3 \\ T_{(2: 2: 1),(2: 1)}^{\mathrm{BQP}} & \text { if } i=4 \\ T_{(2: 1)^{3}}^{\mathrm{BQP}} & \text { if } i=5 \\ T_{(2: 1,1),(2: 1)^{2}}^{\mathrm{BQP}} & \text { if } i=6 \\ T_{(2: 1,1)^{2},(2: 1)}^{\mathrm{BQP}} & \text { if } i=7 \\ T_{(2: 2: 1,1),(2: 1)}^{\mathrm{BQP}} & \text { if } i=8 .\end{cases}
$$

We offer some details which are involved in the proof of (9.9). First we show that

$$
\operatorname{im} \theta_{6} \cap \mathbb{T}_{4} \subseteq T_{(2: 1,1),(2: 1)^{2}}^{\mathrm{QCP}} \cap \mathbb{B}_{4} .
$$


Let

$$
\varphi=\left[\begin{array}{cc}
Q_{3} & Q_{4} \\
\ell_{2}^{2} & 0 \\
0 & \ell_{1}^{2}
\end{array}\right]
$$

with $\Phi(\varphi) \in \mathbb{T}_{4}$. There are two cases. Either $Q_{3} \in<\ell_{1}^{2}, \ell_{2}^{2}>$ or $Q_{3} \notin<\ell_{1}^{2}, \ell_{2}^{2}>$. In the first case, there is an element $g$ of $G$ with

$$
g \varphi=\left[\begin{array}{cc}
\ell_{2}^{2} & 0 \\
\ell_{1}^{2} & Q_{4} \\
0 & \ell_{1}^{2}
\end{array}\right]
$$

hence, $\Phi(\varphi) \in S_{(2: 2: 1),(2: 1)}$. In the second case, $\ell_{1}^{2}, \ell_{2}^{2}, Q_{3}$ is a basis for $B_{2}$ and $Q_{4}=\alpha_{1} \ell_{1}^{2}+\alpha_{2} \ell_{2}^{2}+\alpha_{3} Q_{3}$, for some constants $\alpha_{1}, \alpha_{2}, \alpha_{3}$. Once again there are two cases: either $\alpha_{3}=0$ or $\alpha_{3} \neq 0$. If $\alpha_{3}=0$, then there exists $g \in G$ with

$$
g \varphi=\left[\begin{array}{cc}
\ell_{1}^{2} & 0 \\
\ell_{2}^{2} & Q_{3} \\
0 & \ell_{2}^{2}
\end{array}\right]
$$

hence, $\Phi(\varphi) \in S_{(2: 2: 1),(2: 1)}$. If $\alpha_{3} \neq 0$, then there exists $g \in G$ and $Q_{3}^{\prime} \in B_{2}$ such that

$$
g \varphi=\left[\begin{array}{cc}
Q_{3}^{\prime} & Q_{3}^{\prime} \\
\ell_{2}^{2} & 0 \\
0 & \ell_{1}^{2}
\end{array}\right]
$$

hence, $\Phi(\varphi) \in S_{(2: 1,1),(2: 1)^{2}} \cup S_{(2: 1)^{3}}$.

It is obvious that $T_{(2,1,1)^{2},(2: 1)}^{\mathrm{QCP}} \cap \mathbb{B}_{4} \subseteq \operatorname{im} \theta_{7} \cap \mathbb{T}_{4}$. We show the converse. Suppose that $\boldsymbol{g} \in \operatorname{im} \theta_{7} \cap \mathbb{T}_{4}$. It is clear that $\boldsymbol{g} \in \mathbb{B}_{4}$. Also, the curve $\mathcal{C}_{\boldsymbol{g}}$ which is parameterized by $\boldsymbol{g}$ has at least one singularity with exactly one branch. A quick look at Table 2 shows that $\boldsymbol{g} \in T_{(2,1,1)^{2},(2: 1)}^{\mathrm{QCP}}$.

We show $\operatorname{im} \theta_{8} \cap \mathbb{T}_{4}=T_{(2: 2: 1,1),(2: 1)}^{\mathrm{BQP}}$. We first prove the inclusion " $\supseteq$ ". Set $a=c=0, b=1$, and $g=1$ to get

$$
\Phi\left(\left[\begin{array}{cc}
Q_{1} & Q_{2} \\
0 & Q_{1} \\
\ell^{2} & 0
\end{array}\right]\right)
$$

Thus, $S_{(2: 2: 1,1),(2: 1)} \cup S_{(2: 2: 1),(2: 1)}$ is contained in $\operatorname{im} \theta_{8} \cap \mathbb{T}_{4}$. Set $a=c=1, b=0$, and $g=1$ to get

$$
\Phi\left(\left[\begin{array}{cc}
Q_{1} & Q_{2} \\
\ell^{2} & Q_{1} \\
0 & \ell^{2}
\end{array}\right]\right)
$$


Thus, $S_{(2: 2: 2: 1)}$ is contained in $\operatorname{im} \theta_{8} \cap \mathbb{T}_{4}$. The set $T_{(2: 2: 1,1),(2: 1)}^{\mathrm{BQP}}$ is equal

$$
S_{(2: 2: 1,1),(2: 1)} \cup S_{(2: 2: 1),(2: 1)} \cup S_{(2: 2: 2: 1)} .
$$

We have shown that $\operatorname{im} \theta_{8} \cap \mathbb{T}_{4} \supseteq T_{(2: 2: 1,1),(2: 1)}^{\mathrm{BQP}}$. Now we prove the direction " $\subseteq$ ". Let

$$
\varphi=\left[\begin{array}{cc}
Q_{1} & Q_{2} \\
a \ell^{2} & Q_{1} \\
b \ell^{2} & c \ell^{2}
\end{array}\right]
$$

Assume that $\Phi(\varphi) \in \mathbb{T}_{4}$. There are two cases: either $b \neq 0$ or $b=0$. If $b \neq 0$, then $\varphi$ may be quickly transformed into

$$
\left[\begin{array}{cc}
Q_{1} & Q_{2}-\frac{c}{b} Q_{1} \\
a \ell^{2} & Q_{1}-\frac{a c}{b} \ell^{2} \\
b \ell^{2} & 0
\end{array}\right]
$$

thus, $\varphi$ may be transformed into

$$
\left[\begin{array}{cc}
Q_{1}-\frac{a c}{b} \ell^{2} & Q_{2}-\frac{c}{b} Q_{1} \\
0 & Q_{1}-\frac{a c}{b} \ell^{2} \\
\ell^{2} & 0
\end{array}\right] .
$$

We see that $\Phi(\varphi)$ is in $S_{(2: 2: 1,1),(2: 1)} \cup S_{(2: 2: 1),(2: 1)}$. If $b=0$, then the hypothesis ht $\Phi(\varphi)=2$ forces ac to be non-zero. One may transform $\varphi$ into

$$
\left[\begin{array}{cc}
Q_{1} & a Q_{2} \\
\ell^{2} & Q_{1} \\
0 & \ell^{2}
\end{array}\right]
$$

We see that $\Phi(\varphi)$ is in $S_{(2: 2: 2: 1)}$.

Now that (9.9) is established, we apply (9.8) to see that most of the subsets $T_{\%}^{\mathrm{QCP}}$ from (a) and all of the subsets $T_{\%}^{\mathrm{BQP}}$ from (b) are irreducible. It remains to show that $T_{(2: 1,1)^{2},(2: 1)}^{\mathrm{QCP}}$ is irreducible. Consider the morphism

$$
\theta: B_{1} \times B_{2}^{2} \times B_{4} \times \mathrm{GL}_{3}(\boldsymbol{k}) \rightarrow \mathbb{A}_{4}
$$

which is given by

$$
\theta\left(\ell, Q_{1}, Q_{2}, F, \chi\right)=\left(\ell^{2} Q_{1}, \ell^{2} Q_{2}, F\right) \chi .
$$

In light of (9.8) it suffices to show that $\operatorname{im} \theta \cap \mathbb{T}_{4}=T_{(2: 1,1)^{2},(2: 1)}^{\mathrm{QCP}}$. The inclusion " $\supseteq$ " may be read from Theorem 9.6. To prove " $\subseteq$ ", we recall from Lemma 9.4 that

$$
T_{(2: 1,1)^{2},(2: 1)}^{\mathrm{QCP}}=\left\{g \in \mathbb{T}_{4} \mid \operatorname{deg} \operatorname{gcd} I_{2}\left(\left.N\right|_{\lambda_{\boldsymbol{g}}}\right) \geq 1\right\}
$$


where

$$
\left.N\right|_{\lambda_{\boldsymbol{g}}}=\left[\begin{array}{lll}
\frac{\partial g_{1}}{\partial x} & \frac{\partial g_{2}}{\partial x} & \frac{\partial g_{3}}{\partial x} \\
\frac{\partial g_{1}}{\partial y} & \frac{\partial g_{2}}{\partial y} & \frac{\partial g_{3}}{\partial y}
\end{array}\right] .
$$

One can quickly check that if $\boldsymbol{g}=\left(x^{2} Q_{1}, x^{2} Q_{2}, F\right)$, then $x$ divides gcd $I_{2}\left(\left.N\right|_{\lambda_{\boldsymbol{g}}}\right)$.

In Corollary 9.11 we exhibit a stratification of $\mathbb{B}_{4}$ where every curve associated to a given stratum has the same configuration of singularities; this is the main result in the present section. The hard work in the proof of this result is carried out in the proof of Theorem 9.10.

Theorem 9.10. If $\%$ is in $\mathrm{BQP}$, then $T_{\%}^{\mathrm{BQP}}$ is a closed irreducible subset of $\mathbb{B}_{4}$ and the dimension of $T_{\%}^{\mathrm{BQP}}$ is given in the following table:

$$
\begin{aligned}
& \operatorname{dim} T_{\%}^{\mathrm{BQP}} \quad \% \\
& 15 \quad(2: 1,1)^{3} \\
& 14 \quad(2: 2: 1,1)(2: 1,1) ; \quad(2: 1,1)^{2},(2: 1) \\
& 13 \quad(2: 2: 2: 1,1) ; \quad(2: 2: 1),(2: 1,1) ; \quad(2: 2: 1,1),(2: 1) ; \quad(2: 1,1),(2: 1)^{2} \\
& 12 \quad(2: 2: 2: 1) ; \quad(2: 2: 1),(2: 1) ; \quad(2: 1)^{3} \text {. }
\end{aligned}
$$

Proof. Proposition 9.5 shows that $T_{\%}^{\mathrm{BQP}}$ is closed in $\mathbb{B}_{4}$; Proposition 9.7 shows that $T_{\%}^{\mathrm{BQP}}$ is irreducible; and Proposition 9.16 calculates its dimension.

Corollary 9.11. The poset $\mathrm{BQP}$ gives a stratification of $\mathbb{B}_{4}$. In other words,

(1) $\mathbb{B}_{4}$ is the disjoint union of $\left\{S_{\%} \mid \% \in \mathrm{BQP}\right\}$,

(2) if $\%^{\prime} \leq \%$ in $\mathrm{BQP}$, then $S_{\%^{\prime}}$ is contained in the closure $\overline{S_{\%}}$ of $S_{\%}$ in $\mathbb{B}_{4}$, and

(3) $T_{\%}^{\mathrm{BQP}}$ is the closure of $S_{\%}$ in $\mathbb{B}_{4}$ for all $\%$ in $\mathrm{BQP}$.

Proof. This result follows immediately from Theorem 9.10 using the technique of the proof of Corollary $6.12(\mathrm{~d})$ and Theorem 6.13.

Theorem 9.12 is our best result about closed irreducible subsets of $\mathbb{T}_{4}$.

\section{Theorem 9.12.}

(1) The subsets $S_{(3: 1)}, S_{(2: 2: 2: 1)}, S_{(2: 2: 1),(2: 1)}$, and $S_{(2: 1)^{3}}$ of $\mathbb{T}_{4}$ are irreducible, closed in $\mathbb{T}_{4}$, and have dimension 12 .

(2) The subset $T_{(3: 1,1)}^{\mathrm{QCP}}$ of $\mathbb{T}_{4}$ is irreducible, closed in $\mathbb{T}_{4}$, and has dimension 13 .

(3) The subsets $T_{(3: 1,1,1)}^{\mathrm{QCP}}$ and $T_{(2: 1,1)^{2},(2: 1)}^{\mathrm{QCP}}$ of $\mathbb{T}_{4}$ are irreducible, closed in $\mathbb{T}_{4}$, and have dimension 14.

(4) The set $T_{(2: 1,1)^{3}}^{\mathrm{QCP}}$ is equal to $\mathbb{T}_{4}$. This set is irreducible, closed in $\mathbb{T}_{4}$, and has dimension 15 . 
Proof. The sets $S_{(2: 2: 2: 1)}$ and $S_{(2: 2: 1),(2: 1)}$ are shown to be closed in $\mathbb{T}_{4}$ in Proposition 9.18. The other listed subsets are shown to be closed in $\mathbb{T}_{4}$ in Proposition 9.3. (Recall that $S_{(2: 1)^{3}}=T_{(2: 1)^{3}}^{Q C P}$ and $S_{(3: 1)}=T_{(3: 1)}^{Q C P}$.) All of the sets are shown to be irreducible in Proposition 9.7. The dimensions are calculated in Proposition 9.16.

Observation 9.13. Each subset $S_{\%}$, with \% from (0.3), has dimension at least 12 .

Proof. Recall the group $\Sigma=\mathrm{GL}_{1}(\boldsymbol{k}) \times \mathrm{SL}_{2}(\boldsymbol{k}) \times \mathrm{SL}_{3}(\boldsymbol{k})$ from (0.4). We define an action of $\Sigma$ on $\mathbb{T}_{d}$. If $\left[\begin{array}{ll}a & c \\ b & d\end{array}\right]$ is an element of $\mathrm{SL}_{2}(\boldsymbol{k})$, then $a x+b y, c x+d y$ is a basis for vector space $B_{1}$ of linear forms in the polynomial ring $B=\boldsymbol{k}[x, y]$. If $\sigma=\left(u,\left(\ell_{1}, \ell_{2}\right), \chi\right)$ is an element of $\Sigma$, where the pair of linear forms $\left(\ell_{1}, \ell_{2}\right)$ represents an element of $\mathrm{SL}_{2}(\boldsymbol{k})$, and $\boldsymbol{g}=\left(g_{1}, g_{2}, g_{3}\right)$ is an element of $\mathbb{T}_{d}$, then define

$$
\sigma \cdot \boldsymbol{g}=u\left[\boldsymbol{g}\left(\ell_{1}, \ell_{2}\right)\right] \chi,
$$

where $\boldsymbol{g}\left(\ell_{1}, \ell_{2}\right)$ is the ordered triple

$$
\left(g_{1}\left(\ell_{1}, \ell_{2}\right), g_{2}\left(\ell_{1}, \ell_{2}\right), g_{3}\left(\ell_{1}, \ell_{2}\right)\right),
$$

$\left[\boldsymbol{g}\left(\ell_{1}, \ell_{2}\right)\right] \chi$ is the product of the ordered triple (9.14) with the matrix $\chi$, and $u \boldsymbol{h}$ is $\left(u h_{1}, u h_{2}, u h_{3}\right)$ for any scalar $u$ and any ordered triple $\boldsymbol{h}=\left(h_{1}, h_{2}, h_{3}\right)$ in $\mathbb{A}_{d}$. It is clear that the curves $\mathcal{C}_{\boldsymbol{g}}$ and $\mathcal{C}_{\sigma \cdot \boldsymbol{g}}$ have the same configuration of singularities; and therefore, $\Sigma \cdot S_{\%}=S_{\%}$ for all $\%$ from $(0.3)$.

Fix $\%$. We have seen that the set $S_{\%}$ is non-empty. Fix $\boldsymbol{g} \in S_{\%}$ and define

$$
\theta_{\boldsymbol{g}}: \Sigma \rightarrow S_{\%}
$$

by $\theta_{\boldsymbol{g}}(\sigma)=\sigma \cdot \boldsymbol{g}$. Let $\overline{\operatorname{im} \theta_{\boldsymbol{g}}}$ and $\overline{S_{\%}}$ be the closure of $\operatorname{im} \theta_{\boldsymbol{g}}$ and $S_{\%}$, respectively, in $\mathbb{T}_{d}$. The group $\Sigma$ is an irreducible closed algebraic variety of dimension 12 . It follows that $\operatorname{im} \theta_{\boldsymbol{g}}$; and therefore, $\overline{\operatorname{im} \theta_{\boldsymbol{g}}}$ are irreducible subsets of $\mathbb{T}_{d}$. Thus, $\theta_{\boldsymbol{g}}: \Sigma \rightarrow \overline{\operatorname{im} \theta_{\boldsymbol{g}}}$ is a dominate morphism of closed irreducible affine algebraic varieties over an algebraically closed field. A quick calculation shows that if $\boldsymbol{h}$ is in $\operatorname{im} \theta_{\boldsymbol{g}}$, then the fiber $\theta_{\boldsymbol{g}}^{-1}(\boldsymbol{h})$ of $\theta_{\boldsymbol{g}}$ over $\boldsymbol{h}$ is a finite set of points; so $\operatorname{dim} \theta_{\boldsymbol{g}}^{-1}(\boldsymbol{h})=0$. The Fiber Dimension Theorem applies, exactly as it did in (6.15) and (6.16), to yield that

$$
\operatorname{dim}\left(\overline{\operatorname{im} \theta_{\boldsymbol{g}}}\right)=\operatorname{dim}(\Sigma)-\operatorname{dim}(\text { a generic fiber })=12-0=12 .
$$

We have $\overline{\operatorname{im} \theta_{\boldsymbol{g}}}$ is a closed irreducible subset of $\overline{S_{\%}}$ of dimension 12 . We conclude that $\operatorname{dim}\left(S_{\%}\right)=\operatorname{dim}\left(\overline{S_{\%}}\right) \geq \operatorname{dim}\left(\overline{\operatorname{im} \theta_{\boldsymbol{g}}}\right)=12$.

Remark 9.15. The proof of Observation 9.13 shows that if $S$ is any non-empty subset of $\mathbb{T}_{d}$ which is closed under the action of $\Sigma$, and $\bar{S}$ is the closure of $S$ in $\mathbb{T}_{d}$, then every irreducible component of $\bar{S}$ has dimension at least 12 . 
Proposition 9.16. The statements about dimension in Theorems 9.10 and 9.12 are correct.

Proof. Consider

$$
S_{(3: 1)}=T_{(3: 1)}^{\mathrm{QCP}} \subsetneq T_{(3: 1,1)}^{\mathrm{QCP}} \subsetneq T_{(3: 1,1,1)}^{\mathrm{QCP}} \subsetneq T_{(2: 1,1)^{3}}^{\mathrm{QCP}}
$$

Each set is irreducible by Proposition 9.7 and closed in $\mathbb{T}_{4}$ by Proposition 9.3 . One has

$$
12 \leq \operatorname{dim} T_{(3: 1)}^{\mathrm{QCP}}<\operatorname{dim} T_{(3: 1,1)}^{\mathrm{QCP}}<\operatorname{dim} T_{(3: 1,1,1)}^{\mathrm{QCP}}<\operatorname{dim} T_{(2: 1,1)^{3}}^{\mathrm{QCP}}=15 .
$$

One concludes that

$$
\operatorname{dim} T_{(3: 1)}^{\mathrm{QCP}}=12, \quad \operatorname{dim} T_{(3: 1,1)}^{\mathrm{QCP}}=13, \quad \text { and } \quad \operatorname{dim} T_{(3: 1,1,1)}^{\mathrm{QCP}}=14
$$

Consider

$$
S_{(2: 2: 2: 1)}=T_{(2: 2: 2: 1)}^{\mathrm{BQP}} \subsetneq T_{(2: 2: 2: 1,1)}^{\mathrm{BQP}} \subsetneq T_{(2: 2: 1,1),(2: 1,1)}^{\mathrm{BQP}} \subsetneq T_{(2: 1,1)^{3}}^{\mathrm{BQP}}=\mathbb{B}_{4} .
$$

Each set is irreducible by Proposition 9.7 and the fact that $\mathbb{B}_{4}$ is an open subset of the irreducible space $\mathbb{A}_{4}$; hence, $\mathbb{B}_{4}$ is also an irreducible space. Each set is closed in $\mathbb{B}_{4}$ by Proposition 9.5. One has

$$
12 \leq \operatorname{dim}\left(T_{(2: 2: 2: 1)}^{\mathrm{BQP}}\right)<\operatorname{dim}\left(T_{(2: 2: 2: 1,1)}^{\mathrm{BQP}}\right)<\operatorname{dim}\left(T_{(2: 2: 1,1),(2: 1,1)}^{\mathrm{BQP}}\right)<\operatorname{dim} \mathbb{B}_{4}=15 ;
$$

and therefore,

$$
\operatorname{dim}\left(T_{(2: 2: 2: 1)}^{\mathrm{BQP}}\right)=12, \operatorname{dim}\left(T_{(2: 2: 2: 1,1)}^{\mathrm{BQP}}\right)=13, \quad \operatorname{dim}\left(T_{(2: 2: 1,1),(2: 1,1)}^{\mathrm{BQP}}\right)=14
$$

Consider

$$
T_{(2: 1)^{3}}^{\mathrm{BQP}} \subsetneq T_{(2: 1,1),(2: 1)^{2}}^{\mathrm{BQP}} \subsetneq T_{(2: 1,1)^{2},(2: 1)}^{\mathrm{BQP}} \subseteq T_{(2: 1,1)^{3}}^{\mathrm{BQP}}=\mathbb{B}_{4} .
$$

All four sets are closed in $\mathbb{B}_{4}$ and irreducible. It follows that

$$
12 \leq \operatorname{dim} T_{(2: 1)^{3}}^{\mathrm{BQP}}<\operatorname{dim}\left(T_{(2: 1,1),(2: 1)^{2}}^{\mathrm{BQP}}\right)<\operatorname{dim}\left(T_{(2: 1,1)^{2},(2: 1)}^{\mathrm{BQP}}\right)<\operatorname{dim} \mathbb{B}_{4}=15
$$

and

$$
\operatorname{dim} T_{(2: 1)^{3}}^{\mathrm{BQP}}=12, \quad \operatorname{dim}\left(T_{(2: 1,1),(2: 1)^{2}}^{\mathrm{BQP}}\right)=13, \quad \operatorname{dim}\left(T_{(2: 1,1)^{2},(2: 1)}^{\mathrm{BQP}}\right)=14
$$


One may also consider the following chain of closed irreducible subsets of $\mathbb{T}_{4}$ :

$$
T_{(3: 1,1)}^{\mathrm{QCP}} \subsetneq T_{(2,1,1)^{2},(2: 1)}^{\mathrm{QCP}} \subsetneq T_{(2: 1,1)^{3}}^{\mathrm{QCP}}
$$

to conclude $\operatorname{dim} T_{(2,1,1)^{2},(2: 1)}^{\mathrm{QCP}}=14$.

Consider

$$
S_{(2: 2: 1),(2: 1)}=T_{(2: 2: 1),(2: 1)}^{\mathrm{BQP}} \subsetneq T_{(2: 2: 1),(2: 1,1)}^{\mathrm{BQP}}, T_{(2: 2: 1,1),(2: 1)}^{\mathrm{BQP}} \subsetneq T_{(2: 2: 1,1),(2: 1,1)}^{\mathrm{BQP}} .
$$

All four sets are closed in $\mathbb{B}_{4}$ and irreducible by Proposition 9.7. The dimension of the set on the right was calculated in (9.17). We have

$$
12 \leq \operatorname{dim} T_{(2: 2: 1),(2: 1)}^{\mathrm{BQP}}<\operatorname{dim} T_{(2: 2: 1),(2: 1,1)}^{\mathrm{BQP}}, \operatorname{dim} T_{(2: 2: 1,1),(2: 1)}^{\mathrm{BQP}}<\operatorname{dim} T_{\substack{(2: 2: 1,1),(2: 1,1)}}^{\mathrm{BQP}}=14
$$

and we conclude that

$$
\operatorname{dim} T_{(2: 2: 1),(2: 1)}^{\mathrm{BQP}}=12 \quad \text { and } \quad \operatorname{dim} T_{(2: 2: 1),(2: 1,1)}^{\mathrm{BQP}}=T_{(2: 2: 1,1),(2: 1)}^{\mathrm{BQP}}=13
$$

Proposition 9.18. The sets $S_{(2: 2: 2: 1)}$ and $S_{(2: 2: 1),(2: 1)}$ are closed in $\mathbb{T}_{4}$.

Proof. Let \% equal $(2: 2: 2: 1)$ or $(2: 2: 1),(2: 1)$. We saw in Proposition 9.16 that $\operatorname{dim} S_{\%}=12$. Let $\bar{S}_{\%}$ be the closure of $S_{\%}$ in $\mathbb{T}_{4}$. We saw in Proposition 9.3 that $T_{\%}^{Q C P}$ is a closed subset of $\mathbb{T}_{4}$. It follows that $\overline{S_{\%}} \subseteq T_{\%}^{Q C P}$. On the other hand, we know from Table 2 that $T_{\%}^{Q C P} \cap \mathbb{B}_{4}=S_{\%}$. To prove this result we must show that $\overline{S_{\%}} \cap \mathbb{U B}_{4}=\emptyset$. (Recall from Definition 5.4 that $\mathbb{U B}_{4}$ is the closed subset $\mathbb{T}_{4} \backslash \mathbb{B}_{4}$ of $\mathbb{T}_{4}$.) Suppose that $\overline{S_{\%}} \cap \mathbb{U B}_{4}$ is non-empty. It is clear that $\overline{S_{\%}} \cap \mathbb{U B}_{4}$ is closed under the action of the group $\Sigma$. Let $X$ be a closed irreducible component of $\overline{S_{\%}} \cap \mathbb{U B}_{4}$. It follows from Remark 9.15 that $\operatorname{dim} X \geq 12$. It is not possible to have two closed irreducible sets $X \subsetneq \overline{S_{\%}}$ with $\operatorname{dim} X \geq 12$ and $\operatorname{dim} \overline{S_{\%}}=12$.

Acknowledgment. Critical conversations which lead to this work occurred when the authors were all in the same city. The authors are especially appreciative of the travel support which brought them together at the Pan-American Advanced Study Institute (PASI) conference on Commutative Algebra and its Connections to Geometry, in honor of Wolmer Vasconcelos, in Olinda, Brazil in August, 2009 and the Midwest Algebra, Geometry and their Interactions Conference (MAGIC'10) in Notre Dame, Indiana in April, 2010. 


\section{REFERENCES}

1. S. Abhyankar, Algebraic Geometry for Scientists and Engineers, Mathematical Surveys and Monographs 35, American Mathematical Society, Providence, RI, 1990.

2. A. B. Basset, An Elementary Treatise on Cubic and Quartic Curves, Deighton Bell, London, 1901.

3. J. W. Bruce and P. J. Giblin, A stratification of the space of plane quartic curves, Proc. London Math. Soc. (3) 42 (1981), 270-298.

4. W. Bruns and J. Herzog, Cohen-Macaulay Rings, Cambridge Studies in Advanced Mathematics 39, Cambridge University Press, Cambridge, 1993.

5. W. Bruns and U. Vetter, Determinantal Rings, Lecture Notes in Mathematics 1327, SpringerVerlag, Berlin, 1988.

6. D. Buchsbaum and D. Eisenbud, What makes a complex exact?, J. Algebra 25 (1973), 259268.

7. L. Busé, On the equations of the moving curve ideal of a rational algebraic plane curve, J. Algebra 321 (2009), 2317-2344.

8. L. Busé and T. Luu Ba, Matrix-based implicit representations of rational algebraic curves and applications, Comp. Aided Geom. Design 27 (2010), 681-699.

9. L. Busé and C. D'Andrea, Singular factors of rational plane curves, preprint (2009), available on the arXiv.

10. F. Chen, W. Wang, and Y. Liu, Computing singular points of plane rational curves, J. Symbolic Comput. 43 (2008), 92-117.

11. D. Eisenbud, Commutative Algebra: with a View Toward Algebraic Geometry, Graduate Texts in Mathematics 150, Springer-Verlag, New York, 1995.

12. D. Eisenbud, The Geometry of Syzygies, A second course in Commutative Algebra and Algebraic Geometry, Springer-Verlag, New York, 2005.

13. D. Eisenbud, C. Huneke, and B. Ulrich, A simple proof of some generalized principal ideal theorems, Proc. Amer. Math. Soc. 129 (2001), 2535-2540.

14. D. Eisenbud and B. Ulrich, Row ideals and fibers of morphisms, Michigan Math. J. 57 (2008), 261-268.

15. M. Green, The Eisenbud-Koh-Stillman conjecture on linear syzygies, Invent. Math. 136 (1999), 411-418.

16. R. Hartshorne, Algebraic Geometry, Graduate Texts in Mathematics 52, Springer-Verlag, New York, 1977.

17. H. Hilton, Plane Algebraic Curves, Clarendon Press, Oxford, 1920.

18. M. Hochster, Properties of Noetherian rings stable under general grade reduction, Arch. Math. (Basel) 24 (1973), 393-396.

19. M. Hochster, Topics in the Homological Theory of Modules over Commutative Rings, Conference Board of the Mathematical Sciences Regional Conference Series in Mathematics 24, American Mathematical Society, Providence, R.I., 1975.

20. C. Huneke and M. Miller, A note on the multiplicity of Cohen-Macaulay algebras with pure resolutions, Canad. J. Math. 37 (1985), 1149-1162.

21. D. Katz, On the number of minimal prime ideals in the completion of a local domain, Rocky Mountain J. Math. 16 (1986), 575-578.

22. E. Kunz, Kähler Differentials, Advanced Lectures in Mathematics, Friedr. Vieweg and Sohn, Braunschweig, 1986.

23. E. Kunz, Introduction to Plane Algebraic Curves, Birkhäuser Boston, Inc., Boston, MA, 2005.

24. A. Kustin, C. Polini, and B. Ulrich, Defining equations of Rees algebras, (in preparation). 
25. A. Kustin, C. Polini, and B. Ulrich, Blowups and fibers of morphisms, (in preparation).

26. H. Matsumura, Commutative Ring Theory, Second edition, Cambridge Studies in Advanced Mathematics 8, Cambridge University Press, Cambridge, 1989.

27. H. Matsumura, Commutative Algebra, Second edition, Mathematics Lecture Note Series 56, Benjamin/Cummings Publishing Co., Inc., Reading, MA, 1980.

28. M. Nagata, Local Rings, Interscience Tracts in Pure and Applied Mathematics 13, John Wiley \& Sons, New York-London, 1962.

29. M. Namba, Geometry of Projective Algebraic Curves, Dekker, New York, 1984.

30. N. Song, F. Chen, and R. Goldman, Axial moving lines and singularities of rational planar curves, Comput. Aided Geom. Design 24 (2007), 200-209.

31. J. Sendra, F. Winkler, and S. Pérez-Díaz, Rational Algebraic Curves. A Computer Algebra Approach, Algorithms and Computation in Mathematics 22, Springer-Verlag, Berlin, 2008.

32. I. Swanson and C. Huneke, Integral Closure of Ideals, Rings, and Modules, London Mathematical Society Lecture Note Series 336, Cambridge University Press, Cambridge, 2006.

33. C. T. C. Wall, Singular Points of Plane Curves, London Mathematical Society Student Texts 63, Cambridge University Press, Cambridge, 2004.

34. C. T. C. Wall, Geometry of quartic curves, Math. Proc. Cambridge Philos. Soc. 117 (1995), 415-423.

35. O. Zariski and P. Samuel, Commutative Algebra. Vol. II, Graduate Texts in Mathematics 29, Springer-Verlag, New York, 1975.

Department of Mathematics, Amherst College, Amherst, MA 01002-5000

E-mail address: dac@math.amherst.edu

Mathematics Department, University of South Carolina, Columbia, SC 29208

E-mail address: kustin@math.sc.edu

Mathematics Department, University of Notre Dame, Notre Dame, IN 46556

E-mail address: cpolini@nd.edu

Department of Mathematics, Purdue University, West Lafayette, IN 47907

E-mail address: ulrich@math.purdue.edu 